Imperial College London

Department of Computing

\title{
Computationally Unifying Urban Masterplanning
}

\author{
David Alan Birch
}

Spring 2013

\author{
Supervised by \\ Paul Kelly, Tony Field \\ Industrial Supervisor: \\ Alvise Simondetti
}

Submitted in fulfilment of the requirements for the degree of Doctor of Philosophy in Computing of Imperial College London and the Diploma of Imperial College London 



\section{Declaration}

The material presented in this thesis is the author's own, except where it appears with attribution to others.

This work has been undertaken under an EPSRC CASE award grant and sponsorship from Arup.

Chapter 4 (p139) is a collaborative case study with Arup North America. Application of the HierSynth framework was undertaken by the author. Design data and analysis models were provided by Arup. Investigations were set up and run by the author but designed and analysed collaboratively with Arup engineers. The author gratefully acknowledges the support and insight of the Arup team involved in this project.

Chapter 5 (p209) is joint work with Helen Liang of Bath University. The tool suite was created by the author from discussion with Helen Liang. Analysis of IRM Concept and 2009 models was collaborative, analysis of IRM 2011 model was undertaken by the author.

The copyright of this thesis rests with the author and is made available under a Creative Commons Attribution Non-Commercial No Derivatives licence. Researchers are free to copy, distribute or transmit the thesis on the condition that they attribute it, that they do not use it for commercial purposes and that they do not alter, transform or build upon it. For any reuse or redistribution, researchers must make clear to others the licence terms of this work.

David Alan Birch 



\section{Abstract}

Urban masterplanning is the process of creating a coherent design for developing a campus, suburb, city or region.

Unfortunately these design and analysis teams face challenges which prevent rapid quantitative analysis of design iterations; precluding potential design improvement. These include limited automation, poor integration of modelling disciplines and, in particular, very limited scope for design space exploration.

This thesis investigates these challenges and their solutions. A computational framework HierSynth is presented to help computationally unify the design and analysis sides of the urban masterplanning community.

The key contribution of this thesis is HierSynths data model. This presents a reconceptualization of the workflow graph by composing it with tree based designdecompositions commonly found in architectural interoperability formats. This is achieved through a hierarchy of design queries, templates and analyses which when executed form a design hierarchy annotated with evaluated analyses. This enables detailed multi-scale analysis directly on design elements whilst supporting scenario generation and design space exploration capabilities and techniques to explore design improvements.

The HierSynth framework is evaluated by application to a major commercial masterplanning project with Arup North America and is used to explore the most effective techniques for generating design insight. HierSynth enabled an order-of-magnitude more analysis iterations and previously infeasible design space exploration to answer design questions.

During this collaboration an unexpected challenge was identified in maintaining and debugging complex, highly interrelated analysis models implemented as spreadsheets. A toolkit to address this is developed and applied to several generations of complex multi-disciplinary sustainability models.

In summary this thesis presents evidence of the need for, implementation of, and practical benefits from, computationally unifying urban masterplanning design and analysis. The key contribution is a compositional data model supporting this unification. Finally avenues for further work are explored to further aid this community including data provenance and supporting smart cities. 



\section{Imperial College}

London

David Birch,

Paul Kelly,

Tony Field Unifying Urban Masterplan

Dept. Computing
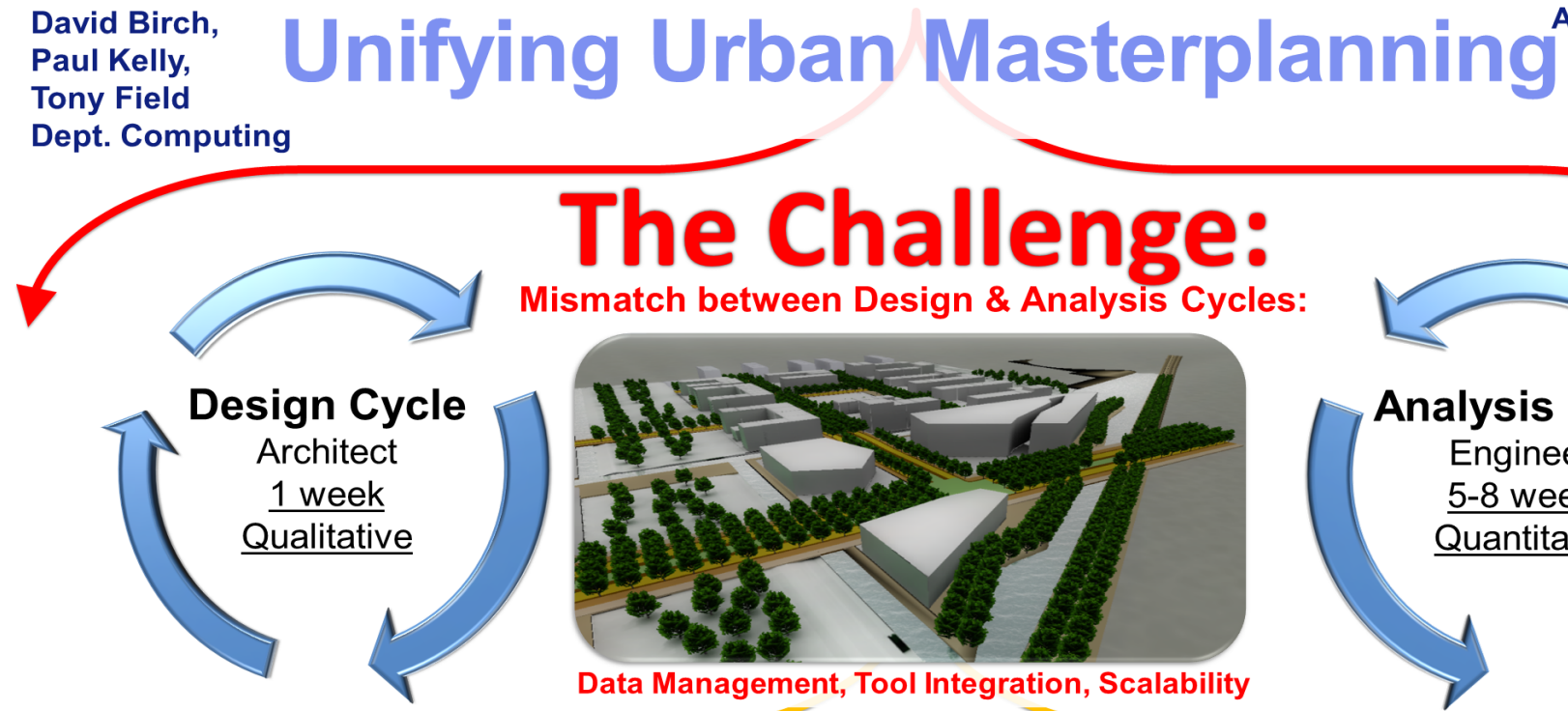

Alvise Simondetti CommunityB EPSRC
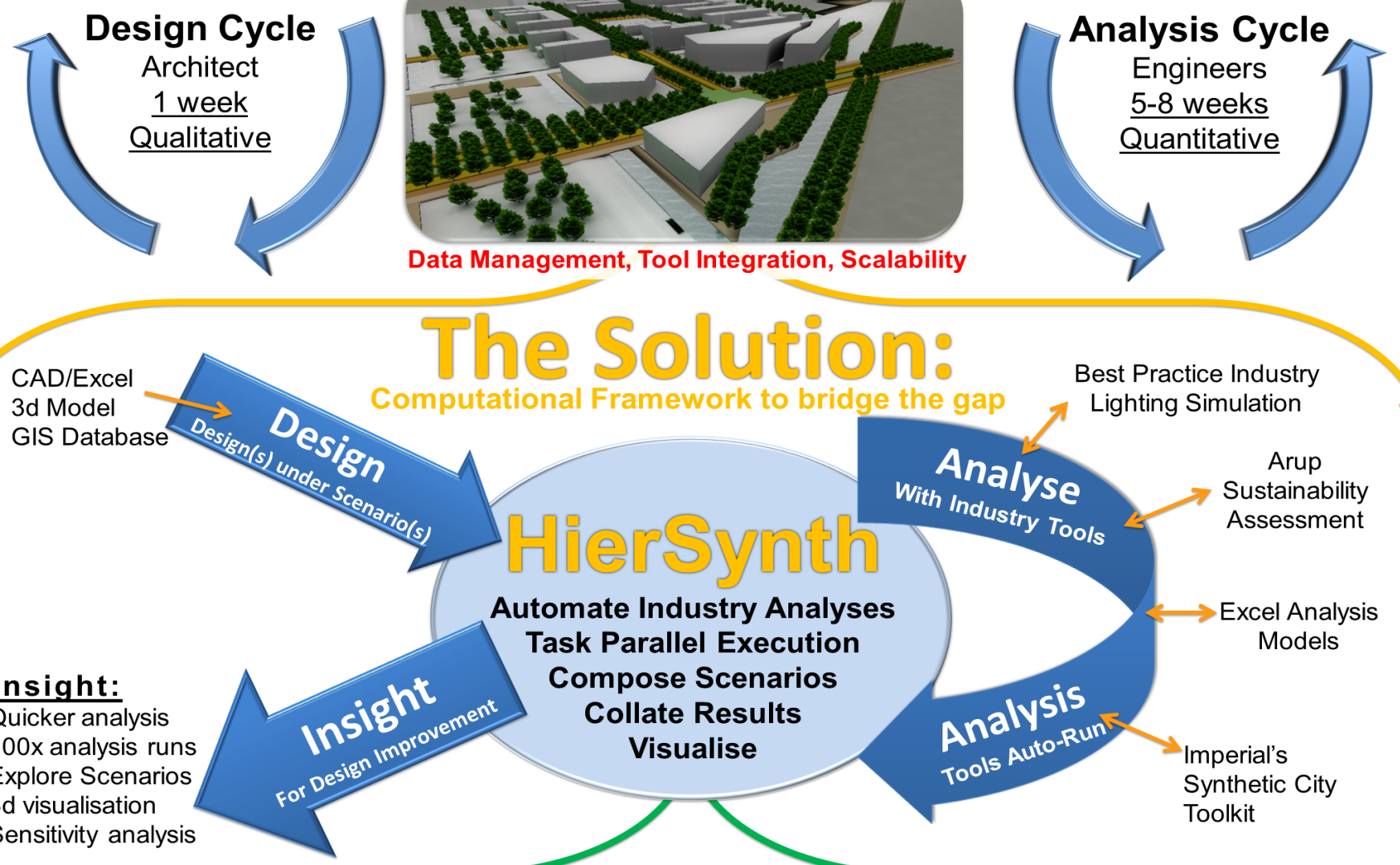

Automate Industry Analyses Task Parallel Execution

\section{Detailed Insight: 200x more data shows} trends \& anomalies

\section{Geo-located Per Capita Carbon}

3 Designs \& 3 Energy Scenarios

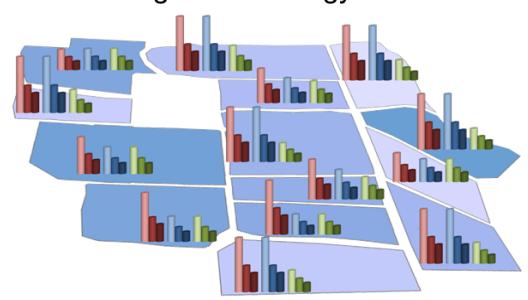

Per Capita Carbon

Per District \& Construction Phase

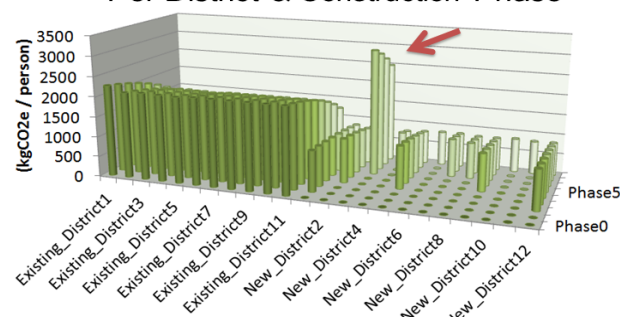

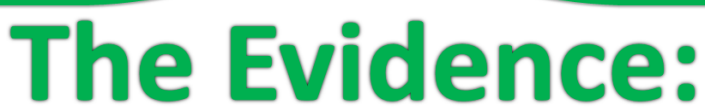

Applying HierSynth to live project with Arup North America working as part of team, analyzing 3 masterplans under 3 energy scenarios using 3 integrating models for:

1. Sustainability Assessment

2. District Energy System Feasibility

3. Life Cycle Costing of District Energy

\section{Integrated 3d Visualisation}

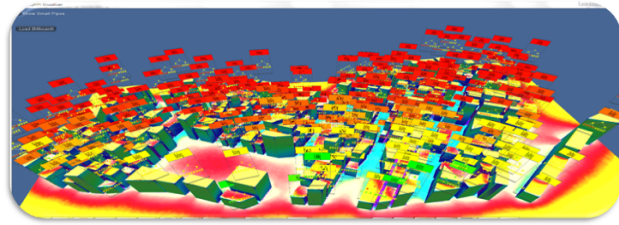

"Overall HierSynth has quickly generated results in lists and charts that allow evaluation of results across a number of models and districts that would not be possible to generate in the time allowed for the project, otherwise." Arup Engineer

Buildings Sustainability Group
Best Practice Industry Lighting Simulation 



\section{Dedication}

I would like to thank the following people, without whom this thesis would not have been possible.

- My academic supervisors Paul Kelly and Tony Field - For your support, experience, wisdom and willingness to undertake a project outside of the norm. It has been a honour to be under your supervision.

- My industrial supervisor Alvise Simondetti - For your endless enthusiasm and insight into the world of architectural design and analysis; it has been a pleasure to learn from and with you.

- My examiners Steve Easterbrook and Duncan Gillies - For your insight into and fascinating discussion of this thesis without which it would be markedly poorer.

- Helen Liang - For your enthusiasm in showing and solving the challenges of spreadsheet modelling. I am glad to have collaborated with you.

- The "Community B" team - For being willing to take a risk on using the HierSynth framework. Your insight, professionalism and endless support made our collaboration a success. It was a pleasure to work with you.

- Arup - To all of the practitioners I have met; your experiences, comments and feedback have helped to make this thesis what it is. Particularly the FII group for their advice and welcome.

- The Software Performance Optimisation Group - For making life in the lab far more interesting.

- All at Christ Church Mayfair - For your friendship and encouragement in the truth through the years.

- My housemates over the years at Lillie road - For your encouragement, banter and for enduring endless pranks. It was an honour to share life with all twenty-five of you during seven years of studies at Imperial.

- My family - for their ceaseless support and encouragement.

"My flesh and my heart may fail,

but God is the strength of my heart

and my portion forever."

Psalm 73v26

\section{Soli Deo Gloria}





\section{Contents}

1 Introduction $\quad 29$

1.1 Contributions . . . . . . . . . . . . . . . . . . . 32

1.2 Structure of Thesis . . . . . . . . . . . . . . . 33

1.3 Publications . . . . . . . . . . . . . . . . 33

1.3.1 HierSynth . . . . . . . . . . . . . . . . . . 33

1.3 .2 Spreadsheet Analysis . . . . . . . . . . . . . . . . . . . . . . . . . . . . . . . . . . 35

1.3 .3 Industry Vision . . . . . . . . . . . . . . . . . . . . 35

2 Techniques for Computationally Unifying Urban Masterplanning $\quad 37$

2.1 Research Fronts . . . . . . . . . . . . . . . . . 37

2.2 Architectural Industry Challenges . . . . . . . . . . . . . . . . . . . 39

2.3 Computational Research Challenges . . . . . . . . . . . . . . 47

2.3 .1 Focus of this Thesis . . . . . . . . . . . . 50

2.4 Product Optimisation Frameworks ．.. . . . . . . . . . . . 51

2.4 .1 Comsol Multiphysics . . . . . . . . . . . . . . . 51

2.4.2 Model Center . . . . . . . . . . . . . . . . . . . . . 52

2.4 .3 ModeFrontier . . . . . . . . . . . . . . . . . . . 53

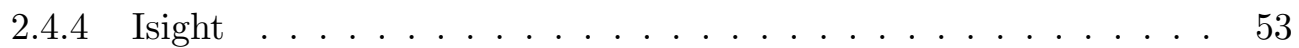

2.4 .5 Conclusions . . . . . . . . . . . . . . . . . 54

2.5 Architectural Optimisation Frameworks . . . . . . . . . . . 56

2.5.1 Intelligent Design Objects . . . . . . . . . . . . . 56

2.5.2 Multidisciplinary Process Integration and Design Optimization . 58

2.5.3 Component-oriented Decomposition for Multidisciplinary Design

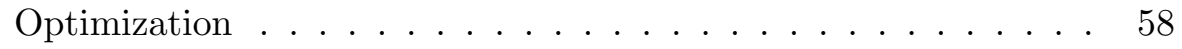

2.5.4 Conclusions . . . . . . . . . . . . . . . . . . 60

2.6 Integrated Design Analysis Packages . . . . . . . . . . . . . . . . . 62

2.6.1 Autodesk Suite . . . . . . . . . . . . . . . . . . . 62

2.6 .2 CityCad . . . . . . . . . . . . . . . . . 64

2.6 .3 Conclusions . . . . . . . . . . . . . . . . 65

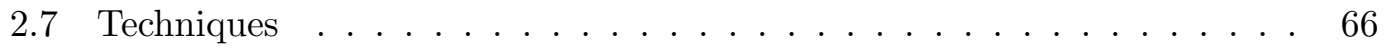

2.7 .1 Visualisation . . . . . . . . . . . . . 66

2.7 .2 Sensitivity Analysis _. . . . . . . . . . . . . 68 
2.7 .3 Performance Trees . . . . . . . . . . . . . . . . . . . . . . 69

2.8 Integration Systems \& Formats . . . . . . . . . . . . . 71

2.8 .1 Collada . . . . . . . . . . . . . . . . . 72

2.8 .2 CityGML . . . . . . . . . . . . . . . . 72

2.8 .3 Green Building XML . . . . . . . . . . . . . . . . . 73

2.8 .4 BIM . . . . . . . . . . . . . . . . . 73

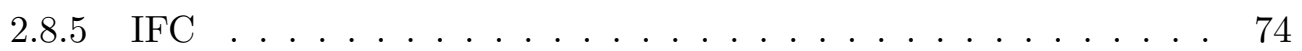

2.8 .6 DesignLink SDK . . . . . . . . . . . . . . 75

2.8.7 Geographic Information Systems . . . . . . . . . . . . 75

2.8.8 Feature Manipulation Engine (FME) . . . . . . . . . . . . 76

2.8 .9 Conclusions . . . . . . . . . . . . . . . . . 76

2.9 Conclusions . . . . . . . . . . . . . . . . . . . 77

2.10 Hypotheses for computationally unifying urban masterplanning . . . . . 80

2.10 .1 Approaches . . . . . . . . . . . . . . . . 80

2.10 .2 Techniques . . . . . . . . . . . . . . . 81

3 HierSynth $\quad \mathbf{8 5}$

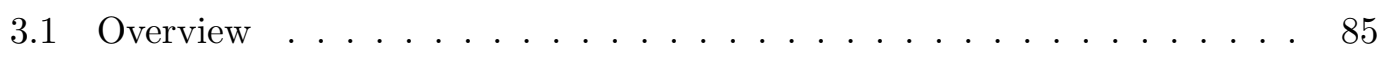

3.2 HierSynth . . . . . . . . . . . . . . . . . . . 85

3.3 HierSynth Design Goals . . . . . . . . . . . . . . . . . . . 86

3.4 HierSynth Data Model Executive Summary . . . . . . . . . . . . . . . 88

3.4 .1 HierSynth Tree . . . . . . . . . . . . . . . . . . . . 89

$3.4 .2 \quad$ Execution Chains . . . . . . . . . . . . . . . . . . . . . 91

3.4.3 Core Concepts . . . . . . . . . . . . . . . . . . . . . 92

3.5 Advantages and Disadvantages . . . . . . . . . . . . . . . . 94

3.5 .1 Advantages . . . . . . . . . . . . . . . . . . 94

3.5 .2 Disadvantages . . . . . . . . . . . . . . . . . . . 95

$3.6 \quad$ Full Data Model . . . . . . . . . . . . . . . . . . . . . . . . . . 96

3.6.1 Data \& Scenarios . . . . . . . . . . . . . . . . 96

$3.6 .2 \quad$ Expression Language . . . . . . . . . . . . . . . . . . 97

3.6 .3 Tree structure . . . . . . . . . . . . . . . . . . . 98

3.6.4 Node Types . . . . . . . . . . . . . . . . . . . . . . . . . 98

3.6.5 Data flow . . . . . . . . . . . . . . . . . 99

3.6.6 Analysis Requirements . . . . . . . . . . . . . . . . . . 100

3.7 Tree Execution . . . . . . . . . . . . . . . . . . . . . 100

3.7.1 Expand Phase . . . . . . . . . . . . . . . . . . . 102

3.7.2 Execute Phase . . . . . . . . . . . . . . . . 105

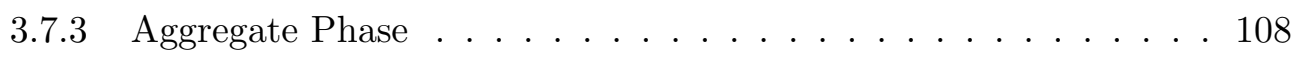

3.8 Performance Trees . . . . . . . . . . . . . . . . . . . 108 
3.9 Graph Structure . . . . . . . . . . . . . . . . . . . . . 111

3.9 .1 Execution Links . . . . . . . . . . . . . . . . . . 111

3.9.2 SynCity Analysis Example . . . . . . . . . . . . . . . 112

3.10 Execution Chains . . . . . . . . . . . . . . . . . . . . . . 114

3.11 Scenario Generation . . . . . . . . . . . . . . . . 120

3.12 Performance . . . . . . . . . . . . . . . . . . . . . 120

3.12.1 Parallelisation . . . . . . . . . . . . . . . . . . . 122

3.12 .2 Application Sharing . . . . . . . . . . . . . . . . . . 124

3.12 .3 Caching . . . . . . . . . . . . . . . . . 125

3.13 Implementation . . . . . . . . . . . . . . . . . . . 125

3.13 .1 Plugins . . . . . . . . . . . . . . . . 126

3.14 Sensitivity Analysis . . . . . . . . . . . . . . . . . 127

3.14 .1 Possible Extensions . . . . . . . . . . . . . . . . . . 132

3.15 Visualisation . . . . . . . . . . . . . . . . . . . 132

3.16 Workflow . . . . . . . . . . . . . . . . . 133

3.17 HierSynth in Context . . . . . . . . . . . . . . . . . . . . . 134

3.17 .1 Geographic Information Systems . . . . . . . . . . . . 134

3.17.2 Computational Design Optimisation (CDO) . . . . . . . . 134

3.17 .3 Interoperability Standards . . . . . . . . . . . . . . 135

3.17.4 Design / Analysis Suites . . . . . . . . . . . . . . . 135

3.17 .5 Building Scale . . . . . . . . . . . . . . . 136

3.18 Conclusions . . . . . . . . . . . . . . . . 136

4 Case Study - CommunityB 139

4.1 Introduction . . . . . . . . . . . . . . . . . . . . . . 139

4.2 Research Methodology . . . . . . . . . . . . . . . . . . . . . . 141

4.3 Case Study Research Questions . . . . . . . . . . . . . . . . . . . 141

4.4 Models . . . . . . . . . . . . . . . . . . . . . . . . . . . . . . 144

4.4 .1 Radiance ... . . . . . . . . . . . . . . . 144

4.4.2 Integrated Resource Management . . . . . . . . . . . . . . . . . . . . . . . . . . . . . . . . . . .

4.4 .3 District Energy Feasibility . . . . . . . . . . . . . . . . . . 145

4.4.4 Life-cycle Costing . . . . . . . . . . . . . . . . 146

4.5 Model Automation . . . . . . . . . . . . . . . . . . . 146

4.6 Scenario Generation ． . . . . . . . . . . . . . . . . . . . . 148

4.7 Automated Results . . . . . . . . . . . . . . . . . . 151

4.8 Analysis over Time . . . . . . . . . . . . . . . . . . . . . . . . . . . . . . . . . . . . . . . . . . . . . . .

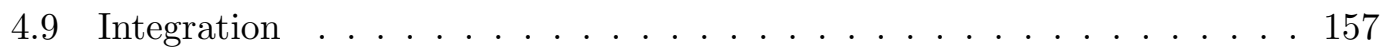

4.9 .1 Implementation . . . . . . . . . . . . . . . . . . 159

4.9 .2 Challenges . . . . . . . . . . . . . . . 161 
4.10 Integrated Results . . . . . . . . . . . . . . . . . . . 162

4.11 Block-level Analysis . . . . . . . . . . . . . . . . . . 166

4.11 .1 Lighting Analysis . . . . . . . . . . . . . . . . . 166

4.11 .2 Block Performance . . . . . . . . . . . . . . . 168

4.11 .3 Further Work . . . . . . . . . . . . . . . . . . . . . 171

4.12 Insight . . . . . . . . . . . . . . . . . . . . . 171

4.12 .1 Effect of Electric Vehicles . . . . . . . . . . . . . . . 172

4.12.2 Optimal Density for District Energy System . . . . . . . . . . . 175

4.12 .3 Residential and Commercial Mix . . . . . . . . . . . . 177

4.13 Sensitivity Analysis . . . . . . . . . . . . . . . . . . . . . 187

4.13 .1 IRM analysis . . . . . . . . . . . . . . . . 187

4.13 .2 Strategy Analysis . . . . . . . . . . . . . . . . . . 190

4.14 Collaboration Conclusions . . . . . . . . . . . . . . . . . . 196

4.14 .1 Top Five Capabilities ． . . . . . . . . . . . . . . . . . . 198

4.14 .2 Top Five Findings . . . . . . . . . . . . . . . . . . . . 198

4.15 Framework Evaluation . . . . . . . . . . . . . . . . . . . . 199

4.15 .1 What Surprised us . . . . . . . . . . . . . . 199

4.15 .2 What Worked . . . . . . . . . . . . . . . . . . 201

4.15 .3 What Didn't . . . . . . . . . . . . . . . . 202

4.15 .4 Additional Computational Needs . . . . . . . . . . . . . . 203

4.15.5 Areas for Further Investigation . . . . . . . . . . . . . 205

5 Extraction and Analysis Methodology 209

5.1 Introduction . . . . . . . . . . . . . . . . . . . . . 209

5.2 Motivation . . . . . . . . . . . . . . . . . 211

5.3 Methodology . . . . . . . . . . . . . . . . . . . 215

5.4 Related Work . . . . . . . . . . . . . . . . . . . . . . 215

5.5 Model Extraction . . . . . . . . . . . . . . . . . . . . . . 216

5.6 Visualisation . . . . . . . . . . . . . . . . . 217

5.6 .1 Visualisation Case Study . . . . . . . . . . . . . . . . 218

5.7 Model Metrics . . . . . . . . . . . . . . . . . . . . . 219

5.8 Discipline Coupling . . . . . . . . . . . . . . . . . . . . . . 221

5.8 .1 Software Metrics . . . . . . . . . . . . . . . . 222

5.9 Formula Metrics . . . . . . . . . . . . . . . . . . . . . . . 224

5.9 .1 Formula Length . . . . . . . . . . . . . . . . . . 225

5.9 .2 Hard Coded Constants . . . . . . . . . . . . . . . . . . 225

5.9 .3 Common Sub-expressions . . . . . . . . . . . . . . 226

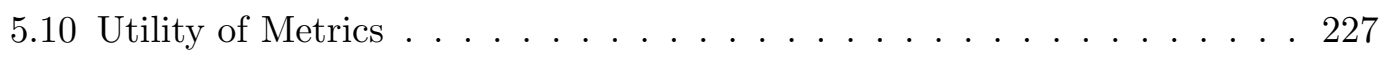

5.10 .1 Aiding Comprehension . . . . . . . . . . . . . . . 227 
5.10 .2 Improving Quality . . . . . . . . . . . . . . . . . . 228

5.10 .3 Guiding Development . . . . . . . . . . . . . . . 228

5.10 .4 Conclusions . . . . . . . . . . . . . . . . . . . . . 229

5.11 Sensitivity Analysis . . . . . . . . . . . . . . . . . . . . . 229

5.11 .1 Case Study . . . . . . . . . . . . . . . . . . 230

5.12 IRM Evolution . . . . . . . . . . . . . . . . . . . . 232

5.13 Further Work . . . . . . . . . . . . . . . . . . 235

5.14 Conclusions . . . . . . . . . . . . . . . . . . 235

$\begin{array}{llr}6 & \text { Conclusions } & 237\end{array}$

6.1 Executive Summary . . . . . . . . . . . . . . . . . 237

6.2 Critical Analysis . . . . . . . . . . . . . . . . . . . . . 240

6.2 .1 Approach . . . . . . . . . . . . . . . . . . 240

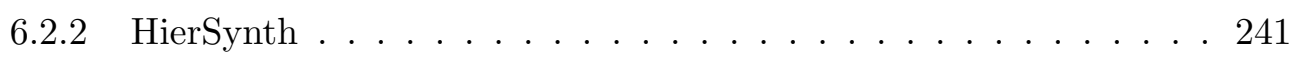

6.2 .3 Data Model . . . . . . . . . . . . . . . . . . . . . . . . 242

6.2 .4 Case Study . . . . . . . . . . . . . . . . . . . . 243

6.2 .5 Engineering Assessment . . . . . . . . . . . . . . . 243

6.2 .6 Designer Interaction . . . . . . . . . . . . . . . . . 244

6.2.7 Computational Design Optimisation . . . . . . . . . . . . 244

6.3 Thesis Conclusions . . . . . . . . . . . . . . . . . . . . 245

6.4 Contributions . . . . . . . . . . . . . . . . . . 247

6.5 Further Work . . . . . . . . . . . . . . . . . . . . . . . 248

6.5.1 Development of Techniques . . . . . . . . . . . . . 248

6.5 .2 New Directions . . . . . . . . . . . . . . . . . 249 



\section{List of Tables}

3.1 Example HierSynth execution chains composed of a number of HierSynth trees. Some HierSynth trees are used to generate analysis scenarios and these are discussed in section $3.11(\mathrm{p} 120) . \ldots \ldots$. . . . . . . 118

3.2 HierSynth parallelisation opportunities and challenges within the HierSynth tree execution algorithms, along with current implementation. . . 123

4.1 The effects of particular energy strategies on CommunityB as calculated with Arup's IRM model and a one at a time sensitivity analysis conducted using HierSynth. . . . . . . . . . . . . . . 193 



\section{List of Figures}

2.1 An example visualisation from Arup's 3dUrbanism project [65] integrating design analysis from many disciplines on the scale of a neighbourhood. Buildings are textured with a daylight factor map showing average annual daylight. A Computational Fluid Dynamics (CFD) analysis of the prevailing wind direction is shown by a vector field displayed as arrows. An acoustic analysis of the area is displayed as differently sized spheres. Finally a traffic simulation is shown as an animation representing expected traffic flow. Using the $3 \mathrm{~d}$ environment each discipline can be explored independently avoiding any visual confusion. . . . . . . . .

2.2 The structure of a generic performance tree, showing the use of preference and aggregation functions. Design parameters and analysis results are used as inputs to the leaf nodes of the tree. These are optionally transformed into preference space using preference functions. Working up the tree are KPI's from a single discipline or covering an aspect of design performance are then combined via aggregation functions. Finally at the root of the tree a single score is created through an aggregation function. . . . . . . . . . . . . . . . . 70

3.1 The central challenge that HierSynth aims to address is the lack of synchronisation between the design and analysis cycles in urban masterplanning. As quantified by [35] the analysis cycle can take 5-8 times longer than a design iteration. HierSynth enables exploration of the computational techniques required to resolve these challenges and generate design

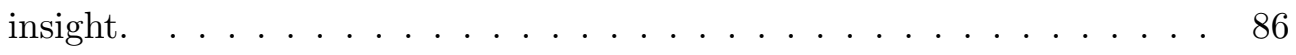

3.2 Overview of HierSynth platform . . . . . . . . . . . . . 87

3.3 A conceptual model of a standard workflow model for optimising a steel structure. The composition of such a model with the design decompositions (see Fig. 3.4 (p90)) often seen in interoperability formats form the basis of the HierSynth data model which is shown in Fig. 3.5 (p91). 
3.4 An example design decomposition of the type often found in interoperability formats within this industry. The composition of such decompositions with workflow data models (see Fig. 3.3 (p89)) form the basis of the HierSynth data model which is shown in Fig. 3.5 (p91) . . . . . . . .

3.5 A conceptual model of a HierSynth tree before (top) and after expansion (bottom). For each initial scenario the tree is executed and the design query is evaluated querying the design for the districts it contains. The sub-tree of each query node is then instanced for each design component (district) returned. In this case an analysis query is then run to compute the carbon emissions from this district. . . . . . . . . . . . . . . . . . 91

3.6 An example variable map displayed as a tree according to the names of its variables. Variable names are of the form "Water_Demand_Commercial_D15_units" or "Water_Demand_Education_D29". This provides a simple ontology and an easy way to navigate thousands of design and analysis assumptions. . . . . . . . . . . . . . . . . . . . 97

3.7 An example HierSynth tree consisting of a number of design queries, several design element templates and an analysis node which also acts as a design element template. . . . . . . . . . . . . . . . . . . . 99

3.8 An overview of the steps for evaluating a HierSynth Tree. From a user composing such a tree, through three steps of the execution algorithm to a final step allowing the user to utilise techniques in the framework for drawing design insight from the analysis results. . . . . . . . . . . 101

3.9 An executed HierSynth tree showing the percapita carbon KPI aggregated at all levels of the design decomposition. This tree is shown after execution, the template for the tree is shown in Fig. 3.7 (p99). . . . . . 103

3.10 A conceptual model of a performance tree for a design component in HierSynth. The tree is based upon analysis results stored in that node (shown in red and as a tree due to the naming convention used in HierSynth as described in Fig. 3.6 (p97)). . . . . . . . . . . . . . . 110

3.11 A Performance tree calculated by HierSynth displaying KPI's from a number of disciplines and aggregating them into composite scores for each area and for the whole building. Colour coding is by performance in relation to peers and enables a quick visual diagnostic of building performance. The perceptive reader will notice lines in the background which are references between multiple performance trees in the design decomposition; this performance tree is just one of around 100 performance trees in a design decomposition breaking a development down into suburbs and districts. Fig. 3.12 (p112) shows this concept. . . . . . 111 
3.12 Conceptual diagram showing the formation of the performance trees for each design component. Each design component's performance tree is formed from KPI's stored on that node (in red) together with the corresponding performance tree nodes in the design component's children. This allows quick drill-down of performance results both in the performance tree, across disciplines and through the design decomposition. . . 112

3.13 This HierSynth tree makes use of cross-links between the SynCity model and the suburb query decomposition. This enables the high-level SynCity analysis to access the energy demand forecasts calculated by the Integrated Resource Management (IRM) model which is run for every building of every type in every suburb. The SynCity model then calculates an optimal resource network and set of power technology to meet these demands. This is shown in Fig. 3.14 (p115) . . . . . . . . . . . . . . 114

3.14 A "worms eye" visualisation of the output of a SynCity Resource Technology optimisation run. Four resource networks are visualised which are providing power to the city to meet the electricity, heating and cooling demands of the city. Interconversion technology (e.g. gas boilers or Combined Heat and Power (CHP) plants) are placed strategically within the city . . . . . . . . . . . . . . . . . . . . . 115

3.15 An example of an executed scenario generation tree. Note that in this case only the leaf nodes will generate scenarios from their variable mappings which will have been patched to include energy scenario data and by their ancestor design node to include design data. . . . . . . . . . . 119

3.16 A simple Execution Chain consisting of a scenario generation tree and an analysis tree. The first generates the composition of designs and energy strategies as 9 new scenarios and the second analyses the design under the scenario with a per-district design decomposition. . . . . . . . . . . 121

3.17 The GUI of HierSynth, showing configuration on the left and the execution of a small HierSynth tree on the right. . . . . . . . . . . . . . . 126

3.18 An example execution chain consisting of two execution trees, one for scenario generation and another for analysis. Into this chain is spliced an experiment generation module for a sensitivity analysis and its corresponding analysis module is used at the end to compute sensitivities. . 130

4.1 Conceptual overview of the application of HierSynth to a live project with Arup North America. Compare with HierSynth overview in Fig. 3.2 (p87). Dashed arrows indicate how HierSynth was used to integrate separate models into a large model adding capacities not available previously. 
4.2 Inputs to Arup's Integrated Resource Management model's percapita carbon calculation. Extracted and classified using the tools developed in Chapter 5 (p209). Note the inputs automated by HierSynth both for analysing different designs and for analysing different energy scenarios. . 147

4.3 This HierSynth tree is used to compose the scenarios under which analyses will be performed. The upper image shows the tree before execution, after execution we see that each query has executed and produced 3 designs and 3 energy strategies. In this case each leaf nodes of the tree becomes a new scenario. Each level of the tree has modified (patched) the scenario with data specific to that design or energy strategy. . . . 150

4.4 Comparison of sources of annual per capita carbon emissions 3 designs under 3 energy scenarios. HierSynth automation enabled this figure to be produced in under ten minutes compared with several hours work previously. . . . . . . . . . . . . . . . . . . 152

4.5 The data table representing the Phasing table which was used to add time as a dimension to the IRM model which previously only analysed at a fixed point in time. Changes in energy efficiency strategies were modelled over construction phases, for example modelling increases in server efficiency over the 10-20 years covered in the construction phases. 154

4.6 Annual operational carbon emissions over 10 construction phases for a high density design under the "Good", "Better" and "Best" energy strategies. Graphing over time was enabled via HierSynth integration, this graph also includes varying assumptions about building codes, emissions factors and energy efficiency over time adding a valuable accuracy and foresight capacity to the model. . . . . . . . . . . . . . . 155

4.7 Annual percapita carbon emissions plotted over time and per district (existing and new). HierSynth enabled modelling of different construction phases and changes in assumptions (e.g. appliance efficiency) over time. This identified trends and coupled with increased detail spotted anomalies such as the one shown here. . . . . . . . . . . . 156

4.8 Conceptual model of data flow between the three Arup models integrated using HierSynth. Integration is discussed in section 4.9 (p157): the IRM model computes Energy Use Intensities (EUIs) for the design without a district energy system, the DEF model then uses these to size several forms of district energy system (e.g. Condenser Water Loop) and the LCCA model estimates the financial cost of the system. From every model HierSynth extracts a set of KPIs as shown by dashed lines. . . . . 157 
4.9 The full data flow diagram of how the integration of the IRM, DEF and LCCA models works. This data flow model is implemented in the HierSynth tree shown in Fig. 4.10 (p161) . . . . . . . . . . . 160

4.10 HierSynth tree template used to implement the integration of models sketched in Fig. 4.8 (p157). This analysis tree was reused to answer many different questions and was used to analyse both the whole development and each district. . . . . . . . . . . . . . . . . . 161

4.11 A per district percapita carbon map for 3 designs under 3 energy scenarios ("Good" is full column, "Better" is middle marker and "Best" is lowest marker). HierSynth automation enabled more detailed insight at the district level to be computed for each design iteration. Without this only the bottom left graph would have been computed which shows results for the whole development. . . . . . . . . . . . . . 163

4.12 DEF energy consumption figures (combined gas and electricity) as calculated from inputs from IRM model under nine design scenarios. Note that the "Good" scenario does not include a district energy system. Scale removed to preserve confidentiality. . . . . . . . . . . . . . . . . . . 164

4.13 LCCA results showing Internal Rate of Return (IRR) for three designs under three energy scenarios. Results are calculated based on inputs from the IRM and DEF analysis models. Note that the "Good" scenario does not include a district energy system. Two implementations of district energy system are used for calculations, 1A is a Condenser Water Loop and 1B adds a Thermal Energy Storage capacity to the system to store excess heat to meet peak demands avoiding investment in expensive additional generation capacity. . . . . . . . . . . . . . 165

4.14 Automated BRE209 [63] daylight analysis of sample geometry for CommunityB project computed using Radiance [103] automated by HierSynth. A false colour scale is used to show illuminance with yellow being full sunlight and dark blue being none. Notice the difference between buildings courtyards as the density of the building changes. . . . . . . 167

4.15 Geometry with BRE209 [63] daylight analysis mapped to grey scale. Block level billboards display information about the sustainability performance of each city block. Interactive exploration of this environment enables identification of problem buildings and performance trends, in this case a red / yellow / green colour scale is used to show water consumption per person per day. . . . . . . . . . . . . . . . . . . . 169 
4.16 A performance tree for one of the blocks in this design. Whilst scoring well for water usage compared to its peers it performs poorly for carbon and electricity, which is not surprising as it consists entirely of old offices which have not been redeveloped in this design. Quickly identifying poorly performing buildings highlights areas for design improvement. . . 170

4.17 The execution chain used for investigating the effects of electric car uptake on the development. This tree produces 99 scenarios (11 uptake levels 3 designs and 3 energy scenarios). . . . . . . . . . . . . . 173

4.18 Effect of Electric cars on operational and transport carbon emissions and on electricity consumption. Figures shown correspond to LU1-Good, LU2-Better and LU3-Best. Note as the design becomes increasingly dense and carbon efficient the need to tackle the transport component of carbon is increasingly clear (see pie charts). . . . . . . . . . . . 174

4.19 Composite energy and carbon costing metric identifies the effect of varying the density of a design under three different energy scenarios. The majority of the benefit of increasing density is achieved by FAR 1.5. . . 177

4.20 Percapita carbon savings with district energy system as the residential proportion of the development is increased. Notice different maxima under different energy scenarios. Expectation was for a strongly " $\cap$ " shaped graph as a balance between building types enables lower peak demand and a more smooth energy demand. Fig 4.21 (p180) identifies part of the reason that this expected trend was not seen. . . . . . . . 179

4.21 Percapita gas consumption with a district energy system as the residential proportion of the development is increased. Notice how the capacity of the system negates the need for gas consumption in the development up to the point where additional technologies are required. . . . . . . 180

4.22 Percapita electricity saving with a district energy system as the residential proportion of the development is increased. Note that the intersection points with the zero line, match the points of inflection shown in Figs. $4.20(\mathrm{p} 179)$ and $4.21(\mathrm{p} 180)$. . . . . . . . . . . . . 181

4.23 Transportation modal splits as residential as percentage of development that is residential increases. Note " $\cup$ " shape in the most heavily polluting

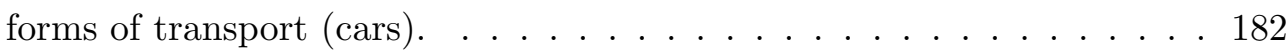

4.24 Percapita transport carbon varying with the residential fraction of the development. Unexpectedly we see an " " shape rather than the ex-

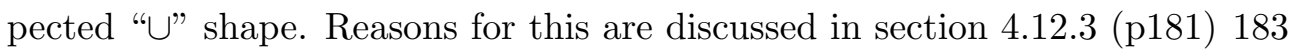


4.25 These graphs explore the trends identified in Fig.4.24 of the impact of residential / commercial mix on transportation carbon emissions. Note that scales have been removed to preserve commercial confidentiality. Graphs c \& d share the same scale. Graphs e \& f also share a scale. . . 184

4.26 Effect of adding residential GFA into the development upon operational carbon per annum. Note that most of the gain from adding residents is acheived by adding 35\% extra residential GFA corresponding to the $30 / 70 \%$ residential/commercial sweet point identified in section 4.12 .3

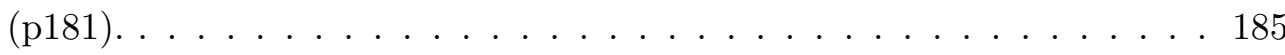

4.27 Effect of adding residential GFA into the development upon carbon savings achieved by adding a district energy system. Trends should be compared to the first half of Fig. 4.20 (p179). . . . . . . . . . . 186

4.28 Sensitivity analysis results for the operational carbon percapita KPI. We normalise results to the most impactful variable. Over 1,200 variables were analysed as part of this analysis. . . . . . . . . . . . 188

4.29 Sensitivity analysis results for the waste water per annum KPI. We normalise results to the most impactful variable. It is also possible to consider side effects upon other KPIs; over 200 of which were considered. . 189

4.30 The execution chain used to investigate the impact of 60 energy strategies across three designs and three energy scenarios. The first tree composes these 9 scenarios (see Section 4.6 (p148)), next scenarios are created which turn each energy strategy on one at a time, finally the integrated analysis tree developed in section 4.9 (p157) is used to analyse these 549 scenarios. The left of this figure shows the user interface for creating and configuring execution chains. . . . . . . . . . . . . . 192

4.31 The most effective energy strategies in the IRM model for effecting the "Total Cash Operating, Thermal \& Electricity Costs" of a proposed district energy system for the CommunityB development. This KPI was chosen from 1,315 computed across the integrated model ensemble developed in section 4.9 (p157); sensitivities were computed under all nine scenarios generated in section $4.6(\mathrm{p} 148) \ldots \ldots \ldots$. . . . . . . 195

5.1 Conceptual model of an Integrated Resource Management (IRM) Model [12]. Sustainability models from many disciplines are integrated to form a coherent model for assessing urban masterplans [75] . . . . . . . . . . 210

5.2 Dataflow through Arup's IRM model [75]. The complexity of multidisciplinary engineering models and difficulty of comprehension makes project adaptation and optimisation tasks challenging. . . . . . . . . . 212 
5.3 Arup's IRM model [75], is implemented as a Microsoft Excel spreadsheet. Each discipline has an input and an output model in its own worksheet. A single project metrics dashboard is provided. The IRM model contains over 20 submodels and some versions of the model contain over 50,000 calculation cells. . . . . . . . . . . . . . . . . . . . . 213

5.4 Visualisation demonstrating the complexity of the calculation graph of a small subset of Arup's IRM model. Nodes are calculations and edges show dataflow. . . . . . . . . . . . . . . . . . . . .

5.5 Graph showing a model slice of the calculation of the $\mathrm{CO}_{2} \mathrm{e}$ emissions percapita per annum for external transport. Layout highlights the calculation for ten modes of transportation. . . . . . . . . . . . . . . . 218

5.6 Per discipline metrics calculated from a calculation graph extracted from a model slice for annual percapita carbon emissions. Each discipline has an input and an output model, the \% input column shows that over time some inputs have been entered in output models. Average valency is the average number of cells each cell references and is a measure of calculation complexity. . . . . . . . . . . . . . . . . . . . 220

5.7 Discipline coupling matrix shows discipline communication in the IRM model. Matrix should be read " $x$ values in row model are used by column model". For example 53 values in the Land use model are used in the Energy Demand output model (SS_ED). Circular markers show indirect couplings (references made to a discipline which references another discipline). . . . . . . . . . . . . . . . .

5.8 Software engineering metrics normally applied to large software projects [67] are applied to multidisciplinary models to gain insight into model maintainability and stability to change. . . . . . . . . . . . . . . 223

5.9 Frequency plot of the length of 50,000 formulas encountered in the IRM 2011 model. Note the large number of formulas over 100 characters which proved very difficult to maintain. . . . . . . . . . . . . . 225

5.10 We applied the EAM [61] toolkit to three different IRM models ranging from a concept model to a fully developed model to a globally used geographically localised tool. The increase in complexity is down to increased use of data tables localising the model to a region. . . . . . . . 227

5.11 A sensitivity analysis identifies the variable with most scope to impact a KPI. We normalise to the most impactful variable. We show impact upon total percapita carbon and side effects on some constituent parts of the total percapita carbon figure. We analysed over 900 input variables.230 
5.12 Per discipline metrics calculated from a calculation graph extracted from a model slice for annual percapita carbon emissions from the earlier IRM concept model. Compare with Fig 5.6 (p220) to see model development. 233

5.13 Discipline coupling matrix shows discipline communication in the IRM Concept model. Matrix should be read " $x$ values in row model are used by column model". For example 24 values in the Land use model are used in the Energy Demand Supply output model (SSEDES). Circular markers show indirect couplings (references made to a discipline which references another discipline. Fig 5.7 (p221) shows how discipline couplings changed as the IRM model was evolved. . . . . . . . . . . . . . 234 



\section{Introduction}

Urban masterplanning is the process of designing an urban area, from redeveloping a business park to creating a new city suburb. The range of disciplines and skills involved in creating successful developments for all stakeholders makes urban masterplan design and analysis a challenging field. It is a high-level activity setting the overall shape of the development and defining development targets, standards and maximum envelopes for each building plot rather than designing each building in detail at the outset. Quantitative analysis is undertaken by various engineering disciplines to benchmark designs on metrics such as carbon emissions, energy demand and consumption, transport performance, daylight, windflow and so forth; giving practical insight into the design. It is also the design stage with perhaps the most scope for improving a development's sustainability and performance.

Within the urban masterplanning process there is currently a substantial gap between the design and analysis cycles. An analysis cycle takes many times longer than a typical one week design iteration [35]. Thus masterplanning projects will have only one or two quantitative analysis cycles despite many times this number of design iterations occurring without analysis insight. Hence analysis cycles are often used to ensure compliance with regulations rather than as a key design improvement tool. Additionally in design competitions, due to their short time scale, it is not uncommon for analysis to be omitted altogether. This can lead to commitment to designs which are inherently poorly performing.

Underlying this are a number of organisational and computational challenges. Most notably there is little automation within the Architecture Engineering and Construction (AEC) industry. This leads to limited model integration and much manual reworking of data. Limited computational skills within the industry also mean that scaling analysis to cover every building in a development is a substantial challenge. So too is managing the data generated by analyses. Together these challenges mean that very limited quantitative design space exploration is undertaken, precluding design improvement.

Within the AEC industry, research has aimed to address these challenges either by incorporating the design cycle into the analysis cycle as part of an optimisation loop (see section 2.5 (p56)) or by using simplified analyses directly within the design cycle (see section 2.6 (p62)). However neither approach has considered the underlying computational frameworks required for unifying the two sides of urban masterplanning. 
Computational Design Optimisation (CDO) research such as [17] [89] [102] aims to automate the two cycles. However, whilst producing improved design and insight they are limited in breadth of application and remove the best sources of insight from the loop, i.e. the practitioners. Tightly coupled design and analysis environments such as [5] [10] [49] allow designers rapid feedback upon their designs, however they frequently lack the flexibility to support the breadth of design disciplines and the capacities to support design space exploration. Finally, approaches such as Building Information Modelling (BIM) [31] and numerous industry interoperability standards such as [55] [37] [22] have tried to ease these computational challenges. However these are each too narrow to cover the broad urban masterplanning discipline and do not consider how to resolve the underlying mismatch between the design and analysis cycle.

This thesis aims to address this challenge from a computational perspective. This is done by creating a unifying computational platform "HierSynth" which brings together the design and analysis cycles though a compositional data model. This creates design decompositions using design queries against design geometry and land-use schedules. These are then annotated with analyses to be automatically run using industry standard tools such as Radiance [103] for lighting analysis and Arup's Integrated Resource Management Model [12] for sustainability assessment. HierSynth enables previously infeasible, practitioner driven, design space exploration through scenario composition and sensitivity analysis. HierSynth acts as a test-bed for exploring the efficacy of computational techniques in an urban masterplanning context. These techniques are drawn in part from the success of computational product design frameworks within the aerospace and automotive industries [80] [27] [29]. These techniques range from automation, model integration, data management, workflow management, scenario generation and techniques for design space exploration.

The key contribution of this thesis is the reconceptualization of the workflow graph by composing it with tree based design decompositions more commonly found in industry interoperability file formats. This is achieved through a hierarchy of design queries, templates and analysis which; when combined through a novel dataflow algorithm form, when executed, a design hierarchy annotated with analyses to be performed. This enables simple multi-scale analysis at all levels of detail, a key feature for use in the realm of urban masterplanning. This data structure is then reconceptualised to provide scenario generation capabilities and used sequentially allow the specification of design space exploration experiments to answer the "what if" questions frequently asked by engineering teams. This framework combined with techniques including sensitivity analysis, performance trees at every level and integrated $3 \mathrm{~d}$ visualisation, are applied to the discipline of urban masterplanning to bridge the growing computational gulf between architectural design and analysis teams.

The HierSynth platform is evaluated by applying it to a live urban masterplanning 
project with Arup North America. This case study generated much design insight for the practitioners. Firstly by automating the running of complex analysis models. This reduced the analysis cycle to less than a day enabling some 23 analysis iterations - an order of magnitude more than literature would suggest is normal. Secondly HierSynth was used to augment existing models by modelling changes in analysis assumptions over time to give more accuracy. Similarly by replacing manual model integration with an automated model ensemble HierSynth was able to produce more accurate results with greater analysis capabilities. Finally, these capabilities were used to study design space exploration questions which would previously have been computationally infeasible to answer. These include exploring the optimal mix of residential and commercial development for a district energy system. Many of the capabilities are novel within this field.

The insight gained during this case study helped draw conclusions on the most effective computational techniques and highlight further requirements for computational support within urban masterplanning. Most notable among these was the need for tools to comprehend, audit and debug complex engineering models implemented as spreadsheets. This need is explored within this thesis by creating a methodology and toolkit for statically analysing and auditing complex engineering spreadsheets. These identified several bugs and implementation issues in an engineering model as well as giving insight in model construction and evolution. The methodology was used successfully to give insight to practitioners on model capabilities and support them in reducing predicted carbon emissions calculated by the case study model.

In summary this thesis considers the field of urban masterplanning as ripe for the application of revolutionary computational unification. This thesis serves as an introduction to and evidence of the challenges and solutions involved in computationally unifying urban masterplanning. From this research an underlying thesis has emerged, namely that:

This thesis contends that the key to generating quantitative insight for practitioners within urban masterplanning is the unification of computational techniques such as analysis automation, model integration, scenario composition and sensitivity analysis in a single framework to provide multi-scale insight by through design space exploration and detailed design performance metrics. 


\subsection{Contributions}

The contributions of this thesis are set out as follows:

1. We present a reconceptualization of a workflow graph by composing it with design decompositions more commonly found within architectural design formats. This is achieved through a tree based specification of design queries, templates and analysis nodes combined through a novel dataflow algorithm. This enables detailed insight and multi-scale analysis at every level. Such trees are then reconceptualised to generate scenarios for design space exploration. These features combine to address many of the computational needs of the urban masterplanning community and are implemented in the HierSynth framework.

2. We present a novel computational framework (HierSynth) that supports the composition of design queries, analysis models and so-called 'performance trees' together into a hierarchical model that supports design decomposition, scenario generation and composition, workflow automation, model integration, sensitivity analysis and user-driven design space exploration. The framework provides a foundation for exploring the efficacy of computational techniques in unifying urban masterplanning and producing design insight.

3. We present a detailed evaluation of the HierSynth framework and its data model on a major commercial urban masterplanning project, in conjunction with Arup North America. This enabled exploration of the benefits of computationally unifying urban masterplanning and evaluation of the efficacy of the computational technique employed. This proved the framework can enable an order of magnitude more analysis cycles than normally feasible. The framework enabled investigations such as identifying the optimal mix of residential and commercial development for district energy system performance and reduced transportation carbon emissions. The case study revealed requirements for adopting version control and provenance tracking for design files and analysis models and, in particular, aids for investigating and debugging complex spreadsheets which form the basis of many analysis models.

4. We present a general tool suite that supports the systematic, automatic analysis of large spreadsheet-based models, and a methodology for using such tools to enhance the model's quality and value. Large spreadsheets are used in many disciplines, but are particularly important here as they are often the tool of choice in the analysis of urban masterplanning projects. The tool suite is evaluated by exploring three generations of Arup's Integrated Resource Management (IRM) Models at different stages of development, but the potential applications of the tools and associated methodology are much more general. 


\subsection{Structure of Thesis}

This thesis explores the following research questions:

1. What computational challenges face the urban masterplanning design and analysis cycles and particularly their intersection?

2. Which computational frameworks and techniques are necessary and effective in creating design insight in the context of urban masterplan design and analysis?

To investigate these a program of research is reported in this thesis as follows:

- In Chapter 2 (p37) discusses the computational challenges facing the urban masterplanning community and current approaches to addressing these challenges and the techniques employed to generate insight.

- In Chapter 3 (p85) a computational framework "HierSynth" is developed to investigate a novel data model and a number of techniques which may help address the challenges facing urban masterplanning.

- In Chapter 4 (p139) a collaborative application of the HierSynth framework to an urban masterplanning project is undertaken with Arup North America. This enables investigation of the efficacy of the data model and techniques within HierSynth. A number of unexpected challenges are also identified during this collaboration.

- In Chapter 5 (p209) one of the most pressing of these challenges is explored via the development and application of a methodology and toolkit to help with the comprehension and debugging of complex engineering assessment models implemented as spreadsheets.

Finally in Chapter 6 (p237) conclusions are drawn as to how well this thesis can be supported, which techniques and data models are most effective at generating insight to draw together the contributions of this thesis and discuss further investigations.

\subsection{Publications}

The research described in this thesis has been published as follows.

\subsubsection{HierSynth}

The story of computationally unifying urban masterplanning is told within the following publications. 
- "(Computationally) Unifying Urban Masterplanning Design, Analysis \& Evaluation" David Birch, Paul Kelly, Tony Field, Alvise Simondetti - Poster.

- Presented at "Google PhD Fiesta" 20th March 2012 Dept Computing Imperial College. Awarded "Best in Year" (third year).

- Presented at "Graduate School Summer Research Symposium" July 2012 Imperial College London.

- This poster is included as the graphical abstract of at the start of this thesis.

- "HierSynth - Automating City-Wide Architectural Analysis for Design Insight" David Birch - Paper \& Presentation - Arup Doctoral College Conference 2011 [14].

- Introduces the HierSynth platform and the challenges it seeks to resolve. It then describes the first third of the case study in Chapter 4 (p139) namely automating current practice to unify urban masterplanning analysis and design.

- "Computational Investigations in Masterplan Design" David Birch - Paper \& Presentation - Arup Doctoral College Conference 2012 [15].

- Describes computational design space explorations undertaken in Chapter 4 (p139) and the design insight they generated - most notably exploring the optimal mix of commercial and residential development for optimal district energy systems performance and reduced transportation carbon emissions described in Section 4.12 .3 (p177).

- "Computationally Unifying Urban Masterplanning" David Birch, Paul H. J. Kelly, Tony Field, Alvise Simondetti - ACM Computing Frontiers May 14-16 Ischia Italy 2013 [16].

- Describes the computational challenges faced by the urban masterplanning community and the approach taken by the HierSynth platform to address them together with the evidence and lessons learned during the case study of Chapter 4 (p139).

In this thesis this work is presented as follows: The difficulties faced by urban masterplanning and current approaches are described in Chapter 2 (p37). The HierSynth framework and approach to resolving these challenges is described in Chapter 3 (p85). The case study and practical evidence of the efficacy of techniques and approach is described in Chapter 4 (p139). Conclusions drawn from these investigations can be found in Section 4.15 (p199) and Chapter 6 (p237). 


\subsubsection{Spreadsheet Analysis}

The methodology and tools created to explore complex engineering models implemented as spreadsheets is presented in the following publications. In this thesis this work is described in in Chapter 5 (p209). This work was the result of a collaboration with Helen Liang of Bath University.

- "Extraction and analysis methodology for supporting complex sustainable design" H. Liang and D. Birch - Paper \& Presentation - 18th International Conference on Engineering Design (ICED11) Copenhagen, 2011 [61].

- "Supporting Complex and Sustainable Ecocity Design Using Extraction and Analysis Methodology (EAM)" H. Liang \& D. Birch - Paper \& Presentation - Ecocity World Summit, Montreal 2011 [62]

- "Extraction and Analysis Methodology for Supporting Complex Sustainable Design" David Birch, Helen Liang, Joan Ko, Alvise Simondetti, Paul Kelly, Tony Field, Glen Mullineux, Geoff Hammond - Poster

- Presented at "Google PhD Fiesta" Dept Computing Imperial College March 2011. Awarded "Best in Year" (second year).

- Presented at "Graduate School Summer Research Symposium" Imperial College London 2011.

- Presented at "Energy Efficiency in the Built Environment: A Business Opportunity" London Technology Network November 2011.

- "Examining Excel Evolution" David Birch \& Helen Liang - Poster - Arup Doctoral College Conference 2012

- "Multidisciplinary Engineering Models: Methodology and Case Study in Spreadsheet Analytics" David Birch, Helen Liang, Paul H J Kelly, Glen Mullineux, Tony Field, Joan Ko, Alvise Simondetti - Conference Paper \& Presentation - European Spreadsheet Risks Interest Group (EuSpRIG) 2013 Greenwich London .

\subsubsection{Industry Vision}

A series of three vision papers [93] [94] [95] on the future of Built Environment Modelling have been produced by Alvise Simondetti of Arup Foresight and Innovation. These are available online at http://www. driversofchange.com/make/tools / future-tools/ and have been published as follows:

- "BEM for Collaborative Design Inception: Harnessing the Power of Clients' Design Intuition" Alvise Simondetti, Simon Roberts, David Birch - proceedings 
of The 2012 International Conference on Modeling, Simulation \& Visualization Methods [91].

- "Digital Environments for Experiential Design: Enhancing Designers' perception" Online vision paper [95]

- HierSynth and the case study visualisations discussed in Chapter 4 (p139) are featured as a case study with particular emphasis on the visualisations carried out in Section 4.11 (p166).

- "A Practical Perspective on Computer Tools for Sustainable Building Design" A. Simondetti, S. Roberts \& D. Birch Proceedings of the 2012 International EG-ICE Workshop on Intelligent Computing, Herrsching, Germany. Key Note Talk \& Extended Abstract [92]. 


\section{Techniques for Computationally Unifying Urban Masterplanning}

This chapter explores the challenges in urban masterplanning design and analysis and the techniques and methods currently used to address them.

The field of architecture and architectural analysis is substantively different from other engineering and design disciplines. As such it faces a unique set of challenges to improving the performance, efficiency and capacity of the design process. In this chapter we will explore how many of these challenges have computational origins and/or solutions. We will first explore these challenges and their reasons before exploring current techniques and research for resolving these challenges.

\subsection{Research Fronts}

Research into the application of computation to the Architectural Engineering and Construction (AEC) industries is varied and covers several aspects. First there is discipline-specific research in terms of better digital design tools for architects with developments including digital drafting and design and more recently parametric design tools such as Rhinoceros [84] and Grasshopper [41] which aim at increasing the expressibility and flexibility of architectural design. Similarly each engineering discipline continues to create more accurate, more flexible analysis models with which to analyse the increasingly complex architecture being produced. This has created a computational "arms race" between architects and engineers for modelling flexibility and accuracy to cope with ever more unique and complex geometry at ever higher levels of performance across a wide range of metrics (e.g. cost, lighting, structural stability, construction speed...). This has created a growing gulf between each of the design and analysis disciplines consisting of computational interoperability challenges, capabilities and differing lengths of analysis cycle.

These challenges include a lack of automation caused by limited APIs and file format interoperability problems. These are compounded by a lack of scripting skills which results in a lack of analysis scale (e.g. analysing 200 buildings is hard). Similarly there are limited resources to handle large repositories of analysis results and designs and particularly the tools to produce insight from large quantities of analysis results. These challenges and their causes will be discussed in section 2.2 (p39) with a particular 
focus on computational challenges in section 2.3 (p47). Such challenges have made rapid quantitatively analysed design cycles difficult to achieve with an average project containing only two or three quantitative iterations [35]. This lack of iteration prevents design improvements which could be obtained through multiple quantitatively-analysed design iterations.

Within the aerospace and automotive industries a range of computational support over the last few decades has generated an range of design-analysis platforms for use in product development [29] [27] [80] which address many similar challenges within these industries. Whilst a few attempts (e.g. [36]) have been made to apply these systems to the architectural context many additional challenges are faced. For example allowing design decomposition for per component analysis, or supporting collaborative working by many practitioners from many disciplines each with their own models. Section 2.4 (p51) considers the techniques these systems employ and their adaptability to the AEC industry.

Taking these systems as inspiration a wide research front has grown looking into Computational Design Optimisation (CDO) aiming to automate the process of finding the optimal design and engineering to meet specific cost, structural [102] and more recently qualitative considerations [17]. These systems combine an optimisation algorithm (normally a genetic one) with parametric or procedural design tools to generate design alternatives. A number of analyses are then used to benchmark the design to provide a fitness function to the genetic algorithm. A number of these approaches will be discussed in section 2.5 (p56).

Finally there have been several areas of research tackling particular challenges faced by the industry. Most notably that of interoperability where two approaches have grown. The first approach uses interchange file formats such as the Collada format [55] for digital files, Industry Foundation Classes (IFCs) [22] for building models among others [37] [42]. The second approach uses a more active server based collaborative repository approach as shown in the rise of Geographic Information Systems (GIS) and Building Information Modelling (BIM) databases and collaboration systems, the second of which has generated a wide literature with many outstanding challenges [32]. Section 2.8 (p71) discusses these approaches and draws conclusions for successfully mitigating the challenges.

Despite this breadth of techniques and approaches a research question still remains open, namely how to bridge the gap between the design cycle and the analysis cycle within the urban masterplanning community and which techniques are most effective at generating design insight in such a system. The majority of the techniques discussed in this chapter are not targeted specifically at urban masterplanning design and analysis. This thesis aims to explore which computational techniques and models are required and effective at generating design insight and resolving existing challenges in urban 
masterplanning. Several recent successful projects employing CDO techniques [89] have demonstrated the benefits of resolving these computational challenges. However this thesis demonstrates that more valuable than design optimisation techniques are approaches to provide design and design space insight to the design and analysis teams of which CDO is just one.

As examples to the reader, design insight in this field is varied and ranges from identifying problems such as:

- Quantifying the performance of the design.

- Identification of the worst-performing districts in a masterplan.

- Comparison of different design alternatives for example using different densities.

- Exploration of the effects of design strategies such as district energy systems.

- Finding the optimal mix of residential and commercial development.

- Ensuring the design meets certain minimum performance criteria.

In this chapter we will explore the computational challenges faced by the AEC industry with a focus upon urban masterplanning in section 2.2 (p39); drawing a list of computational challenges in section 2.3 (p47). We will then consider in section 2.4 (p51) a number of engineering design optimisation systems used in the aerospace and automotive industries with considerable techniques and identify a number of common techniques used to address these challenges. We will then review the techniques used in the more recent architectural CDO systems for generating design insight in section 2.5 (p56). We will then consider in section 2.8 (p71) a number of techniques addressing particular challenges identified in the AEC industry such as interoperability together with examples of techniques likely to be useful within the AEC industry such as visualisation in section 2.7 (p66). Finally this chapter will draw together in section 2.10 (p80) a set of hypotheses for effective computational techniques for use resolving the challenges faced by the industry.

\subsection{Architectural Industry Challenges}

In order to give the reader a background in this industry we explore the unique challenges faced by the AEC industry. We then seek to extract the implications and opportunities for computation to help resolve these challenges. In the following section we discuss these computational challenges in more depth and focus upon the challenges which will be address through this thesis. Such challenges are often the reasons why this industry has not adapted the computational toolkits commonly employed in other more computationally advanced product design automotive and industries (e.g. aerospace) 
which are used to carry out automated quantitative design assessment rapidly enough to provide feedback during the design cycle resulting in higher performing designs.

- Fragmentation of the industry - Each stage of the commissioning, design, analysis, construction and operation lifecycle will frequently be undertaken by different companies and will involve many different disciplines. In addition it is very common for different design disciplines to be subcontracted with civil engineering, infrastructure, landscaping and transportation planning amongst others not only being different disciplines but being undertaken by different firms. Finally even within a single design stage it is likely that analysis will be undertaken by many firms with transportation, fluid dynamics and other disciplines being outsourced away from architectural design firms. This level of fragmentation differs from aerospace where one company will control many of these stages without barriers of communication, contracting and confidentiality. These barriers will reduce communication, design and analysis iteration and speed of modelling. Within this fragmentation we see specifically

- Design / Analysis - Since each analysis cycle will be a new contract item which must be paid for there is limited financial incentive for many cycles of quantitative design improvement beyond satisfying the client with the design, its performance and ensuring it meets regulatory and planning permission constraints.

- Analysis / Subcontractors - Frequently different analysis disciplines such as carbon assessment, energy demand and traffic modelling will be contracted to different companies which makes cooperation in assessment difficult. This is a key challenge to the cooperation which is key to accurate and rapid analysis given the highly interrelated nature of design assessment and particularly carbon assessment.

- Design / Build - The architects and analysis engineers will frequently not be in charge of the construction process beyond producing design documentation and an oversight role. This reduces feedback on construction problems and incentives to improve the design performance in light of feedback gained in the construction process.

- Build / Operate - A widely acknowledged problem within the industry [64] is the lack of post occupancy performance data from constructed designs. Even where performance data, for example in energy consumption, is present, there is frequently an order of magnitude error between collected data and the original analysis results.

- Lack of design feedback - Stemming from this fragmentation an architect will 
normally receive limited feedback on their design particularly once it is built and in regular use.

\section{Computational Requirements:}

- Collaborative working environment enabling feedback on designs from multiple companies and stakeholders in a timely and insightful way.

- Security of confidential data, particularly when multiple firms are involved within a project.

- Tracking of work done across and within firms for accurate billing and completion of contracts.

- Data sharing framework with requests for data and provenance tracking. This would support collaboration by ensuring the data required for analysis is shared correctly.

- Integration of operational sensor data into analysis models. This would help close the gulf between architectural design and engineering and the building managers who operate the buildings.

- Bespoke design - Almost every architectural design will be unique. This is not only due to the artistic nature of architecture but primarily due to the unique opportunities and constraints of each design which will vary with location, client and planning regulations. This is a substantial difference from the automotive industry where each model of car will be an improvement on its predecessor aiming to meet the same challenges to a better degree. This also limits the transferability of any feedback from the design and construction process. This is a particularly acute problem in urban masterplanning due to the limited number of such projects and their uniqueness. In addition the timescale of an average masterplanning project can be several decades from initial design feasibility studies and design competitions to the completion of construction and many projects are never fully completed. This means that feedback cycles are very slow and may occur only a few times within a career. 


\section{Computational Requirements:}

- Robust and adaptable computational analysis under multiple scenarios to cope with bespoke designs and unusual engineering.

- Manage design and analysis results and databases within and between projects to aid transferability of insight by trying to extract common lessons from hundreds of bespoke designs.

- Analysis which is simple to employ and provides valuable design insight.

- Design competitions - Frequently large architectural projects and particularly urban masterplanning projects will be put to a design competition where architects will submit concept designs for the development which will then be used to choose the preferred urban masterplanner. These designs are normally only completed at a high level, without quantitative design assessment and often without involvement of technical disciplines. Hence these concept designs are likely to perform poorly in terms of sustainability, cost of construction, energy consumption and so forth. The challenge this presents is that the design competition submission will then shape the broad shape of the subsequent design and limit opportunities for improving design performance since many design decisions are already fixed by the concept design.

\section{Computational Requirements:}

- Analyses which support concept level design with quantitative analysis results, particularly support analysis based on the limited information available during concept design.

- Analysis cycles which are quick enough to respond to the short time scales and often daily evolving designs during a design competition.

- Tools to support increasing design detail as the design progresses; particularly avoiding needing to redraw of change analysis program.

- Limited participatory design - The design process often doesn't involve the client directly but rather consists of iteratively understanding requirements and presenting a solution. More potential value is to be gained for actively exploring the design space with the client [93] and understanding the costs and benefits from each direction the design could move in. 


\section{Computational Requirements:}

- Tools for effectively communicating design performance to the client and other stake holders.

- Tools presenting quantitatively-analysed design options to the client in an intuitive manner allowing design space exploration in client-designer meetings.

- Rapid enough design / analysis cycle for use between or even within client meetings [95] [76].

- Number of disciplines - A typical masterplan assessment may include feedback from transport planners, carbon assessors, Heating Ventilation and Air Conditioning (HVAC) system designs, lighting design, wind modelling, energy demand modelling, energy supply modelling, PhotoVoltaic (PV) specialists, water planning, electrical planning, gas planning and many more. This range of experts is not normally required in product design nor within the normal optimisation studies frequently undertaken in more computationally advanced product design industries such as the defence, automotive and aerospace industries.

\section{Computational Requirements:}

- Ability to support many different disciplines analysis engines.

- Improved interoperability with many different file formats used by a variety of disciplines.

- Enable integration of different analysis models to support multidisciplinary analysis workflows and investigations.

- Regulatory Approval - while car and plane design will need to meet certain safety, emissions and road tests they do not face regulatory oversight of their design in the same way that an architect must obtain approval of their planning authorities covering aspects including aesthetics. Until recently there were limited regulatory requirements for design performance in terms of energy consumption and carbon emissions which focused the design process on other aspects of the design which didn't require quantitative assessment. 


\section{Computational Requirements:}

- Ability to rapidly and automatically check if a design meets regulatory standards and performance targets.

- Ability to communicate design performance to general public (particularly in comparison to worse performing but more traditional designs).

- Ad-hoc custom models - Many analysis disciplines use ad-hoc analysis models created by practitioners to assess designs. These models are frequently created as spreadsheets or custom scripts to interact with more well-known analysis software.

\section{Computational Requirements:}

- Flexibility to integrate and automate ad-hoc models and scripts.

- Fragmentation of Software - With a few exceptions most design and analysis software systems are separate entities and limited focus is put on interoperability requiring frequent re-construction of the same design in different software (e.g. to provide airtight geometry needed for an airflow analysis). Similarly different analysis software doesn't interoperate well. Whilst there are standardised formats for some disciplines within the industry these are often narrowly focused. Differing focus and data layout compounded by poor tool support make moving between formats challenging.

\section{Computational Requirements:}

- Ability to support many interoperability formats.

- Ability to handle conversion between file formats.

- Ability to transform geometry into the required formats for different analyses and disciplines.

- Extensibility to support many design and analysis programs.

- Urban Masterplanning Analysis Scale - Comparing urban masterplanning with the more typical building design we see a large increase in scale, for example a masterplan may contain dozens of city blocks each containing many buildings. Current most architectural analysis models do not offer the capability to support analysis on multiple scales. Instead one model is used for the whole development while another is used on a per building scale. However other than custom 
scripting there are no tools supporting the analysis of hundreds of buildings individually. Additionally the breadth of scales is also a challenge; particularly when considering implications across scale levels. For example an architect may be interested in the maximum height of a particular building to ensure right to light for its neighbours, but at the same time be concerned with the overall city scale potential for employing solar panels across the development and so will wish to avoid overshadowing.

\section{Computational Requirements:}

- Ability to scale analyses to larger designs. For example automatically analysing hundreds of buildings in a design rather than a limited number manually.

- Ability to exploit levels of detail as the design develops for analysis and visualisation. Enabling the same analyses to be used for both the concept, masterplanning and detailed design stages of design.

- Ability to manage analysing hundreds of separate design elements, carefully collating analysis results for subsequent analysis.

- Ability to manage multiple analysis scales and enable integration between different analysis scales.

- Speed of Analysis Cycle - As discussed in [35] and confirmed in discussion with Arup practitioners the analysis cycle for a design is frequently substantially longer than the design cycle. This has a number of effects including out-of-date analysis results being used to inform decisions and design iterations occurring without quantitative assessment. This frequently results in analysis being used only to show regulatory compliance of the final design rather than as a tool for design improvement. Partly this is due to the challenges identified previously, particularly interoperability problems and the fragmentation of disciplines and software between companies. One important thing to understand here is that the analysis "results" returned to the client may not simply be the output of the software but a large report discussing the analysis results and the analysts investigations into the design, its performance and options for improving this. Thus there are two challenges and potential benefits here. Firstly the chance of improving the speed at which analysts can return simple analysis results in a format comprehensible by the client. Secondly a requirement for improving the capacity and crucially the speed at which the analyst can undertake their investigations into design performance and potential improvements. We note 
from discussions with engineers a positive connection between the two, in that improving simple analysis efficiency will allow more time for the engineer to focus on their investigations which is the part which they can provide most value to their client.

\section{Computational Requirements:}

- Ability to rapidly analyse a design to provide more immediate insight to improve the next design cycle.

- Ability to support computational investigations into design performance under many scenarios.

- Ability to automatically produce deliverables of direct use to clients and to engineers (e.g. graphs and visualisations).

- Challenges of Retrofit - 80\% of the buildings that will stand in 2050 in the UK are already built [58]. Many architectural design challenges and particularly urban masterplanning projects will contain a substantial retrofitting challenge - whether to rebuild or retrofit, which strategies and technologies to employ to what degree. Gaining data on existing buildings and their performance can be challenging as can analysing the potential impacts of interventions upon these buildings.

\section{Computational Requirements:}

- Ability to seamlessly handle many sources of design data (e.g. existing conditions and retrofit design).

- Ability to combine analyses to identify effects of retrofit on existing buildings.

- Ability to incorporate sensor and social data into analyses to support assessment of the impact of retrofit strategies.

- Computational Skills - Within the AEC industry there is limited formal training in computational skills. Whilst many users interact daily with complex design and analysis tools few users have formal or informal training in scripting or data handling. Those who do are often younger in the industry and lack the experience and intuition of discipline experts [66]. This mismatch can result in poor design. Enabling wider use of scripting and automation could give many benefits to this industry should this skills gap be bridged. 


\section{Computational Requirements:}

- Simple user interfaces, with shallow learning curve that still enables users flexibility.

- Leverage existing models and skills; for example exploiting the widespread use of Microsoft Excel and its formula language.

These challenges show the unique challenges faced by the architectural design and analysis industry. This thesis explores how many of these challenges can be at least partially addressed by better computational support. However given the limited adoption of computational support it is likely that the best computational techniques have not been formulated or applied to the area. We will now consider the computational requirements for unifying urban masterplanning design, analysis and evaluation before considering the computational techniques currently applied to address similar challenges in this industry and in the more computationally mature aerospace and automotive industries.

\subsection{Computational Research Challenges}

Having discussed the challenges the AEC industry faces we now explore these challenges both in terms of computational engineering needs and research challenges. Considering that each of these challenges contains both an engineering and a research component. We then conclude by focusing this thesis upon a selected subset of the research challenges identified.

- Improve Analysis Speed - for quicker feedback enabling more analysis iterations and quantitative design improvement. Substantial benefit could be gained from improving analysis speed such that each design iteration is analysed. Similarly a fast enough analysis cycle to respond within the window of a design decision would give substantial scope for improving design performance at a critical stage. Finally should analyses be fast enough for use within client meetings as new options are discussed and a coarse analysis performed for real time feedback the design process could provide many benefits [93] [76].

- Engineering Issue - Use software performance optimisation techniques to improve the speed of analyses (such as parallelism and caching).

- Research Question - How can workflow automation platforms be adapted or created to target specifically the urban masterplanning community to improve integration between the design and analysis cycles with a faster analysis cycle. 
- Support Collaboration - allowing simpler multi-disciplinary work, ameliorating concerns with sharing data such as confidentiality and tracking work done to enable accurate billing. Providing simple communication between disciplines, designers and clients.

- Engineering Issue - Create web based platforms which enable collaboration, data sharing and communication surrounding design and analysis files.

- Research Question - Identify how to track provenance of data, model and analysis updates and contractual obligations within this industry.

- Support Analysis at Scale and in Detail - Enable practitioners to produce better performing designs involving hundreds of buildings; each analysed using detailed models normally only used on a single building. Manage this data and identify means of highlighting useful design insight. Support different scales of analysis and analysing all design components with analysis at their scale and level of detail. For example running an energy demand model for each building followed by a development wide district energy system optimiser [53].

- Engineering Issue - Provide computational frameworks to setup, run and manage analyses.

- Engineering Issue - Provide simple management of design and analysis data across many dimensions of the design space and design decompositions.

- Engineering Issue - Utilise level of detail support within CAD design formats.

- Research Question - How to provide a data model which supports multiscale analysis integration based upon level of detail information within design formats.

- Research Question - Investigate whether it is possible to provide default versions of analyses, with different levels of detail and localisation, which apply to concept designs and then to more detailed designs as they are developed.

- Research Question - How can practitioners be supported in dealing with a dramatic increase in the quantity, complexity and level of detail in analysis results?

- Support Diversity \& Enable Integration - of analyses, of disciplines, of design data, of data formats, of to enable flexibility within the industry and allow collaboration while avoiding the fragmentation currently inherent. This will 
enable multi-disciplinary work by exploring the interactions of different models; as will supporting ad-hoc models which are common amongst practitioners.

- Engineering Issue - Wherever possible use standard techniques and data models which are common across the plethora of propriety and open data formats and industry standards. $\quad$ (such as those outlined in section 2.8 (p71))

- Research Question - Investigate format transformation engines and design query languages for translating between open standards.

- Research Question - Which methods of model integration are effective in this industry? For example shared ontologies, format transformation engines and automatic integration of models based upon ontologies.

- Integration of Sensor Data - from the physical design environment and existing designs into analysis models to impact design decisions based on existing conditions and current building performance. Sensor data could also be used to improve analysis accuracy by validating models.

- Research Question - Explore the most efficient means of collecting, communicating, storing and analysing sensor data in the built environment.

- Research Question - Explore algorithms for monitoring sensor data streams and producing insight.

- Research Question - Explore how sensor data can be integrated into existing insightful analysis models, for example building energy models.

- Improve Communication - provide tools for producing deliverables which enable better communication between disciplines, designers, clients, regulatory authorities and the general public. Through clear information visualisation and interactive $3 \mathrm{~d}$ environments.

- Engineering Issue - Provide clear, integrated and automatic 3d visualisation of quantitative data geolocated in an explorable environment.

- Research Question - What are the best methods for clear communication of complex engineering data in such a way that trends and anomalies are clear and provide insight across disciplines.

- Engineering Issue - How to provide online distribution of $3 \mathrm{~d}$ environments enabling communication of design and analysis results with the wider general public [94].

- Support Investigations \& Design Space Exploration - Provide practitioners with the capabilities to explore the design options and decision using analysis 
of multiple design and scenarios. Use techniques such as parameter studies and sensitivity analysis to rapidly explore the design space to provide insight for practitioners.

- Engineering Issue - Manage design and analysis data and allow efficient design space exploration to enable comparison and identification of trends across results.

- Research Question - How can a computational framework be flexible enough to support the rapid definition and composition of scenarios to define investigations into the design space.

- Research Question - How effective are design of experiments, optimisation and sensitivity analysis techniques in the context of the AEC industry?

In summary the context and end goal of all of these engineering issues and the research questions that drive them is to Produce Design Insight for the practitioners within this industry. This is perhaps the most critical concern since without producing design insight for practitioners and clients there is little utility for the adoption of the computational techniques proposed in this thesis.

\subsubsection{Focus of this Thesis}

Having a presented a broad range of computational research challenges within this industry we now focus upon the following research areas as the scope of this thesis. These research questions form the minimal set of progress required to demonstrate the benefits that a unified approach to computation could bring to this idnustry.

- Research Question - How can workflow automation platforms be adapted or created to target specifically the urban masterplanning community to improve integration between the design and analysis cycles with a faster analysis cycle.

- Research Question - How to provide a data model which supports multi-scale analysis integration based upon level of detail information within design formats.

- Research Question - How can a computational framework be flexible enough to support the rapid definition and composition of scenarios to define investigations into the design space.

- Research Question - How effective are design of experiments, optimisation and sensitivity analysis techniques in the context of the AEC industry?

In answering these questions their attendant engineering issues must also be addressed. The remaining research questions identified form the future work and hinge upon successful solutions to these questions. 
We will now explore the current state of the art in addressing these questions, starting first with lessons which may be learned from other more computationally unified industries.

\subsection{Product Optimisation Frameworks}

Computational automation in the aerospace, automotive and product design industries is substantially more advanced than current practice within the AEC industry [35]. Hence we consider first a number of integration and automation systems in use within these communities. We seek to identify the key computational techniques in use so that their applicability to the AEC industry may be evaluated.

\subsubsection{Comsol Multiphysics}

Comsol Multiphysics [27] is a multi-disciplinary design and simulation framework. It provides either import or live-linking with several mechanical CAD programs such as SolidWorks and enables the running of multiple engineering analyses including acoustics, computational fluid dynamics, heat transfer and earth science simulations. Hence a design can be analysed by multiple disciplines using a single framework. The results are visualised using a variety of two and three dimensional plots as well as visualisation in $3 \mathrm{~d}$ by texturing design geometry or displaying vector fields surrounding the component to demonstrate wind flow.

The framework centers on a "Model Tree". This holds all project data such as global definitions (design parameters), a geometry tree including geometrical elements and modifiers, a set of analysis models from various disciplines and finally a set of results. Each of these data sets is stored in a distinct branch of the "Model Tree". Various tree nodes may also contain child parameter nodes for configuring analyses. It is also common to link analysis nodes to geometry and variables. This allows linking analysis models to specific parts of the geometry of the design. For example linking a heat-flow analysis to a piece of geometry which is the heat-source.

Finally we should mention that Comsol Multiphysics has a strong focus on design optimisation. This is achieved by writing custom Matlab [68] plugins for the purpose which take advantage of Matlab optimisation routines.

Key capabilities, data models and computational techniques:

- Plugin system for analyses supporting a range of multidisciplinary analyses.

- Global parameter definitions.

- Single "Model Tree" holds all project data with subsections for design parameters, models, design and results. 
- Geometry tree breaks design down per geometry element for analysis.

- Design space exploration via optimisation, parameter sweeps and sensitivity analysis.

- Link analyses to parts of the geometry tree.

\subsubsection{Model Center}

Phoenix Integration's ModelCenter [80] is a simulation control and design optimisation package focused on the engineering and electronics industries. It provides strong links with CAD and engineering analysis programs such as CFD models. This enables the design of a simulation through a graph based layout allowing interconnection of CAD and analysis programs. For example connecting a parametric CAD program with a CFD package to try to optimise the design of a propeller. This data model places the tools as first class citizens rather than the design under analysis. By this we mean that the primary graph data structure that forms the user-interface consists not of design elements but instead of analyses and optimisation nodes. The strengths of ModelCenter lies in its strong integration capacities as well as the design space exploration and optimisation tools which are included. These include various design of experiments techniques for sensitivity analysis [71] and several optimisation approaches including gradient based solvers as well as genetic algorithms for less well formed design spaces. The focus on integration extends to the ability to connect to custom tools via a command line wrapper and a developers SDK for writing plugins via the Common Object Model (COM) interface. Phoenix Integration also provide tools to manage the running of design simulations on a compute cluster and collating the results. A wide suit of visualisation tools are also included to display response surfaces as the design space is explored.

The focus of this product is on the aerospace and automotive industries and there are currently no tools for connecting the types of analysis which are used within the architectural world. Though as described in section 2.5.2 (p58) explorations have been made to make these connections.

Key capabilities, data models and computational techniques:

- PHX CenterLink [79] enables grid computing to provide rapid computational analysis.

- Customisable analysis integration tools, wrappers and SDK.

- Focus on analyses and their links as first class citizens.

- Wide range of sensitivity analysis algorithms included to explore performance sensitivity to design parameters. 


\subsubsection{ModeFrontier}

Esteco's ModeFrontier [34] is an integration platform focusing on numerical multiobjective optimisation. It contains a wide range of plugins to link with analysis software and support a variety of scripting plugins for data processing and optimisation. Similar to ModelCenter the data model consists of analyses linked together rather than a description of the geometry as with the Comsol platform. One difference however is that experiment set up is included as a module within the workflow graph.

The platform supports design of experiments techniques [71] to enable design space exploration. This is a branch of statistics relating to constructing optimal sets of experimental runs in order to gain the most information about the design space. This technique is coupled with response surface modelling algorithms which create $3 \mathrm{~d}$ plots of design performance through a design sub-space. The platform integrates a set of multi-criteria decision making tools to aid decision makers in complex choices between alternatives. Again this software is focused on product design and the automotive industry with limited connection to the AEC industry.

Key capabilities, data models and computational techniques:

- Scripting plugins in a variety of languages

- Multi-criteria decision making tools

- Response surface modelling based upon design of experiments (sensitivity analysis)

\subsubsection{Isight}

Isight [29] is another product design workflow system allowing the integration of various analyses with optimisation algorithms and custom scripts. It is targeted strongly toward product design with integration to parametric design software such as Catia and focus on connection with engineering analyses such as finite element analysis. The software is able to explore the design space using design of experiments techniques, optimisation and Monte Carlo simulation.

Key capabilities, data models and computational techniques:

- Close integration with $3 \mathrm{~d}$ s product suite for product design

- Grid compute engine

- Design of Experiments analyses and optimisation engine. 


\subsubsection{Conclusions}

There are many widely used and well developed design optimisation / workflow systems for product design in the aerospace and automotive industries. These tools address many of the same challenges faced in the AEC industry, namely increasing the speed of analysis iteration, managing data and trying to address interoperability challenges.

However despite providing integration with a couple of cross disciplinary tools, such as AutoCad and support for computational fluid dynamics, there is very limited explicit support for crossing over to the AEC industry (with the exception of a study with ModelCenter which is described in section 2.5.2 (p58)). The missing links include integration with architectural design tools, particularly parametric tools such as Grasshopper and Rhinoceros. Similarly there are no links to the plethora of discipline specific tools such as IES for building energy simulation, nor to tools for traffic modelling, pedestrian modelling, fire simulation, infrastructure planning and similar disciplines.

Aside from this lack of model support one of the key reasons for the lack of adoption of these tools within the AEC industry is primarily a focus on optimisation not shared by architects. There are also organisational and skill set related reasons for not adopting these disruptive tools. None of these frameworks are targeted at the AEC industry specifically with custom integration to commonly used design and analysis packages. Finally the lack of an appropriate data model which centres on the design and its structure is also a barrier.

Whilst many of these frameworks focus on optimisation, this is not normally a key requirement in the AEC industry where analysis of the architect's design rather than the optimisation of the design is the key service being performed. That is not to say there is not scope and research into optimisation of design, however it is only a small proportion of current practice. Closely linked, within these frameworks, with optimisation are design space exploration techniques which are widely and successfully used to enable design insight by identifying the effects of varying design parameters. Foremost among these are various Design of Experiments (DoE) techniques which enable sensitivity analysis (see section 2.7.2 (p68)). Sensitivity analysis will identify the key parameters for affecting a Key Performance Indicator (KPI) enabling practitioners to focus on design improvement. This should be one key technique to enable the exploration of a large and unstructured design space found in architectural design and its efficacy in the AEC industry should be explored.

Another potential reason for a lack of transferability to the AEC community is the data model in use within these frameworks. There are a variety of data models in use, however all share a focus on letting the user to interact with the analyses and optimisation algorithms being linked together to form a workflow rather than on the design under study. This limits interaction with the design, its form and components. 
This is because all of these frameworks focus on whole product analysis rather than the analysis of design components (e.g. many buildings in an urban development). Only one of these framework (Comsol) explicitly includes the design and its components at a high level in the data model giving equal prominence to the design geometry and the analyses being undertaken. In part for this reason there appears to be no explicit support for multi-scale analysis. A representation of the design enabling multi-scale analysis is likely to be key for gaining support in the masterplanning community and should be explored in more detail.

We also note that model integration remains a key challenge in product design as in the AEC industry. Two approaches are taken: firstly the development of comprehensive plugins, and secondly support for ad-hoc integration via wrappers and python scripting. Within the AEC community there are a number of integration formats and frameworks which will be discussed in section 2.8 (p71).

Other key techniques include the parallelisation of workflow tasks and it is a common aspiration to export simulations to grid or cloud computing resources for analysis. Management of analysis results is another key enabler for these products as is a wide variety of visualisations of analysis results. These take either the form of $3 \mathrm{~d}$ environments showing physical phenomena on a $3 \mathrm{~d}$ model of the product being analysed. Or, alternatively a variety of design space, response surface and pareto front ${ }^{1}$ visualisations are used to communicate the undertaken design space exploration. These allow comparison of analysed design options on multiple axes or based on performance on an objective function, finally response surface modelling allows estimation of the performance design options not considered which lie between analysed alternatives. These techniques should be transferable to the AEC community with pareto fronts frequently used in computational design optimisation work as discussed in section 2.5 (p56).

Finally there are a number of systems modelling frameworks (e.g. [69]) which aim to simulate the performance of mechanical, electrical and hydraulic systems by creating intricate process and component diagrams of the design. However these are outside of the scope of this literature survey, since they are modelling environments for creating analyses rather than workflow environments for managing the multi-disciplinary analysis of a design understudy.

This thesis seeks to identify the transferability and efficacy of the techniques employed by these frameworks to the realm of architectural analysis on the urban masterplanning scale. We will now consider current research projects in this sphere.

\footnotetext{
${ }^{1} \mathrm{~A}$ pareto front is the set of designs which are pareto optimal. A design is pareto optimal only when there does not exist a design which performs better than the design in all performance aspects considered. A pareto front is normally shown visually on a 2 or 3 dimensional graph.
} 


\subsection{Architectural Optimisation Frameworks}

In this section we consider a number of research projects and commercial tools which are specifically targeted at the AEC industry. These tools have as their goal the optimisation of the design and/or the multi-disciplinary analysis of a design. We aim to explore their construction, data model and the computational techniques they apply along with their efficacy.

\subsubsection{Intelligent Design Objects}

"Intelligent Design Objects" [17] [25] is the title of the thesis work of Michael Bittermann. The objective of this work is to provide a means of integrated design analysis and optimisation on the building scale taking into account the qualitative aspects of design wherever possible.

The system is based around a parametrised design and a genetic optimisation algorithm. Design analysis is undertaken by a novel probabilistic visual perception model which simulates human perception of objects, for example the visibility of a lift from a building entrance. The measurements taken by this means of analysis along with certain spatial measurements are fed into a performance tree which evaluates the design. Design performance is first standardised using expert-defined preference functions into the range $0-1$. Individual preference measures are then aggregated via a neural "fuzzy" tree which uses Gaussian functions and user weightings to create an aggregate preference metric. This method of design assessment is then used with a genetic algorithm to optimise the design. A relaxed Pareto front method is used to produce clusters of near optimal designs. The first set of child nodes in the performance tree are used as the aspects of the design to optimise for.

This automatic "Intelligent Design Objects" optimisation system is envisioned to be used by the designer as part of a bigger design loop. Successive rounds of optimisation produce groups of Pareto optimal designs, comparing between these allows the designer to refine their design and their design criteria encoded in the performance tree. The IDO system has been applied to two published case studies. First optimising the layout of a foyer to maximise retail space and resolve several navigation concerns and secondly optimising the arrangement of a small group of houses to maximise both garden space and visual privacy. In both cases a range of novel solutions were produced and optimised.

The performance tree within the IDO system is effective and provides the designer with a breakdown of preference. However this means that although multiple areas of preference can be considered, the raw quantitative performance figures are not displayed to the designer (being obfuscated by the preference functions). This limits the designer's knowledge of the design, reduces analysis and limits the scope for clear 
decision-making since decisions are made on subjective measures of preference. In part this is due to the type of an analysis employed (a visual perception engine). Additionally the performance tree undertakes an aspect-orientated ${ }^{2}$ performance decomposition - that is each sub-tree covers a different performance criterion or aspect of the design. In this context, aspects are simply different axes for assessing the performance of a design. This aspect-orientated tree is good for enabling the designer to express the goals the optimisation system should meet, however it does not necessarily support analysis of design performance objectively since the performance of design components is not mapped. This aspect orientated performance decomposition is in contrast to a component-orientated one where the components of the design are displayed in the tree and generate performance (see for example section 2.5.3 (p58)). This means that it is difficult to pin down each component's contribution to the design's performance so as to help the designer envisage the most effective areas of the design.

The IDO system is clearly a novel and successful design optimisation system, producing interesting optimised results in case study applications by using the novel visual perception analysis. However more widespread application of the framework is difficult since it does not have a (published) underlying computational framework for integrating other analyses beyond the one developed in the thesis. This limits the effectiveness of the system since it cannot support analysis from multiple disciplines so as to give a wider overview of design performance. The performance tree with its preference capture and processing appears effective providing it is set up correctly (no information is given on how long this process takes). The optimisation algorithms are effective at optimisation however while the designer can explore optimal design the system gives little information on the effect of particular parameters or decisions.

Key capabilities, data models and computational techniques:

- Integration with parametric design tools.

- A novel probabilistic visual perception model which simulates human perception.

- Genetic optimisation algorithms.

- Relaxed Pareto optimality criteria to generate a range of near optimal designs.

- Performance tree measuring preference with neural "fuzzy" aggregation functions.

- Aspect orientated performance tree.

\footnotetext{
2 "aspect-orientated" in this work refers to the evaluation of design performance and should be differentiated from aspect-orientated programming which is a different concept.
} 


\subsubsection{Multidisciplinary Process Integration and Design Optimization}

In their paper "Multidisciplinary Process Integration and Design Optimization of a Classroom Building" [36] Flager et al discuss and demonstrate the application of Process Integration and Design Optimization (PIDO) techniques common in the aerospace and automotive industries to an architectural design study. To do this they exploit Phoenix Integration's ModelCenter (see Section 2.4.2 (p52)) to carry out integration and optimisation tasks. The resulting system uses a parametric CAD model connected to a number of different evaluation packages such as a Finite Element Analysis (FEA) package used to test structural integrity, and a construction cost model. This system is then optimised to try to create a design of maximal performance. The results are encouraging. The process is able to optimise a design successfully on aspects calculated by different analysis packages while considering a number of constraints. The project also overcomes several integration problems though not without several hundred hours of development time.

Whilst this system is effective at optimising the case study design it allows only parametric variation of a small number (6) of design parameters, which does not constitute a large fraction of the total design space. Analysis results similarly cover the whole design and do not give insight into the performance of particular design components. Similarly there is no insight into the composition of the design's performance which might be given by use of a performance tree. Whilst Model Center's design space visualisation techniques are effective there is no integrated visualisation of the actual design under consideration. However this approach does show the potential benefits of adopting techniques from product optimisation systems to the AEC industry; particularly from design space exploration and a standardised integration framework.

Key capabilities, data models and computational techniques:

- Used Model Center platform for integration and optimisation providing standard interoperability and integration.

- Genetic optimisation algorithm

- Integrated multiple design analysis programs

- Design of Experiments techniques for design space exploration

\subsubsection{Component-oriented Decomposition for Multidisciplinary Design Optimization}

In [38] Philipp Geyer presents a "Component-oriented decomposition for multidisciplinary design optimization in building design". This design optimisation system works by optimising building components. This system utilises annotated Industry Foundation Classes (IFC's - see section 2.8.5 (p74)) to represent design components (such as 
rooms, roofs and steel frames) and construct a model of a building. This is then annotated with analysis layers from multiple disciplines to compute performance (e.g. cost of a steel frame element). This is aggregated by a separate performance tree which aggregates per-discipline performance trees together with a preference tree using preference functions. This system is then used with a multi-objective genetic algorithm to optimise a building design to meet the specified requirements of an architect. Different choices of material, construction and dimensioning are enabled by varying the IFC classes involved in the design. Structural analysis ensures the design is realistic and some components contain an optimiser to ensure correct dimensioning. Other analyses test performance for different disciplines. Integration with Phoenix Integration's Model Center (see section 2.4.2 (p52)) enables use of a multi-objective genetic algorithm to optimise the design to meet numerous goals simultaneously. The use of this system is envisaged similarly to the Intelligent Design Objects system described in section 2.5.1 (p56) with the designer setting up the model, running the optimiser then reviewing the results and modifying the model and performance tree to suite new design features and objectives. The optimiser will not only try to meet the constraints extracted from the components but also to choose the best types of materials or types of structure (e.g. beam type) in order to meet the specified goals. This together with the realistic engineering, involvement of multiple disciplines and intelligent components are used to produce realistic designs and are the key and novel features of this work.

This project makes good use of IFC's for design representation as a key enabling technology for creating the design decomposition which forms the heart of this project. However questions remain over the complexity of creating such intricate building, analysis, evaluation and optimisation models. Particularly it would be interesting to consider the fragility of the highly-interrelated design components and their analysis annotation$\mathrm{s} /$ links and links between the performance tree and the analysis results. It is interesting to see separate performance and preference concerns in the design evaluation tree since this will enable quantitative assessment of design performance, however similar to [25] the modelling of accurate preference representation is challenging.

The initial set up of IFC's can constrain the design space exploration that is possible [45]. For example by not allowing variation in parts of the component graph or by shaping the graph to only accept particular structural solutions (e.g. cuboid shaped buildings). This is something the author has addressed in other work [39]. However this system remains more expressive than the IDO system discussed in section 2.5.1 (p56), as it enables the use of different components (e.g. different steel structure types) rather than just the dimensioning and parametrisation of objects seen in the IDO system. It would be interesting to consider how this approach could be scaled to the urban masterplanning level, perhaps by taking advantage of city-level interoperability standards such as CityGML (which will be discussed in section 2.8.2 (p72)). Finally 
since a component-orientated decomposition is taken it would be interesting to consider per-component performance, however this is not discussed within the project. How the concepts and techniques in this project map to the urban masterplanning scale will be discussed during this thesis in section 3.8 (p108).

Key capabilities, data models and computational techniques:

- Use of Industry Foundation Classes for design representation

- Annotation of design elements with analyses

- Component-oriented decomposition of the design for detailed analysis

- Performance tree containing per discipline subtrees together with a preference tree

- Multi-objective genetic algorithm

- Design variation via design component change and dimensioning through the IFC standard [22]

\subsubsection{Conclusions}

These architectural optimisation systems are part of a larger field of research into design optimisation. This has been driven by twin goals of:

- Optimising specific designs beyond the capability of human designers (e.g. minimising cost of steel work [102] [96]).

- Improving overall design beyond or without an architect's vision.

- Producing new innovative design forms.

This is enabled by increasing computational power and more recently by parametric and procedural design tools. The systems discussed in this section show the potential for genetic optimisation algorithms as a key enabling technique for searching the varied and unstructured design spaces frequently found within architectural design. Whilst such systems are proving effective at refining a design and indeed are being adopted more widely (e.g. [89]). There remains limited adoption of optimisation systems to refine designs. It is extremely uncommon to use optimisation as a primary design tool. Thus these research frameworks find limited adoption and a wider research question remains of finding the appropriate computational support for design and analysis in the AEC industry and specifically in the realm of urban masterplanning.

In terms of computational techniques which have been applied in these projects, it is clear that design space exploration has great scope to produce design insight. Similarly the analysis automation this requires is also an imperative. However as evidenced in 
[80] analysis automation and integration remain a challenge. The use of design decomposition in [38] while primarily providing the opportunity to explore design alternatives by changing design components may also provide a simple and effective way to explore design performance. Similarly annotating design elements with simple analyses is an interesting and effective way of assessing performance at different scales.

Much of the research into design optimisation and many of the frameworks explored here lack an explicit underlying computational framework for handling design data, analysis set up and analysis data. The exceptions are the use of the Model Center integration platform for connecting analyses in [36] and the use of IFCs in [38] which are used to store design and are annotated with some analysis information. However while the use of Model Center [80] was effective for optimisation, it disconnects the design from the user since they can only interact with the analysis setup. Hence the use of IFCs for storing the design is attractive, however these are not commonly used at the scale of urban masterplanning nor at concept design. Thus an effective computational framework should allow design decomposition, ideally using industry standards if available and then allow annotation of these design components with analyses to be run.

Several of these systems utilised a performance tree which appears as a critical technique (e.g. within [17] [38]) for assessing design performance within design optimisation. However different forms, construction and focus are frequent. Example variations in the design of performance trees include:

- Consisting of preference or quantitative representation of design performance.

- Per-aspect or per-discipline breakdown of performance.

- Use of preference functions.

- Method of aggregation.

Guidance on the best form of tree is difficult to find and tree construction remains subjective. One missing method of constructing a performance tree seems to be considering performance per component, reflecting the design decomposition. This should be explored further. One concern with using performance trees is the difficulty of assessing the correct weightings and preference functions which must be "expertly" defined. Whilst there are processes for defining these [86] this remains a potential risk for their adoption. So too does a different intended use within the urban masterplanning community. Specifically instead of using performance trees as a tool for assessment of designs within an optimisation engine, using them for giving insight to a human architect will likely necessitate different construction, specification and use of performance trees to enable focus upon aspects of the design most important to practitioners. This will require further investigation. 
Finally we see that within this research, design space exploration via optimisation is seen as the key technique. However no emphasis is placed on non-optimisation led exploration. For example using design of experiments and sensitivity analysis. This is in contrast to the product optimisation frameworks (see section 2.4 (p51)) where such techniques have more prominence. Early exploration from [36] reports promising results and this should be explored further; particularly given the complexity of the analysis models and design space within the AEC industry.

Finally we note that using optimisation loses out on the insight of design and analysis practitioners insight and experience. Particularly as much of this insight, experience and intuition and is not codified. Optimisation tools in part aim to resolve the gap between the design and analysis communities by automating the design cycle into the analysis cycle. We will now discuss the opposite attempt.

\subsection{Integrated Design Analysis Packages}

The projects discussed in the previous sections have attempted to bridge the gap between the design and analysis cycles by incorporating the design process into the analysis cycle as part of an optimisation cycle. On the other hand there have been opposite attempts to bring the analysis process directly into the design cycle. This has been attempted by incorporating analysis tools directly into the design environment. We will now discuss a number of these projects, most of which are commercial in nature.

\subsubsection{Autodesk Suite}

Autodesk is one of the largest makers of software for the 3d design and architectural industries. It has a strong focus on design tools such as Revit [11] and Autocad [5], however recently Autodesk have released a series of products and projects which aim to integrate analysis tools into their design environment itself. The driver of this change is increasing concern over the performance of buildings from a sustainability and carbon perspective. We will now consider three projects driven by this goal.

\section{Ecotect}

Autodesk Ecotect [6] is a design tool which includes basic analyses for quantifying the visual impact of a building, its energy consumption, estimated cost, water consumption and solar radiation performance [7]. This tool aims to bring integrated design, analysis and visualisation together in one environment while making the analyses simple enough for a non-discipline experts to use. The system is backed by integration with Autodesk's Building Information Management model software to provide a "concept-to-detail" life cycle. The tool is backed by Autodesk's green building xml interchange format [37] 
to allow interoperability with other products. However this requires other Autodesk programs and environments and different drafting skills.

\section{Green Building Studio}

AutoDesk's Green Building Studio [8] [9] is a web-based service for analysing a CAD model of a building on multiple sustainability axes. With a focus on early stage design analysis it integrates into AutoDesk's CAD products via a green building xml interchange format [37] for exchanging coarse building designs. The web-interface then allows the designers to set up a number of analyses to be performed. These include energy and carbon analysis, water usage and daylight factor analysis as well as potential reports for renewable energy technologies such as photovoltaic and wind. Results are returned either in report form or in a web-based viewer. The system allows comparison of design alternatives via manual set-up of different scenarios. Changes in building orientation, materials and energy systems are allowed however design changes are supported only by importing new projects.

\section{Project Vasari}

Autodesk's Project Vasari [10] is a an integrated design and assessment suite for use on the neighbourhood scale. It uses parametric design tools together with design assessment tools similar to those found in Ecotect. This combination enables the designer to more quickly explore the design space and create more complex forms. The integration of realistic physics into the environment similarly advances this concept. The environment includes a facility to create and compare different design options. The project also focuses upon the neighbourhood scale and as such could be used for early stage masterplanning.

\section{Conclusions}

These three closely related design and analysis systems do a good job of generating design insight that helps to create a more sustainable built environment. The addition of quantitative analysis at an early design stage is critical since it is the time when there is most scope for improvement (or otherwise!) in design performance. The rapid quantitative design assessment these tools offer from the perspective of many disciplines is key in providing this design insight. Similarly the integrated visualisation showing analysis results clearly in context within the CAD environment is another key technique since it is a medium the designer will comprehend rapidly.

On the other hand this suite of tools is limited in a number of ways. Most notable is the lack of an open framework underpinning the integration of analyses. Similarly the analyses used are all high-level analyses with limited option to include more complex 
detailed analysis as the design develops (although export of Energy Plus [101] configuration files for further analysis is possible). Nor is there detailed control over the set up of the analyses. Underlying these concerns is the loss of insight from engineering practitioners in the same way that automating the design cycle looses the insight of the designers.

Whilst these suites do provide the ability to compare design options and projects there is are capacities for exploring the design space systematically. This would enable the more quantitative assessment of the design space enabling identification of optimal configurations for certain parameters. Finally there is no capacity within these systems to integrate analyses together which is a technique used to good effect in the previously considered optimisation packages. Similarly once the design becomes sufficiently detailed there is a need to move to more advanced and complex design and analysis packages.

Across these projects key features and computational techniques include:

- Parametric design tools

- Integrated design and analysis

- Integrated visualisation of design and analysis results

- Rapid feedback on designs

- Closely integrated design and analysis cycles

- Cloud based analysis service

- Integration with product suite via gbXML standard

- High level analyses which work at concept design scale

- Parametric design tools for simple design modification

- Ability to compare multiple design options

\subsubsection{CityCad}

Holistic City Software's CityCAD environment [49] is a CAD environment targeted specifically at the urban masterplanning community. It uses a parametric city modelling environment with object classes specifically targeted to masterplan design (roads, pavements, parks and so forth). This object library enables rapid quantity surveying to produce accurate landuse schedules, generalised costs and parking requirements. The system integrates simple models for light simulation and estimating quantitative performance aspects of the concept design. These include estimated onsite energy generation and consumption as well as water consumption and waste generation. Other novel 
assessments are made for identifying what percentage of the development is within 5 minutes walk of a bus stop and what percentage of the road network is too steep for accessible pedestrian access. Many of these analyses can be visualised within the design environment and visualisation exported to clients. This approach is similar to the (now defunct) PixelActive3d [48] city design tools which focused upon integrated design and visualisation using parametric techniques for road and geometry generation. However CityCAD includes many more analyses which are relevant to the design team.

Once again this highly integrated design and analysis suite shows great potential in enabling quantified design improvement. However again we see a closed framework which shuts analysis engineers out of the design assessment loop at the critical early concept stage of design.

Across these projects key features and computational techniques include:

- Quantification of design through well-designed geometry meta data.

- Integrated design and analysis through geometry types.

- Integrated visualisation and design.

- Integrated novel high-level analyses appropriate for urban masterplanning concept design.

- Rapid design assessment.

- Brings quantification to the design process since all design objects are typed and dimensioned.

\subsubsection{Conclusions}

This counter trend to computational design optimisation has also shown the great potential for unifying computational design and computational analysis. The surprising effectiveness of the seemingly trivial analysis and quantitative measurement integrated into some of these tools (e.g. "number of parking spaces required" in CityCad) is unexpected when compared to the complexity of the analysis tools normally applied to answer such questions. This is in part due to the high level concept design being undertaken where less information is available and a less accurate, simpler estimate is acceptable. Whilst these analyses are clearly limited and will not provide sufficient quality of insight throughout the design process they work because they enable rapid quantitative feedback. This is particularly important at an early concept stage since it gives greatest scope for design improvement before important decisions are fixed.

Whilst these tools are effective for the design community, particularly when detailed design is required, they are not able to support the full design process. This will frequently involve more complex design drafting tools and more advanced analyses run 
by engineers. The rework necessitated in moving between such design (and analysis) packages is a known challenge within the industry [32]. This is made more difficult in these cases since the software environments are not easily open to extension. This limits the breadth and indeed depth of analysis which may be undertaken. Similarly these tools do not support the coupling of analyses for greater insight. Finally while some of these tools support manual exploration of the design space none of the tools we considered supported computational exploration of the design space nor manual scenario based exploration. These techniques which have been successfully applied in design optimisation could provide great value to the designers also.

In conclusion we see the same need for unification of the urban masterplanning design and analysis communities from the design perspective as from the analysis team. The unification of the best computational techniques from both communities will be paramount to successfully improving the design/analysis workflow and generating design insight for improving design performance.

\subsection{Techniques}

In this section we explore a number of computational techniques and models which have proven useful in generating insight for practitioners and addressing the challenges identified in section 2.3 (p47).

\subsubsection{Visualisation}

As an example of the power of multidisciplinary quantitative visualisation we consider Arup's 3d Urbanism research project [65]. For this project a single district from a larger urban masterplanning project was targeted for multidisciplinary analysis and integrated visualisation. The intended benefit was to show the power of visualisation for improving communication between disciplines, the urban masterplanning team and their client.

Fig. 2.1 (p67) is a screenshot from the final project deliverable showing numerous disciplines visualised together. Using the $3 \mathrm{~d}$ environment each discipline can be explored independently avoiding any visual confusion. Discipline analyses which were applied to this case study were:

- Daylight factor showing average day light over the course of a year based upon sun position and location of the development. This enables identification of dark alleyways, thermal heat gain issues and consideration of right-to-light issues caused by tall buildings.

- Wind flow under the prevailing wind conditions of the project location. This enables the designer to avoid urban forms which channel wind or snow violently 
at pedestrians.

- Traffic analysis showing traffic flow at peak and off peak times. This helps identify problematic street design and with acoustical analysis enables exploration of quality of life in residential developments.

- Acoustic analysis showing expected background noise from transportation. This enables exploration of the quality of space within courtyards of residential developments.

All analyses were computed manually offline by experts in each area then integrated manually into the Quest3d game engine [82]. Each analysis was based upon the same geometry and in some cases analysis used the output of others (for example acoustics based upon traffic flow). Overall the analysis and visualisation for this target area took some 6 months highlighting the problems of data interoperability and slow analysis cycles identified earlier in this chapter.

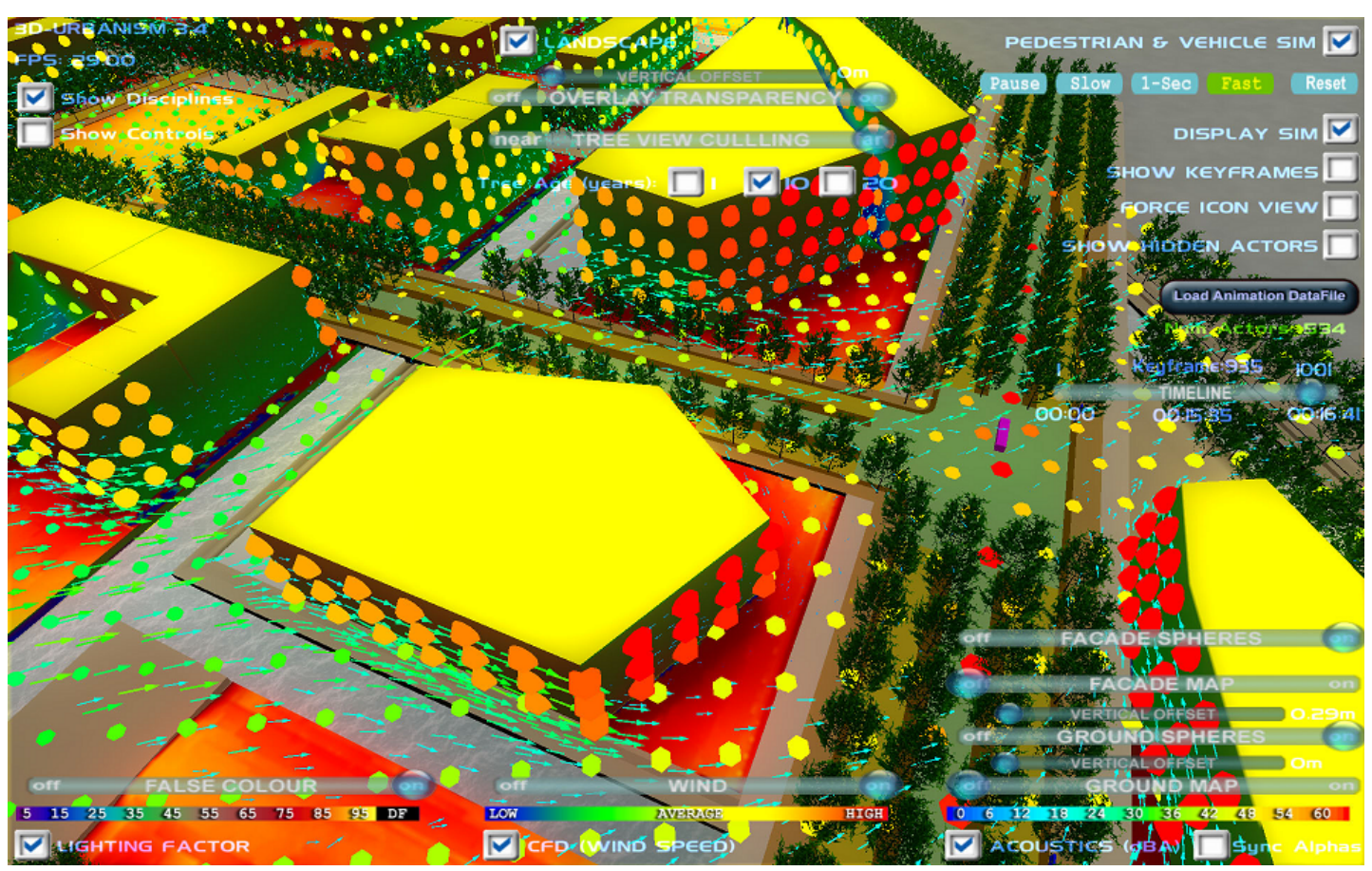

Figure 2.1: An example visualisation from Arup's 3dUrbanism project [65] integrating design analysis from many disciplines on the scale of a neighbourhood. Buildings are textured with a daylight factor map showing average annual daylight. A Computational Fluid Dynamics (CFD) analysis of the prevailing wind direction is shown by a vector field displayed as arrows. An acoustic analysis of the area is displayed as differently sized spheres. Finally a traffic simulation is shown as an animation representing expected traffic flow. Using the $3 \mathrm{~d}$ environment each discipline can be explored independently avoiding any visual confusion. 
The visualisation itself is useful for highlighting design challenges such as courtyards which are too dark or noisy, roads and problematic areas for windflow. The interactive environment is highly engaging and entices clients to explore the analysis results themselves, particularly when comparing two disciplines together such as traffic and acoustics and noticing their interactions. Another challenge was the length of time that preparing this visualisation took (some six months) most of which was consumed by lengthy analysis process. Additionally the scale of the analysis and visualisation is limited to only a portion of the urban masterplan. This is due to scalability limitations in the visualisation engine and some of the analyses. Despite these challenges in creating such a visualisation and perhaps a need for improved visualisation techniques this is clearly a powerful technique for aiding communication and gaining design insight.

\subsubsection{Sensitivity Analysis}

One technique which is used frequently in engineering product design is a sensitivity analysis. This technique can give insight into the design space by measuring the sensitivity of a KPI to changes in a range of inputs. The inputs that the KPI is most sensitive to will have the most scope for design improvement. Effectively a sensitivity analysis provides a map of the design space showing the most effective directions to travel in by changing particular design parameters.

A sensitivity analysis is set of experimental runs which vary the input parameters in a specific way in an attempt to understand the effects of each parameter on the outputs of the experiment. To construct such experiments a range of valid inputs must be set for each input (e.g. maximum value and minimum value) which are then used to create large sets of experiments. The design of such sets of experimental runs is a branch of pure mathematics called Design of Experiments (DoE) [71] and there are numerous designs which are more or less efficient in different scenarios and give more or less insight into the experiment.

Within the architectural field we can use sensitivity analysis to explore the design space and use the sensitivity results to show the designer which design parameters will have the biggest effect (are most sensitive) on design performance, enabling the designer to most effectively target their time. We should note that if we employ multidimensional sensitivity analysis we can look at a parameters effect on multiple aspects of the design, thus we can pick out the most effective design parameters to modify in order to achieve the set of effects being aimed for. The following are the use cases of sensitivity analysis in the AEC industry:

1. Designer Guidance - By this we mean that the sensitivity analysis can highlight the set of parameters which have the most scope for impact upon a given performance goal, allowing them to focus on improving the design in the most 
efficient manner possible.

2. Design space exploration - In conducting a sensitivity analysis we are constructing a set of parameterisations of the design. These runs cover the design space (and analysis space) to some degree and thus can be taken to give an overview of the design space.

3. Identification of the effects of assumptions - If analysis assumptions are included as inputs to the sensitivity analysis by setting a maximum and minimum value for the input reflecting the uncertainty of the assumption the sensitivity analysis can identify whether this uncertainty is important to the KPI by how sensitive the KPI is to changes in uncertainty.

\section{Plackett-Burman Designs}

As first reported in the paper by R. L. Plackett and J . P. Burman [81]. PB designs are one of the least expensive designs for a multifactorial experiment. They enable the creation of designs analysing $k$ inputs (or less) with $N$ experimental runs where $k=N-1, k>N$ and $N$ is a multiple of 4 . This linearity in the number of runs makes a PB design one of the most efficient to run. The construction of these efficient designs is due to the particular class of matrices in use and the interested reader is referred to [81]. However the cost for this efficiency is that the alias structure of the design becomes very hard to decipher, since the main effects are partially aliased (confounded) with their interactions. By this we mean that the effect of inputs $A$ and $B$ is confused with the impact of their interaction namely $A * B$. For example in a twelve run design each main effect is aliased with 45 two factor interactions. This can make the PB design dangerous to use, especially in cases where two or more factor interactions are likely to be significant. One means of improving upon the PB design is by using foldover to reduce the aliasing effects at the cost of doubling the number of runs involved. Folding over the design enables the main interactions to be extracted along with selected two factor interactions. The mechanics of how this happens depends upon the size of the design and in some cases makes the PB design less efficient in terms of information generated than applying a standard factorial design. The main usage of a PB design is when the number of parameters under consideration or the length of time for each experiment (analysis run) means that the primary consideration in design choice is computational efficiency. Given the complexity of architectural analyses this is very likely to be the case.

\subsubsection{Performance Trees}

Another technique which has been successfully applied in the realm of computational design optimisation [25] [38] is the performance tree. This is a means of combining many 
KPI's and aggregating them together to form a single representative measure of the performance of the design. Fig 2.2 (p70) shows a typical construction of a performance tree.

In such trees the inputs to performance trees are generally design parameters and analysis results (KPI's). The performance tree itself will normally contain one of two types of data. Either (normally) numeric performance figures such as kwh electricity consumed per annum or a measure of preference which is mapped into an arbitrary scale, normally between 0 and 1 . Preference attempts to represent the practitioners view of the design performance. There are many ways of assessing preference and a number of techniques have been proposed to try to ease this challenging and subjective feature [86]. Most commonly a set of preference functions which map from performance space to preference space are defined and used. At each level in the performance tree aggregations are made based on the child nodes values to produce a higher level description of performance. There are a large number techniques for aggregating preference and performance from simple weighted average to "fuzzy" neural trees [25]. Indeed, there is a wide variety in the form of a performance tree.

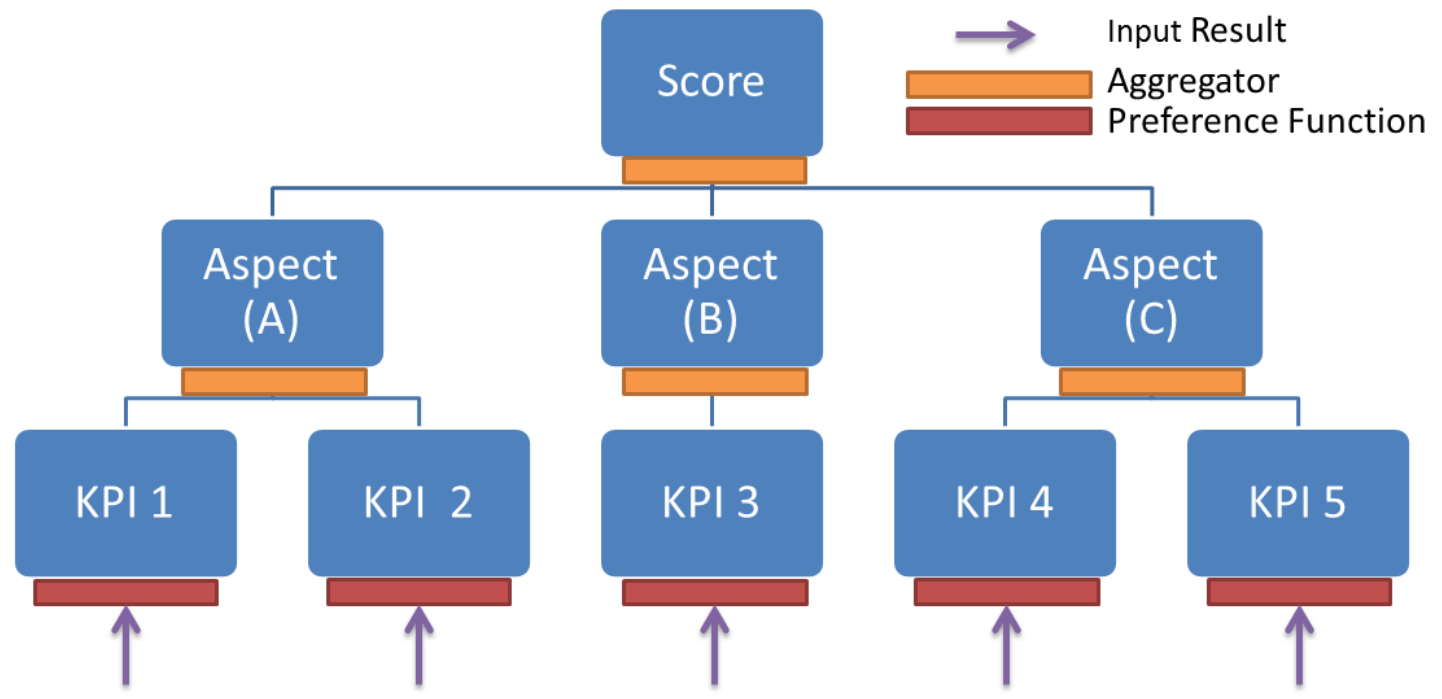

Figure 2.2: The structure of a generic performance tree, showing the use of preference and aggregation functions. Design parameters and analysis results are used as inputs to the leaf nodes of the tree. These are optionally transformed into preference space using preference functions. Working up the tree are KPI's from a single discipline or covering an aspect of design performance are then combined via aggregation functions. Finally at the root of the tree a single score is created through an aggregation function.

There are many opinions on how performance trees are best formed. For example either containing only preference, only performance or a mixture of both. Similarly whether the tree is constructed to break down performance or preference per design 
aspect or goal (cost, performance, size...), per KPI, per discipline or per design component. Indeed a mix of all of these options is possible and each will have its advantages and disadvantages and will often be down to the personal preference of the user; who's judgement the performance tree is trying to emulate. This shows the primary subjective challenge which is setting the weighting and structure within the tree, its aggregation, and its preference functions. Some tools exist for helping the setting of these weightings e.g. Analytical Hierarchical Process [86] however this remains a challenge.

Another advantage of using a performance tree is the rapid diagnosis of performance or lack thereof - for example a poor score given to a building can be traced down through the children of the root node to identify which discipline, aspect or KPI performed poorly. This can then be broken down again to identify the poorly-performing element or result more definitively; particularly if performance is visualised in some way it is easy to trace performance or preference down through the tree to identify root causes. Similarly comparing performance trees can be a valuable way of identifying differences between design alternatives and their performance. Examples of this are shown in Fig. 4.16 (p170).

Performance trees should be a key technique for aiding communication of quantitative results and preference within urban masterplanning. Particularly given the wide design space which is likely to be encountered, performance trees should prove a simple visual means of communicating performance of competing design options to practitioners and clients. This, however, is a different use in the AEC focused projects we have considered in this chapter [25] [38] and will need investigation.

\subsection{Integration Systems \& Formats}

Within the AEC industry there are a large number of integration systems and formats. These are not automation systems but help to avoid the interoperability challenges associated with urban masterplan analysis. In particular we explore data storage and interchange formats and systems. Some, such as DesignLink [3], consist of a library for transforming many file formats to and from a central storage format. On the other hand Industry Foundation Classes (IFC) [22] and CityGML [60] consist of a large welldefined format which it is intended will be supported industry-wide by all proprietary design and analysis formats. Finally there are information systems such as Geographic Information Systems (GIS) and Building Information Models (BIM) which can either be used as stand alone file formats or more commonly be used as a central data server enabling many users to interact with the data. Each of these systems has its strengths and weaknesses. They are used with various levels of uptake in different yet often overlapping areas of the urban masterplanning process. We will now discuss each in detail. 


\subsubsection{Collada}

The COLLAborative Design Activity (Collada) [55] format is curated by the industryled Khronos Group [56]. It is an open xml based format for 3d geometry and scenes. It is aimed at geometry interchange for $3 \mathrm{~d}$ modelling and the games industry. A wide range of tools in frequent use within the AEC industry support the Collada .dea format for interchange of geometry which helps to address interoperability challenges. However the format stores geometry interchange without associating the meta data needed for analysis with geometry elements. This means that while geometry can normally be transferred it lacks the required information for analyses such as identifying room volumes and knowing which buildings are of which building type. Whilst some steps toward providing meta data have been taken (v1.5 provides support for physics kinematics and material properties [57]) this format does not resolve interoperability challenges sufficiently to ease the interoperability challenges between the design and analysis communities. Since although design geometry can be transferred, significant rework is often required to add meta data and change geometry layouts.

\subsubsection{CityGML}

In a similar sphere to Collada the CityGML (Geography Markup Language) [42] [59] [60] format is an interoperability standard for describing 3d models of cities and is one of a family of geography interoperability standards. It includes topological features, roads, tunnels and buildings at several levels of detail. In contrast to Collada it contains sufficient semantic detail to enable some analyses to be undertaken on the city described, for example noise pollution models have been run using CityGML data sets [28]. The xml based standard supports five levels of detail from a regional model through to a city with explicit roof structure to an interior model of buildings. The format contains separate trees for storing the semantic model of the city (city, buildings, rooms) and the geometrical model (plan, cuboid, mesh) which are linked together explicitly.

This format has the potential to bridge many of the interoperability gaps between urban masterplanning design and analysis particularly at the early stages of design. This is due to support for several levels of detail which can be filled as the design develops. Similarly the annotation of design elements with meta data allows various analyses to run, for example extracting a breakdown of development by dwelling type. However unfortunately the CityGML format is not widely used within industry, and particularly is not used widely within the design tools used by the architectural community. However other projects with a similar approach, including Green Building XML and the Industry Foundation Classes standard, are gaining greater adoption as part of a drive toward Building Information Management models. 


\subsubsection{Green Building XML}

Green Building XML (gbXML) [37] is a format for exchanging building level designs amongst design packages and analysis models from a set of providers. The format is focused upon concept and semi-detailed design with a view to energy analysis. The format may contain multiple buildings however it does not cover the same range of building types as CityGML (e.g. bridges and tunnels). The format is widely supported amongst commercial CAD and analysis packages. The format supports storing detailed analysis results such as the sizing of a heating and ventilation system. Support for building energy analyses is one of the primary uses of the format. However the format is not sufficiently broad to support all aspects of the masterplanning geometry nor analyses used on this scale. Extending these formats with greater scale and detail would prove challenging and given the size of other formats (e.g. IFCs) which are still expanding despite containing over 700 entities

\subsubsection{BIM}

Building Information Management [31] or BIM is a wide term covering a spectrum of standards, processes, approaches and methodologies which relate to building scale, design, analysis, construction and operation. BIM generally refers to highly annotated detailed digital $3 \mathrm{~d}$ designs of buildings and their subsystems. This brings many benefits through the coordination of complex drafting, design and analysis across the AEC industry. There are a number of BIM interoperability standards such as Industry Foundation Classes which will be discussed in the next section. Frequently there are BIM servers such as Revit [11] which act as repositories for large BIM designs and allow multiple users to edit a design. BIM is generally used only at the more detailed stages of design and generally only on the building scale. It's use at the early stages of design and at urban masterplanning scale are rare. In part this is due to the complexity of the standards and finding them either too broad (requiring too much information) or too narrow (not covering all aspects of the design elements required for early stage design or all disciplines involved). This will likely continue to be a challenge with early stage design and is a ripe area for research. Particularly since the use of BIM will be mandated by UK government on all infrastructure projects by 2016 [99]. Hence BIM is very much the future of the industry, however research is still required on the best computational techniques and platforms to make use of BIM standards and facilities. For example platforms for automating analyses and design spaces using BIM techniques are rare. Finally we note that BIM approaches have been seen more as a replacement for digital drafting than as a design and analysis tool. 


\subsubsection{IFC}

Industry Foundation Classes [22] are the primary interoperability standard within Building Information Management (BIM) models. The standard is developed by the buildingSMART international industry consortium [21]. The standards are now ISO ratified as ISO/PAS 16739. The suite of standards are all xml based and take an objectorientated view of the designs and their components. The standard aims to cover many of the disciplines which are involved in the AEC industry. Indeed there are some 700 foundational classes covering entities from rooms to structural beams each of which will contain a number of attributes providing detailed information about the entity. IFC classes are frequently nested to provide complex hierarchies of objects, however the standards for these hierarchies is contentious [45].

Alongside the IFC standard various complimentary standards have developed such as Information Delivery Manual (IDM) [24]. This aims to help with construction phasing by specifying when certain components are required for the building process. This is a "request for information" specification system for use during construction or operation. To ensure coherency within and between standards an International Framework for Dictionaries (IFD) [23] has been developed to provide a common ontology for IFC classes, attributes and product data allowing closer more rapid integration.

While the IFC classes are very broad, they are limited in that they will not, without substantial extension, be able to contain all of the analysis results from all of the disciplines encountered during masterplan design. For example they do not cover district energy systems nor transportation analyses. Similarly the standard is very broad for concept level design which does not contain detailed information; particularly as there are few design tools which will natively design using these standards. Hence the standard is normally used for data exchange between programs toward the detailed and construction phases of design. To address this challenge and to reduce information overload on practitioners Model View Definitions (MVD) [46] have been developed. These are essentially windows onto a BIM model which specify the required information for view by only one discipline or for export to another program.

In conclusion while the IFC ecosystem is a key enabling technology for a future BIM enabled world; it is not the solution to interoperability challenges within either high level concept design nor in urban masterplanning. While much success has been achieved in detailed design, construction and facilities management. A lack of support from design tools, a difficulty in covering the scale, scope and breadth of urban masterplanning mean that IFCs are not the complete solution to the challenges described in section 2.3 (p47). 


\subsubsection{DesignLink SDK}

Taking a different approach to integration we consider Arup's DesignLinkSDK [3]. Originally developed internally and now released under a collaboration license, the DesignLink SDK enables data transfer between several CAD programs and many analysis programs. The framework was "born out of a desire to integrate parametric-associative modelling software with analysis software, in such a way that the parametric model can be used to explore design options during the lifetime of a design project." [2]. Which is similar to the challenges identified earlier in this chapter. The framework revolves around a central evolving XML schema and a set of interaction classes which manage translation to and from CAD and analysis packages. Current analysis programs support include Radiance [103], EnergyPlus [101] and CAD programs such as Rhino [84]. Although many of these connections are not feature-complete they do allow the coupling of design generation and design analysis tools, potentially with optimisation software. However there is no overarching computational framework to "drive" this interoperability framework, instead it relies on custom scripts written by the practitioner. In comparison with Industry Foundation Classes we see that the DesignLinkSDK supports the interchange of data which is not currently supported by IFCs but is critical to analysis programs. While IFCs develop to support a wider range of information they are unlikely to become broad enough to deal with every form of design and analysis data involved. The DesignLinkSDK approach is aimed to be more agile and flexible to project needs than the more robust IFC standard. Further discussion regarding the approaches can be found in [2].

\subsubsection{Geographic Information Systems}

Geographic Information Systems (GIS) store large amounts of information on geography of a region, its features, buildings, infrastructure and so forth all of which are accurately geo-located. There are a number of such database systems such as ArcGIS [33] and PostGIS [74]. These store higher level information about a city scape, for example the shape of each plot of land or building outline, along with various meta data such as the type of building, its height and so forth. While such an approach cannot replace the detailed geometrical file formats of Collada and IFCs nor the detailed interconversion supported by the DesignLink SDK, they do provide a means of containing design details in a single standardised location with clear access requirements. GIS systems act not only a data repository but also enable a number of complex analyses such as identifying whether each house in a suburb is within walking distant of a bus stop. These analyses are undertaken in complex GIS workbench software such as ArcGIS [33]. Unfortunately GIS systems are not normally used by the design team within urban masterplanning they are used rather for documentation, detailed draft- 
ing and analyses during more detailed masterplanning and then by GIS professionals on the analysis team. This presents an interoperability challenge with the designer's CAD tools and GIS systems. There are a number of tools for addressing this challenge including FME.

\subsubsection{Feature Manipulation Engine (FME)}

FME (Feature Manipulation Engine) by Safe Software [87] is a geographic data workbench and toolkit. It contains a wide variety of over 300 data readers and writers along with tools for transforming, cleaning and validating data. The desktop version of the tool acts as a workbench allowing visual programming of data transformations through a drag and drop interface. The server version of the tool allows automated, large scale transformations of data and can provide federated access to data. The software primarily supports GIS data formats, however there is increasing support for CAD and BIM data formats. The software does not natively support automating analyses although this can be done through custom scripting. The framework's commercial success shows the reality of the interoperability challenges in this industry and the potential benefits from resolving them.

\subsubsection{Conclusions}

There is a substantial known problem with interoperability within the AEC industry. The range of solutions and standards discussed in this section show the difficulty of the problem and the breadth of the integration challenges across so many sectors of the AEC industry. To address this there are three basic approaches:

1. Standards based (Collada, CitygML, gbXML, IFCs) - each of which cover a different slice of the industry. However none of these is sufficiently broad to cover the scope of urban masterplanning. Similarly none are able to cover the range of detail that a masterplan design will go through from concept design through to detailed design and construction. The closest standard is CityGML with it's five levels of detail. All of these standards struggle to store the breath of analysis data which will be created during the masterplanning process. An example might include vector fields for a wind flow analysis, false colour maps for lighting and a detailed district energy system schematic. While continued development and increased adoption will continue to alleviate the challenges of interoperability there still remains substantial scope of research and improvement.

2. Database based (BIM and GIS) - These approaches aim to centralise data in a single curated repository accessed by practitioners. While GIS data tends to be larger scale it supports a wide range of disciplines. On the other hand 
BIM models support detailed design across a range of mechanical, structural and construction disciplines. These platforms are only as good as the standards that underpin them (for example IFCs in the case of many BIM servers). In the context of urban masterplanning there remains an additional challenge in that early stage design tools do not support such database platforms. This precludes their use in early stage analysis cycles except after costly conversion processes. For detailed design a database approach based on industry standards is likely to be very successful [13].

3. Transformer based (DesignLinkSDK and FME) - These approaches are a key to covering the variety of interoperability problems within the industry as well as providing quick conversion between the plethora of interoperability standards. These frameworks will however not resolve all incompatibilities between formats however a workbench like scripting environment is one key method for aiding these transformations. However the challenge of high user skill remains as a barrier to the wider adoption of these frameworks.

Hence we see that while interoperability challenges within and increasingly between disciplines are being bridged by industry standards there remains a substantial challenge. The use of databases for storing design and analysis data is one key technique as indeed are format transformations engines such as FME an DesignLink. The approach taken by FME is perhaps closest to providing improvements in the mismatch between the design and analysis cycles, however it does not include explicit model automation support. Thus there remains a key missing component to resolving the interoperability challenges in this industry. Namely the use of computational frameworks to bridge the gap between design and analysis programs allowing the driving of analysis models from design data. These frameworks must support industry standards, however since there is no one standard which covers all aspects of the industry, analysis frameworks should aim to be standards independent. The use of database models looks set to be a key technological driver in the future of this industry as we move away from single file designs to server stored design databases such as GIS or BIM systems. This will mean integration and query languages for these databases will become increasingly important [19] [70]. Finally advantage must be taken of the complex work undertaken to create transformation engines between different standards within the industry which will further enable practitioners to use the best tools for the task at hand.

\subsection{Conclusions}

Within the AEC industry there remain many challenges preventing a rapid design and assessment cycles producing well-performing buildings. Many of these challenges stem 
from the fragmented structure of the industry and the breadth of disciplines involved. The primary challenge remains the lack of a rapid analysis cycle which is in sync with the design cycle and capable of producing insight in a timely manner to the next design iteration. Underlying these challenges are a number of computational problems. These include model automation, interoperability problems, model integration and supporting practitioner led design space exploration.

Learning from other industries, we see the automotive and aeronautical industries already have advanced computational platforms for analysing designs and undertaking design studies. These are successful platforms which integrate well with the ecosystem of tools and analysis models. They are underpinned be computational techniques for model automation, integration and particularly optimisation algorithms and Design of Experiments (DoE) techniques for exploring the design space. However despite investigations into applying these tools to the AEC industry [36] these tools have not successfully transitioned to the AEC industry. Reasons for this include a lack of support for the AEC's ecosystem of tools to challenges of interoperability and the sheer range of disciplines involved. There is also reason to believe that the focus within these frameworks upon analyses as first class citizens in the data model to the exclusion of the design is a barrier to the uptake of these systems within the AEC industry.

Following this we have seen two competing drives within the AEC industry for the use of computation to resolve the lack of synchronisation between the design and analysis cycles. From the analysis side we have the rise of Computational Design Optimisation which uses genetic algorithms to partner parametric design tools with analysis models to produce well-performing designs. While these tools can successfully refine a design, their ability to generate new designs is not clear, and at best these techniques will remain only one of several a design tools rather than solution to the challenges the industry faces. From these techniques we see the value of design space exploration although the optimisation is in some sense not systematic and so fails to give insight into the design as a whole. Similarly we see the effectiveness of automated and integrated analysis models. Performance trees are another interesting technique used to good effect in this area. It may prove possible to re-purpose this technique for diagnosing design performance across disciplines or a design decomposition. Thus we see several techniques which should help to resolve the challenges identified in this industry.

The second development attempting to address this mismatch in design and analysis cycles is the use of (simple) analyses directly within the design tools themselves. This gives a great deal of benefit to the designers and shows the great potential of unifying the design and analysis cycles in the AEC industry. Particularly we see rapid feedback as enabling the designer to explore the design space to a greater degree than previously possible. However there remains limited computational underpinning to these tools. By this we mean that design space exploration techniques are not employed, nor is there 
the potential to integrate further tools into the framework. There is indeed a similar challenge to the CDO studies which are frequently scripted together ad-hoc. Hence we see a need for research into bringing together best techniques and data models from both approaches into a neutral framework able to benefit both designers and engineers.

A further area of related work comes in the form of projects to address the well known interoperability challenges within this industry. A plethora of industry standards have developed each covering a sector of the industry. As these standards have developed to incorporate more design and particularly analysis data they are better able to help bridge the gaps between the design and analysis cycles. However no single framework is able to cover the entire industry and limited adoption of frameworks precludes the solution of these challenges. Hence the use of database-based formats has aimed to centralise storage away from proprietary standards and allow access to multiple practitioners as required. However still we find that none of the standards are able cover the design cycles from early concept design to detailed design and construction. This necessitates costly redrafting and changes of design tools. A less considered aspect of the interoperability challenge is frameworks for conversion between formats (such as DesignLink and FME). While these can help within interoperability challenges and ease some of the time consuming tasks between the design and analysis cycles they do not provide the unification of the two cycles which is needed within this industry.

In considering these challenges and the attempts to address them we see there is a clear research gap. Namely a lack of investigation into the computational techniques for unifying the design and analysis cycles particularly on an urban masterplanning scale. To undertake this there is need for investigation into effectiveness of computational techniques for creating design insight which are applicable to the urban masterplanning community.

This computational framework should be open, support standards, yet be standard independent. It should enable automation of analyses and design space exploration either by automatic means or driven by the user. Techniques such as design of experiments and sensitivity analysis will be key to this (see section 2.4 (p51)). Integration of analysis models has the potential to provide great insight [36].

Given that there is limited research into the computational platform required to bridge the gap between the two cycles a platform should be developed to explore the efficacy of computational techniques and data models. Hence the best option is to sit neutrally in the intersection of the design cycle and analysis cycle rather than subjugating one to the other. This enables each cycle to continue undisturbed in its normal workflow, however with the computational support to provide greater design space exploration and more rapid analysis results and greater design. The platform should utilise the best techniques identified during this chapter to providing a computational foundation for computationally unifying urban masterplanning. 


\subsection{Hypotheses for computationally unifying urban masterplanning}

Having explored the computational challenges in urban masterplanning and numerous techniques for addressing the challenges from a wide variety of literature we now consider a number of hypotheses for techniques and approaches required for computationally unifying urban masterplanning effectively.

The goal for these techniques is to identify which will prove effective at resolving the computational challenges observed and producing insight for designers. We aim to answer our research question:

\section{Which computational frameworks and techniques are necessary and effective in creating design insight in the context of urban masterplan design and analysis?}

\subsubsection{Approaches}

Underlying the techniques used to address these challenges are a set of more general beliefs about the approach and solutions best employed. The author proposes the following principles to underpin computational approaches to unifying urban masterplanning:

- Insight not Optimisation - Given that all architectural analysis is undertaken to give insight for human decision making, this author believes that the key critical goal for computational research in the AEC community is to produce better insight for practitioners into their designs and the design space. In the literature much emphasis is placed on computational design optimisation [38] [89] [17] as the key driver. However this author believes this should be seen as one approach among many to produce insight for designers. Hence the key is to signpost the right way through the design space for engineers, architects and clients to go rather than attempting to find it using optimisation.

- Standard Independent - Given the plethora of standards and interoperability frameworks covering various slices of the industry, computational frameworks aiming to address the challenges in the AEC industry must be capable of supporting many standards and not be tied to any particular standard.

- Modular with Loose Coupling of analyses - The disparate nature of the software products used by the many disciplines involved in urban masterplanning mean that it is important that the analysis modules used can be interchanged to suit the needs of the project and the practitioners hence although tightly-coupled 
integrated models may be more accurate, loosely-coupled models are likely to be more adaptable to the needs of the industry.

- Use Existing Analyses - Given practitioners' experience, the cost of model development and, in some cases, model certification; it is important that computational frameworks integrate with existing models.

- Unified Data Model - A single data structure should be used for storing design and analysis data together into a flexible representation of the design would enable a simple model for adoption by practitioners.

- User Driven Design Space Exploration - Computational frameworks must allow designers and engineers to identify the effects of design decisions by allowing exploration of aspects of the design space they are interested in.

Specifically these approaches will motivate the testing of a number of specific computational techniques.

\subsubsection{Techniques}

There are number of computational techniques which should be explored with a view of providing greater synchronisation between the design and analysis cycles and producing more design insight. These techniques have proved effective in the literature in this chapter and should be adapted into the context of the AEC industry:

- Automation - To avoid the challenges of a slow architectural analysis cycle, automation of architectural analyses must be applied to give a quick enough turn around to fit with an architects cycle and deliver design space exploration techniques.

- Model Integration - Enabling the integration of models together allows their capabilities to be combined, enabling greater insight into the design while providing greater accuracy by automating what may previously have been a manual process.

- Scenario Analysis - Allowing engineers, designers, architects and clients to explore analysis scenarios; enabling comparison of design options and strategies under different conditions to make the best decisions.

- Design Decomposability - Decomposing a design into its constituents (e.g. suburbs, blocks and buildings). This would enable more detailed design analysis than currently possible over multiple scales. It should also support increasing design information over project life cycle. 
- Multi-Scale Analysis - Due to the nature of the analyses carried out, some will be undertaken at the development scale (e.g. windflow) while others will occur at the building scale. This necessitates the need for multi-scale analysis. Particularly interesting is enabling different analysis scales to work together. For example a per-building energy demand model providing demand forecasts to a district energy analysis model.

- Design Space Exploration - The ability to create, analyse and compare many different designs under many different scenarios is critical to the design of wellperforming developments. Design space exploration should:

- Be User Driven - Enabling the expertise of practitioners to drive the exploration of the most interesting design questions and directions.

- Be Model Driven - Enable exploration of the insight embedded in analysis models to explore potential design improvements by for example exploring carbon mitigation strategies.

- Use Sensitivity Analysis - Given the complexity of design, identifying which of thousands of parameters are the most important for improving design performance is a crucial goal.

- Use Optimisation - Optimisation can prove extremely effective at improving a design under specific circumstances. Often, once the main design is set, optimisation can be used to whet its performance.

- 3d Visualisation - Since architectural designs are inherently geometric performance must visualised in the context of design geometry; showing how performance is attributable to design features. Specifically visualisation must be:

- Interactive - Allowing users to engage with the design and its performance

- Integrated - Allowing multiple discipline's performance figures to be displayed and explored a good example is the $3 \mathrm{~d}$ Urbanism project described in section 2.7.1 (p66).

- Quantitative - Performance of the masterplan must be visualised quantitatively enabling direct exploration of trends and the reason for them.

- Comparative - Visualisation must enable the user to choose between design options and scenarios, to see the effect of design changes and guide the design process.

- Performance Trees - Are widely used as an effective means of creating a composite performance scores across KPIs from multiple disciplines [17] allowing drilling down through performance to identify root causes of problems and scope for improvement. 
Having explored a number of computational data models and techniques for addressing the challenges in urban masterplanning we now explore a framework which introduces a new data model and allows the investigation of the efficacy of a number of techniques to address these challenges which will be explored in a live case study in Chapter 4 (p139). 



\section{HierSynth}

This chapter introduces the HierSynth framework and its data model which attempts to meet the challenges identified in Chapter 2 (p37) for a framework for computationally unifying urban masterplanning. We introduce a novel data model and execution framework that helps meet these goals and discuss its use for model integration, scenario composition and sensitivity analysis. Chapter 4 (p139) describes in detail a case study application of the HierSynth framework to a live masterplanning project in collaboration with Arup.

\subsection{Overview}

The HierSynth computational framework integrates city-wide analyses into a common platform allowing automated analysis of designs under multiple scenarios. HierSynth hierarchically decomposes a design for increasingly detailed insight as designs develop. The framework includes many tools to enable synthesis of insight into the design for the design and analysis teams.

The framework is created with the intention of bridging the gap between the design and analysis communities. It is to be used as a testbed for a variety of computational techniques to determine their efficacy in producing design insight for both communities.

\subsection{HierSynth}

At a high level Fig. 3.2 (p87) shows the HierSynth framework and its position in the urban masterplan design/analysis cycle described in Fig. 3.1 (p86). HierSynth is aimed to sit between the analysis and design cycles enabling collaboration and coordination between the two. In this sense HierSynth is a communication tool between these two communities.

Specifically HierSynth reads design files such as 3d geometry and land use schedules along with information about analysis scenarios (e.g. weather data or energy use intensities). HierSynth then automatically runs a number of user specified, industry standard, analyses and collates the analysis results. These results are then automatically synthesised into insight into the design by various tools and capabilities within 


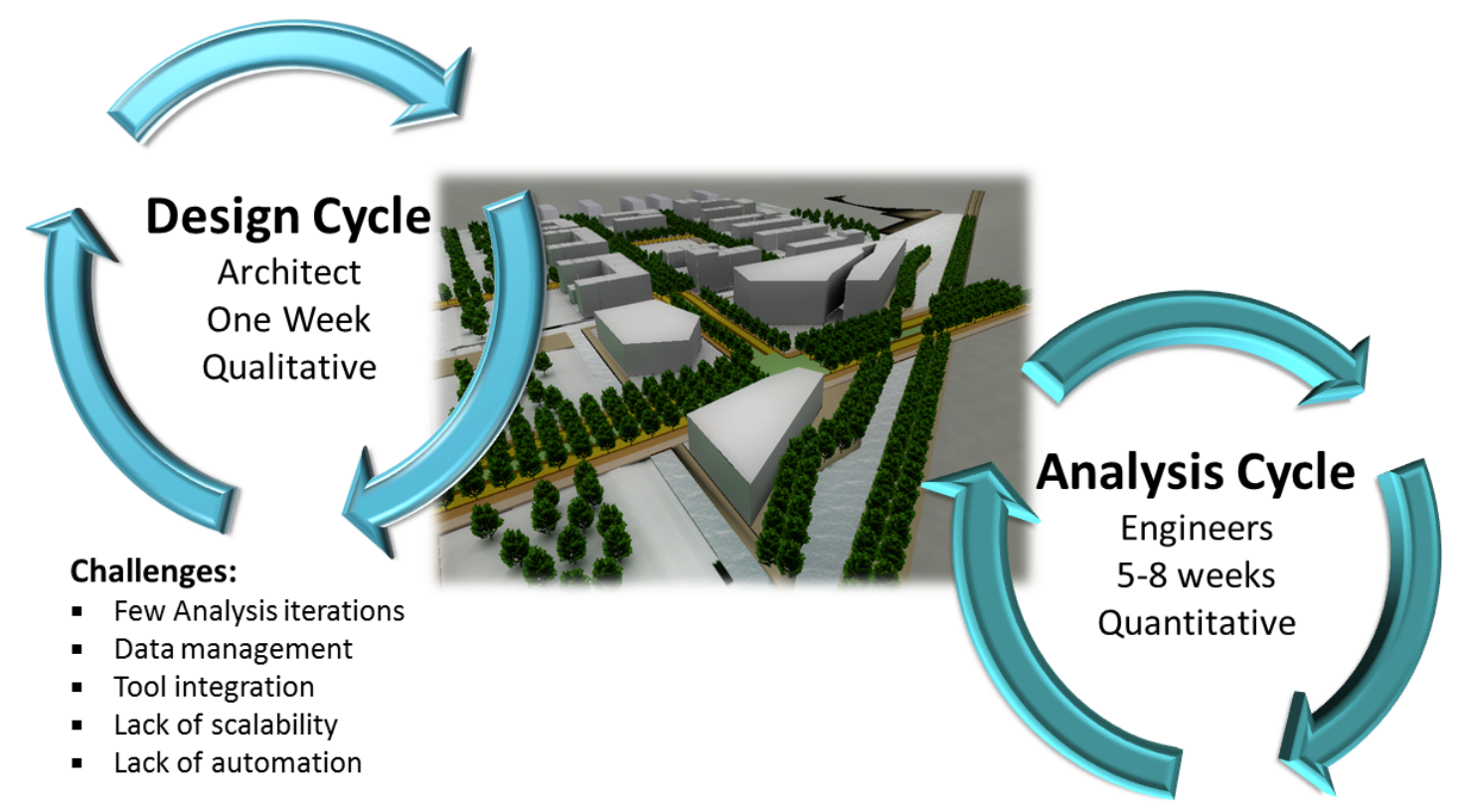

Figure 3.1: The central challenge that HierSynth aims to address is the lack of synchronisation between the design and analysis cycles in urban masterplanning. As quantified by [35] the analysis cycle can take 5-8 times longer than a design iteration. HierSynth enables exploration of the computational techniques required to resolve these challenges and generate design insight.

HierSynth such as performance trees for every design element, 3d visualisation, graphing and sensitivity analysis.

This chapter will discuss the HierSynth platform in detail from its design goals to the data model, its performance optimisations and implementation highlights.

\subsection{HierSynth Design Goals}

The goals of the HierSynth framework were as follows:

- Hierarchical - Enable detailed insight via design decomposition enabling the production of detailed analysis results on multiple scales. Design decomposition might be by geographical containment (buildings, blocks, streets, districts, city) or by thematic category (building type) or a mix of the two. Some analyses will occur at district level others at building level. As the design develops, more detailed layers of decomposition will be available.

- Synthesis - provide tools for the synthesis of insight from analysis data, from graphing to visualisation to sensitivity analysis.

- Integration - To enable the integration of different analyses together using HierSynth. This will provide greater analysis capability and insight into the design. 


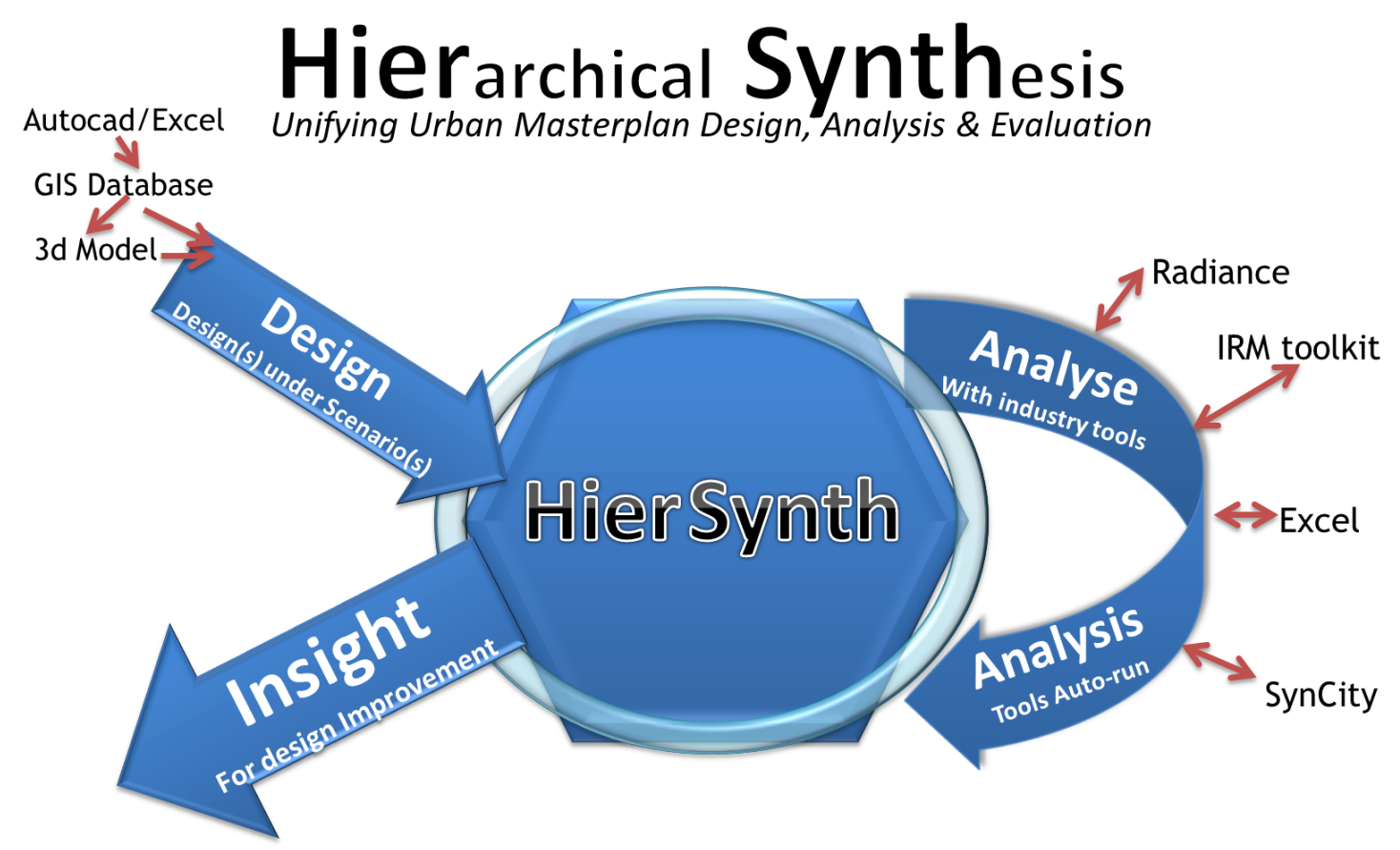

Figure 3.2: Overview of HierSynth platform

- Extensibility - To enable further development of HierSynth via plugins, data analytics and visualisation providers. To enable scripting within the data model wherever possible. This is required due to the plethora of design and analysis programs and their file formats.

- Performance - Since a goal of the framework is to reduce the time taken for an analysis cycle, performance is critical. Key to this is parallelisation of analyses.

- Independent - The framework should be independent of any one analysis or design tool or one particular discipline. This will enable it to be adapted to integrate any design or analysis tool using any schema or ontology. This is important due to the fragmentation of disciplines and tools in this domain.

We will now discuss the data model of HierSynth which was developed to provide a test bed for techniques to help computationally unify urban masterplanning. 


\subsection{HierSynth Data Model Executive Summary}

The core of the HierSynth framework is its hierarchical data model. This allows the composition of design queries, design components and analyses to form a tree data structure which allows design decomposition and analysis. The framework is then able to compose these trees to generate scenarios for evaluation and to conduct design space exploration and sensitivity analyses.

The key novelty of this data model is in its reconceptualization of a workflow graph (such as the one shown in Fig. 3.3 (p89)) by composing it with tree based design decompositions more commonly found in industry interoperability file formats (of a similar form to that of Fig. 3.4 (p90)). This is achieved by composing a hierarchy of design queries, templates and analysis nodes which, when combined through a novel dataflow algorithm form, when executed, creates a design hierarchy annotated with analyses to be performed. During execution queries are evaluated and their results (returned records) are represented in the tree as a collection of child nodes which are instantiated by child templates (for example a suburb node querying for the buildings it contains). These templates may include analysis nodes which are also instantiated for each returned result. This enables simple multi-scale analysis at all levels of detail, a key feature for use in the realm of urban masterplanning. At each node in the hierarchy and for every scenario evaluated a performance tree is created. This enables the consideration of many performance concerns in a single performance figure through repeated aggregation in a tree like structure. Many methods of aggregation are proposed in the literature. However for the first time we present a framework with performance trees for every design component and show mechanisms for performance trees to aggregate performance from the performance trees lower in the design hierarchy. This should enable rapid identification performance problems and opportunities across disciplines and within the whole design decomposition.

The data flow algorithm enables scenarios (key-value dictionaries containing design data and analysis configuration data) to flow from the root to leaves of the tree structure. At every branch the scenario is forked to allow different branches to have different scenarios specialised to the part of the design they represent. This is achieved by enabling each node to "patch" (update) the scenario, with a patch being visible to all descendant nodes. This patching and branching of the scenarios to create more specialised scenarios is utilised under a reconceptualisation of the hierarchical data model to provide scenario generation capabilities. Such uses of the data model are used sequentially with standard implementations to allow the specification of design space exploration experiments to answer the "what if" questions frequently asked by engineering teams. This framework combined with techniques including sensitivity analysis and integrated $3 \mathrm{~d}$ visualisation, are applied to the discipline of urban masterplanning 


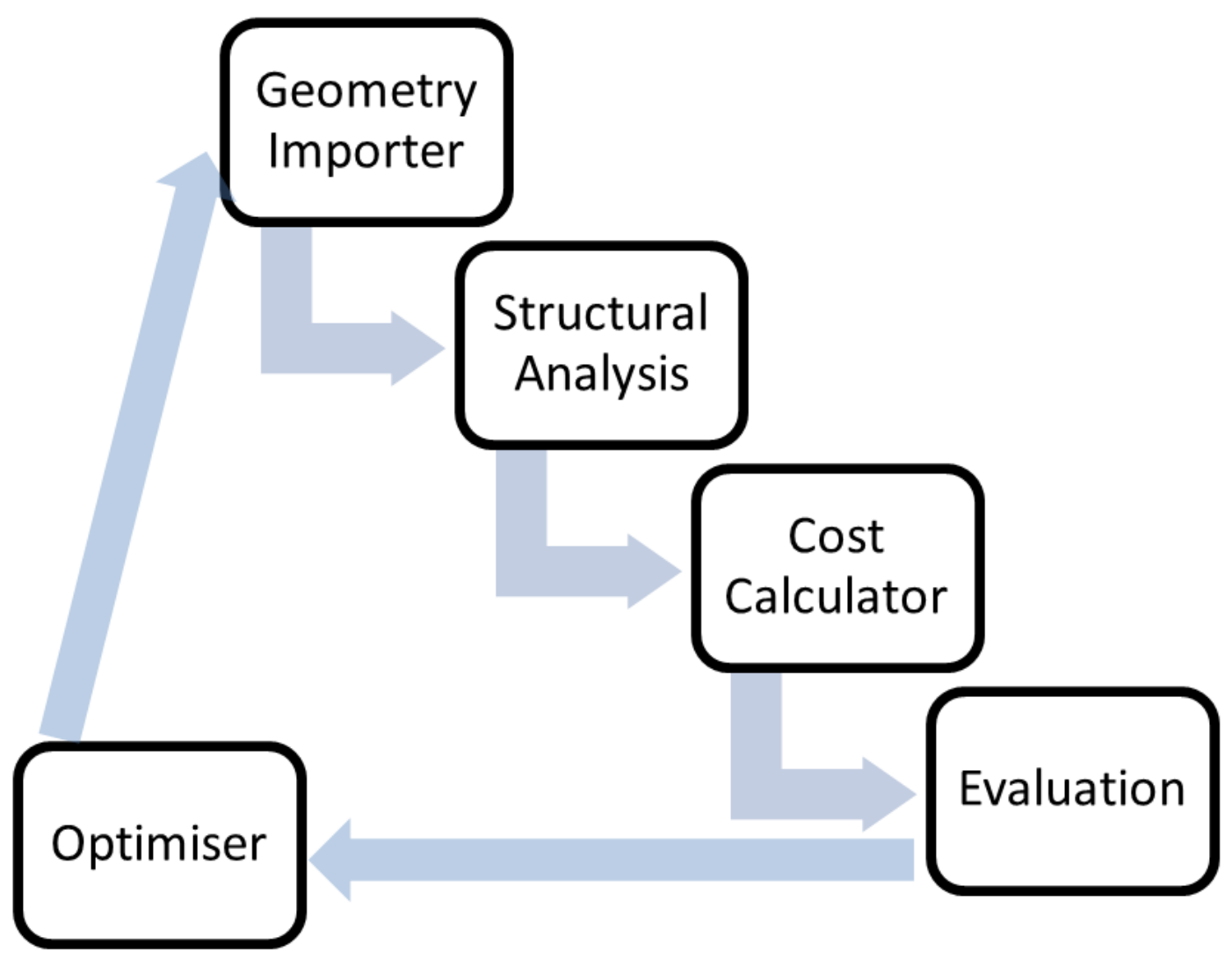

Figure 3.3: A conceptual model of a standard workflow model for optimising a steel structure. The composition of such a model with the design decompositions (see Fig. 3.4 (p90)) often seen in interoperability formats form the basis of the HierSynth data model which is shown in Fig. 3.5 (p91).

to bridge the growing computational gulf between architectural design and analysis teams.

\subsubsection{HierSynth Tree}

HierSynth's data model is based around a dictionary concept which contains key value pairs containing any form of data which can be serialised to XML. These dictionaries are used to represent "Scenarios" which contain design and analysis configuration data. The HierSynth data model centers upon the HierSynth tree, and example of which is shown in Fig. 3.5 (p91). A HierSynth tree consists of design queries, template and analysis nodes. The input to a HierSynth tree is a set of scenarios each consisting of a dictionary of key - value objects. These scenarios will contain all necessary configuration values for all design queries (e.g. CAD file locations) and all configuration needed for the analyses defined in the tree (e.g. weather data or energy intensities). The execution of a HierSynth tree consists of three phases. An expansion phase when all 


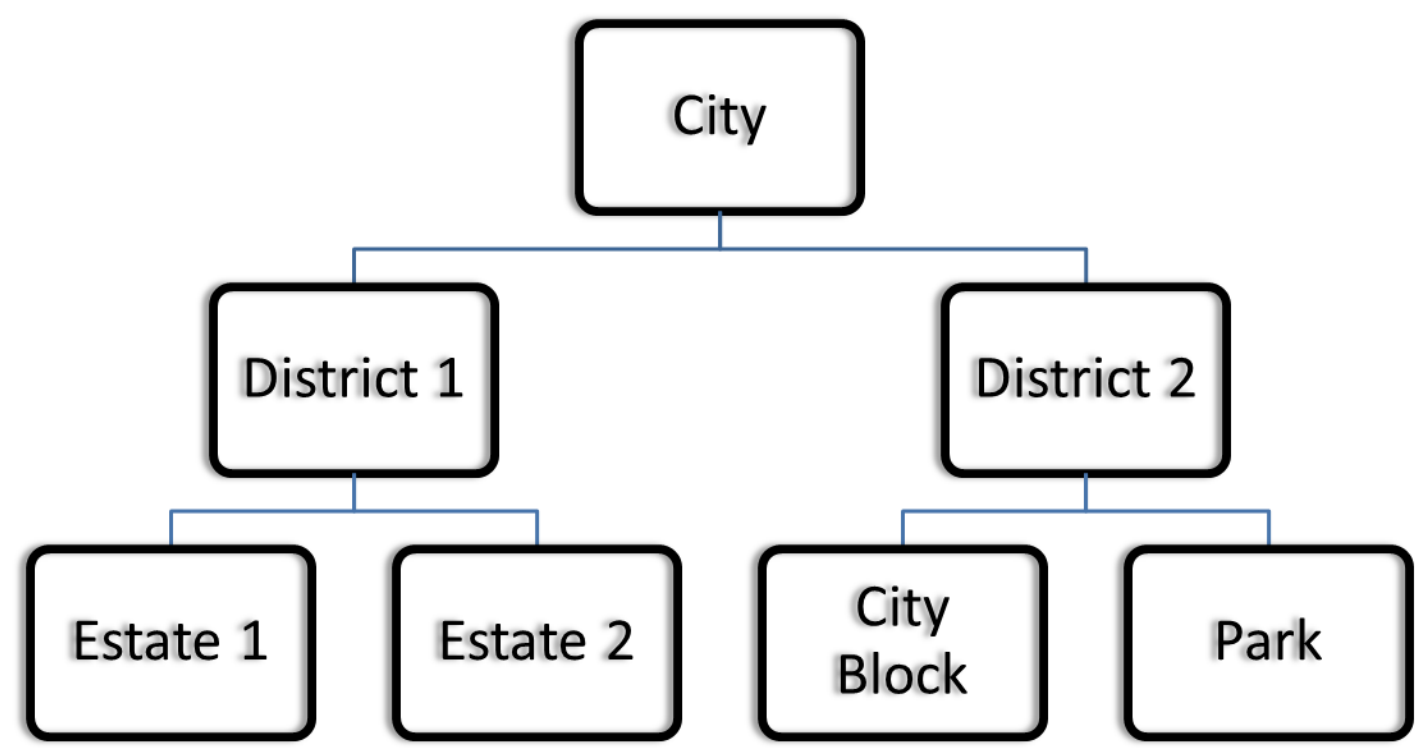

Figure 3.4: An example design decomposition of the type often found in interoperability formats within this industry. The composition of such decompositions with workflow data models (see Fig. 3.3 (p89)) form the basis of the HierSynth data model which is shown in Fig. 3.5 (p91).

queries are evaluated and the tree expanded to reflect the design and its decomposition specified within the tree (e.g. a city, all suburbs and all buildings within them). The execution phase runs all analyses and return results for storage in the tree. Finally an aggregation phase occurs to compute aggregate metrics (such as the average or total carbon emissions from a set of child "district" nodes) and performance trees for each district.

The expansion phase executes the queries within the tree and as shown in Fig. 3.5 (p91) instances the subtree of each child node of the analysis for each record which is returned. When executing under many scenarios a unique key for each record is used to match records across scenarios so that only one node represents a design component (each node may appear under many scenarios).

During execution the scenario is passed from the root node down through the tree and is optionally patched by each node as it is executed. The patch made at one node will be visible to that node and all its descendants. This allows specialisation of the analyses being carried out according to the tree decomposition. A simple example is shown in Fig. 3.5 (p91) where the information on each district is patched by the template nodes "District A" and "District B" which are generated by the "Query for Districts" node. These patches mean that the IRM [75] analyses for each district access information localised to that particular district. This technique allows hierarchies of queries and analyses to become successively more detailed (e.g. querying for buildings in a given suburb). Alternatively it enables different types of analyses to be undertaken 


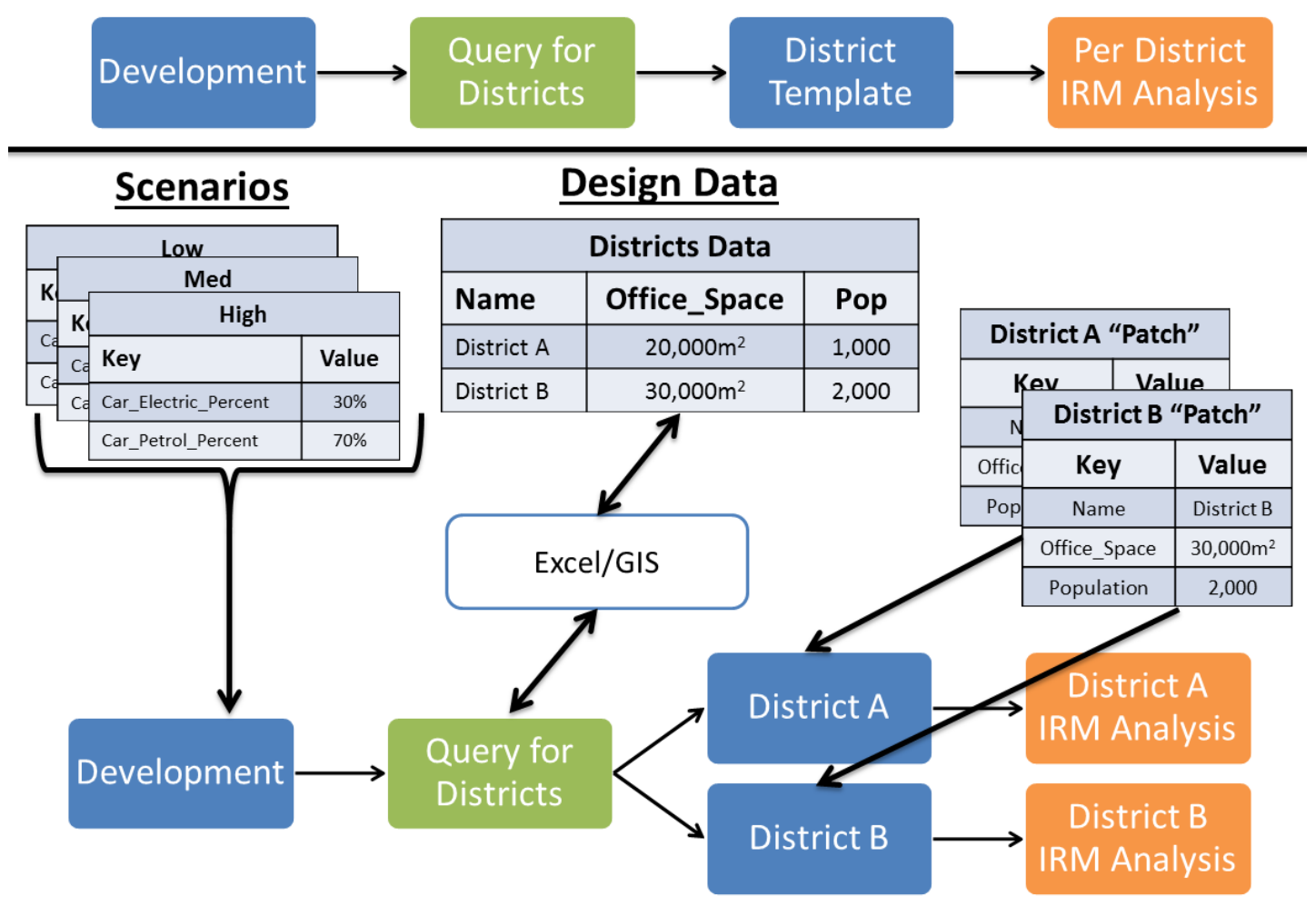

Figure 3.5: A conceptual model of a HierSynth tree before (top) and after expansion (bottom). For each initial scenario the tree is executed and the design query is evaluated querying the design for the districts it contains. The sub-tree of each query node is then instanced for each design component (district) returned. In this case an analysis query is then run to compute the carbon emissions from this district.

in different branches of the tree.

Execution normally occurs from leaf to root to enable larger scale analyses to use results from analyses of design components, however this can be reversed if required. A final synthesis stage then occurs to collate aggregate metrics back up the tree. At this stage performance trees computing a score or preference metric from many different analysis results is computed for each design component.

\subsubsection{Execution Chains}

Frequently HierSynth trees are used together to form execution chains. These enable one or more trees to create a set of design scenarios for analysis by a final tree. This promotes the reuse of analysis structures. Within an execution chain is it possible to splice a sensitivity analysis for greater design insight as discussed in section 3.14 (p127).

Scenario generation occurs by using the branching flow of data within a HierSynth tree from root to leaf coupled with recursive patching of the scenario data to create new scenarios at the leaves of a HierSynth tree. As shown in Fig. 4.3 (p150) design 
and scenario queries can be used to create and most importantly compose orthogonal concerns to cover a larger part of the design space; in this case designs and energy strategies. By changing the execution order of nodes (to run root to leaf) it is possible to use results from analyses within the tree to create new scenarios (as shown in Fig. 3.15 (p119)). Similarly generator nodes within the tree can create sets of children with specific scenario patches to explore a range of options. Scenario composition enabled through execution chains was found to be a key technique for producing design insight.

\subsubsection{Core Concepts}

The HierSynth framework and data model has the following core concepts. Each of these, and their inter-relation will be explored during this chapter. Each has a key task in enabling the adoption of computing techniques within the AEC industry and the move to computationally unify urban masterplanning.

- Scenarios - these are dictionaries of key-value pairs and contain a mix of design data and analysis configuration data. Example scenarios might include configuration data for analysing under "Flooding" or "Drought" conditions. Scenarios are the inputs to a HierSynth tree. Scenarios may be generated using scenario generation trees in an execution chain.

- HierSynth Tree - This is the central data structure within HierSynth. It consists of a mix of Analysis, Query and Template nodes. HierSynth trees are executed under many scenarios. Many HierSynth trees can be chained together to form an execution chain.

- Query Node - These nodes query data-sources external to HierSynth; reading design data and analysis configuration data. e.g. the number of design files in a directory; a GIS database, a CAD file or an Excel land use schedule. Query nodes generate many child nodes by instantiating many child template nodes. Nested queries model Design Decomposition.

- Template Node - These are basic HierSynth tree nodes and the basis of Query and Analysis nodes. They store design information and analysis results. They represent design elements (e.g. districts or buildings) and may patch the scenario during dataflow with more specific design or analysis data.

- Design Decomposition - The technique of using nested queries to build a treelike representation of design elements. For example decomposing a masterplan as "City $\rightarrow$ Districts $\rightarrow$ Buildings".

- Analysis Node - These specify the running of external analysis programs to analyse sets of design elements represented by template nodes. 
- Scenario Data flow - Scenarios flow from root to the leaves of a HierSynth tree. Scenarios may be patched during this flow.

- Scenario Patching - During data flow from root to leaves a scenario may be "patched" with additional or updated data by any node; such changes are visible to descendant nodes.

- Tree Expansion - This is the process of evaluating all of the query nodes in a HierSynth tree. During expansion the HierSynth tree is expanded to reflect the design decomposition specified by the query and template nodes in the tree. Analysis nodes are similarly instanced for each design element.

- Tree Execution - This is the process of executing all analysis nodes in the tree.

- Tree Aggregation - This is the process of aggregating the performance of each element in the design decomposition into aggregate performance figures. For example calculating the total or average carbon emissions from a set of districts. Aggregation happens for many Data Layers.

- Data Layer - A data layer is the set of values for a particular performance metric stored within a HierSynth tree. One value (per scenario) is stored per node in a HierSynth tree. Example data layers include "Percapita carbon" or "Energy Consumption". Data layers are calculated during Tree Aggregation and store overall performance for each level of a Design Decomposition. Data layers are used to form Performance Trees.

- Performance Tree - A technique for aggregating many aspects of a designs performance into a single figure. Many techniques for aggregation are possible, either aggregating quantitative performance or synthetic measures of preference. In HierSynth performance trees are computed for each node and are formed from many inter-related data layers.

- Execution Chain - A connected set of HierSynth trees, several of which will generate scenarios for analysis by a final analysis tree.

- Scenario Generation Tree - A HierSynth tree built so that the Scenario Patching occurring within the tree is used to generate novel scenarios at the leaf nodes which can then be extracted and analysed by the next HierSynth tree in the execution chain.

Fig. 3.17 (p126) shows the GUI of the HierSynth platform. Currently HierSynth is a stand alone desktop application which contains a central core and many plug-ins to support a variety of data sources and analysis applications. HierSynth is deliberately 
standard independent and is not tied to one interoperability format since no single format will cover the breadth of urban masterplanning design and analysis. A mathematical expression library enables simple scripting and extensibility throughout the data model.

\subsection{Advantages and Disadvantages}

In this section we list some of the challenges and advantages of the HierSynth data model as it will be presented over the coming chapter.

\subsubsection{Advantages}

- Ability to deal with many types of data. As discussed in section 3.6.1 (p96) HierSynth scenarios may contain many types of data from strings to images.

- HierSynth includes a simple scripting language, this is discussed in section 3.6.2 (p97).

- HierSynth's expansion algorithm enables querying of design files and the creation of a design decomposition reflecting a "view" or "slice" of the design which the practitioner wishes to see. This reduces the complexity of the design information presented and is achieved through the use of query nodes as discussed in section 3.7 .1 (p102).

- The scripting language in HierSynth enables extraction of required inputs from a HierSynth tree. This enables a degree of consistency checking and is described in section 3.6.6 (p100).

- The aggregation phase during execution of a HierSynth tree enables collating of metrics across the design decomposition for higher-level insight into design performance. This is discussed in section 3.7 .3 (p108).

- HierSynth aggregation enables the construction of performance trees for every node in the design decomposition. This enables consideration of performance across disciplines at every level of the design. The mechanism for constructing such performance trees is discussed in section 3.8 (p108).

- A key concern of workflow systems is performance. Through its hierarchical data model HierSynth provides ample scope for parallelisation. This is discussed in section 3.12.1 (p122).

- Similarly HierSynth provides a caching framework as discussed in section 3.12.3 (p125). This works closely with an application sharing framework to mediate 
contention for resources and licenses of the complex analysis programs used in this industry. More details can be found in section 3.12 .2 (p124).

\subsubsection{Disadvantages}

- Restricting users to a tree structure precludes some of the flexibility of traditional graph-based workflow systems. To address this HierSynth provides "Execution Links" to enable inter-node communication. This is discussed in section 3.9 (p111).

- Linking the workflow structure directly to the design decomposition structure means that different designs will cause workflow and make comparison difficult. This is addressed through the use of a novel expansion algorithm discussed in section 3.7.1 (p102) which aims to ensure that identical design elements are represented by a single node across multiple scenarios.

- Unlike the many product optimisation frameworks discussed in section 2.4 (p51) the HierSynth data model does not easily allow for optimisation or design space exploration studies. To address this HierSynth trees are used to generate new scenarios (as discussed in section 3.11 (p120)) and are used in series with sensitivity analyses spliced before and after the analysis tree. This is discussed in detail in section 3.10 (p114) and in section 3.14 (p127).

Further discussion of the HierSynth framework can be found by considering its context in section 3.17 (p134) and subsequently considering its performance upon an industrial case study in Chapter 4 (p139). The performance of HierSynth in this case study is discussed in section 4.15 (p199). However we now turn to provide a more detailed description of the HierSynth framework. 


\subsection{Full Data Model}

This section explores the data model behind HierSynth. At the heart of HierSynth is a tree-based data model which enables the hierarchical decomposition of the design and the synthesis of insight from analysis modules run on these design elements. First we will explore the data model for analysis scenarios, then how a HierSynth tree is put together and how scenario data flows through this tree. Then the algorithm for executing a HierSynthTree will be presented including the use of performance trees to generate insight. This will give a basic understanding of the data model in HierSynth.

\subsubsection{Data \& Scenarios}

The HierSynth data model is based upon a flexible representation of data due to the plethora of designs, disciplines, geometry and analysis models within the urban masterplanning community. Whilst many projects standards, interoperability formats and ontologies existing within this industry (see section 2.8 (p71)) there is no single format which will sufficiently cover the breadth of disciplines involved. The cost of creating or modifying a format and ontology to be broad enough to cover the discipline is prohibitive. Research continues into addressing these problems, developing standardinterconversion techniques; however these are not widely used in industry. Similarly in the field HierSynth was applied to, no industry standard formats were in wide use by practitioners (with the exception of Collada [55] 3d format). Hence, whilst the HierSynth data model has the capacity to support reading each of these formats, internally a more basic data format is used. Thus HierSynth supports basic data types via an expression language. This allows any form of data to be stored that can be serialized to $\mathrm{xml}$ (e.g. images as binary strings). This should provide enough flexibility to represent the different data types used in various industry standards which HierSynth will interface with.

These data types are used to form a "Variable Map" which is simply a dictionary of named data. This data forms the basis of a design scenario for analysis. Variable names within this dictionary are encouraged to be named in a structured format in this format:

\section{"FloorSpace_Housing_GFAinM2", "FloorSpace_Office_GFAinM2"}

This enables the display of the variable map in a hierarchical form as shown in Fig. 3.6 (p97) which enables simple management of the often hundreds of variables involved in a design and analysis scenario.

Within the HierSynth framework variable maps are used to represent scenarios. Scenarios within HierSynth contain a mix of the following types of data:

- Configuration data needed to query design files. 


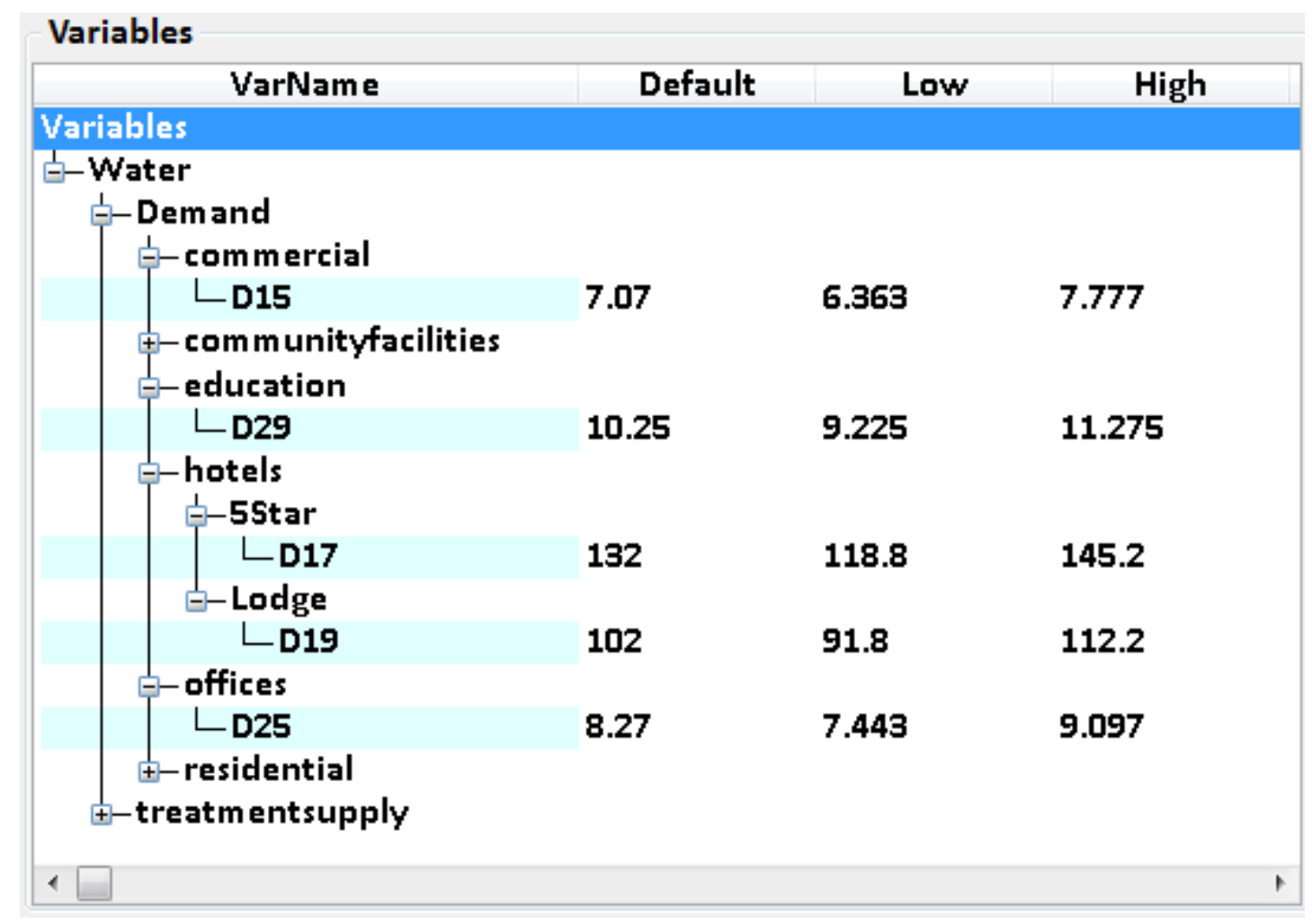

Figure 3.6: An example variable map displayed as a tree according to the names of its variables. Variable names are of the form "Water_Demand_Commercial_D15_units" or "Water_Demand_Education_D29". This provides a simple ontology and an easy way to navigate thousands of design and analysis assumptions.

- Design data and geometry.

- Configuration data needed to run analyses.

- Analysis assumptions (e.g. weather conditions).

Scenarios are the inputs to the HierSynth data model and they are modified within the data model. Scenarios may contain groups of expressions which are evaluated during execution using a worklist algorithm to resolve references between variables.

\subsubsection{Expression Language}

Throughout HierSynth an expression language is used for (simple) user scripting and computation. This enables extensibility and provides many capacities for users to customise HierSynth and its analysis modules. HierSynth uses a modified version of the NCALC [85] mathematical expression library. This was chosen in part because of its similarity to Microsoft Excel functions which practitioners within this industry are familiar with. Additionally its customisability to support different data types and the 
ease of adding new functions to the library were advantages. An example expression is shown below and is used to set up a number of design scenarios. Almost all configuration inputs in HierSynth are actually expressions. The expressions are executed in the context of the variable map at that node.

Design_Population $* i * i f\left(\right.$ EnergyStrategy_Name $={ }^{\prime}$ Good , 0.8, if (EnergyStrategy_Name $={ }^{\prime}$ Better' $\left., 0.7,0.6\right)$ )

This expression language was chosen in part due to its similarity with spreadsheet formulas. These are well known and widely used by almost all of the practitioners in engineering analysis and indeed by many of the design community. This familiarity should avoid any learning curve posed by other potential scripting languages. Similarly it should enable practitioners to quickly customise the HierSynth platform to meet their needs.

\subsubsection{Tree structure}

The central data structure in HierSynth is the HierSynth tree. It specifies how to query the design and how to analyse design components. It forms the basis of all design and analysis data storage. A tree structure was chosen since it enables design decomposition hierarchically. This enables more detailed design insight at multiple scales. This decomposition can be performed in a number of ways, perhaps per analysis discipline, per design element type or perhaps most commonly by geometrical containment (city, district, street and building). Such break down provides a simple way of drilling down into design performance to identify problem design components and areas for improvement. It also enables the amount of design data to grow as the design advances and becomes more detailed through the masterplanning process.

Fig. 3.7 (p99) shows an example HierSynth tree which seeks to analyse a city design by decomposing it by suburbs and buildings. Two analyses are to be performed, first a city wide lighting analysis using the open source lighting simulator Radiance [103] and secondly a per building sustainability assessment using Arup's Integrated Resource Management (IRM) model [12]. Each HierSynth tree is composed of a number of node types as shown in the right hand column of Fig. 3.7 (p99).

\subsubsection{Node Types}

The nodes of a HierSynth tree fall into three categories. Each category will have a number of implementations targeting different data sources or analysis modules.

1. Query Nodes - These nodes query design and scenario information from external data sources. They produce child nodes which reflect design elements. For example in Fig. 3.7 (p99) the suburb query will instantiate the suburb template for each suburb read from the design. 
Name

CityBySuburbandType

-Suburb Query

备-Suburb Template

占-Building Query Template

LBuilding IRM Template

Suburbs Radiance Node
Type

SimNode

ExcelQueryNode

ExcelQueryNode

GISQueryNode

ExcelNode

RadianceNode

Figure 3.7: An example HierSynth tree consisting of a number of design queries, several design element templates and an analysis node which also acts as a design element template.

2. Template Nodes - These nodes hold design data and frequently represent design elements (such as buildings) and are frequently instanced by query nodes.

3. Analysis Nodes - These nodes will run various analyses on various design elements. Analysis results are stored either in the design element nodes or in the analysis node.

Query nodes and analysis nodes are extensions to template nodes and can be used in place of template nodes at any point. As an example consider that the building template node in Fig. 3.7 (p99) is actually an IRM analysis node.

\subsubsection{Data flow}

A HierSynth tree will be executed for a number of analysis scenarios. These are variable maps as discussed in section 3.6.1 (p96) which contain all of the configuration data needed to query the design and to analyse it. For example the filename of the design to be analysed might be included in the scenario as well as the average monthly temperature of masterplan area which would be used to configure an analysis.

This scenario data flows down the tree from the root node to the leaves of the tree providing configuration data to all nodes. Each node of the tree has the ability to modify the scenario by adding variables or changing their values. These modifications will affect only that node and its descendants. This recursive patching enables each design element to modify the analysis context with specific information. For example in Fig. 3.7 (p99) the suburb template node will affect its building query child node 
so that it only queries for buildings in that particular suburb. This process enables increasingly detailed data to be stored in the scenario as it descends the tree.

A tree node may also modify the scenario for that node only. Finally nodes will also store analysis results as node "Outputs" which are separate from the scenario. These analysis results will be generated by the execution of the tree. HierSynth includes an interface for exploring this data flow through the tree model under all scenarios; this proves useful for debugging purposes.

\subsubsection{Analysis Requirements}

One major benefit of using the expression language discussed in section 3.6.2 (p97) is that it enables simple extraction of the inputs required to evaluate a formula. Hence it is possible to gain a list of required variables from a HierSynth node since all inputs will be entered using these expressions or read from the scenario variable map. We note that given the data flow of scenario data some of these requirements from a given node will have been fulfilled by its parent nodes (e.g. a template node and a parent query node). This enables a useful ability to check before execution that the initial scenario used for execution contains all of the required inputs for the analysis to proceed. Additionally this concept could be used to automatically integrate different analyses based upon a list of the variables they produce and consume, however this would require unit types to be recorded accurately and for the ontologies used by each model to be matched correctly.

\subsection{Tree Execution}

Following the overview of HierSynth shown in Fig. 3.2 (p87) the first task of HierSynth and its data model is to query the design and form an internal representation of it. The second stage of execution is to automatically run various analysis engines upon design elements at various scales and collate and store the analysis results. Finally the combined design data and analysis results are used to deliver insight to the analysis and design teams using a variety of insight tools. We will now explore the tree execution algorithm which follows the steps shown in Fig. 3.8 (p101).

The first of these steps is the composition of the analysis tree by the user to reflect a recipe for querying the design and producing a "view" of the design decomposed into it's design components. Annotated within this recipe are specifications of various analyses to undertake on various design components. All of these nodes are configured and composed into a tree by the user with configured nodes being re-usable in multiple HierSynth trees. This enables a library of configured general analyses and design queries to be maintained per project. It is likely there will emerge a number of "design patterns" for such HierSynth trees as common design decompositions and analysis integrations 


\section{Compose}

- Practitioner creates a HierSynth tree from configured queries and analyses, possibly using a library of standard configured queries and analysis.

\section{Expand}

- HierSynth executes design and scenario queries creating an internal view of the design in the HierSynth tree containing design data for each design element in the decomposition.

\section{Execute}

- HierSynth executes the analysis modules specified in the HierSynth tree to analyse the design elements at various scales. Analysis results are collated and stored in the HierSynth tree.

\section{Aggregate}

- HierSynth computes aggregate performance metrics at all levels of the design decomposition shown in the HierSynth tree. Performance trees are then created for each design component.

\section{Insight}

- HierSynth provides a number of techniques for drawing insight from the design and analysis data; including information visualisation, $3 d$ quantitative visualisation, sensitivity analysis.

Figure 3.8: An overview of the steps for evaluating a HierSynth Tree. From a user composing such a tree, through three steps of the execution algorithm to a final step allowing the user to utilise techniques in the framework for drawing design insight from the analysis results. 
are found. HierSynth supports this by storing and enabling the sharing of configured analyses.

The final step is the most interesting and involves the drawing of insightful conclusions from the design and analysis data stored within the design decomposition of the HierSynth tree. This may be as simple as identifying the worst-performing building in a district; or more subtle such as understanding the link between building orientation and water consumption. The framework provides numerous tools for aiding this process of extracting insight and aims to provide a testbed for exploring the efficacy of such techniques. Before discussing these techniques we will first explore the three steps of executing a HierSynth tree as shown in Fig. 3.8 (p101) .

\subsubsection{Expand Phase}

The first task of the HierSynth tree is to read in the design and its associated data. This is done during the tree expansion phase of execution. During this phase we can consider the HierSynth tree to be a formal description of the design hierarchy and how to query for its data. This is carried out upon all nodes starting with the root node and descending to the leaf nodes. During this phase all of the query nodes will be evaluated and the design will be read into HierSynth where an internal representation of the design is formed in the tree structure.

An individual query node will contain information on the source of data to query, how to perform the query and which source of data to return. This data source could be a GIS database, a CAD file, a spreadsheet table or some other source of data. The query will produce a set of records each representing a design element; perhaps a building and information on that building. Note that not all query nodes need actually query external resources some will generate a series of records using internal logic, perhaps by sampling from a statistical distribution or via a loop producing a set of connected records representing for example a range of values for the number of electric cars in the development.

As shown in pseudocode in Listing 3.1 (p104) the final step of the expansion algorithm is to instance the child templates. This happens for each child template node. A template will first filter the records it will accept then create an instance of the template for each of these records. These instances are added to the HierSynth tree as child nodes of the query. The original template node is removed. These template instances are then populated with their record's data as part of their variable mapping. When a template is instantiated the whole subtree that it roots is also instanced. This enables a building template to be instanced for each building in a suburb along with a set of child analyses to be carried out on a per building basis. Note that a query with multiple template child nodes may mean that a single record can instantiate multiple child nodes. We note that not all child nodes of a query will be templates requiring instancing; for 


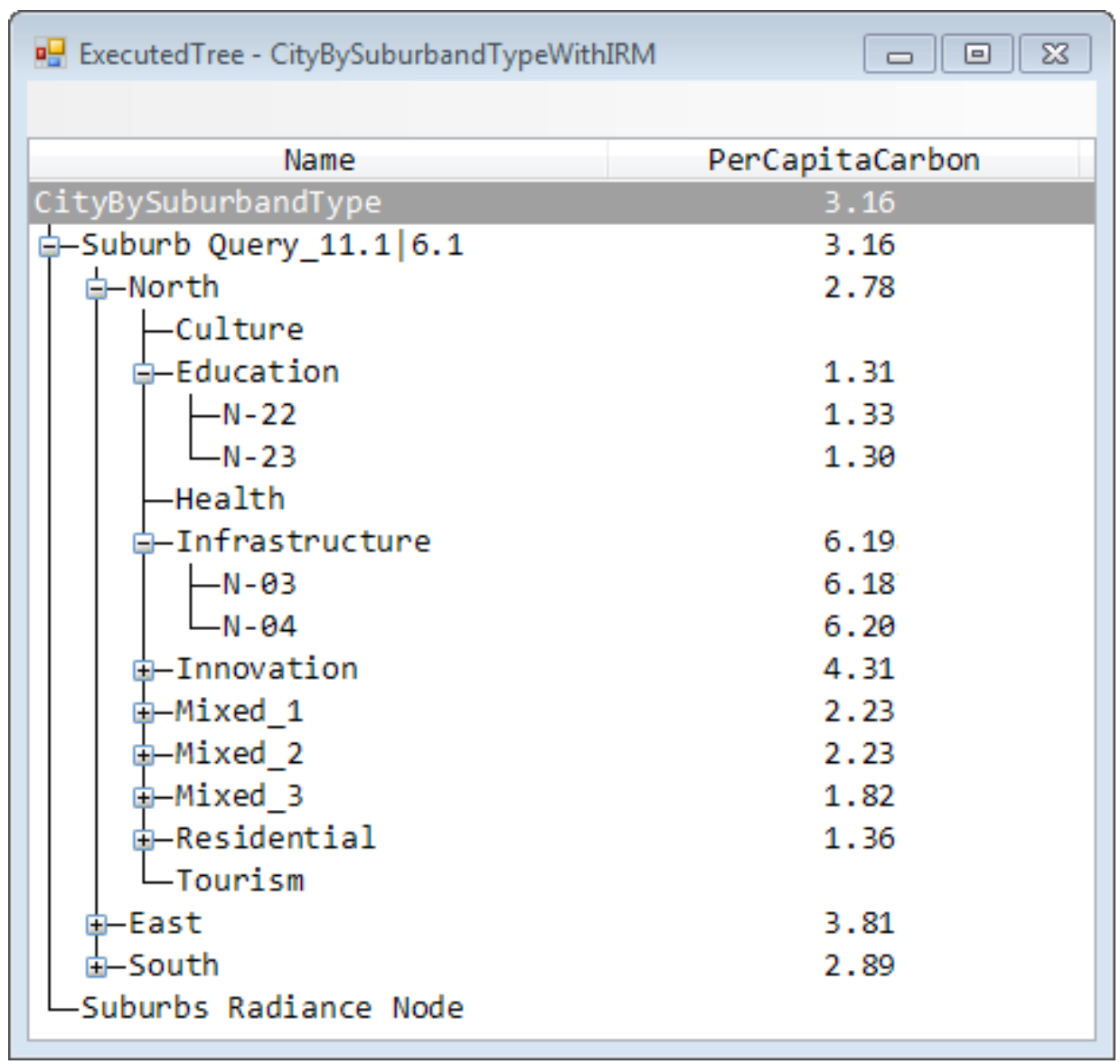

Figure 3.9: An executed HierSynth tree showing the percapita carbon KPI aggregated at all levels of the design decomposition. This tree is shown after execution, the template for the tree is shown in Fig. 3.7 (p99).

example an analysis to be carried out on all buildings in a suburb.

The ability for a template to filter the records it accepts enables different templates to be used for different design elements returned by the same parent query node (e.g. different analysis engines used on different forms of building).

All instances have a unique identifier formed by the template ID and the instance number (e.g. "15.1", "15.2" and so forth). Since queries may be nested this scheme is not guaranteed to produce unique identifiers. The solution to this is to concatenate the identifiers produced at each level of the tree such that a node might have an identifier of "1.1|2.1|3.4". This is somewhat analogous to the Dewey Decimal system. 
Listing 3.1: Pseudocode of the algorithm for expanding a query node.

1 public void ExpandNode(Node query, Dictionary<string, variablemap> scenarios) \{

\section{// execute queries}

Dictionary<scenario, List<record>> queryResults;

foreach (scenario in scenarios) \{

queryResults.Add (scenario, query.ExtractRecords (scenario);

\}

// process nodes not filled by their parent.

Node[] notFilledbyQuery = query. Children. Where(

child $=>$ !child.FilledbyParentQuery)

foreach(child in notFilledbyQuery) \{

processchild(child);

\}

// find all templates the query node will fill

Node[] filledbyQuery = query. Children. Where (

child => child.FilledbyParentQuery);

// for each child template filter records and group by record key.

foreach (template in filledbyQuery) \{

Dictionary<recordKey, Dictionary<scenario, List<record>>> templateInstances;

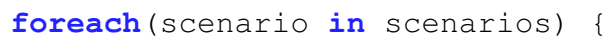

// finally recurse 


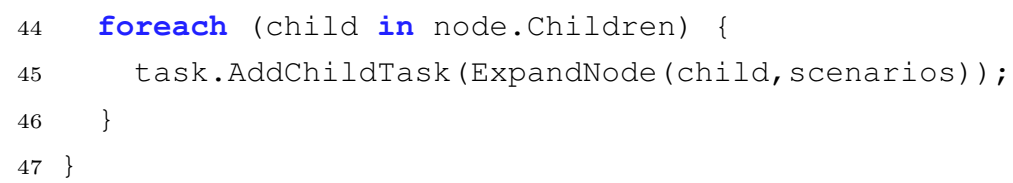

Fig. 3.9 (p103) shows the effect of expanding the tree shown in Fig. 3.7 (p99). We see that first the suburb query has queried a spreadsheet table and found three suburbs producing three sub-trees. Next the Building Type query has been executed in each suburb sub-tree and in each case returned a list of building types and instanced the building query and analysis tree for each of these. Finally the building query has been executed for each building type in each suburb and has in each case been parameterised by the scenario data which has been patched by the chain of nodes from the root node down to its parent node. In this case, two patches occur that affect the building query: firstly the suburb node will provide the suburb name to search within and then secondly the building type node will specify which type of building to search for. This specific query is then performed and the returning buildings of a particular type in a particular suburb and the combined building template and analysis node is instanced for each, thus producing the leaf nodes shown in the expanded tree.

Finally we should consider how this expansion phase occurs when executing a HierSynth tree under multiple scenarios. Whilst we could simply generate multiple separate trees it would be more useful to store multiple analyses of the same design element on the same tree node. However this can be made difficult since the shape of the tree can be different under each scenario, for example if each scenario is a different design there may be a different number of suburbs and buildings. Thus each template node must identify each record it processes by a primary key which is then used to match up records in new scenarios with previously generated child nodes (see line 28 of Listing 3.1 (p104)). This avoids duplication and enables a single data structure to store multiple sets of design and analysis data ready for the execution of various analyses.

\subsubsection{Execute Phase}

The execution of a HierSynth tree centres upon the analysis nodes it contains. These will be at various levels of the tree and so will analyse the design on various scales, perhaps at city wide or building level. These analysis nodes will take the design and scenario data now stored in the HierSynth tree to analyse one or more design elements in the tree represented by tree nodes. The analysis node will then prepare input for, run and extract results from external analysis tools. These analysis results will then be stored within the design element tree nodes.

As a proof of concept HierSynth has three types of analysis node implemented:

1. Radiance - is an open source lighting simulator in wide use in industry. See 
section 4.4.1 (p144) for more information.

2. Excel - is very widely used within the design and analysis communities for design analysis ranging from back of the envelope calculation to 50,000 cell multidiscipline analysis engines. Examples of these models can be found in section 4.4 (p144).

3. SynCity [53] - is an academic urban energy simulator comprising models for optimising an urban resource generation and interconversion network along with an agent based transport model.

How these nodes work internally is independent of the HierSynth data model, provided they meet criteria for accessing design, scenario and previous analysis results and store their own analysis results correctly. Implementations of new node types must inherit from a basic template node. Commonly these analysis nodes work by gathering data, formatting and presenting it to the analysis engine which is then initialised and run before the analysis results are read, post processed and stored in the HierSynth tree.

For example the Radiance node will either generate a $3 \mathrm{~d}$ model of the development from GIS data or use an existing model, then convert it to the right format for radiance analysis. Details of the analysis configuration (longitude and latitude, material types and time of day) will then be read from the analysis scenario and a simulation prepared. Radiance will then be initialised and run. This will produce a texture encoding daylight measurements. These are post-processed to compute averages for the roofs and fascades of a given area which are stored in the design elements node.

We will now consider where the analysis nodes access their data from. Execution nodes can access four types of data:

1. Node Data - if the analysis node has been used to represent a design element in the tree (as with the building nodes in Fig. 3.9 (p103)) it will likely have been generated by a query node and so much of the information required for the analysis will have been returned by the query and stored in the generated analysis node. Analysis nodes may also contain local configuration data which is not visible to other nodes.

2. Scenario Data - this dictionary of variables and values will contain some configuration data such as the weather of the development. The scenario variable map will have been patched successively by each ancestor node of the analysis node from the root node down to the parent node and finally with the data of the current node. These patches will have added information about the hierarchy of design elements that the current node sits under and will be different for each analysis node depending upon its location in the HierSynth tree. 
3. HierSynth Tree - Design and scenario data from other nodes can be read freely by analysis nodes providing it is not written to. This enables access to, for example, the set of all electricity substations in the design to enable an analysis of whether they cover the development effectively.

4. Analysis Results - Analysis results from other nodes may also be accessed provided these links are stated clearly before execution. These Execution links will be discussed shortly.

Having gathered the required information, the analysis node transforms it for use by its analysis engine (e.g. a lighting analysis package). If needed temporary folders are created based on the tree structure and scenario name; results are then stored in this directory. The analysis engine is then started (subject to resource contention described in section 3.12 .2 (p124)).

Finally once the analysis engine has finished results are post processed and stored either in the analysis node itself or potentially upon other design element nodes. Large analysis files may be stored permanently in the results folder specific to that analysis run.

\section{Execution Flow}

As mentioned earlier the order of expansion of the HierSynth tree is from root node to leaf node since this enables more detailed queries to use results of higher level queries (buildings in a particular suburb). However the execution process works from leaf node to root node. This is so that higher level analyses can use the results of lower level analyses. For example a district-wide energy system optimisation run from the SynCity module might use results from an energy demand module run upon each building.

As just discussed, if one analysis node requires results from another then HierSynth will order the analyses to occur in the required order. This implies that rather than a tree of execution tasks we have a Directed Acyclic Graph (DAG) of execution tasks. We note that the execution order from leafs upward means that one node waiting for another will cause it to wait until that node's descendants have also been executed.

The execution of this DAG is achieved by using a worklist algorithm implemented by an underlying task parallel framework in the .Net runtime. The HierSynth data model also affords the opportunity to ensure the execution graph is a DAG. Dependencies between nodes are either data-flow driven (i.e. using data from an ancestor or child node) or alternately by an explicit execution link defined by the user. In the first case the dependency could be checked during tree compilation since all data dependencies within a node can be extracted using the expression language described in section 3.6.2 (p97). Similarly since execution links are user defined these could be identified, checked to ensure a DAG and visualised to the user alongside data dependencies. 


\subsubsection{Aggregate Phase}

Having executed the analyses one means of generating insight into the design is to compute metrics across all design elements. This is done by an aggregation pass which starts from leaf nodes works up to parent nodes aggregating metrics from child design elements. A simple example is calculating the average percapita carbon emissions for a set of buildings or a suburb. This is shown in Fig. 3.9 (p103) where we see the analysis results on the building level and then aggregate results on the building type, suburb and city levels.

These metrics allow quick comparison of groups of design elements and enable quick drill-down into results to identify problem design elements - for example the East development is performing poorly compared to its peers.

To compute these metrics a set of "Datalayers" are set up and computed over the tree. They use the internal mathematical expression language [85] to enable custom logic in the aggregation process. For example whether or not to accept a node or child node result, the initial seed value for aggregation, an aggregator function and a final processing function are all customisable expressions which can use any analysis result on the node or its scenario. As an example this expression language could be used to construct a percapita carbon figure across all districts in a development which is weighted for population.

These "Datalayers" then form the basis for building performance trees across the tree of design components as a visual tool for identifying performance problems across many KPI's and disciplines.

A more complex requirement might be calculating the total carbon emissions from multiple sources, first within a single node and secondly across the entire design decomposition. For this a set of data-layers would be utilised to create a tree like hierarchy of summations across multiple disciplines (e.g. transport and electricity consumption) resulting in a final root total. This, in essence is a performance tree; the design of which we will now discuss.

\subsection{Performance Trees}

As discussed in section 2.7.3 (p69) a performance tree is a visual indicator of the performance of a design element. It consists of a tree containing many KPI analysis results which form the leaf nodes of the tree. These KPI's are then combined to give a series of scores at each branch node of the tree. Finally an overall score for the design element is generated at the root node of the tree. Performance trees enable the composition of multiple KPI's from multiple disciplines into a single structure to give an overview of the performance of the design element. This overview gives not only a composite score to the design element which may be compared to its peers but enables 
quick visual investigation of performance problems by drilling down the performance tree. For example a poor score may result from a single KPI in one discipline being sub-par. This would be highlighted in the chain of parent nodes from that KPI to the root node which should reflect a poor score. A conceptual model of the construction of such a performance tree from many KPI's and disciplines is shown in Fig. 3.10 (p110).

A good performance tree should have a mix of KPI's from analysis results as well a good hierarchy of composite scores combining disparate KPI's. There are many means of computing these scores from a simple sum or average through weighted averaging to more advanced methods such as neural preference trees [25]. These aggregators must be set up to convert the disparate units of the KPI's into a utility score in a way which reflects the priorities of the engineers and designers. For example if the client would like to increase rental space then this should be highly weighted, on the other hand if the building will break planning laws even by a small amount this should be flagged up heavily through the performance tree to the root node. The main difficulty in using performance trees is setting up the aggregators in a meaningful way. Integrating formal methods of creating and aggregating metrics may be one means of achieving this (e.g. [86]). Alternatively one could explore the use of machine learning techniques such as [73] to construct performance trees automatically using Inductive Logic Programming to synthesise logical formulas representing a practitioners assessment of a training set of buildings. Recent research into learning multi-clause logical formulas will map simply to a performance tree concept.

Fig. 3.11 (p111) is a HierSynth-generated performance tree. HierSynth uses the aggregation phase of execution to compute multiple datalayers across the HierSynth tree. These datalayers are able to reference one another in the computation of results (e.g. adding $\mathrm{KwH}$ of electricity and gas consumption). These references between datalayers are automatically identified via the expression language used in HierSynth and used to create the performance trees shown in this figure. Performance trees are generated for each node in the HierSynth tree (see 3.12 (p112)). This enables comparison of the performance of (and designer preference to), for example, all the buildings in a development. To enable this visually each node of the performance tree is shown on a colour scale in comparison to its peers in all of the performance trees of other nodes in order to highlight comparisons. Since datalayers can access their corresponding results on child nodes the performance tree of a parent node can be computed using results from child design elements and analyses. This is shown in the conceptual model shown in Fig. 3.12 (p112). This provides a useful facility to for example rate a suburb on the distribution of the scores of its child districts. This in turn is possible since the data layers involved at each node need not be the same, enabling different performance trees for each node in the HierSynth tree. Similarly different sets of data layers can be used to generate different performance aspects which are of interest to different stake holders. These 


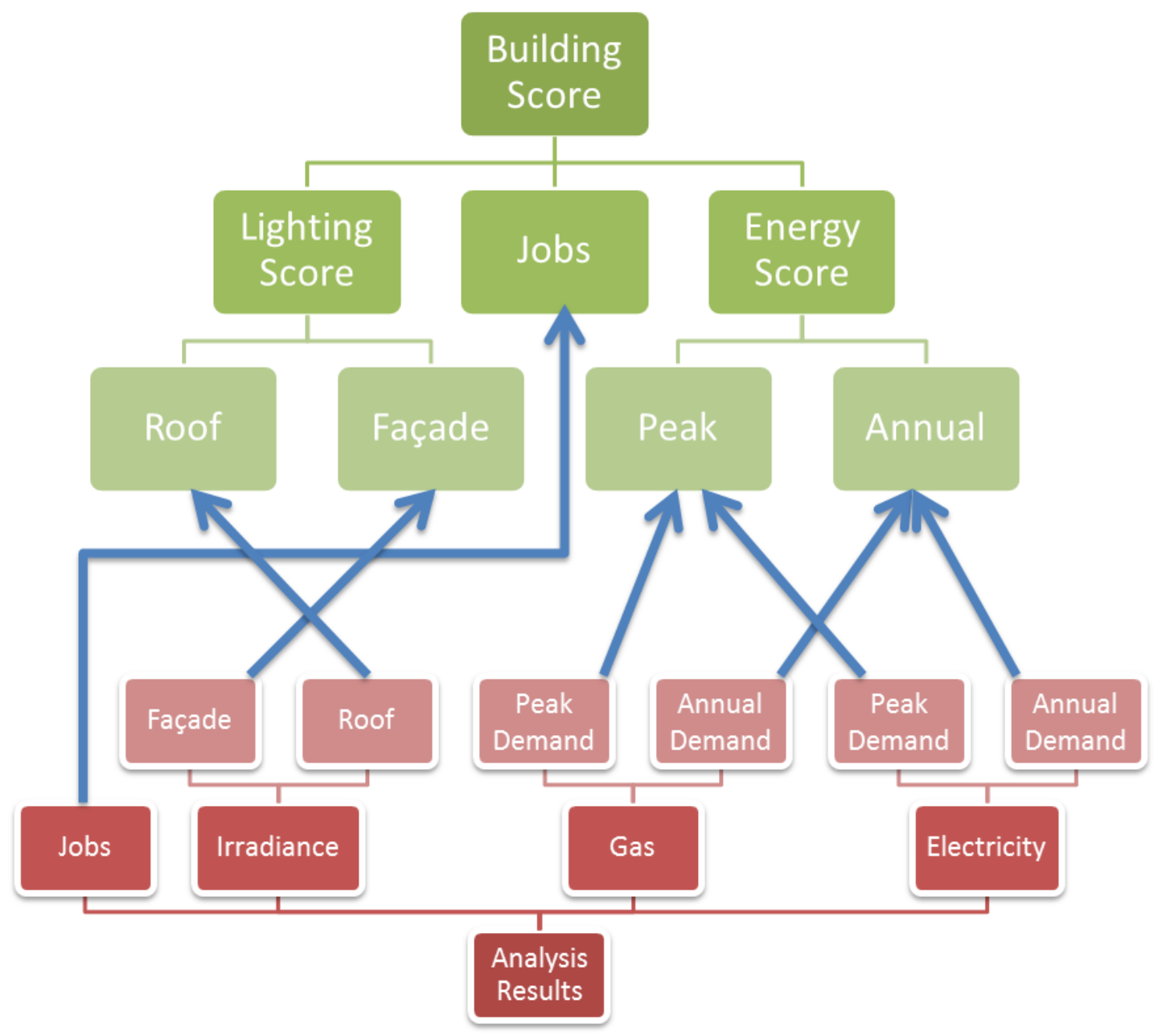

Figure 3.10: A conceptual model of a performance tree for a design component in HierSynth. The tree is based upon analysis results stored in that node (shown in red and as a tree due to the naming convention used in HierSynth as described in Fig. 3.6 (p97)). 


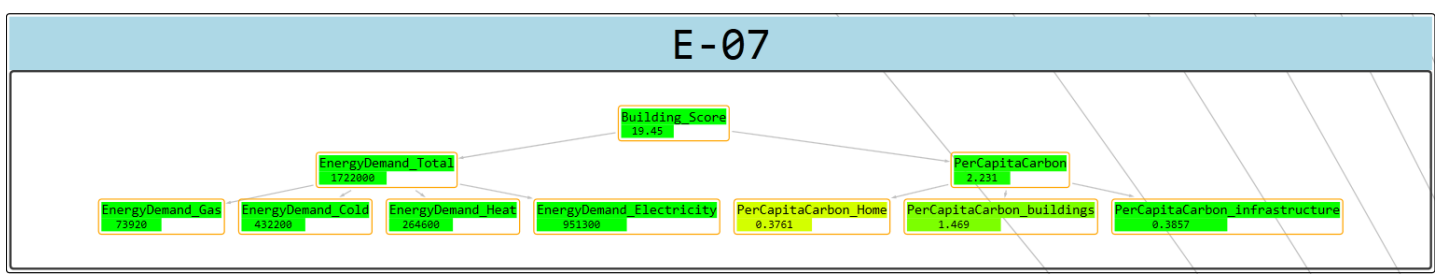

Figure 3.11: A Performance tree calculated by HierSynth displaying KPI's from a number of disciplines and aggregating them into composite scores for each area and for the whole building. Colour coding is by performance in relation to peers and enables a quick visual diagnostic of building performance. The perceptive reader will notice lines in the background which are references between multiple performance trees in the design decomposition; this performance tree is just one of around 100 performance trees in a design decomposition breaking a development down into suburbs and districts. Fig. 3.12 (p112) shows this concept.

can then be visualised geolocated in $3 \mathrm{~d}$ as is shown in section 4.11 (p166). Finally the most important use for these performance trees is comparing design performance at all levels under different scenarios, be they analysis scenarios or different designs.

\subsection{Graph Structure}

In this section we discuss how the HierSynth data model supports a richer structure of analysis model integration using cross links within the tree structure to regain much of the flexibility lost when restricting the user to a tree based hierarchy. We first consider how such cross links are implemented and then motivate their introduction with an example which requires cross links to enable multi-scale analysis.

\subsubsection{Execution Links}

One of the disadvantages to adopting a tree structure for the data model of HierSynth is that it means that the richer connectivity of a more traditional graph based workflow model are lost. This means it is difficult to implement multi-scale analysis where a high-level analysis must refer to the results of a lower scale analysis. To address this HierSynth uses a concept of "Execution Links" to add cross-structure to the tree and regain much of the expressibility of a graph based workflow layout.

Any node within HierSynth is able to provide a list of execution links. These are references to other nodes within the hierarchy. Upon execution the processing of a node is delayed until all of the nodes referenced via execution links have been executed. The data from referenced nodes is then made available to the execution of the node via prefixed scenarios (i.e. the names of all outputs of a node are prefixed with an identifier). Given the execution semantics (with execution running from leaf to root) 


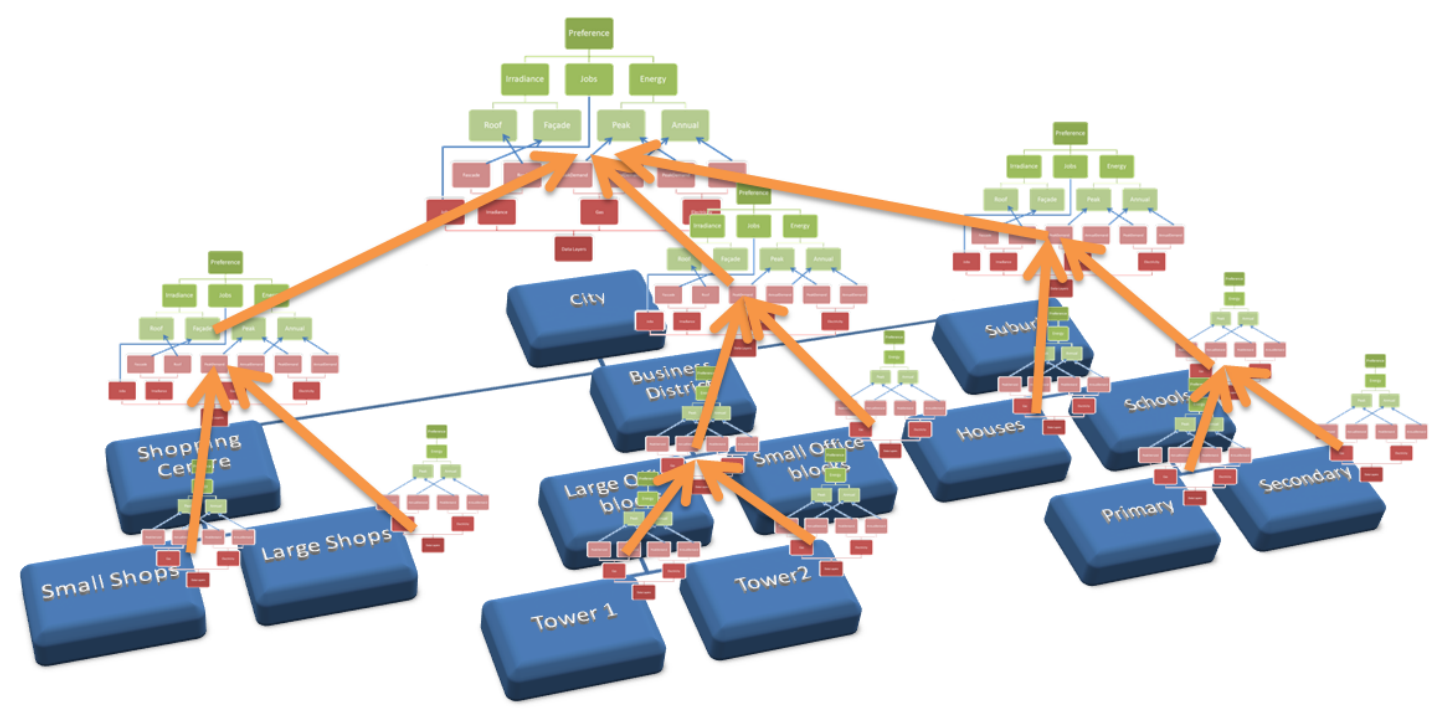

Figure 3.12: Conceptual diagram showing the formation of the performance trees for each design component. Each design component's performance tree is formed from KPI's stored on that node (in red) together with the corresponding performance tree nodes in the design component's children. This allows quick drill-down of performance results both in the performance tree, across disciplines and through the design decomposition.

of a HierSynth tree a node can only specify execution links to its descendant nodes, its sibling nodes or their descendants. Should this limit a model's use the opposite execution order (root to leaf) could be used to enable links to parent nodes (but this would preclude links to child nodes). These semantics enable references from a high level to a lower level analysis, however many high level analyses will require access to a large set of lower level analyses. To enable this, every execution link made will not only allow access to the referred node's data but also the node itself. Given the execution semantics (leaf to root) we can guarantee that all descendants of the referred to node will have been executed. Thus the high-level analysis is enabled to query and access data from all descendant nodes of a node it has an execution link to. This would include searching for all lower level analysis nodes and their analysis results.

Using this scheme multi-scale analysis is enabled with models at a higher scale referring to those at lower levels. We now motivate this with an example.

\subsubsection{SynCity Analysis Example}

To motivate this functionality we explore a case study where a high-level city-wide analysis requires inputs from a lower-level building level analysis. Specifically a citywide power network optimisation model (SynCity) requires energy demand forecasts for each building which are calculated using an Integrated Resource Management model (IRM model). 


\section{SynCity Model}

SynCity is a tool for "integrated modelling of urban energy systems" [53] developed by the Urban Energy Systems project at Imperial College London. It is one of the first projects to integrate several layers of modelling into one tool to enable crossoptimisation of a cities' total energy consumption across multiple disciplines (masterplanning and various forms of energy generation networks and inter-conversion technologies). Specifically it integrates a city layout optimisation, an agent based energy usage simulation and a resource interchange network which will meet the predicted demand in an optimal method with specified technologies. The framework has been applied in numerous case studies in the UK and abroad, exploring differing methods of energy supply, city layout and carbon reduction strategies demonstrating that the model can be "used to develop alternative master plans for a new development with up to $90 \%$ reduction in cost and emissions against a business-as-usual scenario".

The model we consider in this example is the third in the SynCity model stack. This model creates an optimised Resource Technology Network (RTN) which aims to meet energy demand forecasts for various resources (heat, cooling and electricity) with various resources (e.g. Gas and Biomass) and power technology (e.g. Combined Heat and Power plants) in an optimal and spatially consistent manner. This is implemented as a Mixed Integer Linear Programming problem aiming to minimise cost and various types of pollutant emission. A number of different technologies are specified for use in this model ranging from combined heat and power plants to biomass energy generation. These technologies can be interconnected as required for an optimal solution.

\section{Implementation}

As shown in Fig. 3.13 (p114) this analysis tree consists of a city design decomposition into suburbs, building types and buildings. Annotated onto this decomposition are per building runs of the IRM model which will calculate demands for electricity, heating and cooling at a granular level. At the city level the SynCity RTN model will be run. This node has an execution link to the "Suburb Query" which is its sibling. Since the execution order runs from leaves to root when the SynCity analysis runs all of the building level analysis will have been completed and the SynCity model will be free to query the "Suburb Query" node for its descendant IRM analysis nodes. From these the forecasts for heating, cooling and electricity can be extracted and entered into the SynCity model. The results of the analysis are visualised in Fig. 3.14 (p115).

This multi-scale analysis is only possible through the execution links interface together with the execution semantics of the HierSynth tree. In this way much of the functionality lost by limiting the user to tree structure is regained.Execution links proved a critical feature for the integration of many analysis models together, as can 
Name

City

Tuburb Query

当-Building Type Query

当-Building Query

LBuilding IRM

Suburbs Radiance

SynCityRTN
Type

SimNode

ExcelQueryNode

ExcelQueryNode

GISQueryNode

ExcelNode

RadianceNode SynCity FourNode

Figure 3.13: This HierSynth tree makes use of cross-links between the SynCity model and the suburb query decomposition. This enables the high-level SynCity analysis to access the energy demand forecasts calculated by the Integrated Resource Management (IRM) model which is run for every building of every type in every suburb. The SynCity model then calculates an optimal resource network and set of power technology to meet these demands. This is shown in Fig. 3.14 (p115)

be seen in section 4.9 (p157).

\subsection{Execution Chains}

Having explored the design and working of a HierSynth tree along with its execution algorithm we can now explore how they are used within the HierSynth platform. Firstly as we have seen a HierSynth tree can be used to analyse a design, for example the city decomposed down by suburb and building shown in Fig. 3.9 (p103). Secondly they can be used as the building blocks of more complex in-depth analyses and investigations. For example analysing a design under multiple analysis scenarios (e.g. times of the year) or analysing a whole suite of designs. However the variable mappings for these scenarios must each be entered manually. This is something that can be addressed using an execution chain consisting of several HierSynth trees.

To investigate questions, such as how different development designs perform under several carbon reduction plans, it is useful to chain together several HierSynth trees. These trees perform different functions, such as the generation of scenarios (perhaps through simulation) and the analysis of the design. Data is passed between the different 


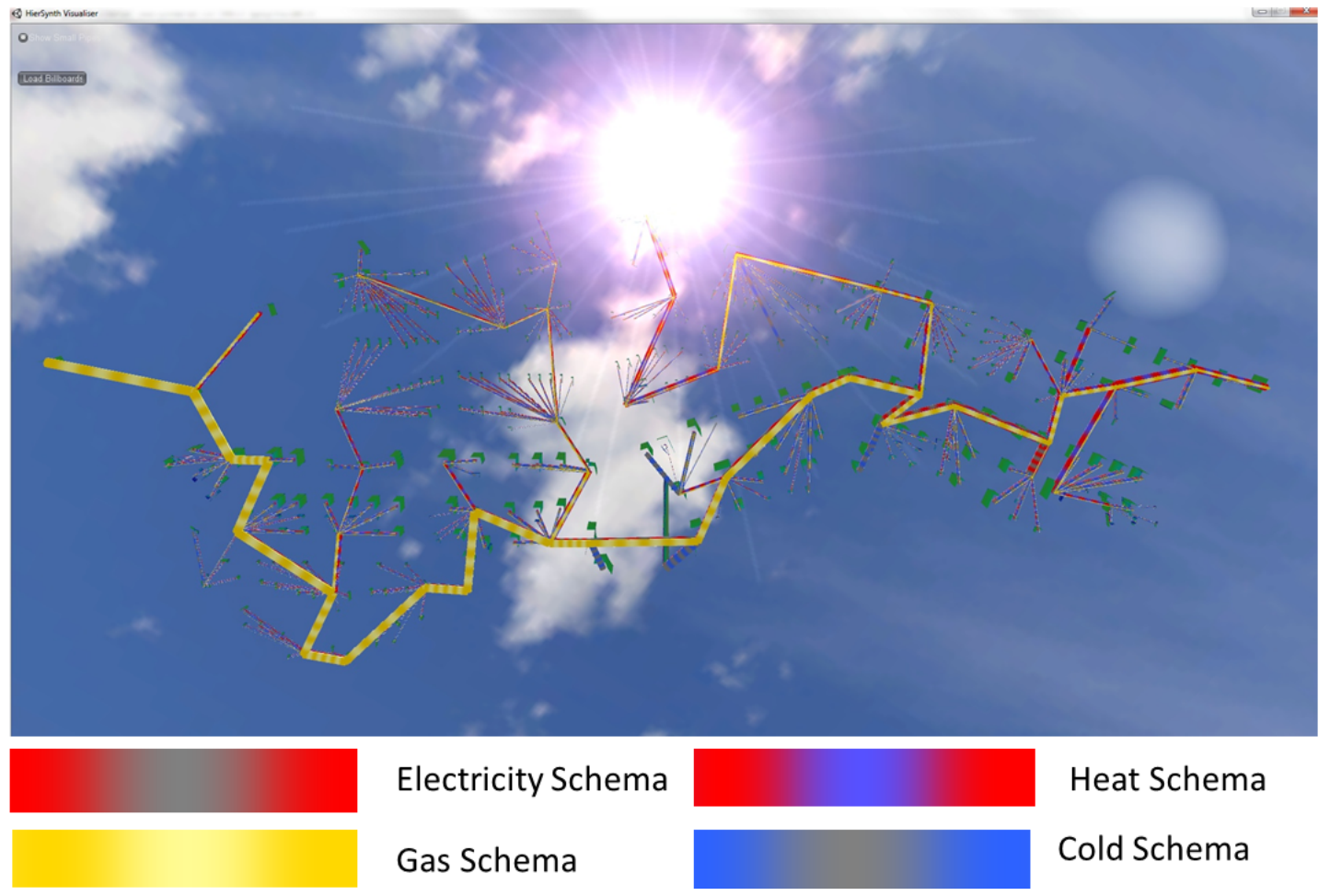

Figure 3.14: A "worms eye" visualisation of the output of a SynCity Resource Technology optimisation run. Four resource networks are visualised which are providing power to the city to meet the electricity, heating and cooling demands of the city. Interconversion technology (e.g. gas boilers or Combined Heat and Power (CHP) plants) are placed strategically within the city. 
trees in the execution chain by extracting different variable mappings (scenarios) from selected nodes of the previous tree and using them as scenarios to execute the next tree.

The benefits of using execution trees are shown through a number of example execution chains are shown in Table 3.1 (p118). These chains show the initial set of scenarios and then a number of HierSynth trees each executed for each of the scenarios generated by the previous tree. Several of these examples are taken from the casestudy in Chapter 4 (p139). First note that all four execution chains reuse the same HierSynth tree for analysing the development allowing direct comparisons between all scenarios explored and reducing implementation costs.

\section{Example A}

The first execution chain $(a)$ shown in Table 3.1 (p118) aims to investigate the performance of a set of designs which vary in density. Additionally there is a requirement to investigate the impact of several sets of carbon mitigation strategies upon each design. These impacts are then explored over time, as discretized into ten construction phases (each a few years long). The goal of this study is to help the design team understand the design trade-offs in terms of density and investment in carbon mitigation strategies and how these will vary over time.

The execution chain $(a)$ for this study shows the use of scenario composition to generate scenarios as will be discussed in the next section. Thus tree 1 produces the cartesian product of a set of designs and a set of carbon reduction strategies creating a set of scenarios for analyses. This is done for each of the ten initial construction phases (phases of development; the number of these will depend upon the project and is chosen here arbitrarily). Finally the second HierSynth tree is executed for 10 constructionphases $* x$ designs $* y$ carbonstrategies. This enables a great deal of detailed insight to be generated by comparing designs under different carbon scenarios over the time represented by the construction phases (an example of this is seen in Fig. 4.6 (p155)).

\section{Example B}

Another possible requirement for computational investigations is to consider the performance of a design under a predicted future climate. Such predictions might be made through the use of complex simulation models. Execution chain $b$ ) shows the use of simulation to generate analysis scenarios in this case possible future climate scenarios, perhaps computed using a lighting analysis and used in a building energy study where solar gain is important. 


\section{Example C}

One common use of computation within a design context is investigating the best value or the impact of a particular design parameter or analysis assumption. In this case we explore the impact of different uptakes of electric cars and study the impact upon different design densities under different carbon mitigation strategies.

Execution chain $c$ ) is similar to $a$ ) except that it operates for a single initial scenario and uses HierSynth generator nodes to create a set of scenarios which explore the changes in an analysis assumption at 10 different levels. In this case the uptake of electric cars which is explored in section 4.12 .1 (p172). This enables complex explorations of the design space to be set up in minutes.

\section{Example D}

A common design space exploration technique is sensitivity analysis. This enables the study of which inputs (design variables or analysis assumptions) a performance figure is more sensitive to changes in. Sensitivities will likely vary with different designs, under different scenarios (e.g. carbon mitigation strategies), hence a wide sweep of the design space performing several sensitivity analyses concurrently should provide a great deal of insight for practitioners.

Finally execution chains need not only consist of HierSynth trees; one very useful approach is to splice a sensitivity analysis into the execution chain $d$ ), first to generate the experimental runs, then again after the analysis modules of interest are executed to compute sensitivities to various factors in the scenario. This will be discussed in more detail in section 3.14 (p127) and an example tree is shown in Fig. 3.18 (p130).

Fig 3.2 (p117) shows a specification of the grammar for the composition of HierSynth trees into a execution chains in Extended Backus-Naur Form (EBNF). We see three options. Firstly, a chain of scenario generation trees, constructing a multi-dimensioned exploration of the design space followed by a final analysis tree. Secondly, we see a sensitivity analysis spliced before and after a final analysis tree. These steps create and then analyse the set of experimental runs to be performed. Finally, we see an, as yet unimplemented, proposal for how an optimisation loop could be included in an execution chain. In this case the optimiser would normally be a genetic algorithm with each scenario being a different design variant. The optimiser would create a new generation of design specifications. Scenario generation trees would then be used to turn these specifications into actual design geometry which would then be analysed by an analysis tree, completing the analysis loop. Both of these last two options are optionally preceded by a number of scenario generation trees to create larger design space explorations. 


\begin{tabular}{|c|c|}
\hline $\begin{array}{l}\text { a) Scenario Generation and Anal- } \\
\text { ysis tree see Fig. } 3.16(\mathrm{p} 121)\end{array}$ & $\begin{array}{l}\text { b) Scenario Generation through } \\
\text { Analysis }\end{array}$ \\
\hline $\begin{array}{l}\text { 0. a) Ten construction phases } \\
\text { 1. }[\downarrow] \text { Compose Designs with Car- } \\
\text { bon Strategies } \\
\text { 2. }[\uparrow] \text { Analyse Design }\end{array}$ & $\begin{array}{l}\text { 0. b) Initial Scenario } \\
\text { 1. [ }[\downarrow] \text { Simulate } 5 \text { Climates } \\
\text { 2. [个] Analyse Design }\end{array}$ \\
\hline $\begin{array}{l}\text { c) Electric Car Investigation see } \\
\text { Fig. } 4.17(\mathrm{p} 173)\end{array}$ & $\begin{array}{l}\text { d) Sensitivity Analysis see Fig. } \\
3.18(\mathrm{p} 130)\end{array}$ \\
\hline $\begin{array}{l}\text { 0. c) Initial Scenario } \\
\text { 1. [ } \downarrow \text { ] Compose Designs with Car- } \\
\text { bon Strategies } \\
\text { 2. [ }[\text { ] Generate levels of electric car } \\
\text { uptake } \\
\text { 3. [个] Analyse Design }\end{array}$ & $\begin{array}{l}\text { 0. d) Initial Scenario } \\
\text { 1. [ } \downarrow \text { ] Compose Designs with Car- } \\
\text { bon Strategies } \\
\text { 2. (*) Generate Sensitivity Analysis } \\
\text { Experiments } \\
\text { 3. }[\uparrow] \text { Analyse Design } \\
\text { 4. (*) Analyse Sensitivity Analysis } \\
\text { Experiments }\end{array}$ \\
\hline
\end{tabular}

Table 3.1: Example HierSynth execution chains composed of a number of HierSynth trees. Some HierSynth trees are used to generate analysis scenarios and these are discussed in section 3.11 (p120). 


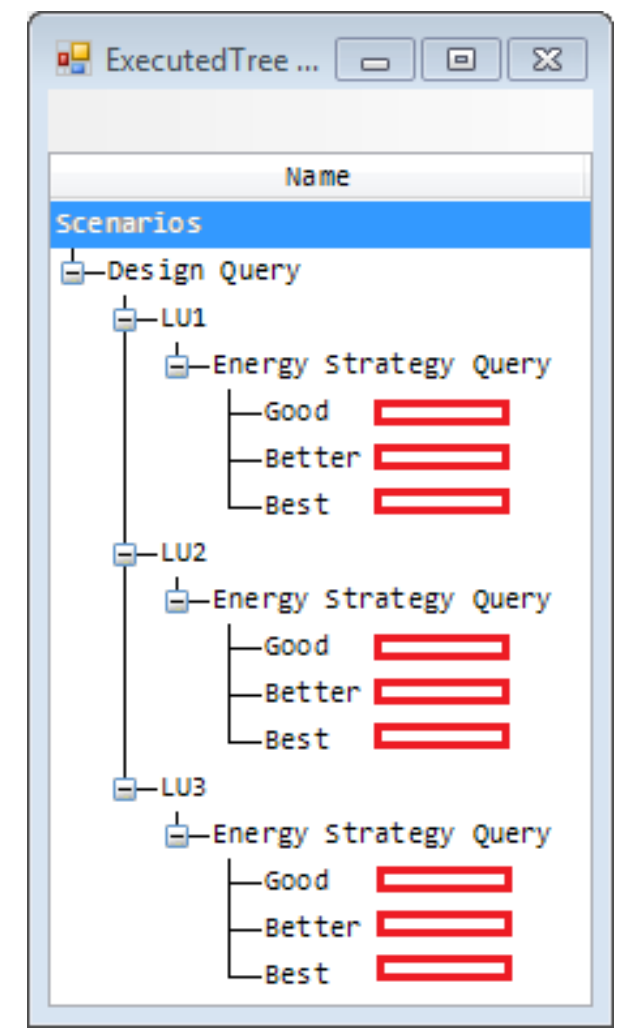

Figure 3.15: An example of an executed scenario generation tree. Note that in this case only the leaf nodes will generate scenarios from their variable mappings which will have been patched to include energy scenario data and by their ancestor design node to include design data.

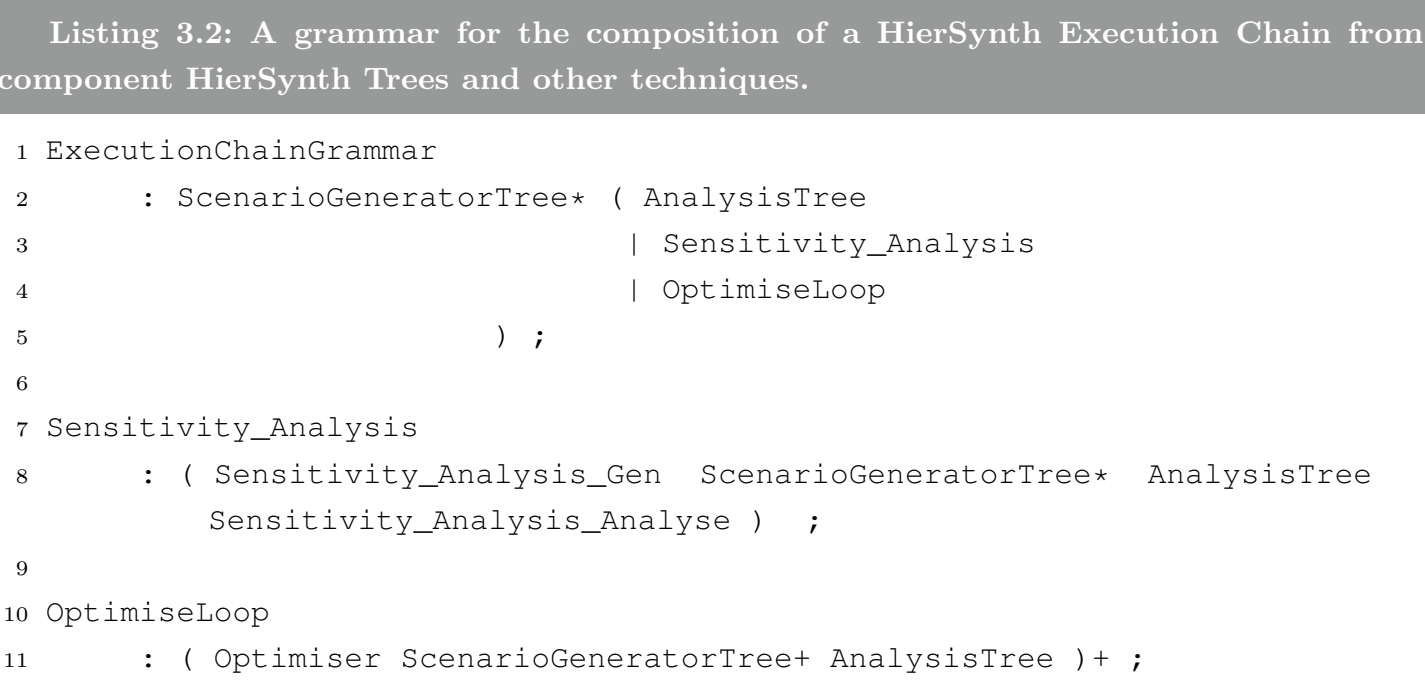

Thus execution chains provide a key means for investigating a design and its design space. As we have seen this involves using HierSynth trees to generate scenarios, the mechanics of which we will now discuss. 


\subsection{Scenario Generation}

Scenario generation enables many opportunities for creating design insight. Scenario generation uses a standard HierSynth tree consisting of query, analysis or template nodes to produce new analysis scenarios. New scenarios are extracted from the variable mappings on certain nodes (normally the leaf nodes). To enable this, a change is required in the tree execution algorithm. Under normal execution a HierSynth tree is executed from the leaf nodes upward to enable parent nodes to use child node analysis results. However if a HierSynth tree is tagged to be executed as a scenario generator the execution is completed from the root node downward. This enables child nodes to execute in light of their parent node's results. This in turn enables the node to patch the variable map with analysis results.

Consider the example executed tree shown in Fig. 3.15 (p119); here we see the first tree in execution chain $a$ ) of table 3.1 (p118). In this tree the goal is to generate scenarios to analyse a number of designs under a number of energy scenarios. When expanded, the two nested queries will be evaluated, instancing a set of design nodes and for each of these a set of energy scenario nodes.

When the execution phase begins it will do so from the root node and we follow the changes made to the variable map down through the tree. Each design instance node will use data returned by its parent query such as the design name to read in full design information from external files (e.g. from a land use schedule). This information will be added to its variable map at that node. Next each energy strategy node will execute and query its information from external data files again adding it to the variable map.

Finally when execution is complete the tree will be searched for nodes which are tagged as producing new scenarios. In this case the leaf nodes of the tree (highlighted in Fig. 3.15 (p119)) and their variable mappings will be extracted as new scenarios. Recall these will consist of the original scenario patched with information about a specific design and a specific energy scenario.

This process is completed for each of the scenarios in the execution chain. For example the 10 construction phases in chain a) of Fig. 3.1 (p118) would each produce 9 design / energy strategy scenarios. This results in the 3 designs being analysed under 30 scenarios covering a time and an energy strategy dimension.

\subsection{Performance}

Given the mismatch in speed between the design and the analysis cycles attention should be focused upon increasing the speed of analysis. To this end much effort was spent upon the performance of the HierSynth framework. This was achieved through parallelisation, caching and application sharing within the HierSynth framework and 


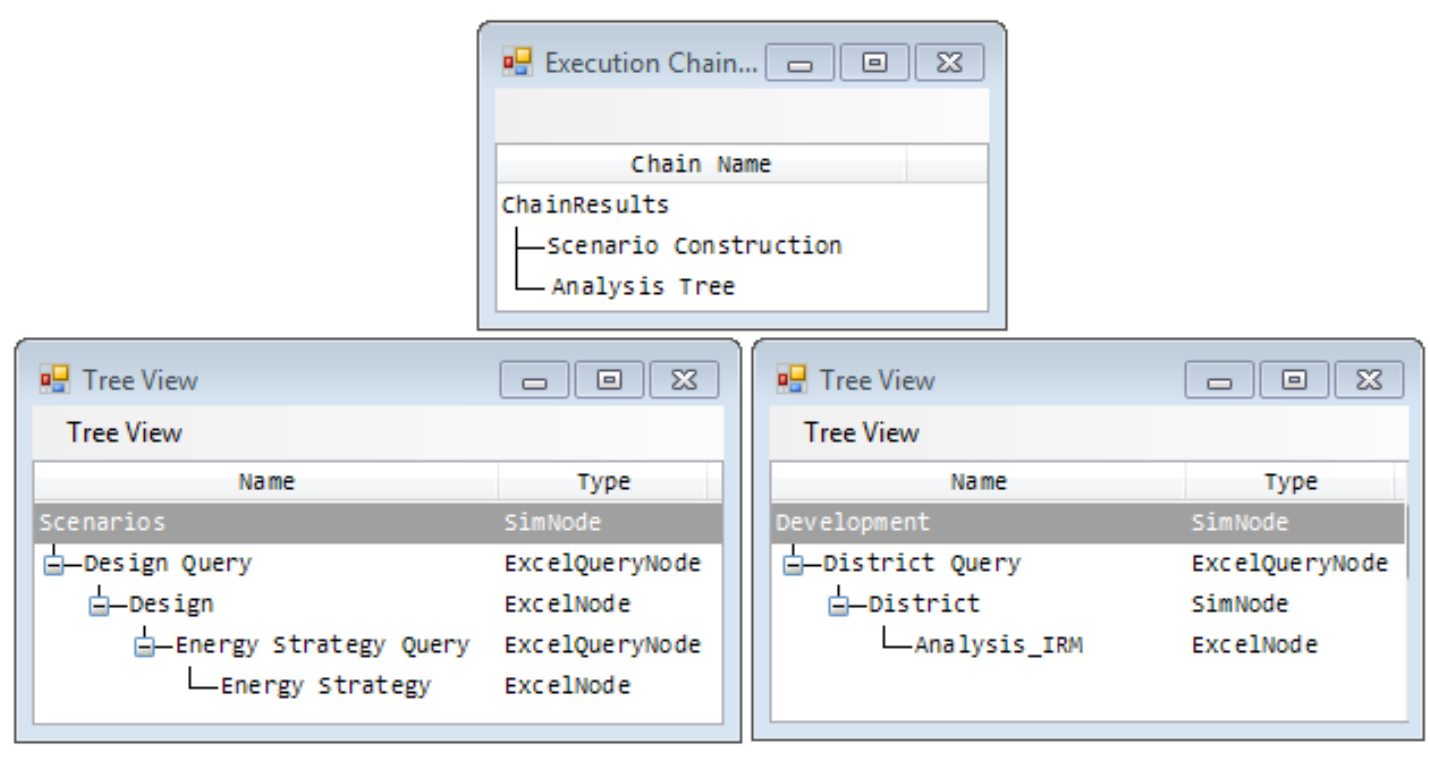
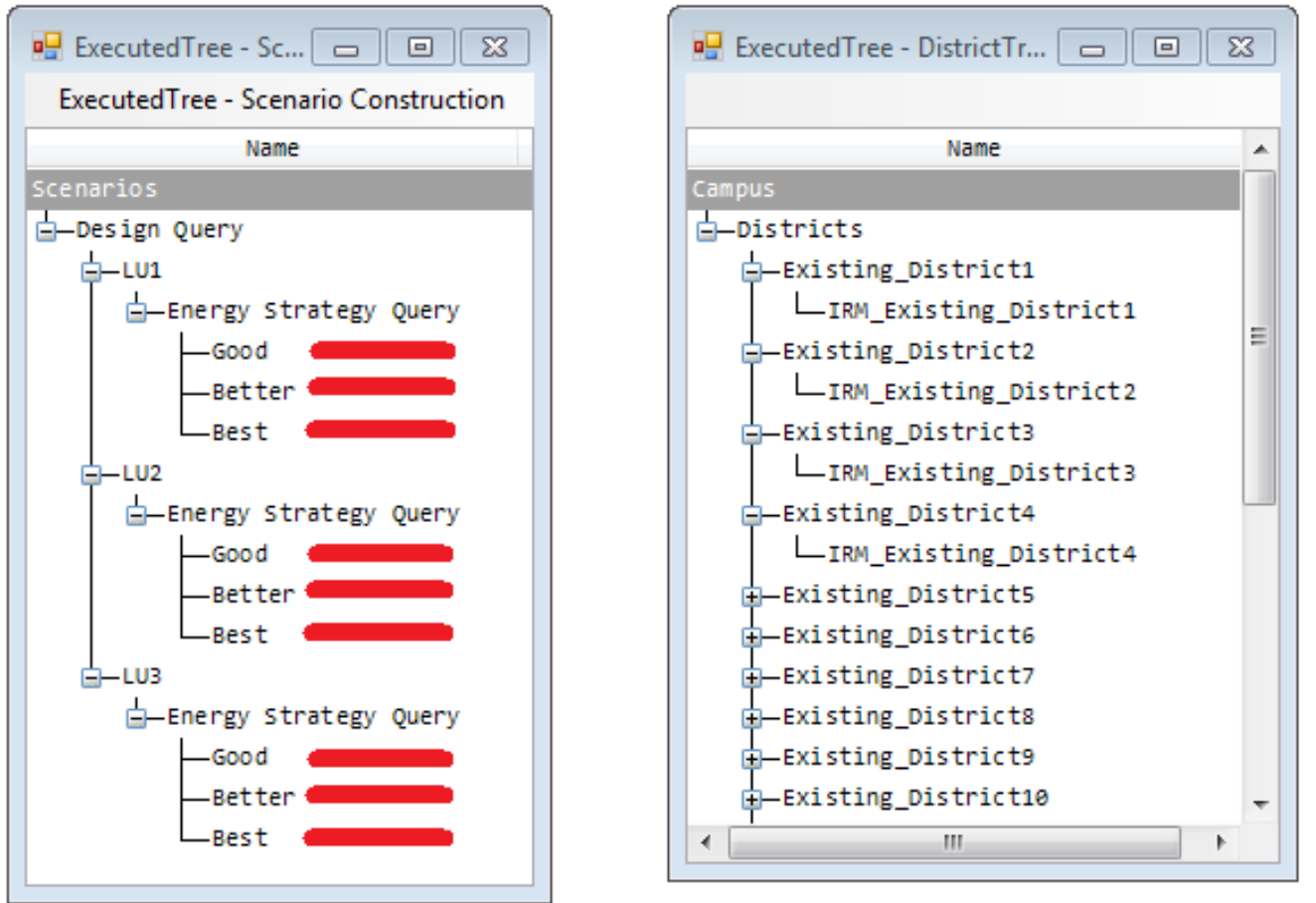

Figure 3.16: A simple Execution Chain consisting of a scenario generation tree and an analysis tree. The first generates the composition of designs and energy strategies as 9 new scenarios and the second analyses the design under the scenario with a per-district design decomposition. 
enabled the framework to manage thousands of connected analyses. HierSynth was able to return results in 5-90 minutes depending upon analysis run complexity. This should be compared to the previously manual processes and as such is a substantial improvement over analyses which would have taken hours of manual work. From the case study in Chapter 4 (p139) we saw an order of magnitude more analysis cycles being undertaken through the project than literature would suggest is normal.

First however a word about the analysis engines HierSynth automated. We did not explicitly change the actual analysis models but rather added code to automate the preparation of input, manage analysis execution and handle post processing of output. Analysis engine execution occurred locally (as in the case of Radiance [103] a lighting simulator) or remotely (as in the case of the SynCity [53] models). Some analyses permitted concurrent execution (e.g. Excel models) whilst others allowed only one simulation at a time (e.g. SynCity). For example the Excel analysis module was able to parallelise executions for up to 5 concurrent executions on a quad core (Intel i7 720QM) laptop. This parallelisation gave most scope for increased performance and was enabled via the HierSynth tree based data model.

\subsubsection{Parallelisation}

To take advantage of compute resources and to overlap long running analyses HierSynth takes advantage of the hierarchical decomposition of the design and uses a task based execution to provide tree based parallelism. For each of the three stages of execution discussed in section 3.7 (p100) (expansion, execution and aggregation) a task hierarchy is created to reflect the HierSynth tree. These trees are dynamically expanded both as the expansion phase executes and the HierSynth tree grows and as downward execution and aggregation completes node by node as child tasks are created and executed. These three trees are joined as the children of the execution task which manages the execution of a HierSynth tree. This large tree is then augmented with other tasks such as checkpointing of results. A visual reflection of this task tree is then displayed to the user via a graph based visualisation as can be seen in Fig. 3.17 (p126) enabling visual feedback of task completion and growth of the execution tasks reflecting the HierSynth tree.

Each task in the execution tree has the option to execute its children either sequentially or in parallel. This is set through a design time switch. This choice, made at design time, is made depending upon the nodes task; the default is for parallel execution with the majority of nodes executing their children in parallel. Tasks such as the root task will execute their children sequentially to ensure the correct order of expansion, execution and aggregation. This enables a large amount of parallelism. The number of parallel tasks available at any point will depend upon the design decomposition implemented by the user. For example a decomposition of a design into suburbs and buildings will result in many potentially parallel tasks whilst a decomposition into 


\begin{tabular}{|c|c|}
\hline Parallelisation Opportunities & Parallelisation Challenges \\
\hline $\begin{array}{l}\text { - }[\checkmark] \text { Scenario Preparation } \\
\text { - }[\checkmark] \text { Each Design Decomposition } \\
\text { Stage } \\
\text { - }[\times] \text { Multiple Analysis Scenarios } \\
\text { - }[\times] \text { Overlapping Execution Phases }\end{array}$ & $\begin{array}{l}\text { - Computational Resources } \\
\text { - Analysis Engine Supports Multiple } \\
\text { Instances } \\
\text { - Analysis Engine Licensing } \\
\text { - Dependencies between Execution } \\
\text { Tasks }\end{array}$ \\
\hline
\end{tabular}

Table 3.2: HierSynth parallelisation opportunities and challenges within the HierSynth tree execution algorithms, along with current implementation.

suburbs will not. The realisation of this parallelisation will depend on a number of factors including the availability of compute resources and particularly the number of analysis engine instances possible in the system due to licensing or analysis implementation. Similarly the impact of linked execution between analysis nodes in the tree will reduce parallelism potential, particularly as, depending on the direction of execution, either the ancestor chain or the subtree of that node must also have completed before the dependency is fulfilled. Despite these limitations during the applications of the HierSynth framework discussed in chapter 4 (p139) generally generated sufficient parallel work tasks to utilise all computational resources for the majority of the HierSynth tree execution (as measured by the workload manager within HierSynth and contention for execution resources).

Should further parallelism be required it would be possible to consider parallelising the evaluation of scenarios under which a HierSynth tree will be executed. The case study presented in Chapter 4 (p139) frequently ran an analysis tree under 9, 10, 90, 126 or 546 scenarios. At present this particular opportunity is not exploited due to challenges in preserving data integrity and avoiding deadlock since all scenario runs are stored in the same HierSynth tree and indeed in the same tree nodes. This should be addressed in future work. Finally the overlapping of execution phases could be enabled since once a node is expanded it can be executed without waiting for the other nodes in the tree to be expanded, although this depends on the direction of execution. Similarly once a subtree is executed it can be aggregated without needing to wait for other subtrees to be executed. This could again boost performance by avoiding valleys in the number of tasks available over time as one phase of execution completes and another starts.

Table 3.2 (p123) presents a summary of parallelisation opportunities exploited and enabled by the HierSynth data model coupled with the challenges it faces. Finally we note that scheduling of concurrent tasks is currently undertaken by relying on the $\mathrm{C \#}$ 
Task Parallel library and keeping a large pool of tasks ready to be executed. Contention for resources is managed by an application sharing framework which we will now discuss.

\subsubsection{Application Sharing}

One challenge presented by the analysis applications automated by HierSynth is that they are frequently licensed or constructed in such a way that only a limited number of instances can be created. Similarly limits on computational resources will restrict the number of instances of an application that it is possible to run concurrently. For these reasons and to manage concurrent access to application instances an application sharing framework was included in HierSynth. This framework mediates access to application instances by asking all analysis nodes to request application instance from a management class. This manages access to the applications by only returning a interoperability class when application resources are available. This interoperability class interfaces with the external application by providing routines for analysis setup, processing and post-processing. The application manager must be threadsafe which presented a few challenges in implementation. The framework is extensible, supporting new types of analysis engine via the implementation of a new interoperability class. These classes are annotated with meta data indicating the maximum number of concurrent instances possible along with information on compute resources required. At present scheduling takes place according to the maximum number of instances possible. This is set according to per machine extensible configuration files within HierSynth leaving the operating system to mediate compute resources. However this should in future be incorporated into the application sharing framework. Finally this framework has another substantial benefit. Since each request for an application instance contains some indication of the analysis set up information it is possible to schedule application requests so as to reuse application instances avoiding start-up, configuration and close down costs of the analysis engines which can prove substantial. Thus the framework enables similar execution runs to occur sequentially on the same execution engine, this was a key technique for improved performance in Chapter 4 (p139). This was particularly useful as it enabled one instance of Microsoft Excel to open a particular analysis model and for this instance to be used for multiple analysis runs without constantly closing and re-opening the application. Together with mediating access to resources this was a key technique in making the HierSynth data model perform well enough to return results in under an hour in most circumstances despite having run up to several thousand analyses. 


\subsubsection{Caching}

Finally a results caching framework was added into HierSynth to avoid re-computation of analysis results computed earlier in the analysis run or in previous executions. This was primarily applied to long running analysis jobs (such as Radiance analyses). Caching occurs at the level of requests for access to external resources and applications. This is because these requests contain sufficient information to uniquely identify the objects. The actual caching algorithm is uniquely defined by the application interoperability code within HierSynth which exposes the application to execution nodes via the application sharing mechanism discussed in the previous section. Such implementations have access to several internal methods to support caching. Particularly, the framework enabled the key for the analysis to consist of strings, serialised objects, model version numbers and file/directory checksums which represent the inputs to the analysis model. A major use for this framework was for caching external configuration data such as lists of KPI's to read from specific cells in an spreadsheet model. The framework was not applied to short-running analyses such as Excel based model to avoid cache pollution as it was expected that most analysis runs would be different. This caching framework was able to approximately halve the number of application queries required to run the HierSynth tree developed in section 4.9 (p157) by caching lists of input locations to write to within an Excel model and similarly caching the list of output KPI's to read.

\subsection{Implementation}

The HierSynth framework is implemented in C\# 4.5 under the .Net framework and consists of a core of around 30,000 lines of code supported by 18,000 lines of addins enabling insight and interoperability and takes advantage of heavily modified version 10, 000 line expression language NCalc [85].

HierSynth takes advantage of .Net frameworks such as Language INtegrated Query (LINQ) [97], Task Parallel Library and the Managed Extensibility Framework (MEF) [98] which enabled construction of the HierSynth framework. The HierSynth GUI is shown in Fig. 3.17 (p126).

HierSynth uses a consistent means of presenting information to users via a tree based interface based upon Infralution's Virtual Treeview component [50] backed by an XML document together with a set of anonymous functions defining actions to be undertaken to extract a tree from the XML document, display information in each column as well as responding to edit events. Each configuration panel follows this method and so presents a coherent and simple to follow environment for users. Frequently the XML document backing a configuration panel is a serialized version of an object in the HierSynth data model which enables simple editors to be prepared from annotations to the objects type read via reflection. Similarly HierSynth uses XML based serialization as the primary 


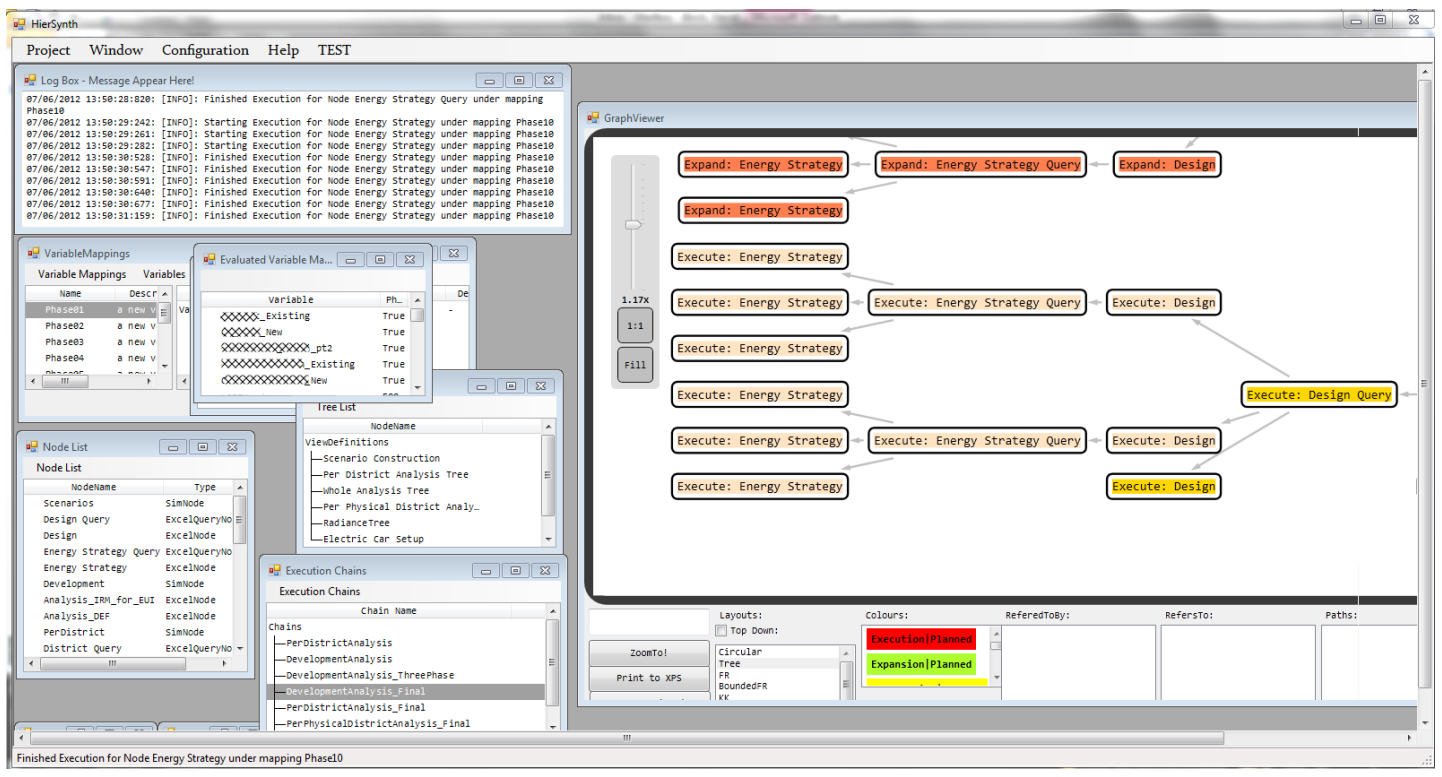

Figure 3.17: The GUI of HierSynth, showing configuration on the left and the execution of a small HierSynth tree on the right.

means of saving and loading projects and results.

HierSynth uses the Unity3d [100] visualisation engine to enable $3 d$ visualisation of results. This is done via a Unity3d custom application reading configuration data produced from HierSynth. Unity3d was chosen for its web based and cross platform support as well as easy of development.

Finally the HierSynth platform was built to be highly extensible via an addin framework.

\subsubsection{Plugins}

HierSynth uses the Managed Extensibility Framework (MEF) in C\# to enable new plugins to be loaded from dll files in a plugin directory. HierSynth includes a configuration system for each plugin allowing simple standardised configuration of each plugin. Numerous plugin points are provided and are listed below:

- New expression language functions can be incorporated into the NCALC library via extensions made to the library to support MEF.

- New analysis nodes can be added to support different analysis programs, they must implement a standard execute method as well as listing the variables they expect to use. New analysis nodes should work with new application interoperability modules.

- New applications interoperability modules enabling application sharing and concurrency management for new data sources and analyses. 
- New query nodes allowing different data sources to be queried, examples include Excel and GIS shape file readers.

- New analysis for using with an execution chain - for example new forms of sensitivity analysis can be incorporated into an execution chain as shown in Fig. 3.18 (p130)].

- New datalayers for use in building performance trees with custom aggregators as described in Section 3.8 (p108).

- Insight-giving result viewers - these are addins which enable exploration of an analysed HierSynth tree. This include a performance tree visualiser and a graph generator.

- Data sources can be read in from different sources (e.g. Excel, text file) or generated, new plugins allow more data sources.

- Data Series - means of extracting data sets from an executed HierSynth Tree.

- Graph generators - means of composing data series into meaningful graphs

- Graph viewers - means of visualising generated graphs.

One of the most successful plugins to the HierSynth framework is a sensitivity analysis module which is a plugin analysis for use in an execution chain.

\subsection{Sensitivity Analysis}

As discussed in section 2.7.2 (p68) sensitivity analysis is a key technique used in product design to explpore the design space and identify the best means of improving a design. HierSynth supports two methods of running a sensitivity analysis, depending upon the complexity of the analysis required and the experiment being analysed.

First a single HierSynth tree can be used as the experiment to analyse. In this case four scenarios are provided. One provides a boolean value for each variable in the scenario indicating whether it is a factor to be analysed by the sensitivity analysis or not. For variables not involved a default value to be used throughout the analysis is specified in a second scenario. The final two scenarios provide high and low values for each factor. The PB analysis module takes these four scenarios and uses them to produce a large number of experimental runs. Each of these runs is actually a new scenario and the HierSynth tree will then be executed for each of these scenarios. Once this is complete the PB analysis module will analyse the results and compute sensitivities storing them within the HierSynth tree as a new scenario. 
The results of a sensitivity analysis on a single output KPI is the list of factors together with a measure of the KPI's sensitivity to each. This enables identification of the most effective ways to impact that KPI by changing design parameters within the limits identified by the high and low values used in the sensitivity analysis. Similarly for analysis assumptions where the range of inputs represents uncertainty, a sensitivity analysis will identify which uncertainties the KPI is most sensitive to and how the analysis can be made more accurate by resolving them. The number of factors to which the KPI has non-negligible sensitivity is also interesting as it shows the range of options open to the design team or alternately a small number key design variables to focus upon. Examples of the insight that a sensitivity analysis can generate for a single KPI are shown in Figs. 4.28 (p188) and 4.29 (p189).

In contrast to normal practice, HierSynth computes a sensitivity analysis for all outputs of the experiment. Within the HierSynth data model this means that for each of the outputs and datalayers on all nodes of the HierSynth tree a list of factors which it is most sensitive to is identified. This presents several intriguing potential insights:

- Comparison of sensitivities across design element peers - Identifying why certain suburbs or buildings are more sensitive to changes in one analysis assumption or design parameter could identify a key design change.

- Comparison of sensitivities across design decomposition - Identifying how the sensitivity to changes in design parameters changes between a design element and the elements of it's decomposition would identify whether the scope for impacting smaller design element's performance will actually effect the biggerpicture shown by the performance of the larger design element.

- Comparison of sensitivities within a performance (or preference) tree - May identify key means of improving the design for different stakeholders, particularly if the performance tree is composed of performance trees from different disciplines.

- Comparison of sensitivities across peer performance (or preference) trees - May identify which parameters give scope for resolving stakeholder concerns across different areas of the designs (e.g. different suburbs).

- Comparison of sensitivities across performance (or preference) trees in the design decomposition - This may identify whether the same design parameters which impact smaller scale concerns have similar potential impact to address the bigger-picture concern across the whole design.

In this way we expect to be able to generate much design insight using a sensitivity analysis. As an example Fig. 5.11 (p230) identifies some of the benefits of analysing 
sensitivities within a performance tree - in this case a tree formed by the summation of different sources of operational carbon. It is possible to identify the contribution of each source of carbon via its sensitivities. For example transport emissions are highly sensitive to heavy rail transportation as is the total from all sources. This indicates the importance of the transport component of total annual carbon emissions. We are able to use this technique to identify the best 20 options for impacting carbon emissions whilst tracking their side effects upon other KPI's of interest such as electricity consumption. This is a key insight for the engineering and design teams as will be discussed in Chapter 4 (p139).

\section{Sensitivity Analysis in Execution Chains}

Since the main use of HierSynth is likely to be using execution chains (section 3.10 (p114)) we now consider how sensitivity analyses can be integrated into an execution chain. At its core an execution chain enables one analysis tree to generate scenarios for the next tree in the chain to analyse. This can be done via scenario generation, composition or as a result of external analysis results. The integration of sensitivity analyses within execution chains is achieved by splicing experiment generation and sensitivity analysis modules into the execution chain.

As we have seen in Fig. 3.1 (p118) an execution chain containing a sensitivity analysis may be as follows. The goal of such an analysis might be to investigate the sensitivity of sustainability metrics to varying the development density in several (say 14) districts in a development. This would enable insight into the best areas of the development to develop as a central business district.

0. Initial Scenario

1. $[\downarrow]$ Compose Designs with Carbon Strategies

2. Generate Sensitivity Analysis Experiments

3. [个] Analyse Design

\section{Analyse Sensitivity Analysis Experiments}

This execution chain is undertaking analysis of a set of designs under a set of energy strategies ("Good", "Better" and "Best"). A sensitivity analysis is to be performed upon a number of design parameters. The execution chain consists of two HierSynth trees 1) and 3) together with a sensitivity analysis module spliced between them. This execution chain is shown visually in Fig. 3.18 (p130).

Initially (0) a basic scenario is chosen, in this case the final (tenth) construction phase. This scenario will be used to run the execution chain. For this initial scenario 


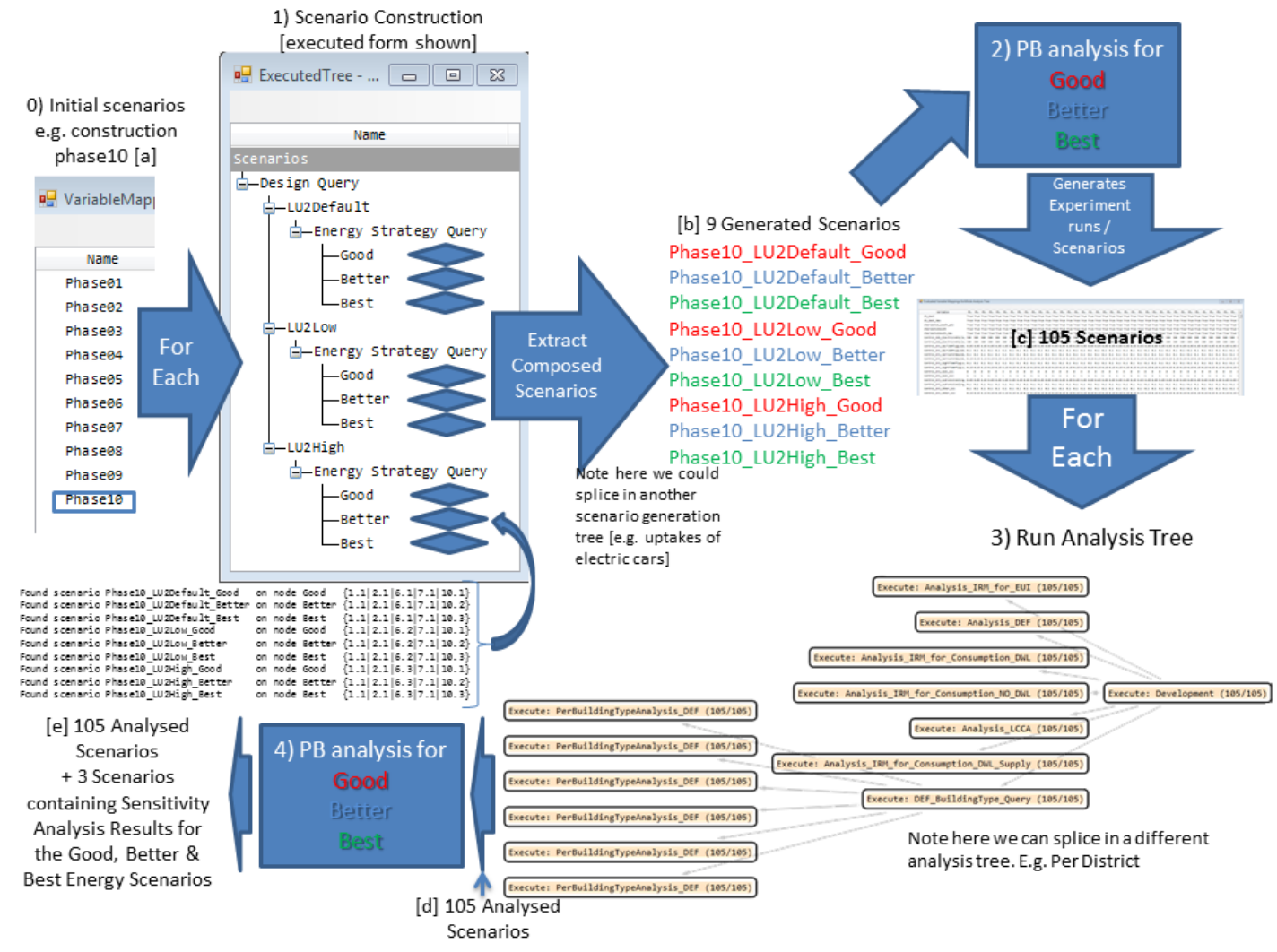

Figure 3.18: An example execution chain consisting of two execution trees, one for scenario generation and another for analysis. Into this chain is spliced an experiment generation module for a sensitivity analysis and its corresponding analysis module is used at the end to compute sensitivities. 
$[a]$ the first HierSynth tree (1) is executed to generate a number of analysis scenarios using downward execution of design and scenario queries with scenario composition as discussed in more detail in section 4.6 (p148). This tree then generates the 9 scenarios shown in $[b]$ (one extracted from each leaf node) which compose three designs ("Default", "Low" and "High") under three energy scenarios ("Good", "Better" and "Best").

At this point (2) the first part of the sensitivity analysis is performed, namely the generation of experiments. In this case sensitivity to design parameters is being explored. Hence three sensitivity analyses will be carried out - namely one for each of the three energy scenarios ("Good", "Better" and "Best"). For each of these, the set of designs "Default", "Low" and "High" will become the inputs to a sensitivity analysis. The sensitivity analysis uses these three scenarios, together with a previously configured list of factors to analyse, to generate a set of experiment runs. This occurs for each of the three energy strategies resulting in three separate sensitivity analyses occurring in a single run of the execution chain.

The first sensitivity analysis module (2) analyses sensitivity to 14 factors. This produces some 105 experiment runs $[c]$; this is comprised of the original 9 scenarios plus 32 experimental runs for each of three sensitivity analysis ("Good", "Better" and "Best"). Each sensitivity analysis contains 32 runs since because we analyse 14 factors we must use the next largest PB design matrix which has 16 rows each of which becomes an experimental run; we then use a technique ("Folding" see section 2.7.2 (p69)) to separate main factor effects from their interactions, the cost of this technique is to double the number of experimental runs.

Having generated these experiment scenarios $[c]$ the second HierSynth tree (3) is executed for each of them. This tree will analyse the generated design under the energy strategy using a number of analysis modules as described in section 4.9 (p157). Each execution of this tree (3) will generate some 1,315 output KPI's across the 12 analysis modules shown in analysis tree 3 ) together with a performance tree for each analysis module. This tree could also have analysed the design district by district using a design decomposition if required which would have enabled exploration of changes in sensitivity over the design decomposition.

The KPI's generated by the analysis tree (3) under each of the 105 analysed scenarios $[d]$ are then analysed by the sensitivity analysis module (4). For each sensitivity analysis conducted, a new scenario will be created $(e)$ which stores a set of sensitivity analysis results. For each output and datalayer on each node of the analysis HierSynth tree (3), a set of analysis results is stored in these new scenarios. In each case a list of the factors and the output's sensitivity to them is stored. Examples of these lists are shown in Figs. 4.28 (p188) and 4.29 (p189). 


\subsubsection{Possible Extensions}

We note it is possible to extend this execution chain to investigate further aspects of the design space. For example other scenario generation trees can be spliced between (1) and (2), perhaps to investigate different levels of electric car uptake. Additionally the execution chain could be run under more initial scenarios, perhaps to see how sensitivities change over time, giving insight into which design parameters may be the most important during construction of the masterplan.

Since many different sensitivity analyses can be performed using an execution chain (for example Fig. 3.18 (p130) performs three), it may be interesting to consider changes in sensitivity patterns between different designs and scenarios, particularly across the list of potential insights list above. For example how does a more dense design change the most effective design parameters for impacting percapita carbon emissions? Additionally, more subtle insight may be generated perhaps investigating how the move from 5 small suburbs to 3 larger suburbs affects the sensitivities identified not only across the different suburbs but also at the city level. This can be considered particularly by comparing aggregate metrics computed using datalayers and performance trees using data from the set of child suburbs to compute for example the average annual carbon emissions from a suburb. Factors which a KPI is no longer sensitive to may have been specific to only one suburb, or have had a particular impact upon smaller scale communities.

The HierSynth framework is able to use these same means of running sensitivity analyses with other sensitivity analysis algorithms which are supported via a plugin system. These will give more insight but at a greater computational cost. In conclusion sensitivity analysis proved a valuable capability and was used to good effect in the case study discussed in section 4.13 (p187).

\subsection{Visualisation}

Another plugin of critical importance to HierSynth is the visualisation engine. This enables automated geolocated visualisation of design and analysis results. This allows interactive exploration of results, understanding of spatial trends, comparing scenarios and serves as a highly useful discussion tool for the design and analysis teams together with the client and other stakeholders [93]. HierSynth uses a customised Unity3d [100] viewer and can generate $3 \mathrm{~d}$ geometry from GIS data. HierSynth uses geolocated performance trees (e.g. Fig. 4.16 (p170)) to visualise analysis results and design properties together enabling comprehension of the design differences between design components allowing the reasons for trends and potential changes to be explored. The visualiser is able to render many sets of performance trees and designs each highlighting a different aspect of the designs performance, allowing different discussions to occur as required to 
improve the design. The visualiser itself is extensible and is able to cope with displaying multiple discipline's analysis results in a similar manner to [65], particularly enabling the visualisation of lighting maps computed with Radiance [103]. This extension point could be used to support other physical analyses such as wind flow. Visualisation is expected to be an important technique for speeding up the analysis cycle by providing rapid visual comprehension of results as well as involving the client via easy comprehension of design performance allowing greater input into the design process and hopefully improving its performance. This visualisation environment is evaluated in section 4.15 (p199).

\subsection{Workflow}

Before closing this chapter it may be useful to give an overview of the expected workflow using HierSynth. In relation to the urban masterplanning design and analysis cycles shown in Fig. 3.1 (p86) HierSynth sits between the two cycles. Specifically HierSynth should be able to read the design teams files, run preconfigured analyses and return analysis results to the engineering and design teams. HierSynth should be able to improve the speed of the analysis cycle to keep up with the design cycle allowing more iterations.

Currently HierSynth will require an expert operator to run. This will require an extra team member working with all practitioners on the analysis team to integrate their models and run analyses for them, returning results of interest to them. Similarly interaction with the design team will be needed to receive design files for each design cycle and hopefully to return insight in the form of automatic visualisations and graphics produced via the framework. To give an idea of the workload involved in running HierSynth, during the case study described in Chapter 4 (p139) the average time to incorporate a new model into the HierSynth framework was around 2-3 days of effort. This is primarily in terms of understanding the inputs and outputs of the model. The setup time within HierSynth was less than an hour per model. This however will rise when custom scripting is required (e.g. for a CFD model). The plugable nature of the platform meant that many configuration details could be reused many times and most design space investigations were set up in an hour at most. The primary time consuming task was the integration of models together to form an ensemble due to the complexity of each model, the need to harmonise assumptions and to integrate them together. This task took several weeks, which reflects experiences of other researchers [36].

A common expectation from the use of greater computation in the analysis cycle is that it will make the analysis engineers redundant. However practical experience as discussed in Chapter 4 (p139) shows that whilst automating simple tasks there is still 
a large requirement for expert analysis of results to produce insight of interest to the client. Indeed if anything the greater and more detailed exploration of designs and the design space requires more expert input, not less.

\subsection{HierSynth in Context}

Finally this section aims to place HierSynth in the context of the literature and projects discussed in Chapter 2 (p37). We now discuss the relationship and potential partnerships of the HierSynth framework with the following communities:

\subsubsection{Geographic Information Systems}

These are a widely used set of platforms for creating, storing and processing detailed geographical data sets. They are not normally used by the design team but are frequently used in analysis - for example ensuring a development always has a bus stop within five minutes walk. The HierSynth platform could work with this community in three ways:

- Firstly reading design and contextual information from GIS databases.

- Secondly using GIS systems and analyses as part of the automated toolkit used to assess designs.

- Finally HierSynth has the capacity to export results to GIS databases.

Indeed it may be that GIS practitioners have the best mix of skills needed to run a system such as HierSynth, particularly given their organisational position as collectors and maintainers of datasets derived from designers and many disciplines.

\subsubsection{Computational Design Optimisation (CDO)}

Computational Design Optimisation (CDO) is a wide field of research which is slowly being adopted into architectural practice [89]. The HierSynth platform is to designed to support the view that the critical mission of computation is not to automate design improvement but rather to provide insight to the practitioners who improve design. For this reason the HierSynth platform does not currently contain an optimisation loop. However one could be added relatively easily. Particularly this would require an optimisation engine and integration with form generation tools such as parametric CAD tools [84] [41]. This would enable the optimisation of specific challenges (e.g. structural integrity vs weight) which would support practitioners in addressing the more complex challenges such as sustainability. 


\subsubsection{Interoperability Standards}

As discussed in section 2.8 (p71) there are many integration systems and models covering many areas of the AEC industry. HierSynth has taken the following approach to integration and cooperation with these projects:

- Enable Integration - HierSynth supports reading data from several commonly used industry data sources and formats such as GIS shapefiles and Wavefront .obj 3d models. Further integration should be undertaken to add query nodes within HierSynth that query standard formats such as CityGML, gbXML and IFCs.

- Exploit Hierarchy - Many of the integration formats are based in XML and exploit hierarchical decompositions of the city and building designs they model. Since HierSynth enables analysis and insight at all levels of design through design decomposition there is a natural bridge to extract the hierarchies within existing formats and represent them in HierSynth. Whilst this can already be done using the framework, further interoperability plugins and query nodes within the HierSynth framework could support this more rapidly. For example a single query node reading an IFC model might create a HierSynth tree decomposition of a building's steel frame broken down by floor, section and member.

- Create Views - Since these file formats contain a large amount of complex data from many disciplines there has been recent work in trying to create query systems to create consistent "views" of the model which contain only the information specific to a single practitioner. This is also an approach HierSynth already follows. In the future HierSynth might take advantage of this research by incorporating such query languages [46].

- Allow Export - HierSynth already support export of data to several formats; further work should enable export of analysis results back into further industry formats. This would make the HierSynth engine an integral part of the design and analysis workflow.

Hence we see that the HierSynth data model is complementary to many of the plethora of industry formats through its plugin system and use of query nodes to form a "view" of the design through a design decomposition embedded within a HierSynth tree.

\subsubsection{Design / Analysis Suites}

There are many analysis models in this industry, HierSynth aims to allow integration and where possible automation of standard configurations of these analyses. As discussed in Chapter 2 (p37), also developing are more tightly integrated analysis suites 
such as [10] [49] which contain several discipline's analysis tools. These tools normally include a direct design tool as their basis.

These tools are a form of direct competition for HierSynth and indeed for all architectural analysis practitioners. In taking the analysis tools, simplifying them and providing them as tools for the designer they aid the design process directly. However there is an argument that they provide only simplistic analyses and without the insight and assessment possible through engaging with expert practitioners. HierSynth aims to take advantage of this expertise by aiding analysis practitioners to set up analyses within HierSynth and then review the results either with or before the client sees them. This, of course, loses the immediacy of feedback, but it remains a substantial improvement on current design / analysis cycle speeds [35]. It also gains the benefit of much deeper analysis and insight that is possible through such design / analysis suites.

\subsubsection{Building Scale}

HierSynth is currently targeted at the urban masterplanning scale where challenges of data management and scalability are more acute than at the building scale where interoperability and design complexity are more acute. However there is nothing within the HierSynth data model which is specific to urban masterplanning and the framework could be targeted to building design instead. This would involve a new set of plugins targeting building design file formats and common building analyses.

\subsection{Conclusions}

This chapter has presented the design of the HierSynth framework. The framework has been developed to address the specific needs of the urban masterplanning community. The novel compositional data model gives the capability to improve the speed of the analysis cycle. This will enable more analysis iterations and more quantitative design feedback. The capabilities of the framework are aimed to generate design insight for practitioners and to enable design space exploration to answer practitioner questions.

Specifically the HierSynth data model re-conceptualises tree based data models and applies them to the context of architectural workflows. The HierSynth data model provides a tree based design decomposition which puts the design back at the centre of workflow automation through its use of query nodes to read data from architects and analysis models to run engineering analyses at various scales within the design decomposition. This coupled with workflow automation and larger design space exploration techniques will enable substantial speed up and improved insight over previously non-automated workflow. A case study exploring these benefits and the efficacy of the HierSynth data model is discussed in chapter 4 (p139).

The major distinguishing features of the HierSynth platform are: 
- A focus on providing a unifying computational platform to both the design and analysis cycle.

- Scenario Composition system - using the standard analysis model to create new scenarios for analysis.

- Performance trees per design element - giving detailed insight.

- Design elements are first class citizens with query and analysis modules. Design elements (buildings, streets, districts) are critical to the design and analysis teams, hence HierSynth ensure they have equal standing with design queries and analysis modules in the data model.

- Composition of sensitivity analysis into an execution chain - enabling sensitivity analysis results across all design elements and performance trees. Multiple sensitivity analyses can be performed simultaneously across multiple designs / scenarios. This enables more insight into varying sensitivities across the design space.

Having created and explored in detail the HierSynth framework we now explore whether or not the framework and the techniques it uses will be effective on a live urban masterplanning project. We will seek to test each of the techniques and features of the data model and explore their effectiveness in meeting the challenges identified in Chapter 2 (p37). 



\section{Case Study - CommunityB}

This chapter introduces a case study application of the HierSynth framework to a live project in collaboration with Arup North America. It will discuss the challenges which the project team encountered and the insight which using the HierSynth platform gave. This will be followed by a critical analysis of how and where the HierSynth framework was applied and adapted to address these challenges. Finally a number of design insight questions were investigated via the HierSynth framework, these will be discussed in the light of the strengths and weaknesses of the HierSynth framework. Due to the need for commercial confidentiality the results presented here have been anonymised.

The work presented in this chapter would not have been possible without the expertise and support of the analysis team from Arup North America.

The work described in this chapter would not have been possible without the expertise and support of the Arup design team involved in this project. Their help and support is gratefully acknowledged.

\subsection{Introduction}

This chapter explores a collaboration with Arup North America to apply the HierSynth platform to analyse the CommunityB masterplan. The CommunityB project is a mixed office / residential development and we were working an early stage of the masterplanning process where the size and composition of the masterplan were still being actively explored. The project spanned some fourteen "districts" with a mix of new build, rebuild and retrofit being discussed over ten construction phases. The external urban masterplan design team was exploring three different designs of varying density and prepared several landuse schedules detailing the density and Gross Floor Area (GFA) for each of six types of land use (housing, existing office, new office, new laboratories, fitness centres (gyms \& swimming pools) and central services (building services)). These land use schedules along with detailed information about the masterplan area and the expected standards of construction provided the required information 


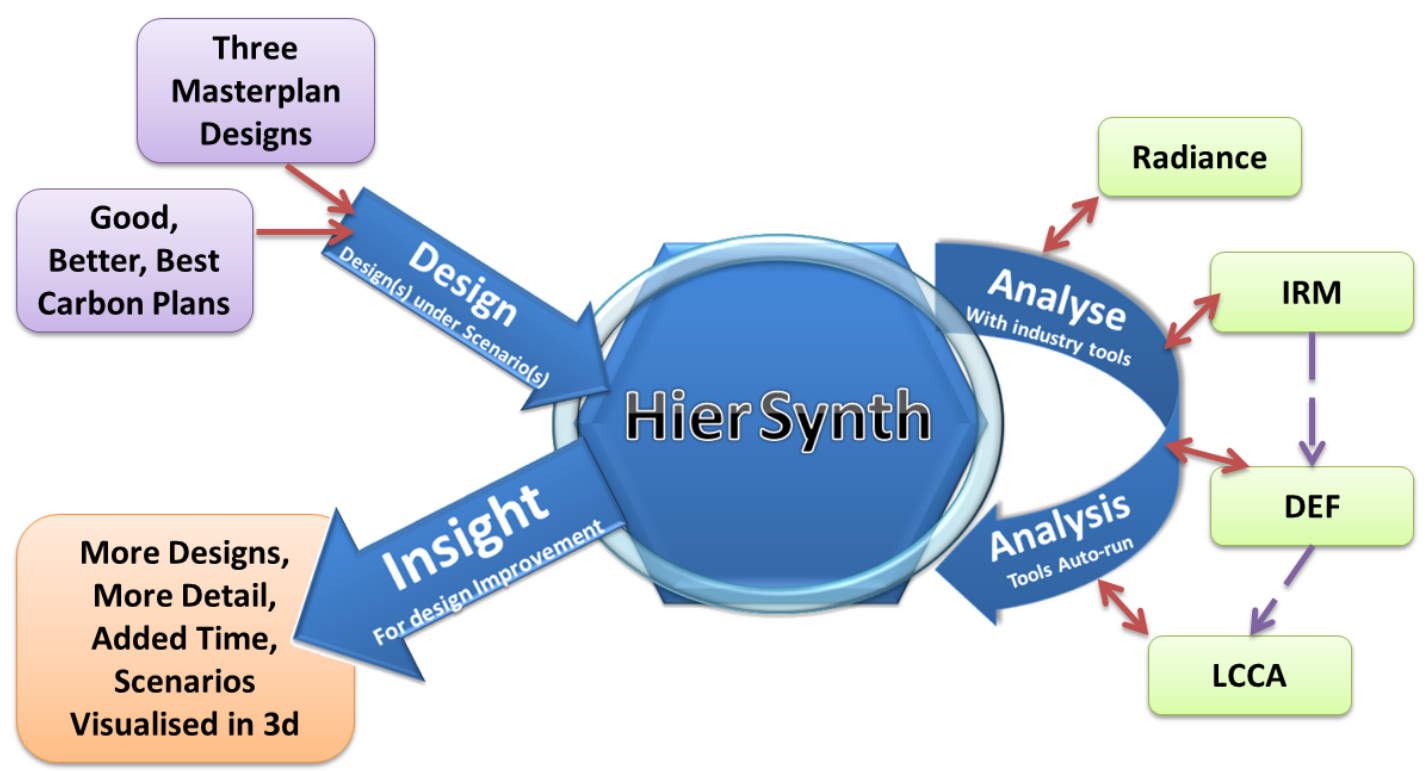

Figure 4.1: Conceptual overview of the application of HierSynth to a live project with Arup North America. Compare with HierSynth overview in Fig. 3.2 (p87). Dashed arrows indicate how HierSynth was used to integrate separate models into a large model adding capacities not available previously.

for the Arup team to undertake their scope of providing sustainability assessment and advice on carbon emissions and energy use reduction strategies particularly focusing on district energy systems. Working at this early stage of the masterplanning process meant that detailed design of the geometry of the masterplan was limited however it gave considerable scope for design space exploration in terms of the landuse schedule composition and density together with the set of energy strategies to be employed to reduce carbon emissions.

The goal of the Arup team was to assess various masterplan options for Key Performance Indicators (KPI's) such as water, electricity and gas consumption, operational carbon emissions per capita and per $M^{2}$ as well as transportation and waste KPI's.

Fig. 4.1 (p140) graphically demonstrates the use of HierSynth on the project, firstly the designs supplied by the external architect along with the energy scenario carbon plans prepared by the team were read by HierSynth. These scenarios were often composed with different construction phases or investigations into the effectiveness of certain energy strategies. HierSynth was then used to automate and integrate the team's models as will be discussed in the next section.

To achieve this the team used a variety of in-house models to assess the masterplan. Firstly Arup's Integrated Resource Management (IRM) model was used for integrated sustainability assessment across many disciplines from landuse, energy demand and supply to waste and transport. This model enabled the exploration of sixty possible 
energy strategies such as photovoltaics, solar thermal and water efficient appliances which help to reduce the environmental impact of the development. Secondly Arup's District Energy Feasibility (DEF) model was used to explore fourteen options for adding a district energy system to the masterplan with various district heating and cooling systems powered by various distributed or centralised power technology. Finally a costing model was used to explore the financial impact of these district energy systems. These three models formed the opportunity for applying HierSynth to a live project as part of the team.

\subsection{Research Methodology}

The case study described in this chapter is primarily an evaluation of the HierSynth framework as described in Chapter 3 (p85). It seeks to explore the efficacy of the HierSynth data model and its applicability to practical use in a real world project.

Ultimately the success of such an application is in the degree to which the data model was adaptable to practical challenges, enabled quantitative improvement in the speed of the design-analysis cycle and critically the degree to which it generated new insight into the designs under consideration and their surrounding design space.

At various points other research methodologies were employed to focus on particular challenges presented during the collaboration. Most notably Constructive Research was used to further develop the HierSynth framework in response to new challenges it was previously unable to meet. Most notable from this was the introduction of the execution chain concept to enable design space exploration studies.

The research methodology employed particularly during the design space exploration studies was often a form of collaborative Action Research initiated by the Arup team to explore challenges facing the design team. In this role the HierSynth platform was the key enabling technology and was used by the author to support the analysis team's investigations.

These research methodologies were employed to answer research questions from both a research and a practical project perspective which are now discussed.

\subsection{Case Study Research Questions}

This collaboration with Arup lasted around nine months was equally split between working to answer these research questions:

1. To identify whether the HierSynth framework could be applied to current practice and workflow with a view to addressing the mismatch between the design and analysis cycles discussed in Chapter 2 (p37). 
2. To explore what improved analysis capabilities are enabled through the use of a computational framework.

3. To understand the requirements, opportunities and challenges of applying a computational framework to generate insight for the project team by answering "what if" and design space exploration questions. Examples include:

a) "What is the impact of electrical vehicle uptake on the performance of the masterplan area?" see section 4.12.1 (p172)

b) "What is the optimal (or critical) density for the district energy system?" see section 4.12 .2 (p175)

c) "What is the optimal mix of residential and commercial development for heat recovery and reduced vehicle miles travelled?" see section $4.12 .3(\mathrm{p} 177)$

d) "What are the development's carbon emissions most sensitive to? Which of these sensitivities can we positively impact?" see section 4.13.1 (p187)

e) "How significant are the various (energy) strategies in reducing the developments carbon emissions and water intensity?" see section 4.13.2 (p190)

f) "Is it better to vary density (i.e. build dense in a few areas and less dense in others), or build equal density everywhere?" This question was not tackled due to time constraints.

4. To identify the efficacy of the techniques and data models included in HierSynth as applied to a live urban masterplanning project. Specifically:

- Whether simple model automation can reduce the gap between the analysis and design cycles.

- To explore the potential of scenario composition to generate design insight.

- To consider the effectiveness of model integration and the difficulty involved.

- To identify whether the HierSynth execution chain model and the HierSynth trees it contains can be effectively mapped onto the design and analysis challenges within the project. Particularly the applicability and utility of the design decomposition it enables.

- To show the potential of $3 \mathrm{~d}$ visualisation and integrated analysis within the urban masterplanning teams.

- To identify how effectively performance trees can be used to visualise performance across a design and its decomposition by showing multiple KPI's from many disciplines. 
- To explore the effectiveness of sensitivity analyses upon the models used within the project.

Evidence for the answering of these questions took several forms. In part through the success or otherwise of particular techniques employed and the lessons and experiences encountered and recorded by the author during the collaboration. More formal qualitative feedback was given and recorded during team video conferences with Arup practitioners during discussions of the results computed using the HierSynth engine. These insights were also recorded informally through phone calls and e-mails during the collaboration. Finally during the writeup process of Arup reports and this thesis additional qualitative feedback was received and is embedded in this chapter. The quantitative evaluation presented in this chapter covering the performance of the HierSynth framework and the number of analysis iterations undertaken was recorded carefully during the collaboration by the author, or were measured at a later date by repeated previously set-up analysis. Many of the insights presented within this chapter are the result of the experience and expertise of the Arup practitioners involved and their support is gratefully acknowledged.

To give a flavour of this chapter, as a result of the collaboration we were able to automate each of the models, speeding up the previous analysis cycles dramatically. We then integrated these three models into a larger more insightful model providing more accurate analysis data more frequently than previously possible. This larger model was then used to analyse 3 designs consisting of 14 districts under 3 energy scenarios over 10 construction phases producing substantially more detailed and insightful data than was previously available. The final phase of our collaboration saw the investigation of a number of insight questions which were case studies in design space exploration enabled by using HierSynth and its scenario composition capacities. Examples include "What is the impact of electric vehicles on the masterplan?" or "What is the optimal mix of residential and commercial development in the masterplan for a district energy system?", the insight generated by using HierSynth features to answer these questions will be discussed later in this chapter.

It is important to understand how the data HierSynth generated during this collaboration was used. The analysis cycle revolved around receiving new design data, scenarios or model versions. Running these models using HierSynth to produce data which was then passed to the analysis team for use in the following ways to affect the design:

1. Verification of the design and its analysis - is the design and analysis modelling activity accurate?

- Identification of design, data and model problems. 
- Examples of problems identified and fixed can be found in Figure 4.7 (p156) and section 4.9.2 (p161).

2. Validation of design assumptions and performance - is the design processes heading in the right direction?

- Checking if the design and energy strategies perform as predicted.

- Most of the results in the first half of this chapter aim at this, particularly figures 4.4 (p152), 4.6 (p155) and 4.11 (p163).

3. Guidance changeof the design process - how can insight generated guide the design process to a better solution?

- Opportunities, investigations and insight for improving design performance.

- The second half of this chapter details a number of investigations aimed at providing guidance to the design team, see particularly sections 4.12 (p171), 4.13.1 (p187) and 4.13.2 (p190).

In all of these cases the data produced must be understood to be modeling a future reality and so will not be fully accurate. Hence whilst validation and guidance are possible, the results must be taken with a grain of salt and used carefully. Frequently insight generated will lead to further more in-depth investigation to verify it, producing more guidance for the team.

Finally this chapter will discuss the insight generated by using HierSynth along with a discussion of the challenges it faced and the lessons learned.

\subsection{Models}

The masterplan analysis team used several modelling tools to complete their sustainability and energy strategy assessment. Three of these are in-house tools developed and applied over many projects and implemented in spreadsheet form by the engineers who maintain and use each model. Additionally an industry standard lighting analysis ray-tracer called Radiance was used by virtue of its previous integration into HierSynth. Each of these models was integrated into the HierSynth framework and several were integrated using the framework to form a larger model with significant new capabilities. We will now discuss each model in turn and describe the process of automating it in HierSynth.

\subsubsection{Radiance}

Radiance [103] is an open source lighting simulator used industry wide for analysing lighting conditions and designing the lighting systems of buildings. On the masterplan 
scale it is used to understand questions such as whether a street or courtyard gains enough light, whether a building overshadows another for too many hours per year or where the best places are to put solar panels. The simulator takes geometry, definitions of the materials for each model surface together with a detailed description of each light source and computes a photorealistic rendering. Several standard forms of analysis were automated and integrated into HierSynth. Fig 4.14 (p167) shows a standard radiance analysis [63].

\subsubsection{Integrated Resource Management}

Arup's Integrated Resource Management (IRM) model [12] [75] is a masterplan sustainability assessment tool. It consists of sustainability models from 8 different disciplines which are tightly integrated to mirror the interactions of resource supply and consumption we see in real life. Disciplines included are land use, energy demand, energy supply, water, waste, passenger transport, logistics and socio-economics. The IRM tool is implemented as a large complex spreadsheet consisting of over 50, 000 cells, the percapita carbon calculation alone uses approximately 1, 200 different numeric inputs across many disciplines as shown in Fig. 4.2 (p147). These inputs are of two types, firstly there are inputs related specifically to the design of the masterplan, such as the number of housing units in each district, these constitute around $37 \%$ of the inputs. Secondly there are analysis assumptions; these are numbers such the carbon emissions from an average car travelling $1 \mathrm{~km}$ or the amount of water used per $\mathrm{m}^{2}$ of office space. Some of these assumptions can be affected by the masterplanning process as results of design decisions rather than the design itself, many of these design decisions such as using low-flush toilets are encoded in the IRM model as Energy Strategies which can be turned on or off in the model and set to have varying impacts which are reflected by close integration in each discipline model. These discipline models together compute numerous KPIs across the different disciplines, for example Energy Use Intensities (EUI's) in watts $/ \mathrm{m}^{2}$ of the different building types in each masterplan.

\subsubsection{District Energy Feasibility}

Arup's District Energy Feasibility (DEF) model is used to assess the options for adding a district energy system to a development. Basing calculations upon the masterplan's land use program and taking into account localised climate patterns, energy use intensities and usage patterns of each land use type, fourteen combinations of district heating and cooling technologies are analysed and compared. Metrics for annual consumption and peak loads are computed for electricity and gas and estimates are made for the carbon emissions of for each option. Figures are computed on various time scales from hourly to annually. 


\subsubsection{Life-cycle Costing}

Arup's Life-Cycle Costing Analysis (LCCA) model for district energy systems was developed to estimate the financial cost of constructing a district energy system in a development. The model uses details of the mastherplan's land use program together with details of the proposed district energy system such as its peak load and annual consumption of electricity, gas and water to put a financial cost to a the proposed district energy system. Calculations are based on a costed pilot study and scaled up under several scenarios to obtain a range of estimates for costing.

As the reader may have spotted there is a natural flow of data between these models and they are often used in conjunction on a project, however they remain separate and non-interrelated models. One of the contributions of the HierSynth platform was to enable automated integration of the models rather than it being a manual and time consuming process. This will be discussed in detail once we have described the process of automating a model.

\subsection{Model Automation}

Automating three disparate complex engineering models was not a straightforward task, however it was achieved in collaboration with each model's user/maintainer with both parties learning much about the model. In many cases the quality of the model was also improved through the process. Since these models were implemented as Microsoft Excel spreadsheets technically the automation was as simple as identifying the inputs to enter, any processing macros to run and finally a list of KPIs to read out from the spreadsheet. However the models often required modification to enable automation and the process of automation exposed the model to a wider set of inputs and use case scenarios than normal which frequently identified bugs in the model, some of which resulted from these modifications. Specifically the automation process was as follows.

Firstly we took the model and identified all of the inputs that related to the design itself, such as the land use program (which was required in different format for each model). For the smaller models this was done in conversation with the model user with consideration to a particular use case for the model. Larger models presented a more substantial challenge. For example the IRM model contains over 50,000 calculation steps and uses over 1,200 inputs, in this case we used the "Extraction and Analysis Methodology" discussed in Chapter 5 (p209) to automatically extract the calculation graph from Excel for a particular set of KPIs. This lead to the identification of 1,224 inputs affecting the annual percapita carbon emission KPI as shown in Fig 4.2 (p147). These were then categorised and the inputs relevant to landuse, building schedule and energy strategies where automated. To do this, contextual information was encoded for each input such as its units, some of which had to be inferred from the model as they 


\section{Inputs to IRM Percapita Carbon calculation}

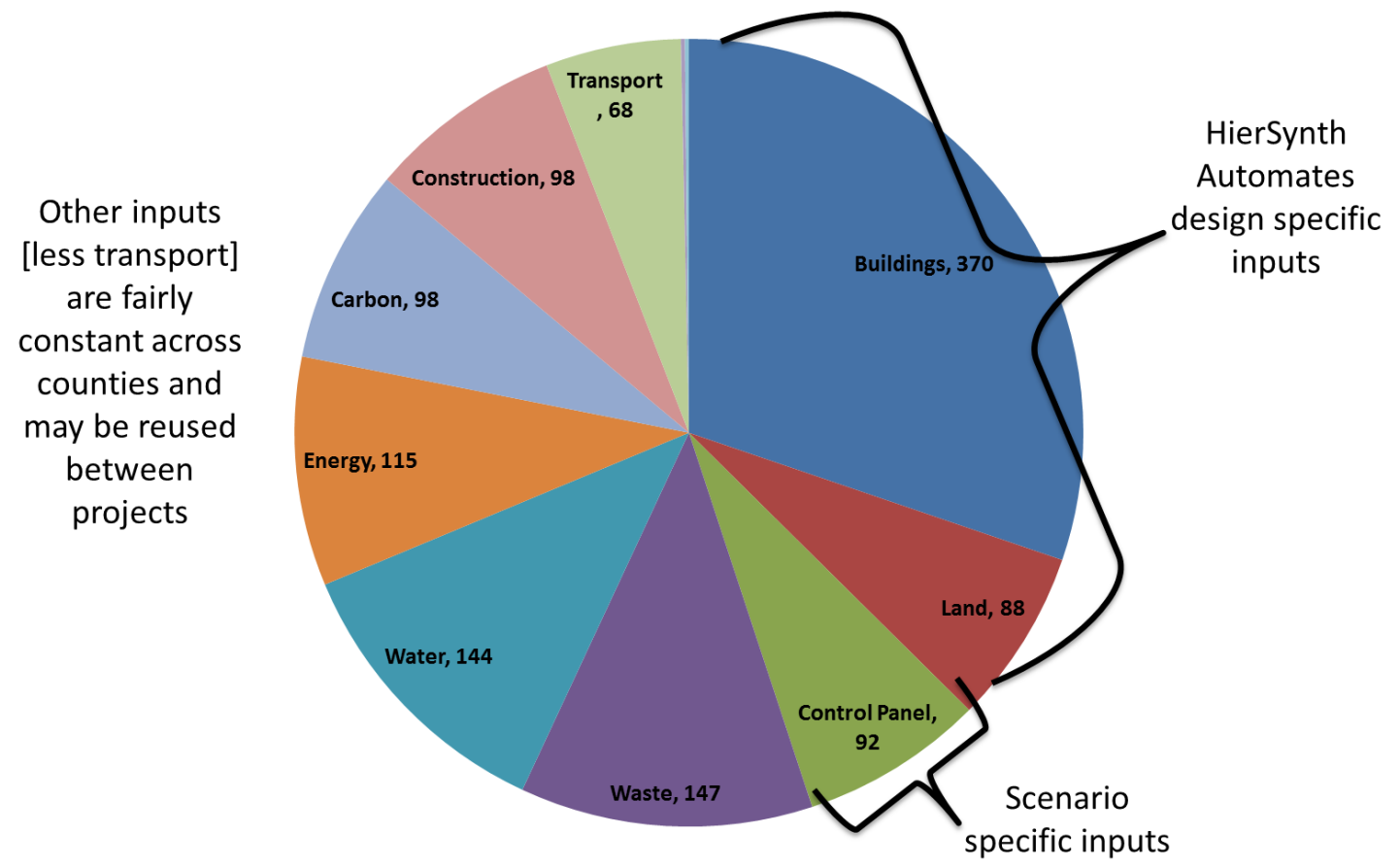

Figure 4.2: Inputs to Arup's Integrated Resource Management model's percapita carbon calculation. Extracted and classified using the tools developed in Chapter 5 (p209). Note the inputs automated by HierSynth both for analysing different designs and for analysing different energy scenarios.

were not explicit. A set of default inputs was recorded reflecting the current state of the model which had been localised to the CommunityB project, this formed the basis of the analysis scenarios we were later to explore.

Secondly we identified a large number of KPI outputs from the model, for the IRM model over 200 were used. Having formalised these input and outputs we could use this information as an operational contract of how the model operated. We used an Excel node within HierSynth to automate the design specific inputs, to run the model (and any required macros) and extract the outputs. Depending on model complexity we were able to run one model every 0.2 - 1 seconds using a quad core Intel i7 processor.

Whilst mostly automation was straightforward in some cases automated models produced unexpected results. Mostly this was as a result of assumptions and restrictions placed upon the inputs which were not explicit in the model documentation, such as cells which didn't accept zero values or tables which must be cumulative rather than annual. These generally led to minor modifications to the model. Division by zero errors were particularly frequent as they propagate through the calculation graphs and are rarely tested for by the model maintainers. As an example it is unusual to run an IRM model without any housing and doing so would result in a division by zero 
error which would propagate through to many of the KPIs. Modifying a few formulas normally provided a simple fix to these problems, however as shown in Fig 5.9 (p225) some formulas in the IRM model were over 500 characters long and proved difficult to correct. More difficult to discover were bugs surrounding the use of the model resulting in part from automation efforts, for example entering assumptions in the wrong units, which were diagnosed and fixed in collaboration with the model maintainer.

The operational contract of formalised inputs and outputs proved unexpectedly useful over the course of the project when updated models were received it was possible to identify missing or broken outputs which had not been noticed during the model modification. This can be thought of as analogous to an automated suite of unit tests, whereby over 200 output KPIs must produce results not in an error state under what was eventually thousands of model runs under numerous different combinations of scenarios. Of course not being in error state is not the same thing as being correct and as we shall see the automation of a model was often the first step in exercising ranges of inputs and parts of the model not normally used, hence identifying several bugs which were later fixed. Indeed all of these challenges to automation resulted in useful insight into how the model worked and the process of fixing these bugs has increased validity of the models.

Having automated the standard version of a model, with between 50 and 1,500 inputs and up to 200 outputs, we looked at analysing different scenarios. This involved identifying the inputs or model assumptions that would change with the scenario under discussion (for example energy efficiency of appliances over time). For each scenario the set of model assumptions/inputs to be changed for each family of scenarios was noted and was set up in HierSynth to be changed based on the values stored in the scenario. The first set of scenarios we explored was to add analysis over time to what was previously a "point in time" analysis. This will be discussed in section 4.8 (p153).

This automation process produced many benefits for the team, such as being able to produce analysis results and graphics far faster and with more accuracy, particularly when these results were generated for each scenario (e.g. construction phase or energy strategy), however greater value was found in using HierSynth to integrate these three models together, this will be discussed in section 4.9 (p157).

\subsection{Scenario Generation}

Having automated each model we were then able to consider using HierSynth to analyse different designs and different scenarios and to do so in more detail than previously possible.

The first objective was to analyse the three different landuse designs each of which had a different density and population level (low, medium and high). We were also 
interested in the different energy scenarios embedded within the IRM model; namely: a business scenario known as "Good", an affordable set of carbon reduction strategies known as "Better" and an expensive aspirational set "Best". As shown in Fig 4.2 (p147) each design would previously require the entry of several hundred inputs from the landuse schedule into the IRM model, similarly each energy strategy required a number of configuration details to be entered, for example regarding the uptake of water efficient fixtures and fittings. Notice that these two families of scenarios (designs and energy scenarios) affect separate sets of inputs, this enables them to be composed; for example analysing a low density design under a "Good" set of energy strategies.

This composition of scenarios is enabled by using a HierSynth scenario generation tree (see section 3.11 (p120)). As shown in the upper part of Fig. 4.3 (p150). The tree consists of a two nested Query-Template pairs. Firstly HierSynth queries a list of designs to analyse, for each of these a new design node is instanced. This design node then reads in the inputs that represent the land use schedule of that design which will eventually by entered into the analysis models to be run. For each of these design templates an energy strategy query is performed (this gives the possibility that some energy strategies may not be applicable to certain designs). For each energy strategy found, a template node is instanced which reads in the details of that strategy. This process gives rise to the lower tree in Fig. 4.3 (p150). In this case when executed the queries in the tree will query rows from Excel spreadsheets and the instance nodes will read data from other Excel spreadsheets. Spreadsheets are used since they are one of the primary tools architects and engineers use for sharing data. However in principle the queries could have searched for files in a directory and the designs have read directly from annotated CAD models, GIS or BIM servers.

From this generated tree we can extract the composed scenarios. Since the tree has been executed using downward execution as described in Section 3.11 (p120) we can extract 9 composed scenarios from the leaf nodes of this tree. Specifically each node of the tree modifies the variable map ("scenario") of its parent. This means that the initial scenario is patched first to contain design data and then subsequently to contain information on the energy strategy. This patching is done separately for every path through the tree from the root node to the leaves. Patching occurs at every node though in this case only the design instance and scenario instance nodes actually patch the scenario. This results in the nine leaf nodes shown in the lower tree of Fig 4.3 (p150) producing nine scenarios produced from the cartesian product of the set of designs and of scenarios.

These nine scenarios formed the basis of the analysis work carried out by the Arup team. Using this HierSynth tree for scenario generation we were able to automate a process which would have previously taken many hours of work, the results of which are discussed in the next section. 


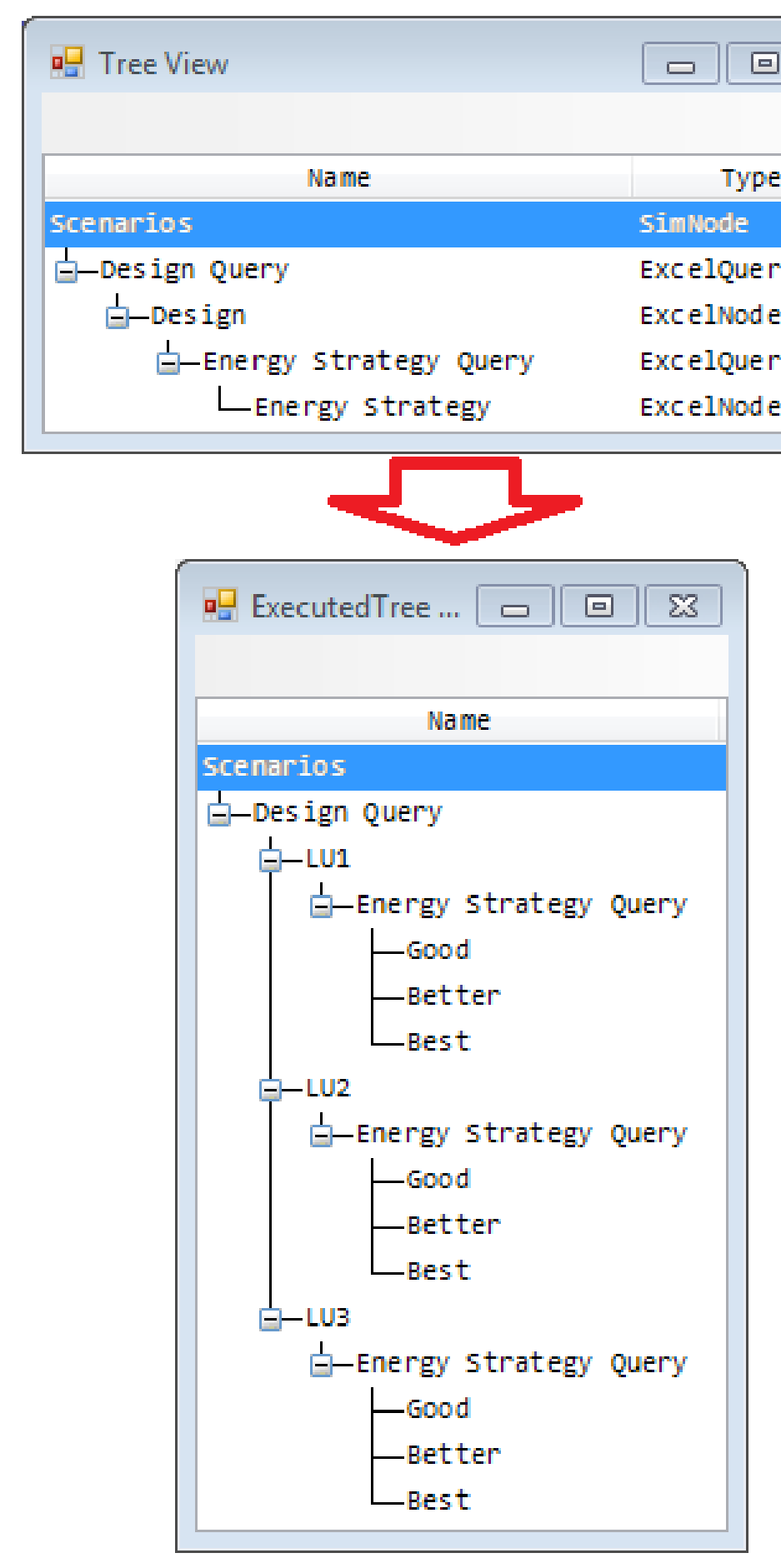

Figure 4.3: This HierSynth tree is used to compose the scenarios under which analyses will be performed. The upper image shows the tree before execution, after execution we see that each query has executed and produced 3 designs and 3 energy strategies. In this case each leaf nodes of the tree becomes a new scenario. Each level of the tree has modified (patched) the scenario with data specific to that design or energy strategy. 


\subsection{Automated Results}

As stated earlier the first research question this case study aimed to answer was to identify whether the HierSynth framework could be applied to current practice and workflow with a view to addressing the mismatch between the design and analysis cycles. Specifically for this project it meant using the now automated IRM model to analyse the three designs under the three energy scenarios under consideration. Previously this would have been a manual process of entering different landuse plans into an IRM model then extracting the relevant KPIs under the "Good", "Better" and "Best" energy scenarios. This process would have taken several hours of manual effort. However using the IRM model integrated into HierSynth and the scenario generation capability just discussed we chained together this scenario generation and a simple HierSynth tree running the IRM model which enabled the analysis of the nine scenarios in under a minute. Including pre and post processing HierSynth enabled the completion of an analysis cycle in around half an hour, substantially improving upon previous workflow. Indeed over the course of the collaboration we were able to run this analysis cycle some 14 times with improved models and different designs, a marked improvement upon the normal $2-3$ analysis cycles identified in [35].

Fig 4.4 (p152) shows the kind of analysis results this execution chain was able to generate. It shows a break down of the sources of percapita carbon emissions in the development extracted from the IRM model under three designs of increasing density and three energy scenarios. This immediately conveys and enables quantification of a number of expected trends in carbon emissions. For example the trend for less carbon percapita as both density increases and as the level of investment in energy strategies increases is not surprising but there is value in quantifying this, not least in discussion with clients and other stakeholders such as planning authorities. Similarly transport, electricity and thermal sources of carbon were expected to dominate, however we can see the disappearance of thermal as due to the introduction of a district energy system under the "Better" and "Best" energy strategies. Finally we see that transport carbon becomes a larger fraction of the total as increasing density and more energy efficiency strategies are applied to reduce other sources of carbon. This presents an increasingly enticing opportunity for tackling polluting petrol cars perhaps via the introduction of electric vehicles as is explored in section 4.12 .1 (p172).

Similar figures were also produced for total annual carbon emissions and emissions per $M^{2}$. Using the other KPIs extracted from the IRM model several dozen other comparative graphs were produced showing variation in water, electricity or gas supply and demand, waste production, transportation by mode and so forth. Each of these graphs would have taken several hours to produce manually. This increased the level of detail and will provide a more quantitative basis to the design cycle of the urban 


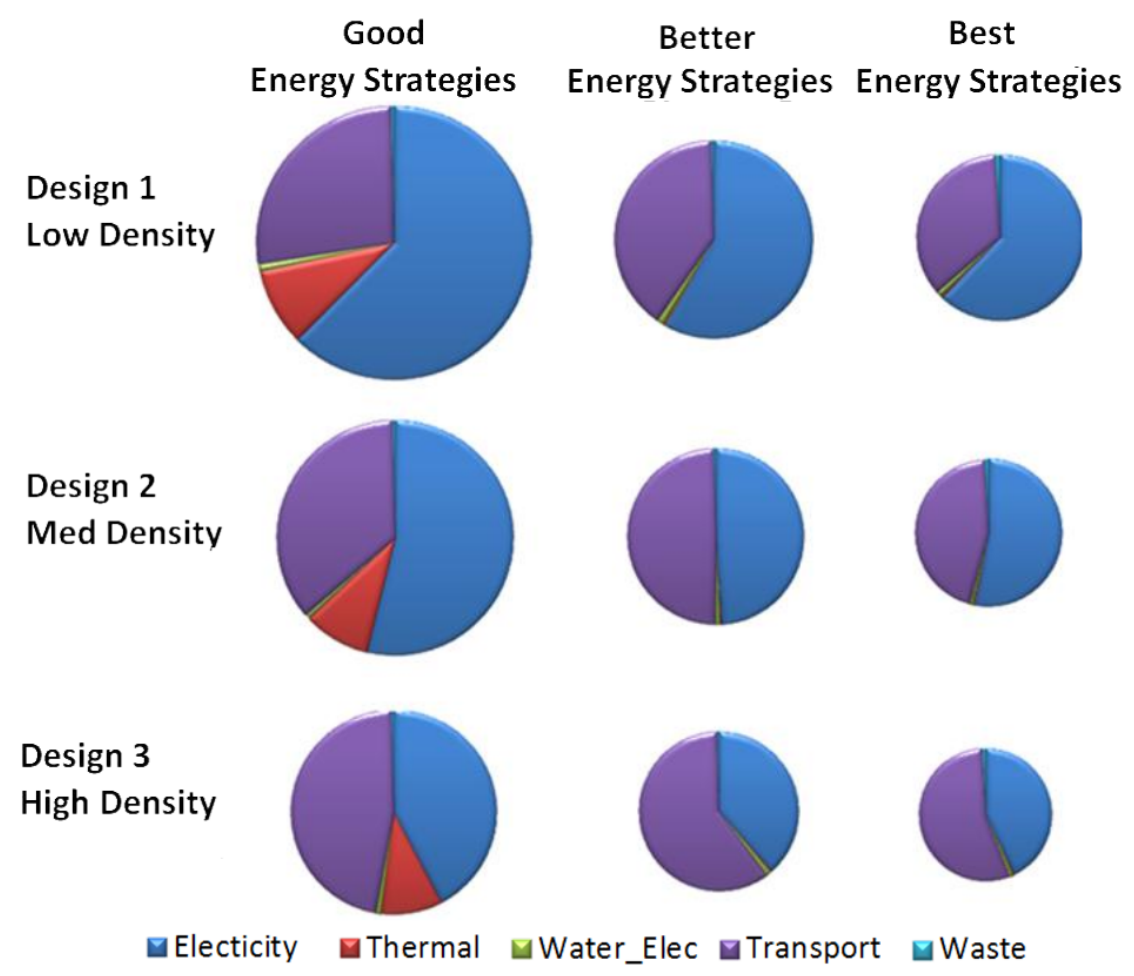

Figure 4.4: Comparison of sources of annual per capita carbon emissions 3 designs under 3 energy scenarios. HierSynth automation enabled this figure to be produced in under ten minutes compared with several hours work previously. 
masterplanning process.

Having shown HierSynth can help reduce the gap between the analysis and design cycle we then discussed whether or not HierSynth could be used to improve current modelling capacities.

\subsection{Analysis over Time}

Having automated current practice by integrating each model into HierSynth and so provided a faster way to run an analysis cycle on multiple designs under multiple energy scenarios we then looked at how to improve on current practice by using the capacities given by a computational framework. Our first objective was to enable analysis over time.

Arup's IRM model is constructed to assess a development at a particular point in time under a particular set of assumptions about building codes, emission factors and resource demands. Many of these factors will vary over time for example as building codes are tightened toward "zero carbon" development. Modelling these trends is currently beyond the scope of the model.

With HierSynth we looked to address this. As a first step we wanted to model the development at different phases of construction as different districts are developed or redeveloped over time. In CommunityB we analysed the development over 10 construction phases each lasting a few years with different districts being developed or redeveloped in different construction phases. This enabled us again to automate a time consuming manual process of enabling and disabling districts within the IRM model to manually compute KPIs for different construction phases. This enabled vastly improved analysis time particularly when performed across the nine composed scenarios discussed in section 4.6 (p148).

To enable analysis over different construction phases within HierSynth we implemented each phase as a different base scenario. This meant that a given execution chain would be executed for each of the base variable mappings chosen, enabling up to 90 different scenarios to be analysed $(3 \times 3 \times 10)$. The base variable mapping being patched by any scenario generation trees and then used in any analysis trees in the execution chain.

In practice the different construction phases were implemented as different columns of an excel table with the rows being different variables of the base variable mapping. An example portion of this phasing table is shown in in Fig. 4.5 (p154).

The results of introducing analysis over time are shown in Fig. 4.6 (p155). This form of figure would previously have taken a several hours of work and can now be generated with 10 model runs and 5 minutes computation. This figure shows us not only the growth in the size of the development, but the way that energy efficiency measures will 


\begin{tabular}{|l|l|l|r|r|r|}
\hline Name & \multicolumn{1}{|l}{ Sheet } & Cell & Phase1 & Phase2 & \multicolumn{1}{c|}{ Phase3 } \\
\hline Control_Er1_DayTimePlugLoadReduction_Sc1 & Control Panel & K7 & 0.007 & 0.008 & 0.010 \\
\hline Control_Er1_NightTimePlugLoadReduction_Sc1 & Control Panel & K8 & 0.039 & 0.043 & 0.048 \\
\hline Control_Er1_NarrowFormGuides_Sc1 & Control Panel & K9 & 0.100 & 0.100 & 0.100 \\
\hline Control_Er2_NoAC_Sc1 & Control Panel & K10 & 0.000 & 0.000 & 0.000 \\
\hline Control_Er3_HydronicCooling_Sc1 & Control Panel & K13 & 0.032 & 0.033 & 0.035 \\
\hline Control_Er3_ServerEfficiency_Sc1 & Control Panel & K14 & 0.007 & 0.008 & 0.010 \\
\hline Control_Er3_Other_Sc1 & Control Panel & K15 & 0.063 & 0.066 & 0.070 \\
\hline Control_Er4_ExistingEfficiency_Sc1 & Control Panel & K17 & 0.004 & 0.005 & 0.007 \\
\hline Control_Er4_NewBuildingsEfficiency_Sc1 & Control Panel & K18 & 0.095 & 0.100 & 0.105 \\
\hline
\end{tabular}

Figure 4.5: The data table representing the Phasing table which was used to add time as a dimension to the IRM model which previously only analysed at a fixed point in time. Changes in energy efficiency strategies were modelled over construction phases, for example modelling increases in server efficiency over the 10-20 years covered in the construction phases.

tend to reduce carbon emissions over time for example notice the decease in carbon emissions between phases five and six when little building work occurs. Critically we can see how moving from a business as usual energy strategy to a "Better" or "Best" strategy will lead to near constant carbon consumption despite substantially increasing population.

Creating a phasing table also enabled us to model changes in assumptions and energy efficiency strategies over time by varying the corresponding inputs to the IRM model. This enabled us to see trends over time and to model possible futures such as the introduction of more stringent building codes and more effective appliances and building materials. Some of the assumptions modelled over time are shown in Fig. 4.5 (p154) along with the cells in the model they will be entered into. This detailed modelling over time produced more accurate analysis over time such as is shown in Fig. 4.6 (p155).

Another set of results from adding construction phases to the model are shown in Fig 4.7 (p156) which also breaks down the analysis from development level by district and by the existing and new-build development to occur in that district. This was achieved by swapping the development analysis tree in the execution chain for one which broke the development up by district. This gives a previously unavailable level of detail into the design. It enables quick identification of poorly performing districts and anomalous results. It also shows the impact of trends over time predicted by the team on individual districts. This level of detail can be generated for each of the design / energy scenario pairs shown in Fig 4.4 (p152) which identified which design scenarios would be impacted most by predicted futures. For example examining the effectiveness of encouraging the uptake of electric cars in reducing overall percapita carbon emissions. Here we see that a greater relative effect would be had under a more dense design under the better or best energy scenario since in these cases the transportation component of carbon 


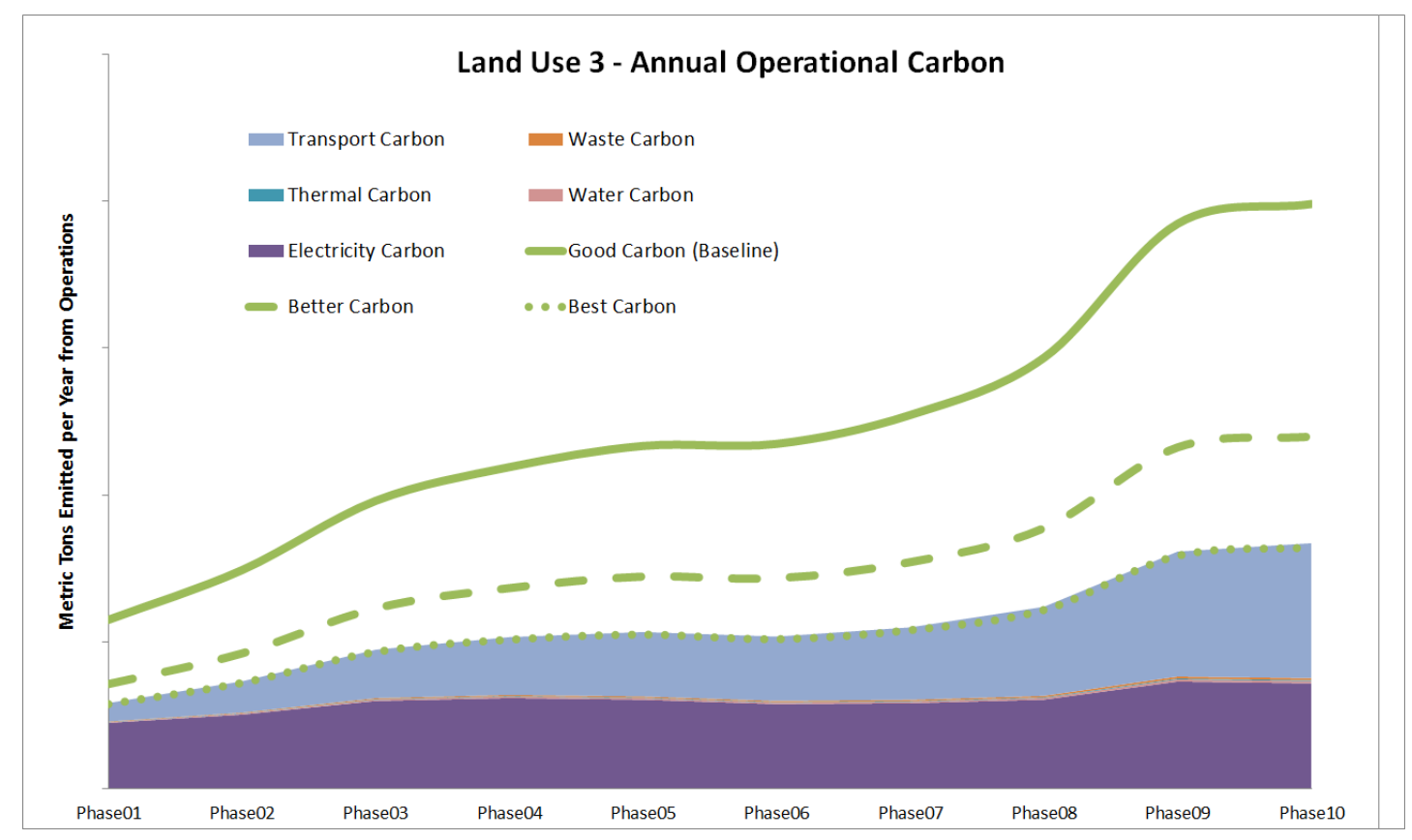

Figure 4.6: Annual operational carbon emissions over 10 construction phases for a high density design under the "Good", "Better" and "Best" energy strategies. Graphing over time was enabled via HierSynth integration, this graph also includes varying assumptions about building codes, emissions factors and energy efficiency over time adding a valuable accuracy and foresight capacity to the model. 


\section{Per Capita Carbon \\ Per District \& Construction Phase}

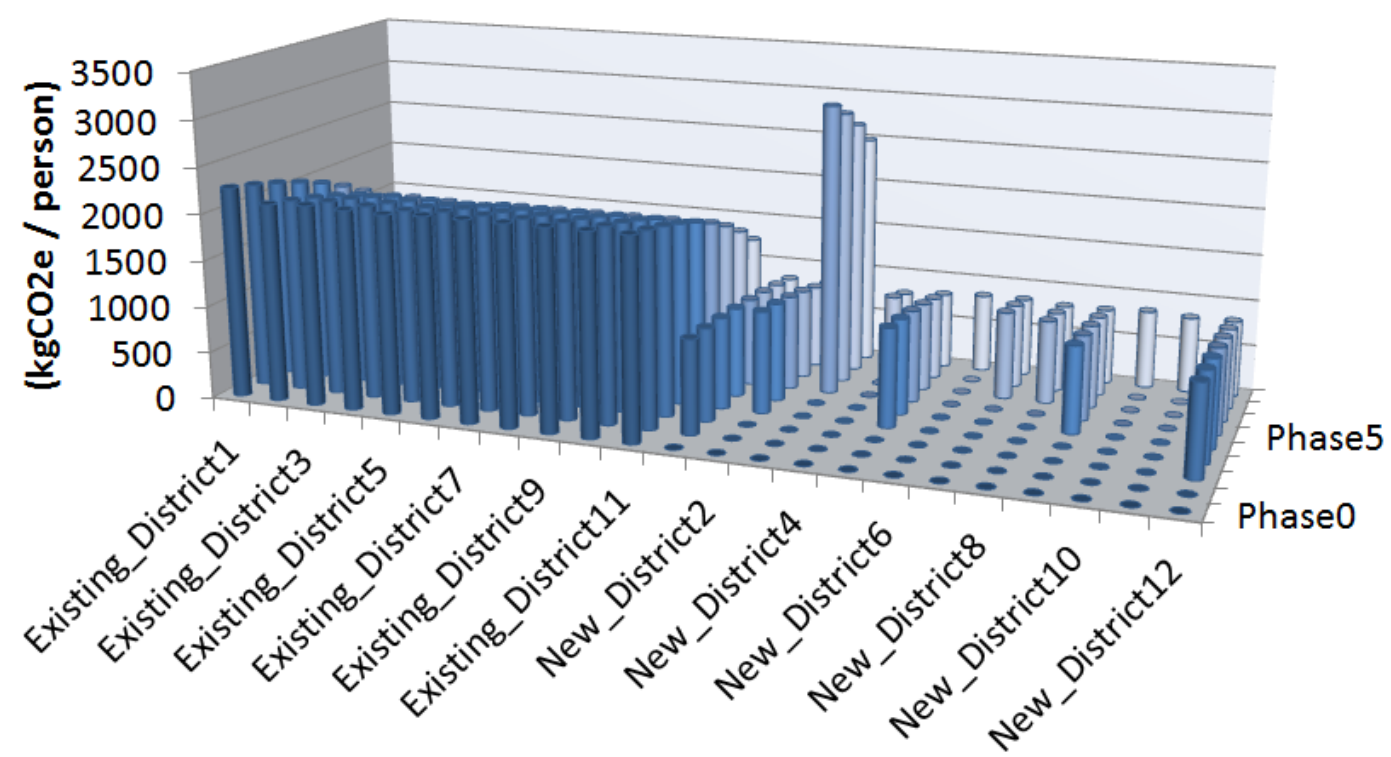

Figure 4.7: Annual percapita carbon emissions plotted over time and per district (existing and new). HierSynth enabled modelling of different construction phases and changes in assumptions (e.g. appliance efficiency) over time. This identified trends and coupled with increased detail spotted anomalies such as the one shown here. 


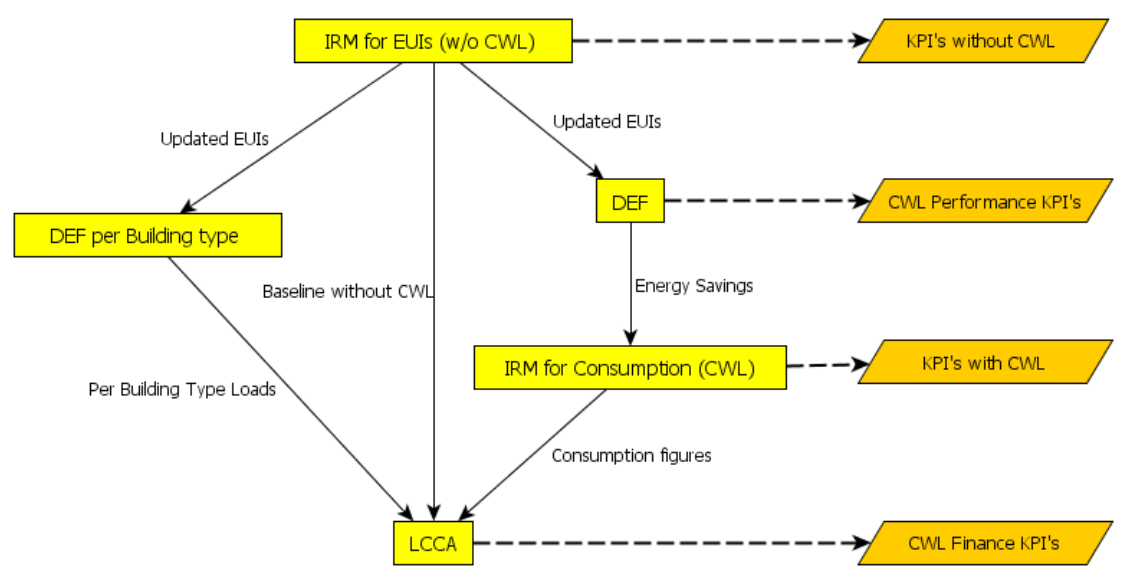

Figure 4.8: Conceptual model of data flow between the three Arup models integrated using HierSynth. Integration is discussed in section 4.9 (p157): the IRM model computes Energy Use Intensities (EUIs) for the design without a district energy system, the DEF model then uses these to size several forms of district energy system (e.g. Condenser Water Loop) and the LCCA model estimates the financial cost of the system. From every model HierSynth extracts a set of KPIs as shown by dashed lines.

emissions is a higher percentage.

Another positive outcome of adding this level of detail to the results generated was the number of bugs that were identified and fixed by using the more detailed insight. Most common amongst these were problems surrounding proportioning of development wide resource consumption or carbon offset to single districts which arose during the process of automating the model. Several times percapita carbon emissions were found to be negative which was traced to have been caused by a carbon offset originally entered as applying to the whole development not being scaled down when only a single district was under consideration. Similarly certain districts with unusual concentrations of certain landuse types identified previously unseen behaviour in the model.

Having demonstrated the value of a computational framework being applied to a single model the second phase of the collaboration was to consider the automation and then the integration of several models.

\subsection{Integration}

As discussed in section 4.4 (p144) there is a natural flow of analysis between the IRM, DEF and LCCA models, hence we used HierSynth to integrate these models into a larger analysis module able to produce more accurate and detailed results whilst exploring design options previously not possible without a great deal of manual work. During this integration process we were able to improve the quality and robustness of each of 
the models.

At a high level Fig. 4.8 (p157) shows the data flow between these three models, each of which was developed separately by different practitioners. First the IRM model computes energy use intensities for the land use types in the design. These reflect the demand for electricity, heating and cooling in $\mathrm{kwh} / \mathrm{sf} /$ annum per land use type. The DEF model then uses these to size several forms of district energy system taking into account expected usage patterns and weather data to model load and consumption over the year. This district energy system is then entered into the IRM model producing an analysis run showing its effects on many KPIs. Finally the LCCA model estimates the financial cost of the district energy system based on estimates from previous projects.

This flow of data was generally undertaken by different practitioners running their models to compute results which were sent to the next practitioner to input into their model. This process illustrates the challenges identified in section 5.2 (p211), namely the length of time the analysis took, the challenges of cross disciplinary communication and a lack of scalability. To address these and to show the value of a computational framework designed to meet these challenges we used HierSynth to integrate these three models together into a larger analysis module.

There were several benefits to the analysis cycle from this integration. Firstly automation and integration substantially speeds up a slow manual process and enabled more detailed analysis under more scenarios than was previously feasible in the project. The main benefit of the integrated model is that it enabled investigation of the effect of changes in one model in the others. Most notably we were able to consider the effects different energy strategies in the IRM model upon the district energy system given by the DEF model and its costing given by the LCCA model.

Another benefit was that since both the IRM and DEF models calculate annual electricity and gas consumption the integration of these two tools led to an opportunity to verify the models against each other. Both models used different calculation methods, for example the DEF model included weather trends whilst the IRM didn't but did include a more detailed breakdown of sources of energy demand (lighting, appliances and so forth). After a number of model and integration issues were resolved we were able verify that the models were agreeing to better than $\pm 5 \%$. However since the IRM model involves more detailed modelling of energy demand and supply it should be more accurate and so it has been used for most of the results generated through this chapter.

The process of integration was not straightforward as these models had not been designed in conjunction with one another, some of the challenges encountered are discussed in section 4.9 .2 (p161), however resolving these challenges has improved the quality and robustness of the models and their results. 


\subsubsection{Implementation}

As discussed in section 4.4 (p144) the three models to be integrated together are implemented as complex spreadsheets containing up to 50,000 calculation cells. Each was developed by different practitioners with different aims and they were not built to be integrated together. Each model was integrated into HierSynth as a differently configured Excel analysis node with a set of inputs and outputs. These nodes were then duplicated and configured to compute different parts of the analysis workflow. In total an ensemble of 11 different models performing different types of analysis were used in conjunction.

Fig. 4.9 (p160) shows the full data flow diagram between all of the models. Key features of this integration are:

- The flow of information from IRM to DEF to size a district energy system based on IRM calculated EUI's.

- The flow of information from DEF back to IRM to update the analysis by including a district energy system in the analysis.

- The use of two IRM models to produce a final sustainability assessment with and without a Condenser Water Loop (CWL) district energy system for comparison.

- The use of a set of DEF models to calculate the gas and electricity loads for each building type since this break down was not given in the DEF model but was required in the LCCA model.

- Finally the use of the LCCA model to cost the proposed district energy system which uses inputs from the per building type DEF models and the final pair of IRM models.

- At all stages model KPIs were extracted for comparison and validation purposes.

Each of these model nodes was created and configured in the HierSynth tree shown in Fig. 4.10 (p161), The set of models are run using linked executions enabling the analyses to run in the correct order and to allow access to outputs of prerequisite calculations. This analysis tree could then be combined with a number of scenario generation trees to analyse multiple designs under many scenarios and also to investigate a number of insight questions posed by the team which are discussed in section 4.12 (p171). First however we will discuss some of the challenges encountered in the construction of this model ensemble. 


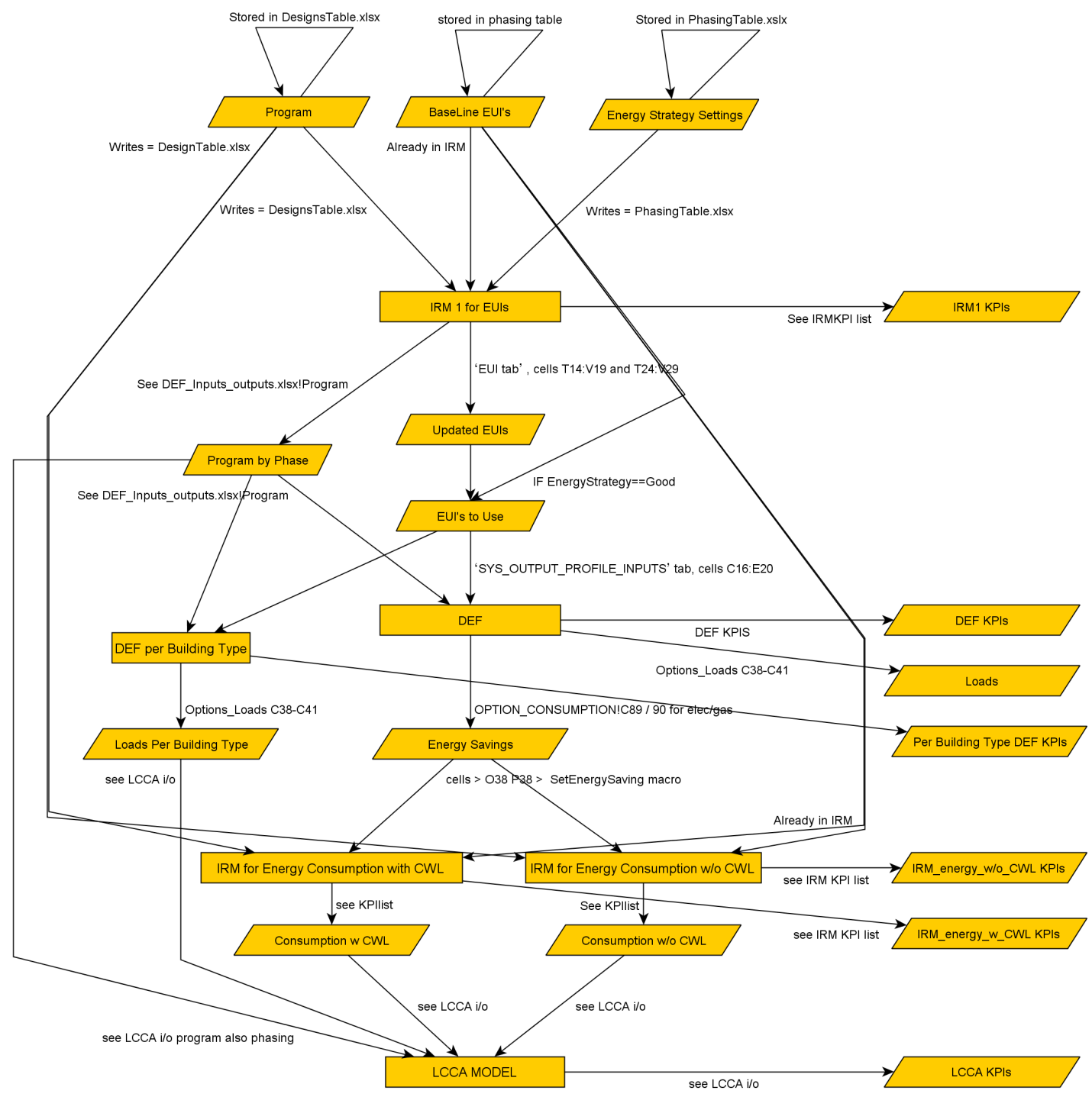

Figure 4.9: The full data flow diagram of how the integration of the IRM, DEF and LCCA models works. This data flow model is implemented in the HierSynth tree shown in Fig. 4.10 (p161). 


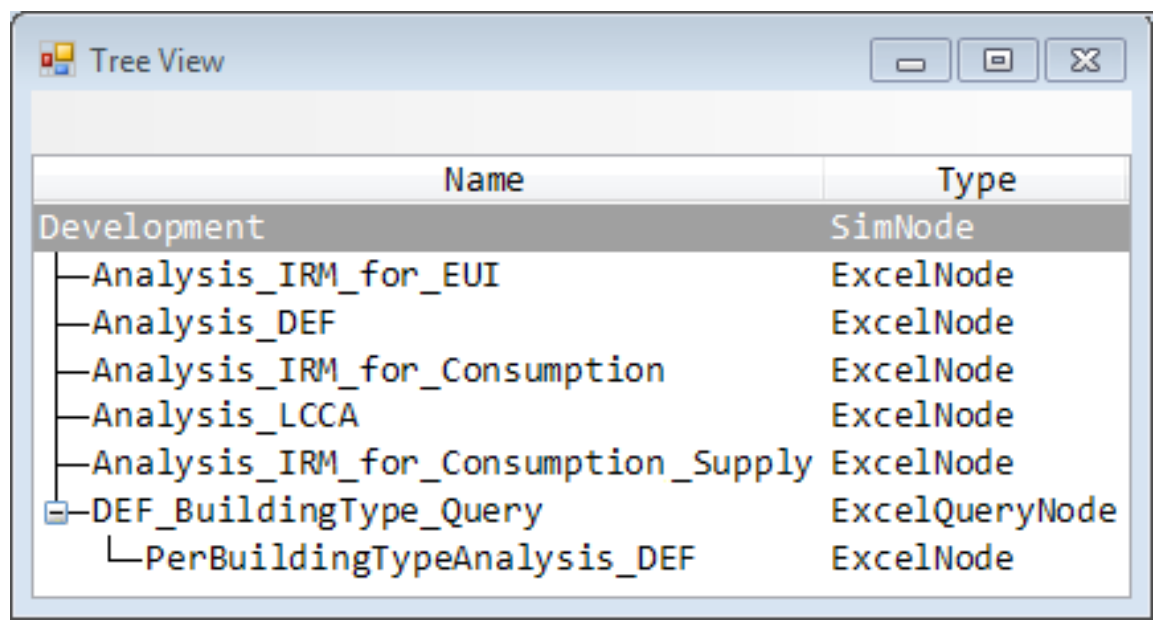

Figure 4.10: HierSynth tree template used to implement the integration of models sketched in Fig. 4.8 (p157). This analysis tree was reused to answer many different questions and was used to analyse both the whole development and each district.

\subsubsection{Challenges}

Perhaps not surprisingly the integration of these three disparate models was a challenging process and during the construction and validation of the integrated model ensemble a number of bugs were identified and fixed.

Given the challenges inherent within spreadsheet based modelling discussed in chapter 5 (p209). As motivation for the resolution of such challenges we record examples of the types of bugs generated or encountered. Some of these were pre-existing in the models however many were introduced during the integration process. The fixing of these problems produced more robust and accurate models.

- Enabling the IRM and DEF models to produce mutually consistent estimates of resource demands involved identifying a number of small calculation bugs.

- Initially the IRM and DEF models worked with a different fixed number of construction phases and the DEF model has to be reworked to accommodate a higher number of phases.

- Similarly the DEF and IRM models had a different breakdown of the landuse schedule into building types which had to be unified.

- Two of the models required calendar years to be associated with the end of each construction phase, doing so consistently presented a challenge as one model required years to be consecutive.

- Simple bugs encountered included a failure to convert from $M^{2}$ to sqft, one model 
requiring a cumulative tally of built Gross Floor Area (GFA) and another requiring the amount built in each year.

- Some difficulty was encountered with an optimisation macro within one of the models not being sufficiently robust to deal with the range of scenarios the analysis models were run with. Indeed this was a common theme, that as we automated then integrated the models we found more scope to explore the design space and undertake "what if" analyses and in doing so we exercised areas of the models more robustly than previous manual use and on several occasions found limitations in the models which were then fixed.

Over the course of this integration phase over 30 iterations of the models were created until we were able to sign off a correctly functioning integrated model ensemble. This approval was achieved by manually ensuring that calculated KPIs were within the ranges expected expected by practitioners. This was done for several dozen KPIs across over around 600 model runs using graphs similar to 4.7 (p156) to ensure that the model was accurate when using different designs, districts, construction phases and energy strategies. This model chain was then used repeatedly to produce insight for the design and an analysis teams.

\subsection{Integrated Results}

Having produced and tested this integrated model chain, it was applied to analyse a large number of designs and scenarios to answer a number of insight questions the team proposed. Examples of the value of the integrated model are given in the next few figures. As well as speeding up analysis the value of an integrated model was threefold: Firstly providing substantially more detailed analysis results enabled focusing on problem parts of the design. Secondly the combined capacity of the models together with HierSynth enabled more accurate analysis capacity not previously possible, such as the sensitivity analysis discussed in section 4.13 (p187). Finally and perhaps most importantly the system enabled design space exploration in a way not previously possible. The results of such explorations are discussed in section 4.12 (p171). First however we will look at a selection of the many analysis results produced using HierSynth and the integrated model.

Fig. 4.11 (p163) displays a geolocated per district mapping of analysis results under the nine different design scenarios shown in Fig. 4.4 (p152). This was produced by combining the scenario generation tree discussed in section 4.6 (p148) with an analysis tree that queried each design for its constituent districts and then used the integrated analysis module to analyse each district. Analysis was also run at the development level and is shown in the bottom left graph demonstrating the value of computing at 


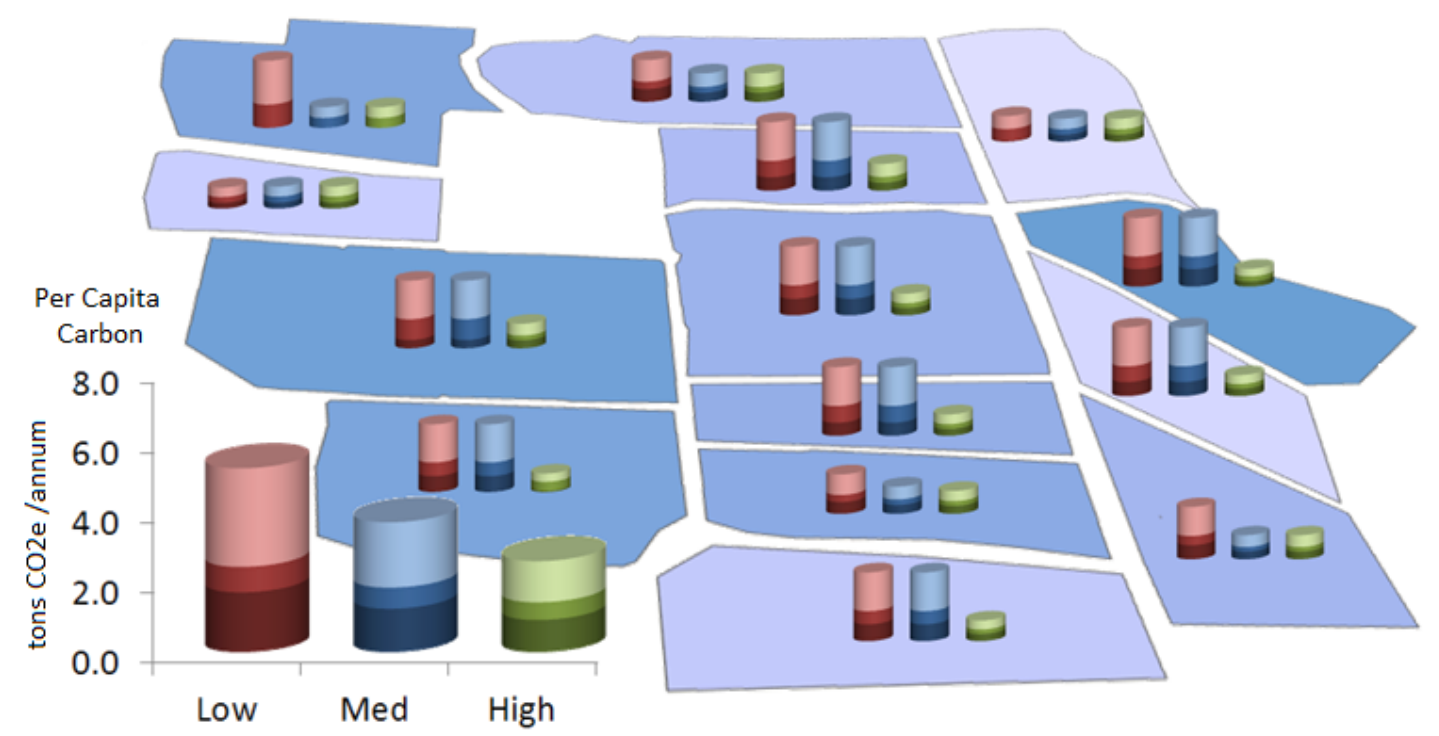

Figure 4.11: A per district percapita carbon map for 3 designs under 3 energy scenarios ("Good" is full column, "Better" is middle marker and "Best" is lowest marker). HierSynth automation enabled more detailed insight at the district level to be computed for each design iteration. Without this only the bottom left graph would have been computed which shows results for the whole development.

multiple design scales.

Previously the level of detail displayed in Fig. 4.11 (p163) was not available. However with it, it is possible to use this more accurate model ensemble to gain insight into which districts are performing well for many different KPIs. As an example consider that not all districts were developed or redeveloped under different designs. Consider the two districts in the top left, the lower one is a new build district and provides good carbon performance over all designs / scenarios, whereas the top left district performs poorly until it is rebuilt under the Medium and High density designs. This figure shows carbon emissions percapita but other figures were produced showing any of the model KPIs, for example electricity and gas consumption.

The results of such an IRM analyses were then automatically used in a DEF analysis enabling comparison of different district energy systems using a variety of KPIs. For example Fig. 4.12 (p164) quantifies the impact of a district energy system upon the annual energy consumption of the development under nine scenarios (we note that the "Good" scenario does not have a district energy system). This enables us to identify an increasing fall in energy consumption under the "better" and "best" energy scenarios. Note that population increases substantially between each design yet energy consumption does not show a proportional increase. Instead the increasing density of the design enables better energy strategies and improves the efficiency of others 


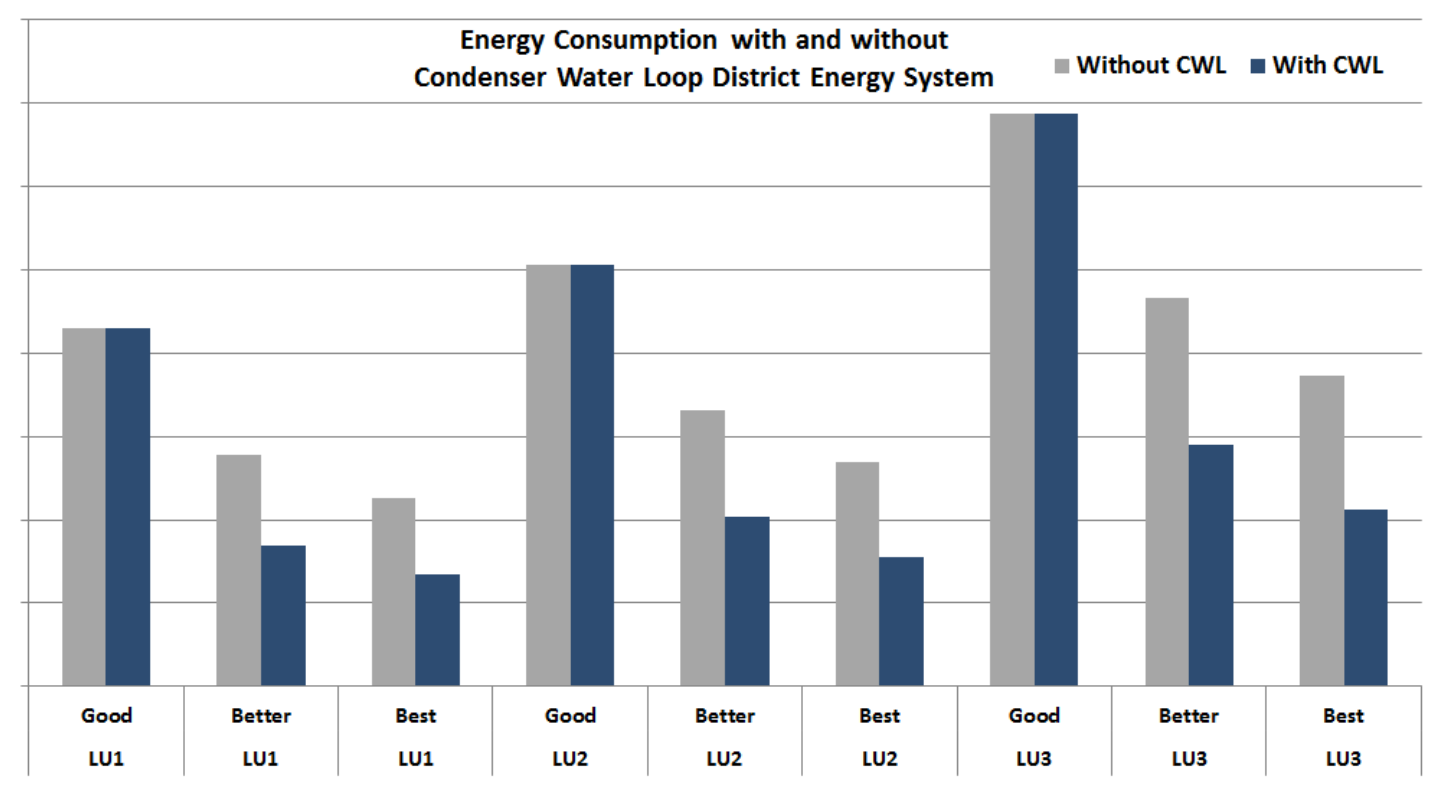

Figure 4.12: DEF energy consumption figures (combined gas and electricity) as calculated from inputs from IRM model under nine design scenarios. Note that the "Good" scenario does not include a district energy system. Scale removed to preserve confidentiality.

(such as this district energy system) which result in only modest increase in energy consumption.

Finally integrating the LCCA model allows us to consider the financial performance of a district energy system. Using inputs from the DEF and IRM models the LCCA model can put a financial cost to a proposed district energy system. As an example Fig. 4.13 (p165) shows the Internal Rate of Return (IRR) for investing in two possible district energy systems under the nine scenarios discussed in section 4.6 (p148). Note the increasing cost effectiveness of the investment as the density of the development increases. This quantification across many more scenarios is very valuable to the design team.

In conclusion the automation and integration of these three models using the HierSynth platform with its scenario generation and integration capacities has provided several benefits to the analysis team. By giving more detailed and more accurate insight into the design under more of scenarios than previously possible. Integrating the models together also enabled the analysis team to work together more closely as their models were used in concert to explore scenarios not previously possible. Over the course of the collaboration multiple model bugs were identified using HierSynth and fixed resulting in more accurate and robust models. The results explored in this chapter shows just a few of the insights generated using HierSynth. Over the course of the collaboration we analysed over 13 iterations of the design or models providing more 


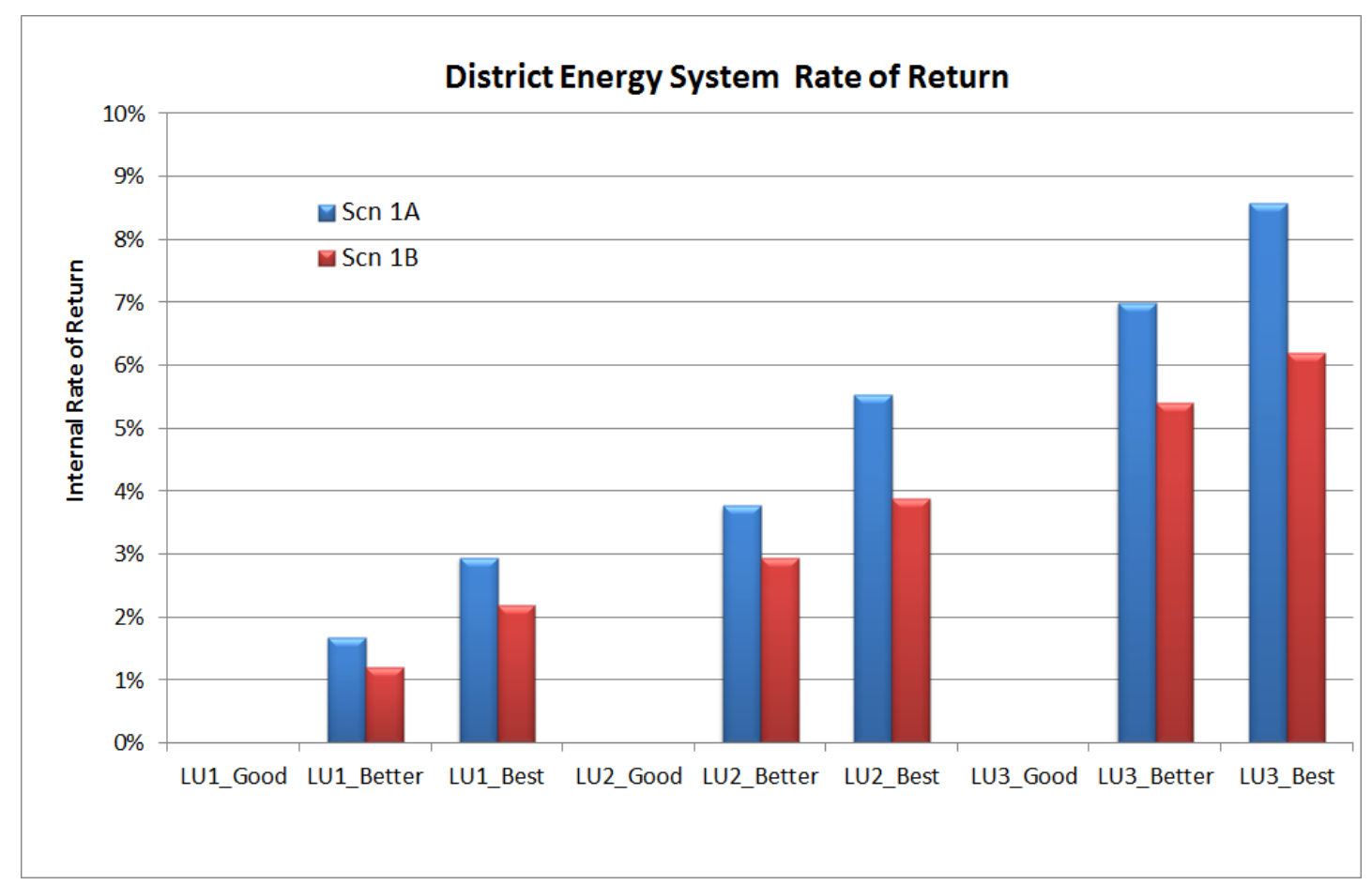

Figure 4.13: LCCA results showing Internal Rate of Return (IRR) for three designs under three energy scenarios. Results are calculated based on inputs from the IRM and DEF analysis models. Note that the "Good" scenario does not include a district energy system. Two implementations of district energy system are used for calculations, $1 \mathrm{~A}$ is a Condenser Water Loop and 1B adds a Thermal Energy Storage capacity to the system to store excess heat to meet peak demands avoiding investment in expensive additional generation capacity. 
detailed results more quickly than previously possible. This integrated model was then used as the basis of an investigation into the design space around one of the proposed designs. This will be discussed in detail in section 4.12 (p171).

"Overall HierSynth has quickly generated results in lists and charts that allow evaluation of results across a number of models and districts that would not be possible to generate in the time allowed for the project, otherwise." Design Team Member, Buildings Sustainability Group, Arup

\subsection{Block-level Analysis}

Thus far we have discussed non-geometric analyses that do not take into account the building's actual shape. In part this is due to the early stage of the masterplanning project where detailed design has not yet been undertaken. However having demonstrated the benefits of increasing the level of detail from development to districts (e.g. Fig. 4.11 (p163)) we explored the options for city block level analysis.

\subsubsection{Lighting Analysis}

During the masterplanning phase the geometry provided is not final building designs but is building envelopes which demonstrate the maximum size of each building in height, width, set back from the road. Internal parameters such as the number of stories are set along with the expected floor space mix per block or per building. The final design of these building envelopes is likely to be subcontracted to other design firms who must meet the design parameters set by the urban masterplanning team. These parameters would include maximum height, number of stories, total floor space, mix of land use types as well as conditions to create a consistent look and feel across the development.

Finally targets would be set for energy use intensities and emissions targets to be met by the design. In many cases the setting of these energy and sustainability targets will be set by or in collaboration with the analysis team. At present these targets are generally set for the whole development using development wide analysis tools.

In contrast to this we used the HierSynth framework to apply a number of analyses at a per city block and per district level using geometry received from the design team. Our first step was to analyse the entire development using a lighting analysis.

As discussed in section 4.4.1 (p144) another of the models we used to analyse the masterplan designs was Radiance [103]. Radiance is an open source lighting simulator in wide use across the industry. Whilst not previously used on this project it had been previously integrated into HierSynth and was applied to the project geometry we received. 


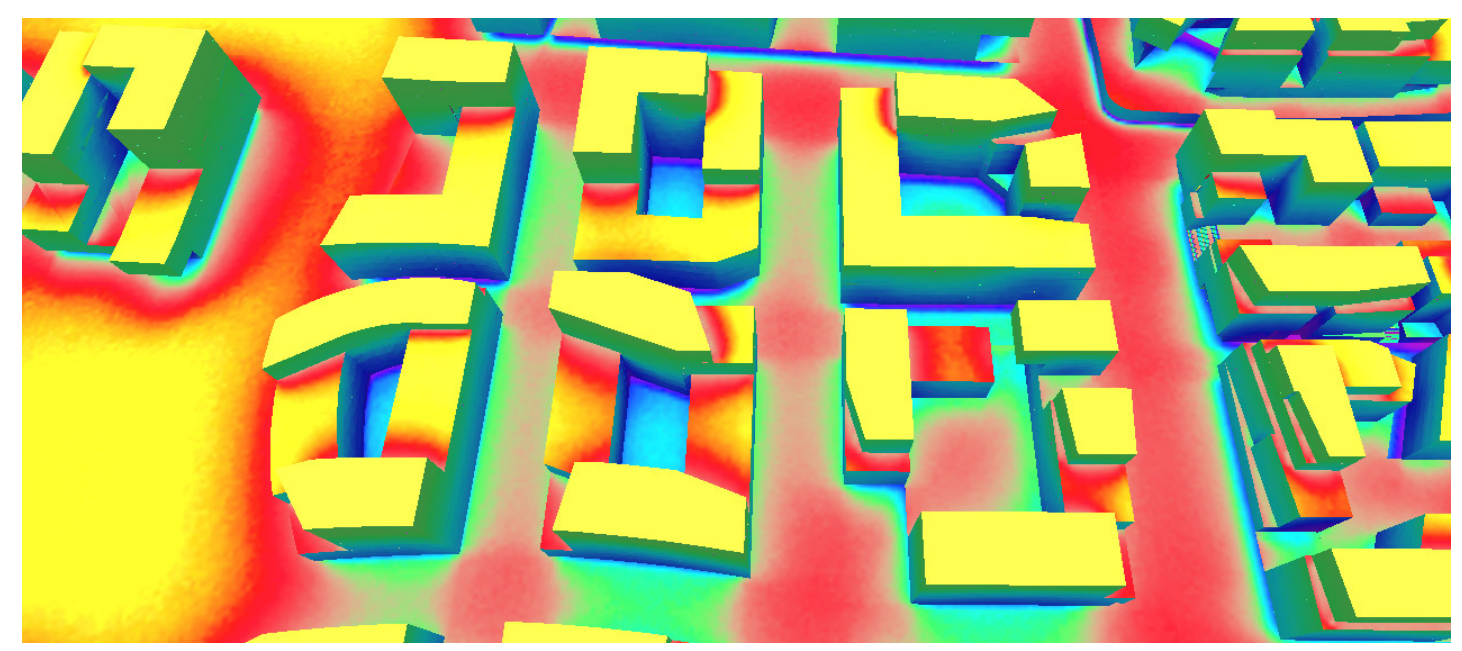

Figure 4.14: Automated BRE209 [63] daylight analysis of sample geometry for CommunityB project computed using Radiance [103] automated by HierSynth. A false colour scale is used to show illuminance with yellow being full sunlight and dark blue being none. Notice the difference between buildings courtyards as the density of the building changes.

After preprocessing the geometry received from the design team to remove excess geometry and simplify the model HierSynth was used to run a day light simulation and automatically produce a $3 \mathrm{~d}$ fly through environment which can be seen in Fig. 4.14 (p167). In this case a BRE209 [63] analysis was used which computes illuminance (incident sunlight over a surface weighted by the human perception of brightness) under a standard 10,000lux overcast sky. A false colour scale is used to highlight results, from yellow being full sunlight down to dark blue being zero sunlight. This form of analysis enables the design team to get a feel for how the design and density of the development will affect the feel of the environment. For example whether the streets are too narrow? (e.g. the horizontal street in the middle of Fig. 4.14 (p167)). Also of interest is how different densities of development result in different forms of buildings with different light characteristics. Consider the six city buildings shown in this figure, the top left and bottom right are lower density compared to the other four. As a result these buildings have well lit (red/green) spacious courtyards and walls which are mainly green/red indicating they receive sufficient lighting. On the other hand the remaining four buildings have deep and dark courtyards as indicated by the dark blue colour. Another use for these analysis results is in the assessment of rights to light, overshadowing and the potential for solar panels.

Interactive visualisation like this using a commercial games engine Unity3d [100] enable detailed investigation of the performance of the design and are good communication tools for discussion with stakeholders and potentially the public, particularly as the Unity3d engine can be embedded on a website. This visualisation is specially 
useful when it composes a number of disciplines results together into one visualisation as we will now discuss.

\subsubsection{Block Performance}

In order to integrate the physical geometry of the development with its sustainability performance we undertook a case study analysis of one district of the CommunityB development. We aimed to analyse each city block of the model using Arup's IRM model to assess energy and water demand along with carbon emissions. Having chosen a case study district we were unfortunately unable to obtain per building land use schedules mostly due to the early nature of this project. Instead these were simulated by heuristically apportioning the total landuse mix of the district under consideration between the city block according to the total floor area of each building as calculated from the geometry shown in Fig. 4.14 (p167).

This block level landuse plan formed the input data for a set of IRM analysis using HierSynth. Input data for each city block (which might be composed of one or a handful of buildings) consisted of the number of stories, expected population, gross floor area (GFA) mix between six types of building (housing, existing office, new offices and so forth). These were then automatically entered into an IRM model to calculate energy and water demand as well as carbon emissions and other KPIs. The DEF and LCCA models could not be deployed at this scale as a single building is too small for a district energy system. Normally the IRM model is not applied at this building scale hence the results are a little coarse and a number of model problems were identified and fixed to cope with smaller scale projects. HierSynth was then used to automatically visualise geolocated results in the Unity3d environment.

Fig. 4.15 (p169) shows the geolocated results of such an analysis. Combined with a daylight analysis shown on a greyscale colour scheme is a series of billboards showing on a red,yellow,green colour scale the water consumption per person per day for each building. This highlights comparatively poorly performing buildings, for example housing and the local fitness centre score badly as do existing office buildings. This analysis and visualisation system enables multiple KPIs to be visualised and explored interactively enabling quick comparison between them. Each block also has a more detailed information display which can be seen on closer inspection.

Fig. 4.16 (p170) shows this detailed block information. What is being visualised here is a performance tree for each building as calculated during the aggregation stage of HierSynth tree execution. (see sections 2.2 (p70), 3.7 .3 (p108) and 3.8 (p108)).

These visualisations show two things. Firstly the top bar of each board shows the name of the block and highlights score for a KPI on a red / yellow / green colour scale relative to its peers (here an overall score). Below this is a more detailed performance tree which shows a number of KPIs and their composition. In this case three metrics 


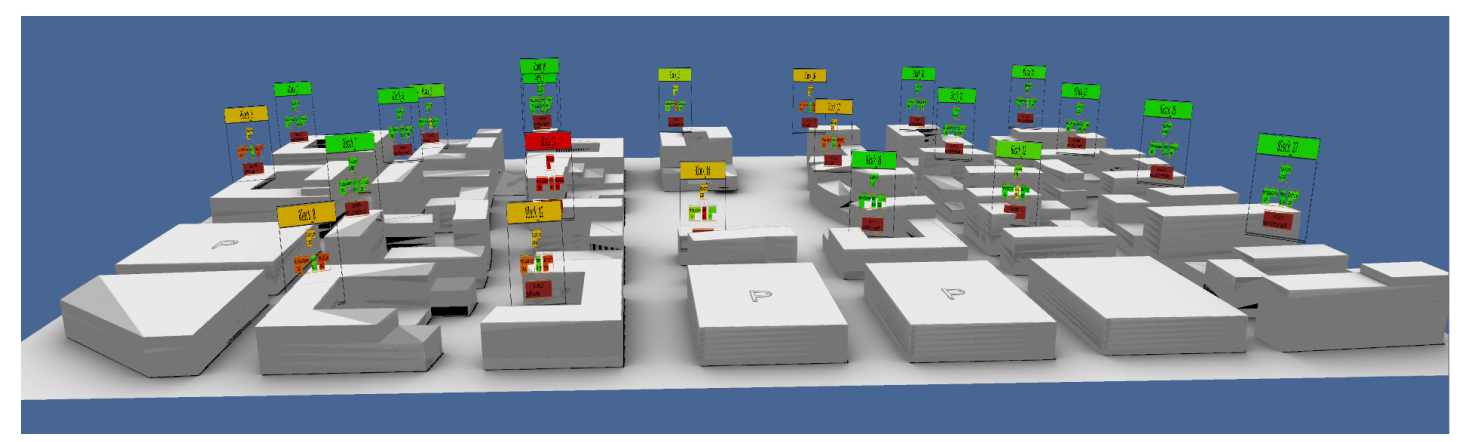

Figure 4.15: Geometry with BRE209 [63] daylight analysis mapped to grey scale. Block level billboards display information about the sustainability performance of each city block. Interactive exploration of this environment enables identification of problem buildings and performance trends, in this case a red / yellow / green colour scale is used to show water consumption per person per day.

(per capita carbon emissions, water consumption per person per day and percapita electricity consumption) are composed into a weighted average "score". We also see a description of the building showing its composition in terms of GFA mix. This enables immediate investigation of any performance problems in the district. In this case we see that the electricity and hence the percapita carbon figure is comparatively high and makes a good case for redeveloping or retrofitting this old office block.

HierSynth enables multiple performance trees to be calculated and to be changed between interactively in the visualisation environment (see section 3.8 (p108)). This enables focusing on different performance criteria or the consideration of performance under different scenarios. The performance tree visualisation enables quick diagnostic investigation of poor performance from the buildings score down to the particular discipline, KPI and analysis result that identifies the problem. KPIs from multiple disciplines in multiple units can be combined using this approach.

To conclude, we believe that analysis on this level is valuable for improving the masterplan and that visualisations such as these are important. The visualisations shown in this section were presented to the analysis and design teams and responses recorded during video calls. The concept of such visualisation was well received by both the design and analysis teams with particular benefit seen from the quantitative analysis results run per-building for the first time; particularly since it enabled focusing on poorly performing buildings. Similarly the ability to interactively compare the impact of different scenarios on different KPIs over a geometrical aspect made communication of results more effective across disciplines. However despite this potential the visualisations did not reach their full potential due to a lack of detailed data and geometry at this early design stage. This also meant that the impact of rapid quantitative visualisation could not be explored in this project. 


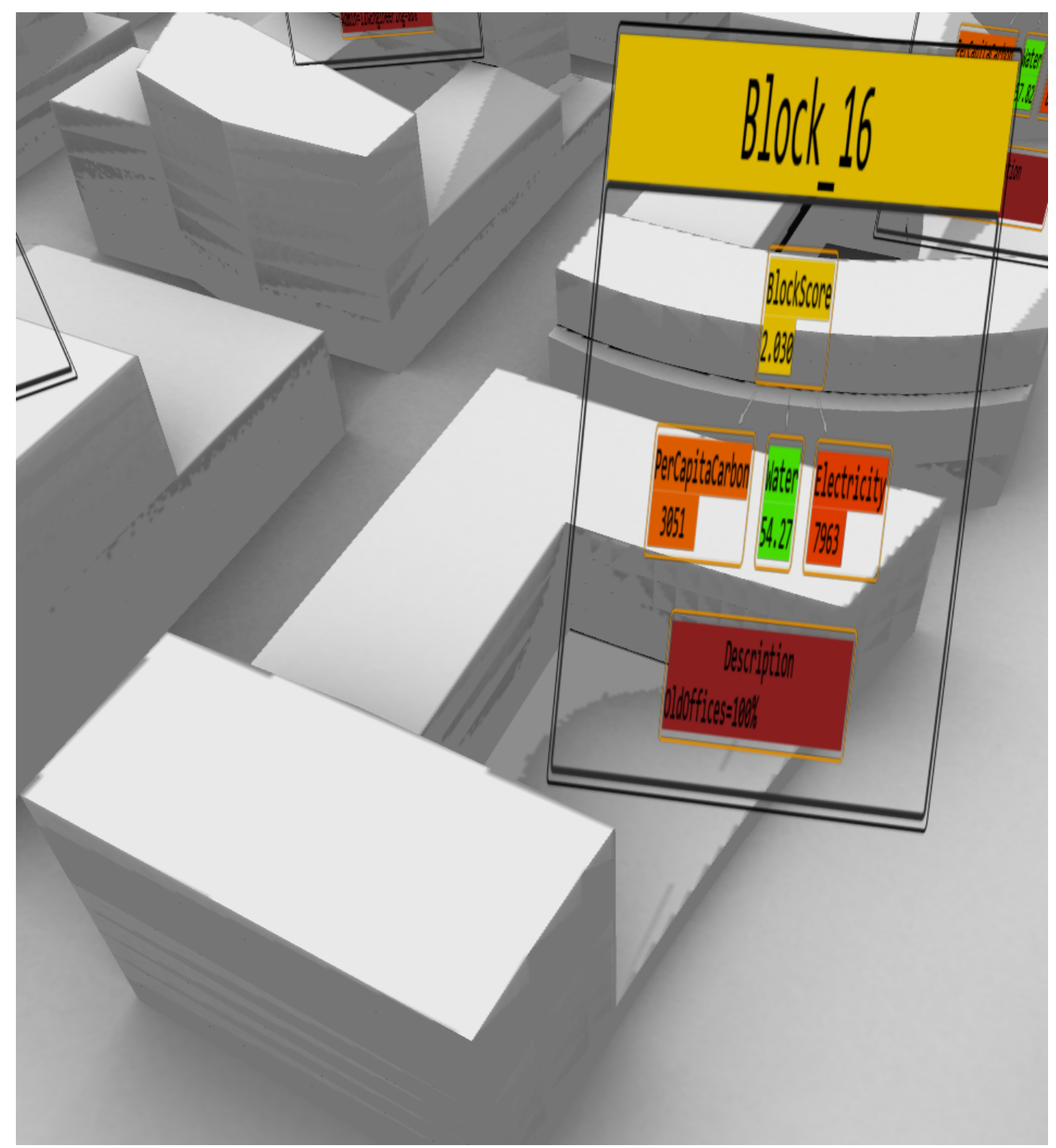

Figure 4.16: A performance tree for one of the blocks in this design. Whilst scoring well for water usage compared to its peers it performs poorly for carbon and electricity, which is not surprising as it consists entirely of old offices which have not been redeveloped in this design. Quickly identifying poorly performing buildings highlights areas for design improvement. 


\subsubsection{Further Work}

Some exploration was conducted into procedural geometry generation techniques to try to combat this problem however the results were not consistent with the standard of an architect's work. In our proof of concept applications of HierSynth we used GIS information as a source and automatically created correct geometry for analysis based upon the plot outline and the number of stories for each building envelope which was well received.

It is also important to say that whilst this analysis was based on a fairly simple set of parameters HierSynth would enable the inclusion of many more details about each building into the analysis had they been available for example the number of energy strategies (solar panels, low flush fixture and fittings ...) applied to each building.

Combining additional information about each building with additional analysis engines, particularly ones which take more account of geometrical form would also lead to more interesting results. Options for this would include analysing a building's energy performance using a model like EnergyPlus [101]. Other district wide geometric analyses such as wind flow or acoustic performance are also occasionally carried out on masterplanning projects and so could be integrated into HierSynth with an expectation of similar benefits to those shown in this casestudy.

Finally the analysis of multiple geometries for multiple designs would add an interesting dimension to this investigation allowing stakeholders to identify the effects of changes in geometry upon KPIs from multiple analysis engines and disciplines.

\subsection{Insight}

Having used the HierSynth platform to create an analysis platform targeted at the CommunityB development the scene was set to begin to investigate a number of "insight" questions. These questions were proposed by the analysis and design teams and take the form of investigations into the design space surrounding both the development design via its land use schedule and also the energy scenarios which are employed to help make the development as sustainable as possible. These investigations were explored using the models integrated into HierSynth and the design space explored using HierSynth's scenario generation tools alongside framework tools such as sensitivity analysis. Questions proposed were:

1. "What is the impact of electrical vehicle uptake on the performance of the masterplan area?" see section 4.12.1 (p172)

2. "What is the optimal (or critical) density for the district energy system?" see section $4.12 .2(\mathrm{p} 175)$ 
3. "What is the optimal mix of residential and commercial development for heat recovery and reduced vehicle miles travelled?" see section 4.12 .3 (p177)

4. "What are the development's carbon emissions most sensitive to? Which of these sensitivities can we positively impact?" see section 4.13 .1 (p187)

5. "How significant are the various (energy) strategies in reducing the developments carbon emissions and water intensity?" see section 4.13 .2 (p190)

6. "Is it better to vary density (i.e. build dense in a few areas and less dense in others), or build equal density everywhere?" This question was not tackled due to time constraints.

These investigations would not have been feasible to carry out within the project without the use of a computational framework such as a HierSynth. During these investigations many insights were gained into the development designs, the design space for those designs and the energy scenarios. We also gained some insight into the IRM model using sensitivity analysis and the tools discussed in chapter 5 (p209).

\subsubsection{Effect of Electric Vehicles}

What is the impact of electrical vehicle uptake on the performance of the masterplan area?

To undertake this investigation we looked at Arup's IRM model and used its transportation sub-model to vary the number of electric cars in the development. This was done by changing one of the energy strategies corresponding to the number of electric cars which in turn affected the transportation and energy demand models. From discussions with the transport planning team we decided to explore the impact of up to $20 \%$ electric cars as a proportion of the number of parking spaces in the development. The effects of these uptake levels would be considered across all KPIs from all models in the integrated model developed in section 4.9 (p157). Particularly expected to be of interest were effects on percapita carbon, particularly the transport component and any increase in electricty consumption used to fuel the electric cars. The effects would be evaluated over the 3 landuse plans of increasing density under the three energy scenarios.

This investigation is implemented in the execution chain shown in Fig. 4.17 (p173). For each of the construction phases considered (in this case just the final construction phase) this execution chain is executed. The first tree in the chain is the scenario generation tree from section 4.6 (p148) which creates variable maps for multiple designs 


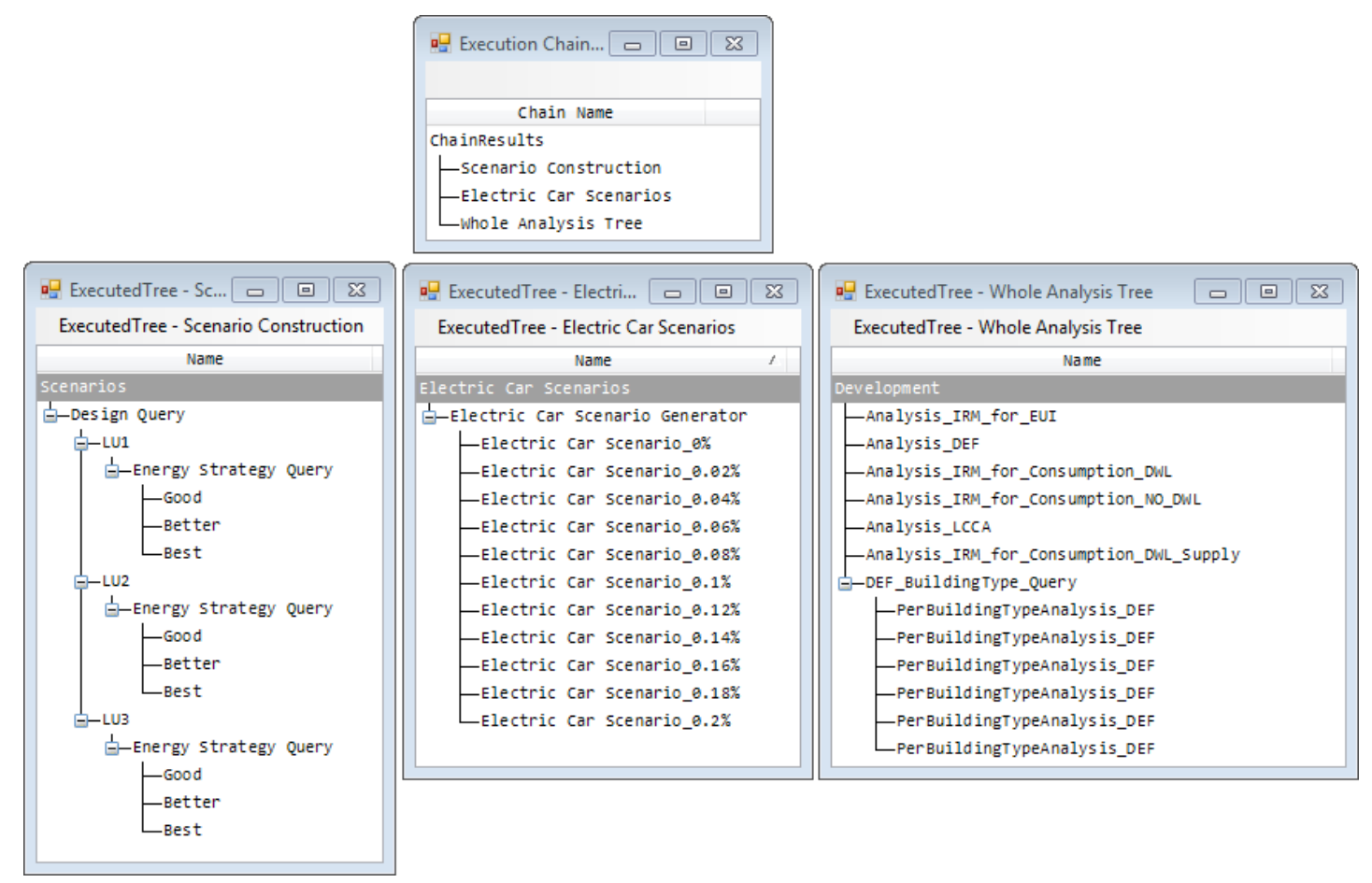

Figure 4.17: The execution chain used for investigating the effects of electric car uptake on the development. This tree produces 99 scenarios (11 uptake levels 3 designs and 3 energy scenarios).

under multiple energy scenarios. As shown by the number of leaf nodes in this first tree 9 design / scenario pairs will be generated. For each of these the second tree in the execution chain is executed and uses a generator node which acts as a loop to create a set of variable maps which are used to instantiate child template trees. In this case the loop iterates through different uptake levels of electric cars from $0 \% \ldots 20 \%$ in increments of $2 \%$. This is done by patching the energy scenario by amending the energy strategy for electric car ownership. Hence the second tree in the chain generates 11 new scenarios each time it is executed. Together these first two trees produce 99 analysis scenarios for examination. The analysis itself is conducted by the final tree in the execution chain shown in Fig. 4.17 (p173) which runs the integrated development wide analysis model discussed in section 4.9 (p157).

The results of executing these 1,188 analysis runs can be found in Fig. 4.18 (p174). The first finding was that under a given design scenario the models employed reported a constant relationship between the percentage increase in electric car ownership and effects upon operation carbon, transport carbon and electricity. Hence all of the results are reported for a given $1 \%$ increase in electric car ownership. For convenience only results for the more realistic three design/energy scenario pairs are reported. Specifically low density - business as usual energy strategies (LU1-Good), medium density - reasonable energy strategies (LU2-Better) and high density - aspirational energy strategies 

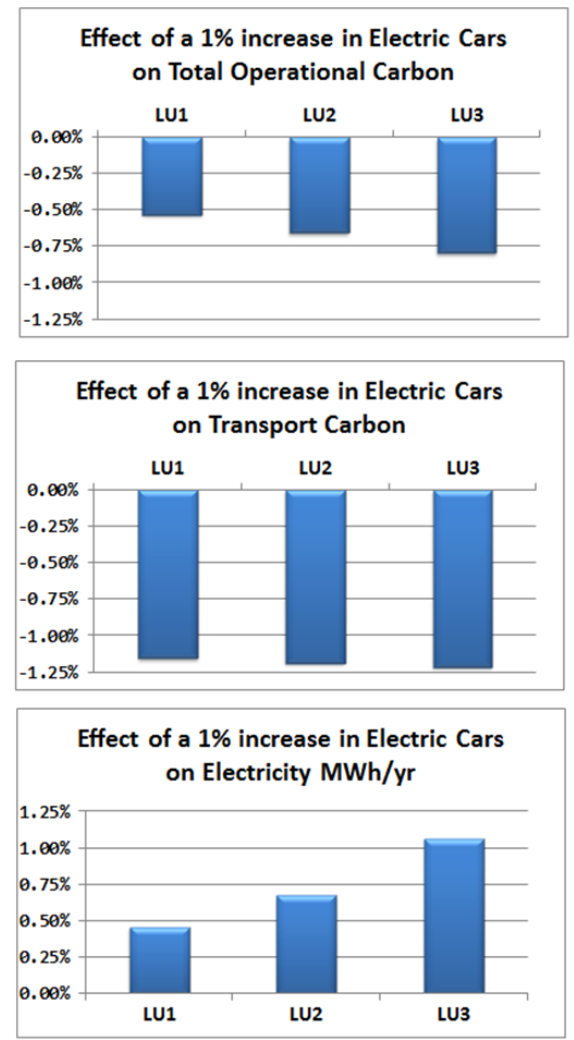

\section{Effect of Electric Cars upon Operational Carbon, Transport Carbon \& Electricity consumption}

Carbon Emissions per annum

Good Better

Best

Energy Strategies Energy Strategies Energy Strategies

Design 1

Low Density

(1x)
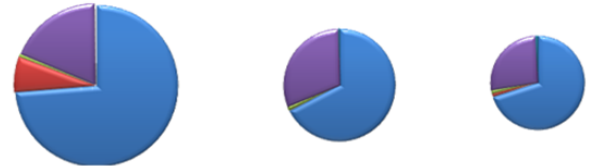

Design 2

Med Density

(4x)
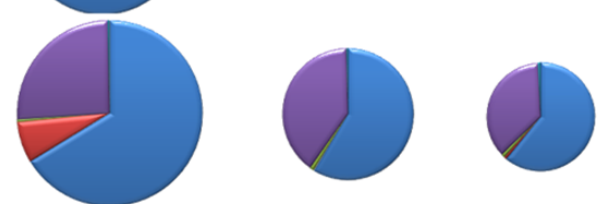

Design 3

High Density

$(8 \mathrm{x})$
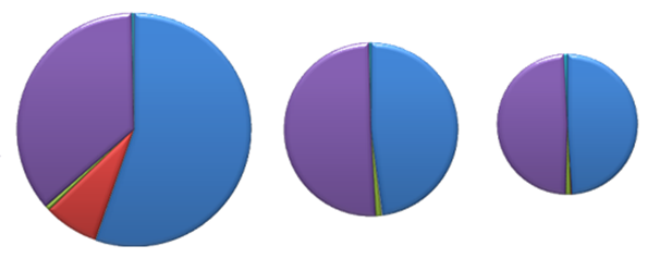

$\square$ Electicity $\square$ Thermal $\square$ Transport $\square$ Waste $\square$ Water_Elec

Figure 4.18: Effect of Electric cars on operational and transport carbon emissions and on electricity consumption. Figures shown correspond to LU1-Good, LU2Better and LU3-Best. Note as the design becomes increasingly dense and carbon efficient the need to tackle the transport component of carbon is increasingly clear (see pie charts). 
(LU3-Best).

As one would expect the results show decreases in transport carbon and increases in electricity consumption as electric car ownership increases. These effects can be quantified and importantly show that overall operation carbon emissions decrease with the use of electric vehicles. Particularly for each $1 \%$ additional parking spaces filled by electric cars we see $1.1-1.25 \%$ decrease in transport carbon emissions and a corresponding $0.45-1.1 \%$ increase in electricity consumption resulting in an overall $0.55-0.80 \%$ decrease in operational carbon depending on design scenario thus validating that electric cars will indeed have a positive effect on the development.

The interesting insight in these results is the increasing percentage "return" for each increase in electric car ownership. For example consider the operational carbon graph in Fig. 4.18 (p174) where a 1\% increase in electric car ownership results in a 50\% larger decrease in operational carbon under the most dense scenario compared to the least dense. However given that the decrease in transportation related carbon emissions is pretty much constant across the scenarios something else must be responsible for this trend.

The pie charts shown in Fig. 4.18 (p174) identifies what is going on by showing the total operational carbon emissions per annum broken down by source. We see that as the density and population of the masterplan increase the total emissions also increase, however this trend is counteracted by the use of increasingly expensive energy strategies. Note that the bar charts correspond to the top-left to bottom-right diagonal line of pie charts. From these we see that the proportion of total operation emissions from transportation increases steadily from LU1-Good to LU3-Best. This increasing proportion is due both to an increasing population using more transportation and an increasingly effective set of energy strategies reducing other sources of operational carbon. This trend explains why a unit decrease in carbon emissions would have a large percentage impact across, as is shown in the operational carbon bar chart. A similar story explains the trend in electricity consumption.

The insight from these trends is that as the development becomes more dense and a larger set of energy strategies are employed it becomes more and more critical to impact the transportation related carbon emissions and the introduction of electric cars provides an increasingly effective mechanism for doing this, provided of course the increase in electricity consumption can be absorbed in a carbon efficient way.

\title{
4.12.2 Optimal Density for District Energy System
}

\author{
What is the optimal (or critical) density for the district energy \\ system?
}


District Energy Systems provide a more efficient and potentially cheaper means of powering, heating and cooling a residential or commercial district. Such systems work by networking together the power systems for a group of buildings across a compact area using a system of steam or hot or chilled water pipes. This allows centralisation of the heating or cooling infrastructure enabling larger more efficient appliances to be used. Such appliances may then provide other benefits such as tri-generation plants which produce electricity from excess heat. The networking of buildings energy demands enables smoothing of loads between buildings resulting in more efficient operation. Some district energy systems also allow piping of excess heat / cooling between buildings. For example using excess data centre heat to heat offices or houses, or using the heat from offices to heat homes during the evening. Thus from a carbon efficiency perspective district energy systems can be very appealing.

However there is a requirement that sufficient demand and supply can be coalesced in a sufficiently geographically local area in order for a district energy system to be efficient. This is due to thermal lost/gain from longer pipes and pumping energy over a greater distance compounded by a greater construction and operating cost.

For the CommunityB project we used Arup's District Energy Feasibility (DEF) model described in section 4.4.3 (p145) to compare options for the adoption of a district energy system as the model enables comparison of several of forms of district energy system.

To investigate this question it was decided to explore the design space by changing the density of the development according to the Floor Area Ratio (FAR) metric. FAR is a measure of density defined by

$$
\frac{\text { Gross Floor Area (GFA) }}{\text { Site Area }}
$$

To explore a range of FAR values we varied the site area whilst keeping the GFA of the development constant. Conceptually this has the effect of compacting the development into a smaller area thus creating higher buildings. A typical range of FAR values for the area of CommunityB would be 0.5 to 3.0 , from these a set of landuse programs were developed and analysed using HierSynth.

Results of this investigation are shown in Fig. 4.19 (p177) which uses a composite energy consumption and carbon emissions costing metric developed by the analysis team. Three trends are shown for the three different energy scenarios under consideration. For each of these we see that there is a exponential decay in the cost metric as density increases with FAR 1.0 being half that of FAR 0.5. After FAR 1.0 we see most of the benefit of increasing FAR is achieved by FAR 1.5. Finally we see more evidence for the adoption of better energy strategies with the "Better" and "Best" strategies resulting in half the cost of the business as usual "Good" strategies. 


\section{Ideal Density for District Energy System}

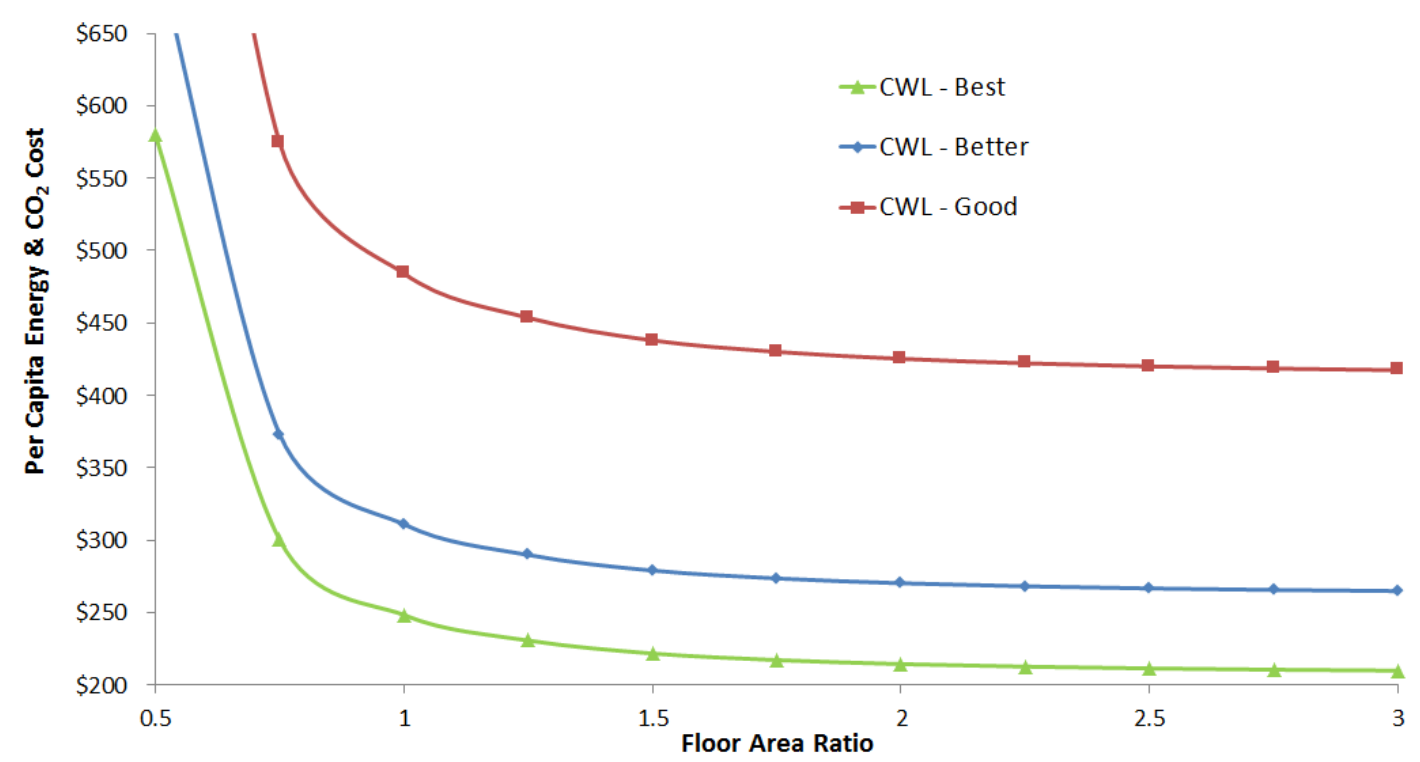

Figure 4.19: Composite energy and carbon costing metric identifies the effect of varying the density of a design under three different energy scenarios. The majority of the benefit of increasing density is achieved by FAR 1.5.

This investigation coupled with the results discussed previously in the chapter gave additional evidence to the team to influence the design toward a more dense development.

\subsubsection{Residential and Commercial Mix}

\section{What is the optimal mix of residential and commercial develop- ment for heat recovery and reduced vehicle miles travelled?}

This question concerns the landuse mix of the development and its impact upon the performance of a district energy system and transportation carbon emissions. This is an interesting questions as there are a number of expected phenomena which are likely to be at work.

For district energy systems one would expect a more equal mix of residential and commercial development to enable the balancing of heating and cooling loads between housing and offices to create a smoother more efficient energy demand. Similarly the different demand profiles through the day from each source may enable possibility of piping heat between the different land use types.

The expected effect on transport would be for an optimal reduction in transportation carbon emissions with a good mix of commercial to residential land use as more of the office workers are able to live and work in the same area and so use low carbon 
transportation such as taking the bus, biking or walking to work.

To investigate these expectations we used HierSynth and a set of designs created by modifying a base design according to the following algorithm. To change the residential and commercial mix we found the total GFA of residential and office development in the base design. We then distributed this total between office and residential development starting with $0 \%$ residential and moving to $100 \%$ in $10 \%$ increments. We note that office GFA is split between office types in same proportion as in base design.

This set of scenarios were then analysed using the integrated analysis described in section 4.9 (p157). The results of this investigation can be split into three parts: firstly the impact upon a district energy system; secondly the impact upon transportation and finally we looked at adding extra residential GFA to the design rather than converting office development to residential.

\section{Optimal District Energy System}

This section looks at the impact of different mixes of residential / commercial development upon a district energy system. Results in this section are reported percapita as the number of residents and office works will vary between scenarios. These results are produced from identical analysis runs differing only by the inclusion or not of a district energy system and hence will be independent of effects from transportation which are discussed in the next section on page 181 .

Fig. 4.20 (p179) shows the percapita carbon savings from adopting a district energy system in the development over the range of residential / commercial mixes under the "Good", "Better" and "Best" energy scenarios. Firstly we see that using a district energy system produces carbon savings of up to $30 \%$. As expected the graph is " $\cap$ " shaped with the optimal development mixes at $40 \ldots 60 \%$; depending on energy scenario with the most effective energy scenario ("Best") peaking at a lower residential mix than the business as ususal case ("Good"). This is probably to be due to the point at which the energy demand from residential and commerical landuse balence. Since under the less effective "Good" energy strategy there will be a larger energy demand from commerical development than under the "Better" or "Best" energy scenarios it will take a correspondingly larger amount of residential development to provide balance. The effectiveness of the different energy scenarios can be seen in Fig. 4.4 (p152).

Surprisingly the trends are very flat between $0 \% \ldots 60 \%$ this is likely due to excess system capacity slowly absorbing the residential demand. Similarly the sharp drop after $70 \%$ residential is likely to be the point at which the capacity of the system is exceeded and other technologies are required to meet peak demand.

This is confirmed by considering the annual percapita gas consumption as shown in Fig.4.21. Here we see that the system capacity absorbs any increase in thermal demand up until the same points of inflection in Fig.4.20 after which gas consumption 


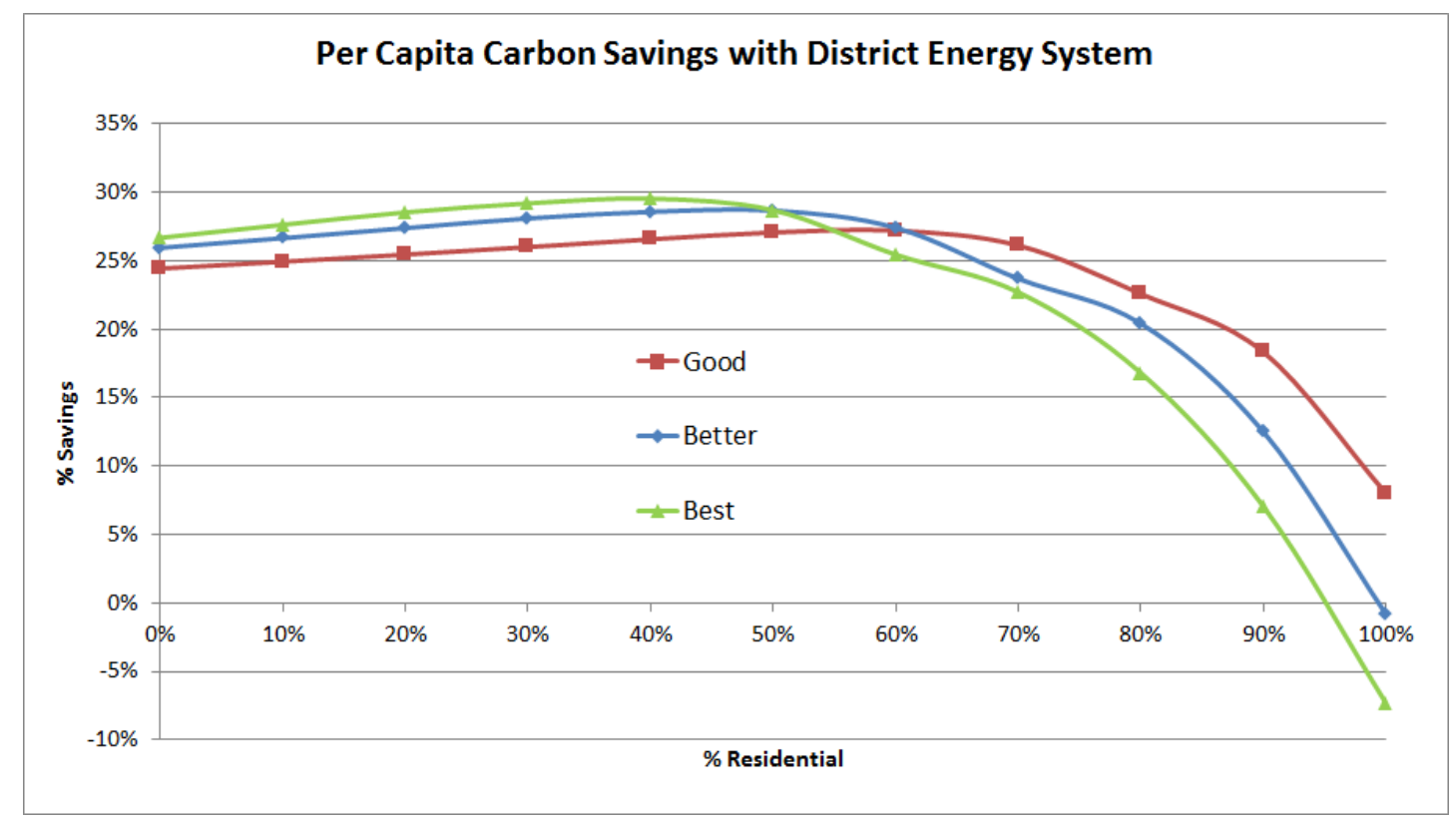

Figure 4.20: Percapita carbon savings with district energy system as the residential proportion of the development is increased. Notice different maxima under different energy scenarios. Expectation was for a strongly " $\cap$ " shaped graph as a balance between building types enables lower peak demand and a more smooth energy demand. Fig 4.21 (p180) identifies part of the reason that this expected trend was not seen. 


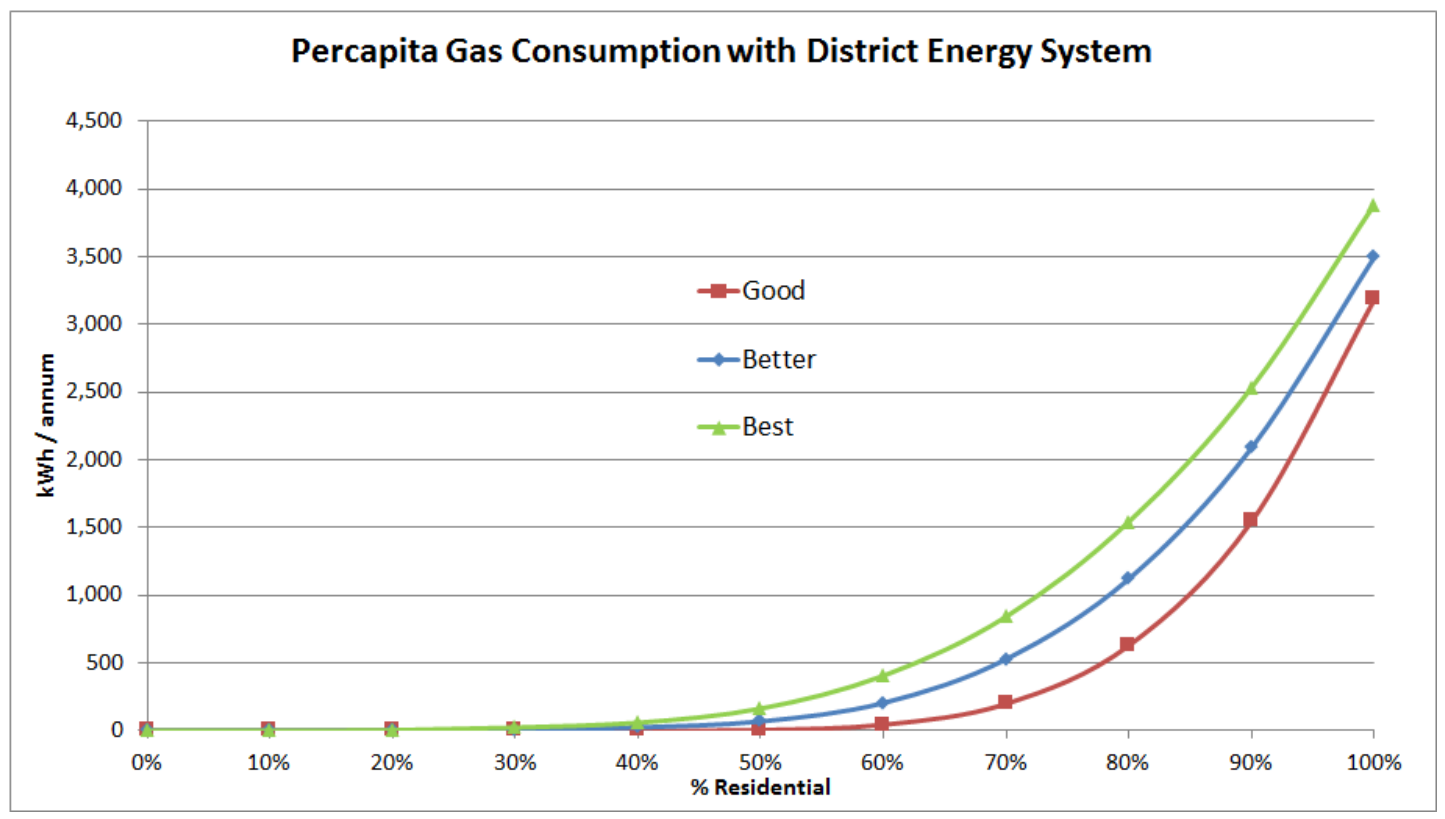

Figure 4.21: Percapita gas consumption with a district energy system as the residential proportion of the development is increased. Notice how the capacity of the system negates the need for gas consumption in the development up to the point where additional technologies are required.

rises dramatically as other technologies are required to meet the peak demand from housing. This exponential rise accounts for the decline in carbon efficiency seen in the previous figure.

Finally we can also consider the impact upon electricity consumption as shown in Fig. 4.22 (p181) which shows a similar trend with decreasing electricity savings up to the same points of inflection as identified previously. One unexpected feature of this graph is that the "Good" energy strategy results in higher savings than the "Best" strategy, this is likely because as more energy strategies are enabled under "Best" more of the potential savings from a district energy system have already been made by other strategies.

In conclusion we see that to minimise operational carbon a residential mix of between $40-60 \%$ of the total GFA is ideal depending upon the energy strategies employed in the development. However given the shallowness of the carbon curve anything up to $60 \%$ residential would produce a good carbon reduction. Certainly with a view to avoiding gas consumption a residential mix of $30 \%$ or less would be ideal, which will also produce a decrease in electrical consumption.

Hence from an energy perspective a residential mix of upto $30 \%$ is ideal, with more residents reducing percapita carbon although percapita gas and electricity consumption begining to rise after $30 \%$. 


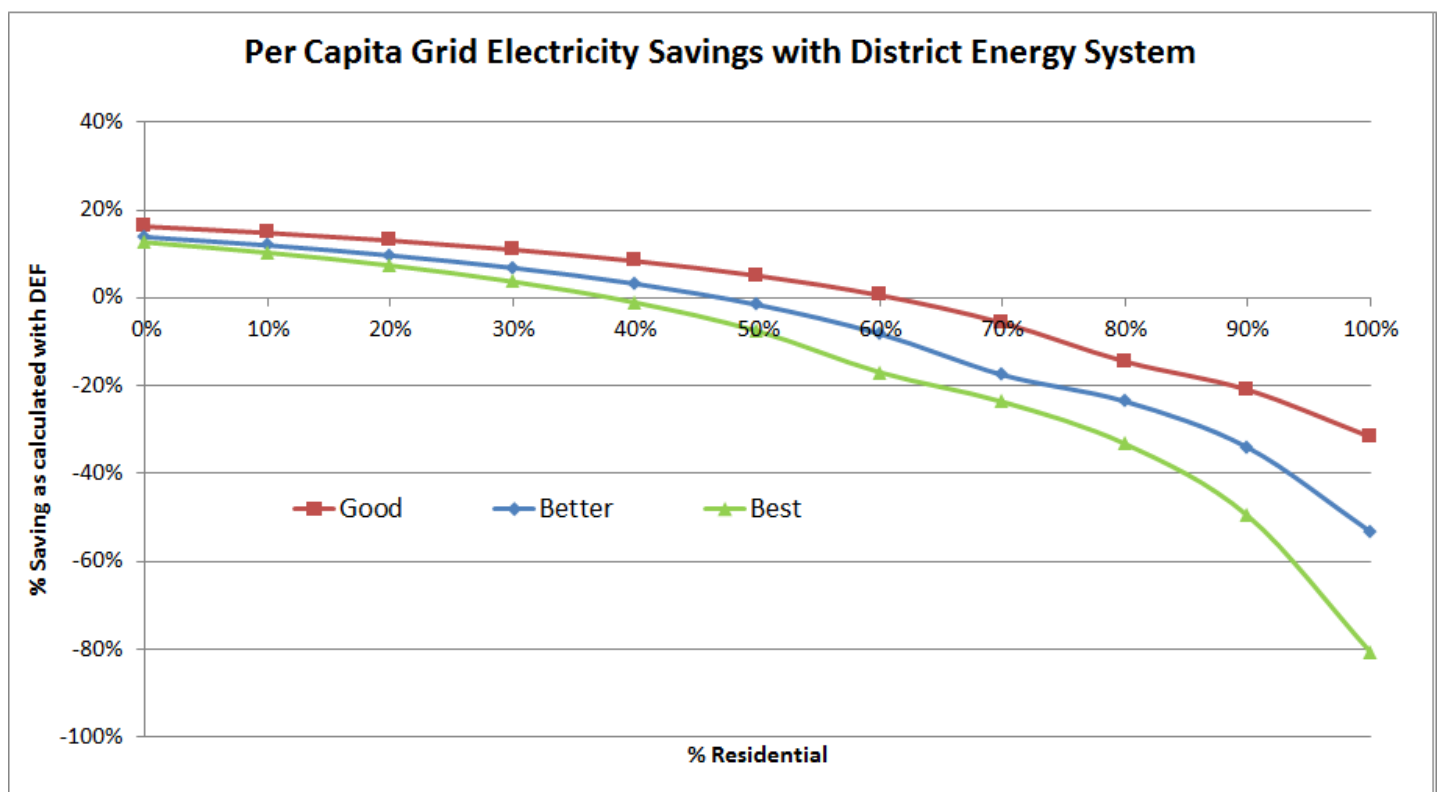

Figure 4.22: Percapita electricity saving with a district energy system as the residential proportion of the development is increased. Note that the intersection points with the zero line, match the points of inflection shown in Figs. $4.20(\mathrm{p} 179)$ and $4.21(\mathrm{p} 180)$.

\section{Transport Carbon}

We now look at the impact of different mixes of residential / commercial development upon transportation, specifically on percapita carbon emissions from transport. The expectation of this part of the investigation is that a good mix of residential and commercial development would enable people to live near where they work and so be able to walk, cycle or get the bus to work, each of which is a less polluting form of transport than commuting by car. This should result in a roughly " $\cup$ " curve as the residential proportion increases from $0 \%$ to $100 \%$.

To answer this question we used the same set of designs with varying residential / commercial mix from the previous section and augmented them with different assumptions about the modal split of the populations transportation choices. These modal splits were developed by Arup's transportation team and are shown in Fig. 4.23 (p182).

From these modal splits we see the expected shift in modes of transport away from the heavily polluting car to other modes of transport (notably the local bus) with minimum car usage at $30 \%$ residential. This should result in the expected " $\bigcup$ " curve for carbon emissions.

Having used HierSynth to analyse these scenarios, Fig. 4.24 (p183) shows the annual percapita transport carbon emissions. Surprisingly we see a " $"$ trend with local minimum at $30 \%$ and $100 \%$ instead of the expected " $\cup$ " graph. Particularly suprising 


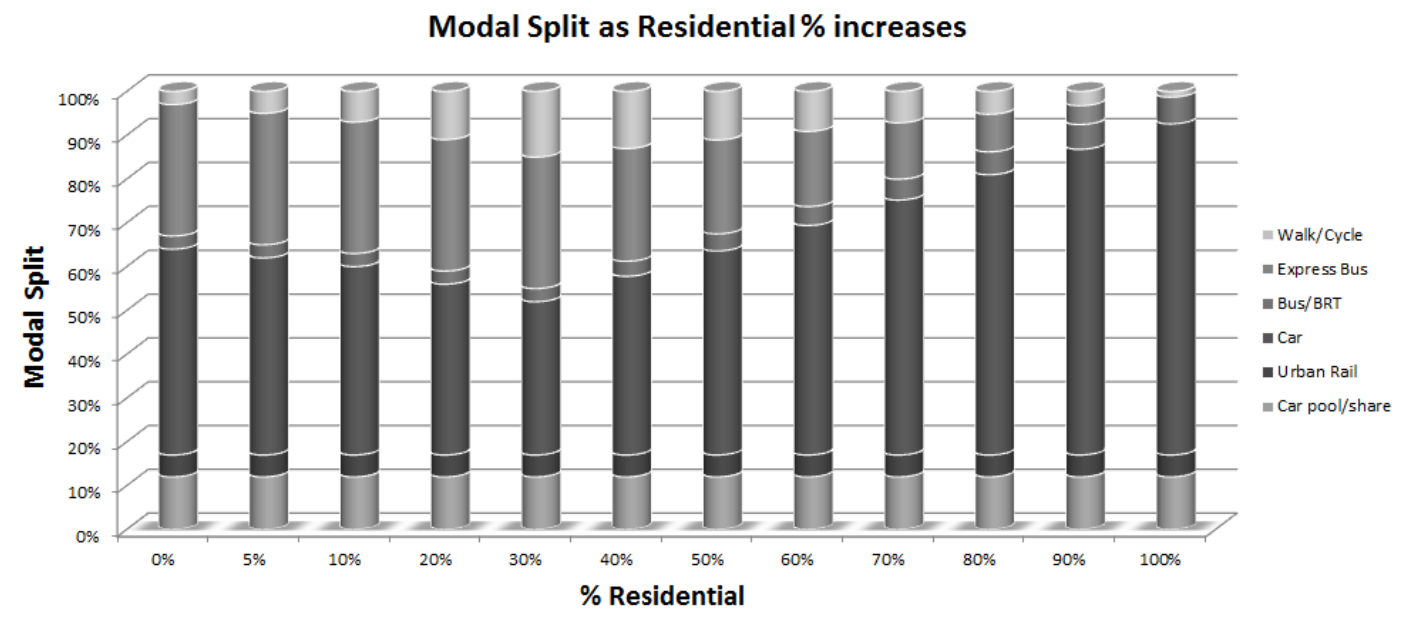

Figure 4.23: Transportation modal splits as residential as percentage of development that is residential increases. Note " $\cup$ " shape in the most heavily polluting forms of transport (cars).

was the downward trend from $60 \%$ which is the opposite of that expected.

The graphs in Fig. 4.25 (p184) explore the reasons for this unexpected trend. All of these graphs were generated using HierSynth to extract data from the IRM model.

Graph $a$ ) shows that as we move $M^{2}$ of office into $M^{2}$ of residential (keeping the same total $M^{2}$ and density of the original design) the population falls since offices have more workers per $M^{2}$ than houses have residents per $M^{2}$. With an average resident tending to travel less than an average commuter this would begin to account for the downward trend on highly residential developments. This is shown by graph $b$ ) showing the number of kilometers travelled per day though we note that this trend is compounded by the larger number of workers compared to residents.

If we break this figure down by mode of transport and by residents (graph $c$ ) and workers (graph $d$ ) we see the domination of buses and cars and again see how workers travel far further than residents. Note the " $\backsim$ " trend in car travel for workers.

If we then convert vehicle kilometres into carbon emissions in graphs $e$ ) and $f$ ) we see that results are dominated by petrol car emissions. We also see in graph $e$ ) the upward trend we were expecting beyond $50 \%$ however it is not sufficiently large to counteract the downward trend in graph $f$ ).

To summarise, there is no " $\cup$ " shape trend because:

- Transport carbon emissions are dominated by cars. Whilst residential carbon emissions from cars increases with residential \% (graph $e$ ), it does not rise sharply enough to counteract the fall in car pollution from office workers not commuting into the area (graph $f)$.

- The average resident does not travel as much as the average commuter (graph $b$ ). 


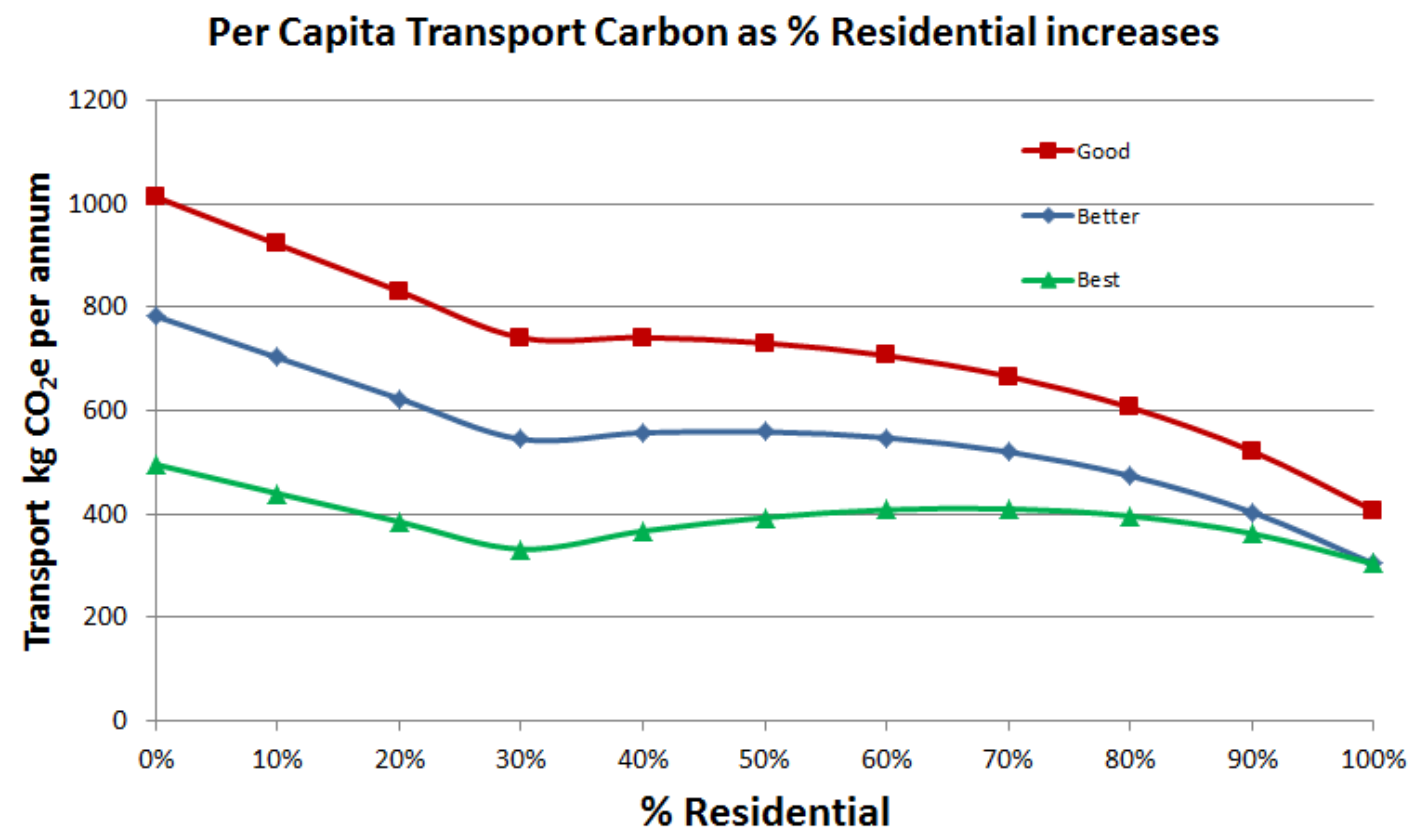

Figure 4.24: Percapita transport carbon varying with the residential fraction of the development. Unexpectedly we see an " $\backsim$ " shape rather than the expected " $\cup "$ shape. Reasons for this are discussed in section 4.12 .3 (p181)

- There are fewer residents then workers for the same $M^{2}$ (graph $a$ ).

This result was highly unexpected by the analysis team. Also surprising in Fig. 4.24 (p183) is that under the "Best" energy scenario a 70\% commercial development can have identical percapita transportation carbon emissions as a fully residential one. This highlights independently from the previous section on energy consumption that having $30 \%$ residential development would greatly improve the carbon profile of the development.

\section{Adding Residents}

Finally for CommunityB a more likely scenario than replacing offices GFA with residential is to simply add residential GFA to the development since this keeps the same amount of office space. This would have a side effect of increasing the density of the development which as we saw in section 4.12 .2 (p175) will improve the sustainability of the development.

To investigate this scenario we set up a number of designs adding from $0 \ldots 100 \%$ more GFA to the development as housing. These designs were then analysed using HierSynth.

The results of these scenarios are mapped in Fig. 4.26 (p185) this time normalised by $M^{2}$. We see that in confirmation of investigations into increasing density (section 
a) Development Population

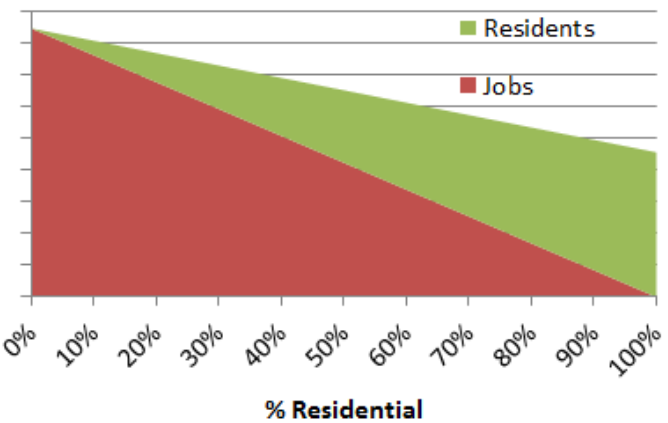

c) Resident Vehicle km travelled per day by mode

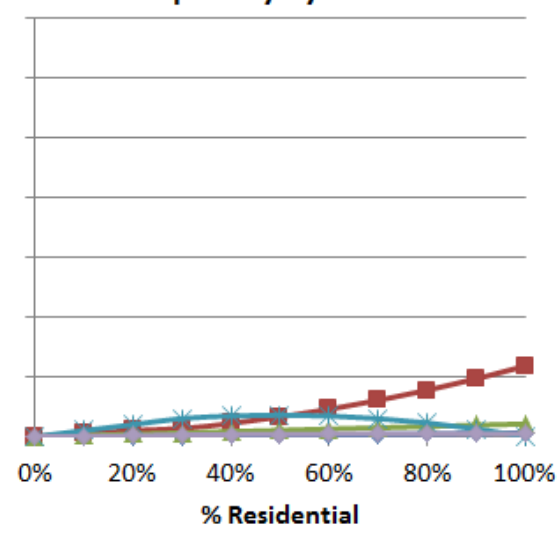

e) Resident Transport Carbon Emissions

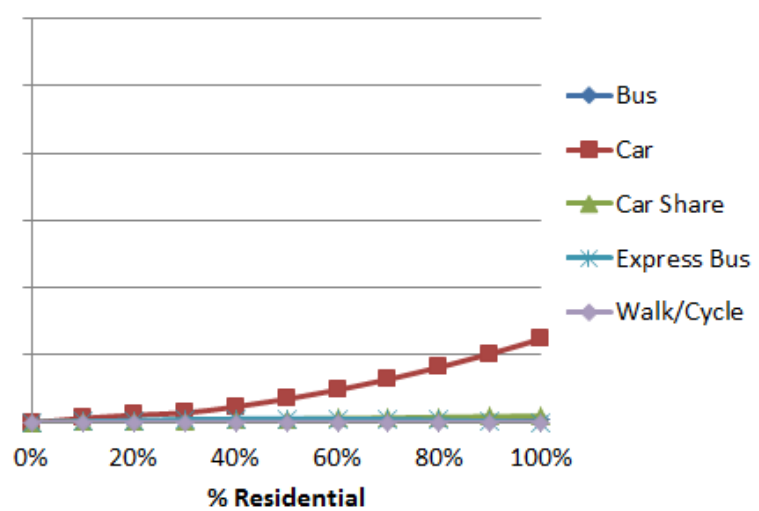

b) Vehicle $\mathrm{km}$ traveled per day

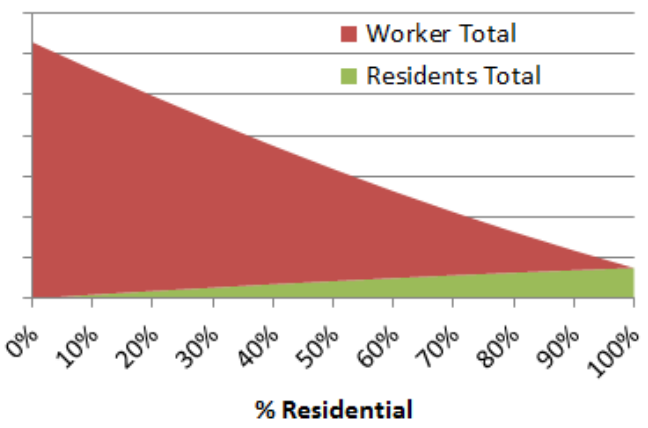

d) Worker Vehicle km travelled per day by mode

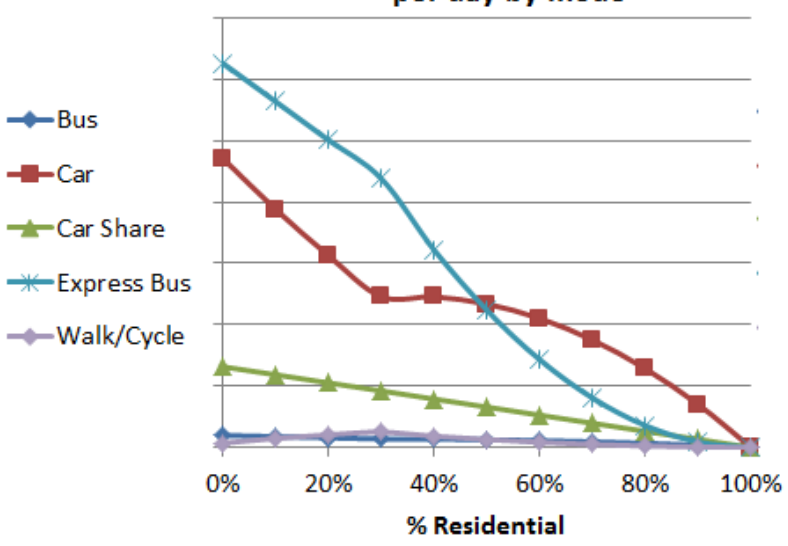

f) Worker Transport Carbon Emissions

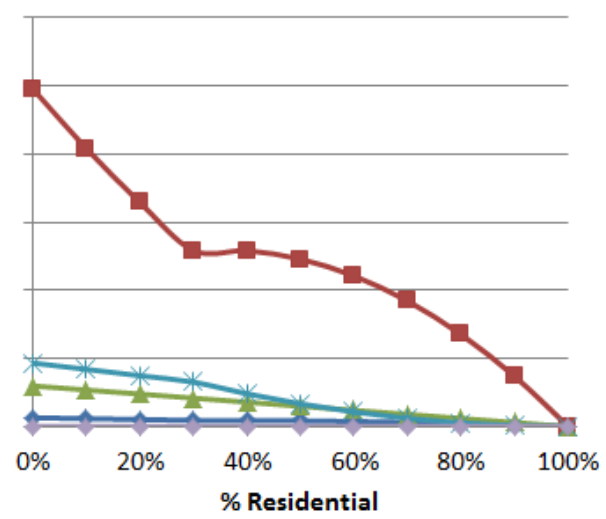

Figure 4.25: These graphs explore the trends identified in Fig.4.24 of the impact of residential / commercial mix on transportation carbon emissions. Note that scales have been removed to preserve commercial confidentiality. Graphs c \& d share the same scale. Graphs e \& f also share a scale. 


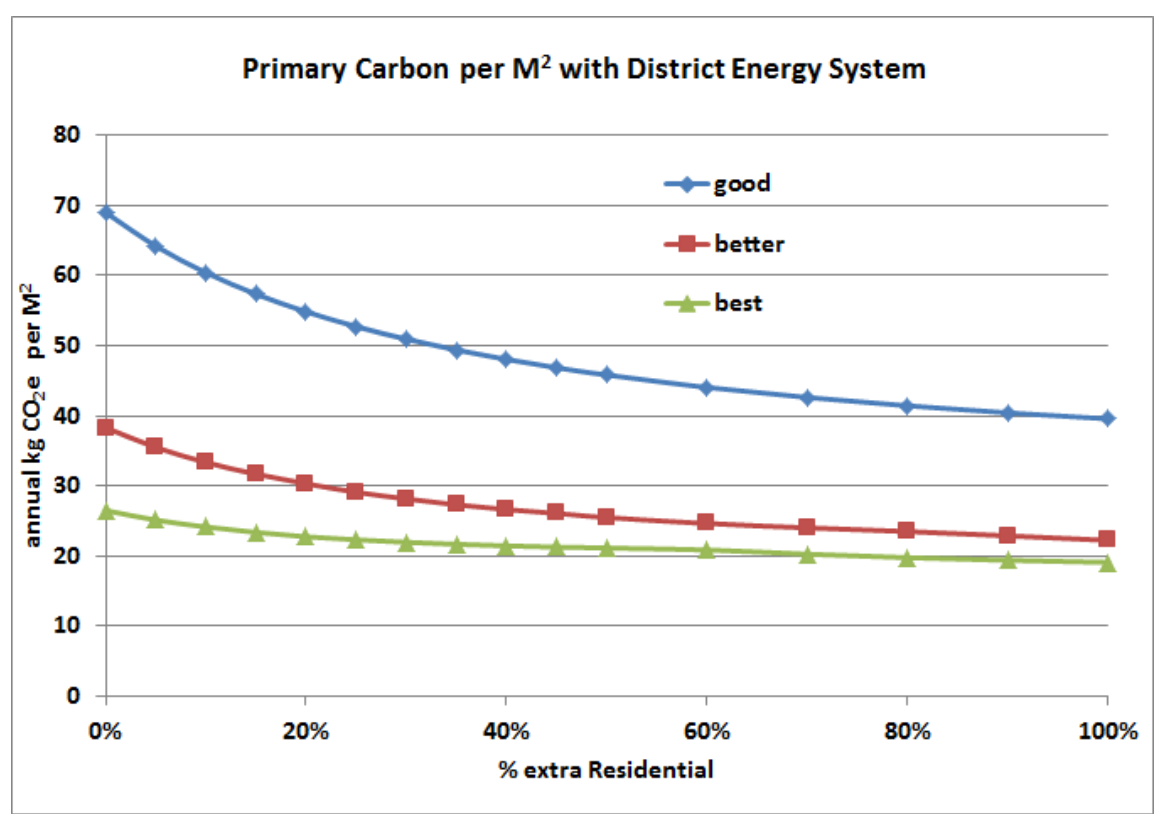

Figure 4.26: Effect of adding residential GFA into the development upon operational carbon per annum. Note that most of the gain from adding residents is acheived by adding $35 \%$ extra residential GFA corresponding to the $30 / 70 \%$ residential/commercial sweet point identified in section 4.12 .3 (p181).

4.12.2 (p175)) increasing the residential GFA of the development results in carbon reductions. We see that half of the effect at 100\% extra GFA as residential is had at just $20 \%$ extra residential. Reasons for this trend include the increase in density, the increasing efficiency of the district energy system employed as the residential to commercial balence changes and decreases in transport carbon identified in the previous section as well as changes in the average energy use intensity of an average $M^{2}$ in the development. We note that most of the gain from adding residents is acheived by adding $35 \%$ extra residential GFA corresponding to the $30 / 70 \%$ residential/commercial optimal point identified in section 4.12 .3 (p181).

It is also interesting to look at the effect of these scenarios on the feasibilty of adding a district energy system to the development. Fig. 4.27 (p186) shows this impact and is analogous to the first half of Fig. 4.20 (p179). Again we see that with more efficient office space producing less energy demand under the "Better" and "Best" energy scenarios it requires less residential development to provide an optimal balance than under the business as usual "Good" case as shown by the earlier graph maxima. Finally we see that for the "Better" and "Best" energy scenarios adding $25-35 \%$ extra residential provides an optimal district energy system. At $35 \%$ this corresponds approximately to a $30 / 70 \%$ residential to commercial development. Suggesting that the same benefits identified earlier from a $30 \%$ residential development can be had by 


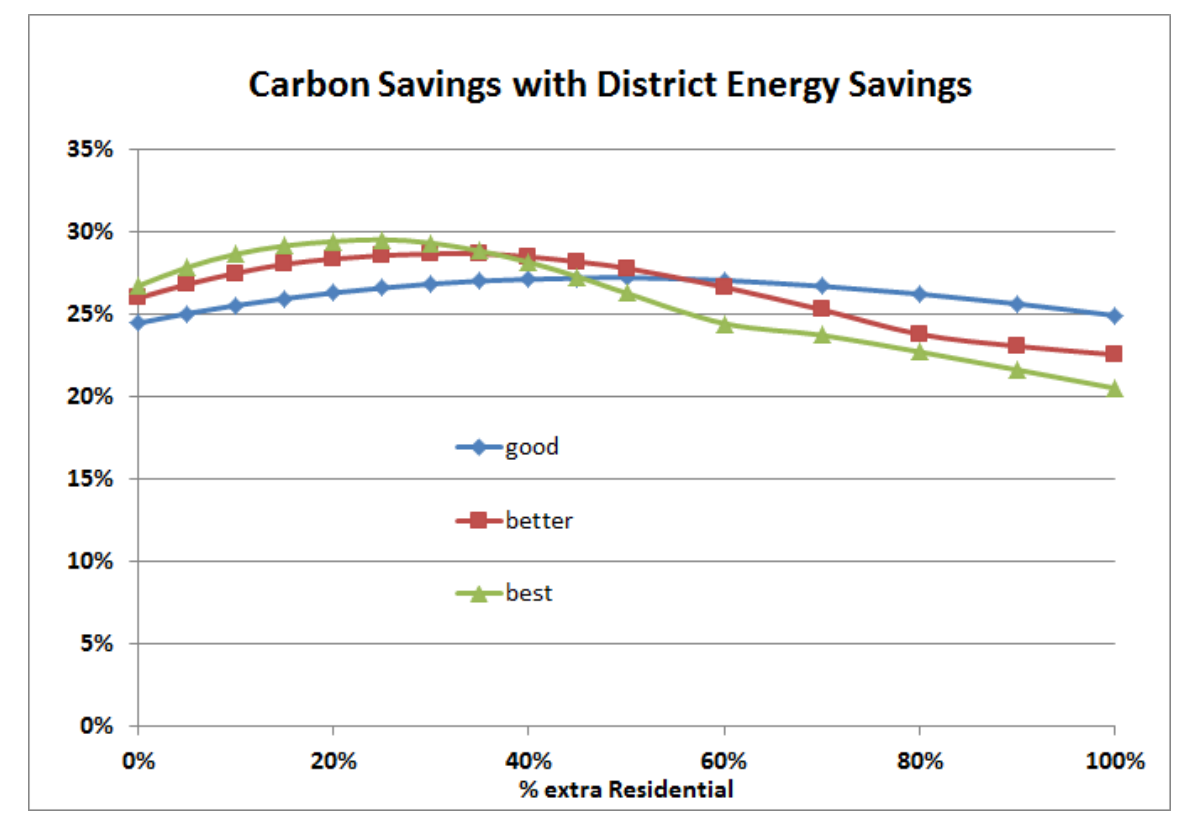

Figure 4.27: Effect of adding residential GFA into the development upon carbon savings achieved by adding a district energy system. Trends should be compared to the first half of Fig. 4.20 (p179).

adding more residential GFA rather than by replacing office GFA.

\section{Conclusions}

\section{What is the optimal mix of residential and commercial develop- ment for heat recovery and reduced vehicle miles travelled?}

From these investigations carried out using HierSynth we can firmly conclude that from the perspectives of carbon emissions, transport carbon, gas consumption and an optimal district energy system efficiency a mix of $30 \%$ residential, $70 \%$ commercial development is critical. We have also identified that the same effect can be achevied by adding residential GFA to the development instead of reducing commercial development. This would have the effect of increasing the density of the development which as we have shown also has benefits for the sustainability of CommunityB . Hence a good case can be made for increasing the density of the development by increasing the residential GFA something which should appeal to all stakeholders in the project. Such investigations would have proved prohibitively expensive without the use of a computational framework such as HierSynth. 


\subsection{Sensitivity Analysis}

In this section we make use of the sensitivity analysis tools integrated into the HierSynth framework to investigate Arup's IRM model as applied to the CommunityB development. We seek first to identify the best means to impact a number of KPIs based upon the 1,224 inputs and assumptions identified in the model with the spreadsheet analysis tools discussed in the next chapter. Secondly we look at the impact of the energy strategies embedded in the IRM model to help the design of different energy scenarios. These gave many insights into both the design, the design space and the IRM model itself.

\subsubsection{IRM analysis}

\section{"What are the development's carbon emissions most sensitive to? Which of these sensitivities can we positively impact?"}

We use a Plackett-Burman (PB) sensitivity analysis [81] [106] to investigate the IRM model and the scope for impacting a set of identified KPIs which the model calculates. For each input factor investigated, a high and a low level is required to be set. A number of scenarios are then generated, run and then analysed using the PB analysis. A PB analysis will identify the factors (inputs) that give the most scope for affecting a given KPI. For example, the 12 most effective means of reducing percapita carbon emissions can be identified from hundreds of inputs. More information on the techniques employed can be found in section 5.11 (p229).

For this analysis we used the 1,224 inputs to Arup's IRM model identified in Fig. 4.2 (p147). Each of these was given a meaningful name and a suitable high and low value was set in collaboration with the analysis team. Note that not all of these inputs were actually variable (e.g. conversion factors), those that did vary were either design variables set by the design team (e.g. land use schedule) or alternatively they were analysis assumptions such as the carbon emissions coefficient for the national grid. Design variables should be set to show the maximum scope for design variation and mark the "limits" of the design space. For analysis assumptions high/low values were used to bracket uncertainty in the model. Since considering multiple KPIs is computationally cheap we considered the sensitivity of the full set of 200+ IRM KPIs used throughout this collaboration.

Whilst it is possible to use a HierSynth execution chain to run a sensitivity analysis (as is shown in Fig. 3.18 (p130) and described in section 3.14 (p127)) the number of analysis factors involved meant that a simpler one tree with one analysis model was more computationally tractable and it is the results of this that are reported.

As an example of the insight generated through the sensitivity analysis performed using HierSynth we report the top twelve inputs with most scope to impact percapita 


\begin{tabular}{|c|c|}
\hline \multicolumn{2}{|c|}{ Normalised Sensitivity } \\
\hline Variable & Primary Carbon percapita $\mathrm{V}$ \\
\hline Gasoline Car Carbon Emissions & 100 \\
\hline Electricity Grid Carbon Emissions & 789 \\
\hline Passenger Kms New Office & 84 \\
\hline Gas Carbon Emissions & 25 \\
\hline New Office - Appliances Energy & 20 \\
\hline Building Efficiency District 2 & 16 \\
\hline Old Office - Appliances Energy & 16 \\
\hline Building Efficiency District 6 & 12 \\
\hline Building Efficiency District 3 & 11 \\
\hline Passenger Kms Old Office & 11 \\
\hline Shuttle Bus Usage & 11 \\
\hline Percentage waste Landfilled & $\square$ \\
\hline
\end{tabular}

Figure 4.28: Sensitivity analysis results for the operational carbon percapita KPI. We normalise results to the most impactful variable. Over 1,200 variables were analysed as part of this analysis.

carbon emissions and annual waste water generation. These are two KPIs of around 200 for which a sensitivity analysis was performed.

Fig. 4.28 (p188) shows the scope for impacting percapita operational carbon. Here we see that variation in the carbon emissions of petrol cars has greatest scope for impacting percapita carbon emissions. In practice, this might result in a low emissions zone around the development or inducements and provision for electric cars (particularly given the benefits identified in section 4.12 .1 (p172)). The remaining sensitivities are normalised to this factor such that affecting the carbon emissions of the gas network has a quarter of the potential impact as petrol carbon emissions.

In these results a number of factors had unexpectedly higher scope for impact than was expected by the IRM engineer these included "thermal (gas) carbon emission factor", "landscape (site) carbon factor", the "buildings efficiency ratings" and the "shuttle/commuter bus carbon factor" factors. The reasons for these factors appearing unexpectedly high could be due to the high level of interconnectedness in the model meaning there are more real life relationships are modelled which can be difficult to predict. Alternatively, results may be inaccurate due to the high/low range for a factor being set disproportionately wide compared to other factors giving disproportionately higher scope for impact. Finally this maybe due to bugs in the model. Indeed close examination of the sensitivity analysis results did result in bugs being identified:

"This led me to realize I missed a needed correction in the Carbon tab. The carbon electricity factor for all the buildings that reference the site car- 


\begin{tabular}{|c|c|}
\hline \multicolumn{2}{|c|}{ Normalised Sensitivity } \\
\hline Variable & Waste Water per Annum \\
\hline Water Consumption Housing & 100 \\
\hline Water Consumption Wellness & 92 \\
\hline Housing \% Water for Flushing & 73 \\
\hline Fitness \% Water for Flushing & 67 \\
\hline Water Consumption New Office & 35 \\
\hline Water Consumption Old Office & 31 \\
\hline New Office $\%$ Water for Flushing & 26 \\
\hline \# Building units District 2 & 25 \\
\hline \# Building units District 7 & 25 \\
\hline \# Housing units District 2 & 25 \\
\hline \# Housing units District 7 & 25 \\
\hline Old Office \% Water for Flushing & 23 \\
\hline
\end{tabular}

Figure 4.29: Sensitivity analysis results for the waste water per annum KPI. We normalise results to the most impactful variable. It is also possible to consider side effects upon other KPIs; over 200 of which were considered.

bon factor was overwritten for the last project. When reverting references back to the original ones, I missed this one. This is why the electricity factor was lower than thermal in all the building types." Design Team Member, Arup

These sensitivity analysis results will of course vary depending upon the design and energy scenario under consideration and comparisons between the sensitivities under different scenarios could prove interesting.

As a further example of the insight that can be generated from a sensitivity analysis we consider a sensitivity analysis was waste water production per annum in Fig. 4.29 (p189). From these top twelve factors we can identify that:

1. Despite representing a low percentage of the development GFA impacting the water consumption for housing gives the biggest scope for reducing waste water production.

2. Similarly fitness centers have a disproportionate scope for impact although this may not be unexpected since they will include swimming centers.

3. The potential impact to be had by changing the percentage of water flushed away is one of the biggest opportunities for impacting waste water production across all land use types and gives substantial support to the introduction and specification that the development must use low flush fixtures and fittings as far as possible. 
4. Despite the relatively low percentage of the development that will remain as existing (old) offices there is still nearly as much benefit to be had by impacting waste water production as with new build development. This is likely to be because new build will already have stringent water consumption limits built in and so there will be less scope for impact. This gives much support for the retrofitting of existing offices with low flush appliances.

To conclude, the sensitivity analysis of the IRM model gave useful insight into the design, the design space and the model being used for analysis. One final piece of insight from both sets of results presented here is as follows:

"This was all very valuable to knowing what makes the most difference, and made sense. It is also very informative which districts have the most influence, because they are not in order of GSF, nor office-only GSF, nor site acreage!" IRM Modeller

\subsubsection{Strategy Analysis}

\section{"How significant are the various (energy) strategies in reducing the developments carbon emissions and water intensity?"}

As we have seen in this chapter the set of energy strategies to be applied to the CommunityB development is one of the critical design decisions. Each of the "Good", "Better" and "Best" scenarios consists of a mix of up to sixty energy strategies all implemented to different degrees. Example strategies range from changing the modal split of transportation to the introduction of low flush fixtures and fittings to reduce water consumption. These 60 strategies cover a number of disciplines from energy demand reduction, water supply, waste reduction, transportation and waste diversion strategies. All of these strategies are simulated within Arup's IRM model and affect the highly interrelated multidisciplinary calculation of multiple KPIs across many disciplines.

Given this importance we decided to look into the consequences of each strategy under consideration. This was done by starting with an analysis run with all energy strategies turned off, then completing a series of analysis runs with each strategy turned on, one at a time, and measuring the impact upon a number of KPIs. KPIs considered include percapita carbon emissions, water consumption and electricity demand. This will show the benefits and drawbacks of each strategy and its side effects on other KPIs, with unexpected effects being of particular interest. However due to the type of sensitivity analysis employed this study omits interaction effects between strategies. This is due to the computational tractability of the analysis. 


\section{Implementation}

To enable greater insight to be generated this sensitivity analysis was under taken on the integrated model ensemble developed in section 4.9 (p157) and the effect of each strategy on 1,315 KPIs was measured across these 12 integrated analysis models. This should yield interesting results due to the interrelation of instances of Arup's IRM model with other analysis modules as shown in Fig. 4.9 (p160). Impacts in one model may result in corresponding effects in subsequent integrated models. Since each design will be impacted differently by each energy strategy we undertook to carry out this investigation on all 3 Land Use designs (LU1 - 3) under each of the "Good", "Better" and "Best" energy scenarios as described in section 4.6 (p148).

In practice, a HierSynth execution chain was used for this investigation and is shown in Fig. 4.30 (p192). First the execution chain is executed under the final construction phase scenario, although results could have been generated for each construction phase the final phase representing completed development of CommunityB was chosen for this study. This scenario was then used to execute the scenario generation tree discussed in section 4.9 (p157) which results in 9 design / energy strategy scenarios. Then a second scenario generation tree is used. This tree also used root first execution (see section 3.11 (p120)) which queries a large matrix for a particular column. This matrix has rows representing different energy strategies and columns representing different analysis runs. The matrix is essentially the identity matrix consisting of True and False values such that each energy strategy is turned on one at a time. An initial "all off" column is added as a control. Hence the query node produces 61 child nodes each of which creates a new scenario each time it is executed. Executed sequentially these two trees produce 549 analysis scenarios and for each of these scenarios the model ensemble created in section 4.9 (p157) is run and the energy strategies are turned on and off in all of the IRM models in the HierSynth tree according to the scenario. Across these 12 models and some 1,315 KPIs are read. HierSynth took around six hours to compute these 6, 588 analysis runs using four concurrent Excel instances.

\section{Strategy Effects}

Sample results from this analysis are shown in table 4.1 (p193) which demonstrates the measured impact of four strategies on one design under one energy scenario as measured by one instance of the IRM model. Results in this table are relative to no energy strategies being employed and show the changes identified in the KPIs computed by the IRM model. Several unexpected consequences of strategies were identified in this way, for example the energy saving from water efficiency was unexpected, however this makes sense since less water consumption requires less pumping, heating, treatment and sewerage energy related costs. For the same reason the black (waste) water treatment 


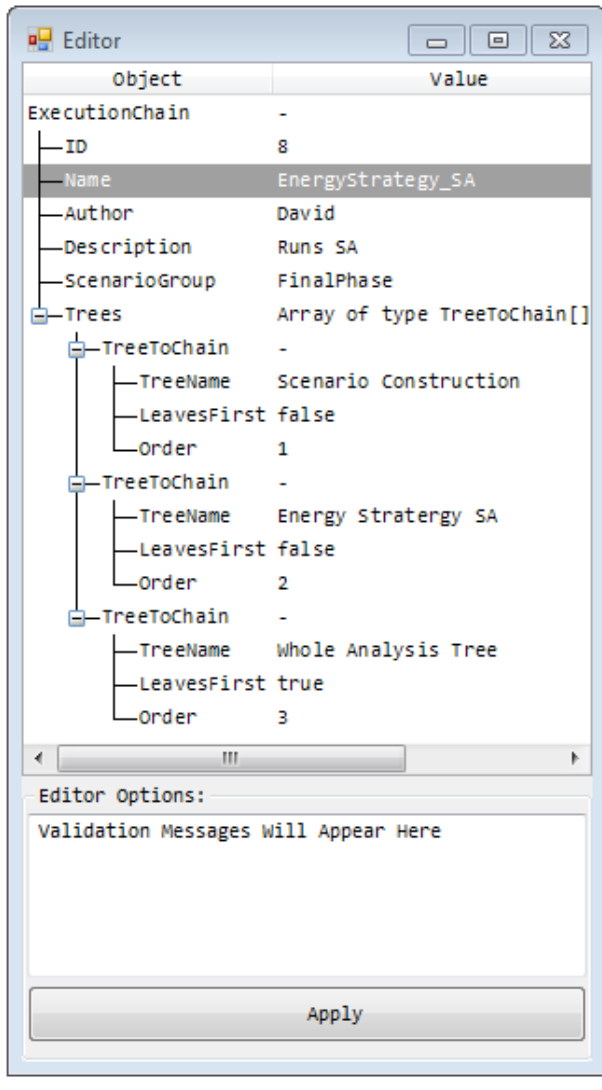

Editor Options:

Validation Messages Will Appear Here

Apply

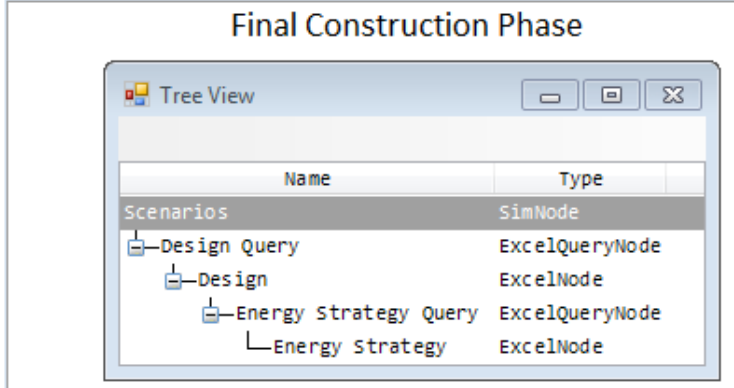

3 Designs * 3 Energy Strategies $=9$ Scenarios

\begin{tabular}{|c|c|}
\hline 喝 Tree View & \\
\hline \multicolumn{1}{|c|}{ Name } & \multicolumn{1}{c|}{ Type } \\
\hline Energy Strategies SA & Simvode \\
\hline $\begin{array}{c}\text { 1-SA Strategy Query } \\
\text { LStrategy SA }\end{array}$ & ExcelQueryNode \\
\hline
\end{tabular}

9 Scenarios * (Control +60 Strategies $)=549$ Scenarios

\begin{tabular}{|c|c|c|}
\hline 㟧 Tree View & $\square$ & 回 \\
\hline Name & \multicolumn{2}{|r|}{ Type } \\
\hline Development & \multicolumn{2}{|c|}{ SimNode } \\
\hline -Analysis_IRM_for_EUI & \multicolumn{2}{|c|}{ Excelnode } \\
\hline -Analysis_DEF & \multicolumn{2}{|c|}{ Excelnode } \\
\hline -Analysis_IRM_for_consumption_DWL & \multicolumn{2}{|c|}{ Excelnode } \\
\hline -Analysis_IRM_for_consumption_NO_DWL & \multicolumn{2}{|c|}{ Excelnode } \\
\hline -Analysis_LCCA & \multicolumn{2}{|c|}{ Excelnode } \\
\hline -Analysis_IRM_for_consumption_DWL_Supply & \multicolumn{2}{|c|}{ ExcelNode } \\
\hline 1-DEF_Building Type_Query & \multicolumn{2}{|c|}{ ExcelQueryNode } \\
\hline LPerBuild ingTypeAnalys is_DEF & \multicolumn{2}{|c|}{ ExcelNode } \\
\hline
\end{tabular}

549 Scenarios * 12 Analyses $=6,588$ Analyses 549 Scenarios * 1,315 KPIs $=721,935$ Results

Figure 4.30: The execution chain used to investigate the impact of 60 energy strategies across three designs and three energy scenarios. The first tree composes these 9 scenarios (see Section 4.6 (p148)), next scenarios are created which turn each energy strategy on one at a time, finally the integrated analysis tree developed in section 4.9 (p157) is used to analyse these 549 scenarios. The left of this figure shows the user interface for creating and configuring execution chains. 


\begin{tabular}{|l|l|}
\hline Anaerobic Digestion & Water Efficiency Fittings \\
- $13 \%$ Waste diversion & - $15 \%$ less Potable Water Demand \\
- $5 \%$ Energy Saving & - $3 \%$ Energy Saving \\
\hline Electric Vehicles & Black Water Treatment \\
- $3 \%$ Carbon Savings & - $45 \%$ less Waste Water \\
- $10 \%$ Reduction in Parking Spaces & - $10 \%$ less Potable Water Demand \\
- $6 \%$ Energy Saving & - Electricity Neutral \\
\hline
\end{tabular}

Table 4.1: The effects of particular energy strategies on CommunityB as calculated with Arup's IRM model and a one at a time sensitivity analysis conducted using HierSynth.

strategy which enables water re-use as grey water in irrigation is shown to be electrically neutral despite the energy costs involved in treatment.

This quantification of the impacts of particular strategies provides great value for designing a composition of strategies to be applied to a particular design under a particular scenario. Similar results can be useful for communicating to stakeholders the benefits of investment in a particular strategy.

\section{Model Analysis}

As well as looking at the effects of individual strategies it is useful to consider an overall model analysis of the results as this will give insight into the IRM model being used.

The most interesting result of this investigation concerns how many strategies were effective. By this we mean that they had an effect on at least one measured KPI across at least one of the 12 analysis models. The first thing we found is that the number of strategies that had effects varied according both to the design and the energy scenario ("Good", "Better" and "Best") considered. Whilst variation with the energy scenarios is implicit; variation with the design is less expected as it shows certain strategies will have no effect without certain design elements present in sufficient quantity (e.g. a certain GFA of housing).

Secondly, we found that 19 of strategies changed none of the KPIs considered; an insight highly surprising to the team. Upon investigation a number of reasons for this were identified as follows:

1. For $42 \%$ of these strategies HierSynth did not measure the correct KPIs to identify the strategy's effects. Mostly this was related to material consumption and waste, and was due to limitations in the set-up of the analysis. 
2. $16 \%$ of these strategies were not relevant to the CommunityB development and had been disabled.

3. $16 \%$ of these strategies had a negligible effect on several KPIs $(<0.001 \%)$ and was not measured.

4. Finally for $26 \%$ of these strategies (equating to $8.2 \%$ of all strategies) no effect was seen because of bugs from incomplete conversion of the IRM model to work at district level. After correction these strategies affected less than $2 \%$ change in carbon.

Similarly it was found that $28.4 \%$ of the KPIs measured across all models were not affected by any strategy although this is expected since changes to the IRM model will not affect all aspects of the DEF and LCCA models. As response to all of these insights refinement of the design scenarios, the IRM model and the HierSynth analysis tree were undertaken and resulted in more robust and accurate analysis.

\section{Design Space Analysis}

Finally we consider how this analysis helps the design and analysis teams consider the best ways to impact the design. We do this by considering the strategies with the most impact upon a KPI of interest. Further insight can be gained by identifying how these impacts vary with the design space in terms of the different land use designs and different energy strategies.

Fig. 4.31 (p195) presents the top ten strategies for impacting a single KPI under multiple designs and energy scenarios. It is interesting to note that the effects being measured in this table are the result of strategy effects in the IRM model propagating through several models in the integration shown in Fig. 4.9 (p160). Indeed the KPI shown is for the total operating costs of a Condenser Water Loop (CWL) district energy system as calculated by the final LCCA model of the integration. All strategies which reduce this KPI are shown under three designs of increasing density and the "Better" and "Best" energy scenarios. Note that the "Good" scenario does not appear in the table since it contains no district energy system.

From Fig. 4.31 (p195) we can discover much insight into the design space and means of impacting this KPI. First we note the substantial scope for more than halving the annual energy costs of the proposed CWL district energy system. These opportunities come from two disciplines (water and energy demand); with most impact coming from energy demand reduction but also a minority coming from water efficiencies. Whilst it is not surprising that reducing electricity consumption will reduce energy costs the impact of strategies to reduce water consumption is unexpected. This shows the utility of a multi-disciplinary Integrated Resource Management model [75]. 


\begin{tabular}{|c|c|c|c|c|c|c|c|}
\hline \multicolumn{2}{|r|}{ CWL Total Operating Costs } & \multicolumn{3}{|c|}{ Better } & \multicolumn{3}{|c|}{ Best } \\
\hline \# & Strategy & LU1 & LU2 & LU3 & LU1 & LU2 & LU3 \\
\hline & Er1 - Narrow Form Architecture & $7.31 \%$ & $7.07 \%$ & $6.62 \%$ & $10.95 \%$ & $10.60 \%$ & $9.89 \%$ \\
\hline 2 & Er6 - Night Time Plug Load Reduction & $7.31 \%$ & $7.07 \%$ & $6.62 \%$ & $10.95 \%$ & $10.60 \%$ & $9.89 \%$ \\
\hline 3 & Er8 - Active Electricity Saving Strategies & $7.31 \%$ & $7.07 \%$ & $6.62 \%$ & $10.95 \%$ & $10.60 \%$ & $9.89 \%$ \\
\hline 4 & Er9 - Server Efficiency & $4.00 \%$ & $3.77 \%$ & $3.40 \%$ & $7.79 \%$ & $7.54 \%$ & $7.06 \%$ \\
\hline 5 & Er7 - Day Time Plug Load Reduction & $3.64 \%$ & $3.53 \%$ & $3.29 \%$ & $7.31 \%$ & $7.07 \%$ & $6.62 \%$ \\
\hline 6 & Er3 - Hydronic Cooling & $3.64 \%$ & $3.53 \%$ & $3.29 \%$ & $7.31 \%$ & $7.07 \%$ & $6.62 \%$ \\
\hline 7 & Er4 - Existing Building Efficiency & $2.87 \%$ & $1.82 \%$ & $0.83 \%$ & $0.36 \%$ & $0.23 \%$ & $0.11 \%$ \\
\hline 8 & Wt4 - Water Efficient Landscape & & $0.20 \%$ & $0.31 \%$ & $0.64 \%$ & $0.80 \%$ & $1.26 \%$ \\
\hline 9 & Wt1 - Low Flow Flush Fittings & & $0.27 \%$ & $0.43 \%$ & $0.29 \%$ & $0.37 \%$ & $0.58 \%$ \\
\hline 10 & Wt2 - Water Efficient Appliances & & $0.09 \%$ & $0.15 \%$ & $0.14 \%$ & $0.17 \%$ & $0.26 \%$ \\
\hline 11 & Wt4 - Leak Reduction & & $0.11 \%$ & $0.17 \%$ & $0.11 \%$ & $0.14 \%$ & $0.22 \%$ \\
\hline 12 & Er5 - New Building Efficiency & & $0.27 \%$ & $0.58 \%$ & & $0.27 \%$ & $0.58 \%$ \\
\hline & Er2 - No Residential Air Conditioning & & & & & $0.13 \%$ & $0.31 \%$ \\
\hline & Maximum possible reduction & $36.10 \%$ & $34.80 \%$ & $32.31 \%$ & $56.83 \%$ & $55.58 \%$ & $53.29 \%$ \\
\hline
\end{tabular}

Figure 4.31: The most effective energy strategies in the IRM model for effecting the "Total Cash Operating, Thermal \& Electricity Costs" of a proposed district energy system for the CommunityB development. This KPI was chosen from 1,315 computed across the integrated model ensemble developed in section 4.9 (p157); sensitivities were computed under all nine scenarios generated in section 4.6 (p148).

It is interesting to see the decreasing effectiveness of the top strategies as density increases from LU1-3. This is exemplified by the decreasing effectiveness of "Existing Building Efficiency" measures from LU1-3 as more existing buildings are redeveloped. Correspondingly "New Building Efficiency" has an increasing impact between LU2 and LU3. This strategy is one of the nearly twice as many strategies $(\# 8-13)$ which are enabled by the increasing design density under LU2 or LU3 than under LU1.

However the addition of strategies \#7-13 do not quite compensate for the decreasing return of the most effective strategies, since the maximum possible reduction is still $10 \%$ lower under LU3 than LU1. This of course likely masks the fact that the system should already be cheaper to run percapita under the high density development. This reflects a pattern of diminishing returns, with greater returns possible under less dense (and thus less efficient designs) than under more dense (more efficient) designs where many more sophisticated energy efficiency strategies will be required each of which has less scope for impact.

Finally we see the importance of designing an effective energy strategy since the difference between the "Better" and "Best" energy scenarios is substantial - the total possible reduction is up to $65 \%$ higher under "Best" than under "Better". Note that figures similar to Fig. 4.31 (p195) can be generated for each of the 1,315 KPIs measured by HierSynth and should yield similar insight, none of which was possible before the use of the HierSynth platform. 


\section{Conclusions}

Despite the complexity of this analysis and the quantity of data it produces the sample of findings included here demonstrate the insight this approach can produce. Design space exploration coupled with integrated and automated models together with sensitivity analyses have produced clear insight which was not previously possible. This enables quantification of what may previously have been practitioner intuition enabling comparison and improved communication between stakeholders. There is clearly further potential to be pursued in each of these techniques and particularly in their composition.

Further work here could include the inclusion of more KPIs and particularly the exploration of different design space dimensions such as more construction phases or different climate and financial futures. Additionally detailed design insight such as breaking the analysis down by district may yield more insight to see which strategies should be applied where to gain most benefit and be most cost effective. Finally a deeper sensitivity analysis may be useful to identify the most effective level of implementation for each energy strategy (e.g. different levels of solar PV uptake). Another avenue for more insight would include more detailed modelling. One side of this discussion which is lacking thus far is a costing model for investment in these energy scenarios, the integration of such a model either deeply into Arup's IRM or as a separate model integrated via HierSynth would produce a discussion more interesting to the client. Finally in order to extract this insight it is critical that better means of post-processing analysis results are implemented and the potential for automated identification of trends and anomalous data could be employed for example through machine learning techniques.

\subsection{Collaboration Conclusions}

The research questions set out at the beginning of this collaboration were:

1. To identify whether the HierSynth framework could be applied to current practice and workflow with a view to addressing the mismatch between the design and analysis cycles discussed in Chapter 2 (p37).

2. To explore how more detailed analysis could be made possible using a computational framework such as HierSynth.

3. To understand the requirements, opportunities and challenges of applying a computational framework to generate insight for the project team by answering "what if" and design space exploration questions.

For the first of these questions HierSynth was quite successful, several analysis models were integrated into HierSynth and enabled the reproduction of existing figures and 
results in substantially less time than before, examples of this include Figs. 4.4 (p152) and 4.6 (p155). Over the course of the project these and similar new figures were generated over 11 times from different designs and different models enabling quantified results to be readily available to the design and analysis teams many more times than previously possible.

Several challenges were encountered in meeting this goal. Firstly model complexity meant that tools such as those described in Chapter 5 had to be employed to identify model inputs. Secondly automating these models exposed the model to more robust use than previously which exposed and introduced a number of bugs which were fixed with the project team.

One unexpected outcome of this automation of current practice was the close collaborative nature of the analysis work, since all models fed into each other and each team member was interested in the results of all models executed. Also being the person running HierSynth meant that the author gained insight and an overview of all of the different models and practices involved in the analysis team allowing a wider perspective than a single discipline might have. There appears to be scope for computational frameworks to help improve workflow patterns and make collaboration simpler, further investigation of this should prove interesting. As evidence of improved collaboration we cite the length of discussions from practitioners across multiple disciplines who came together to study the results of HierSynth, model runs, modelling integration and design space investigations; these discussions were always valuable to all concerned. For example several opportunities were identified for practitioners such as the opportunity to benchmark models against each other or to try to put a quantitative value on an intuition. Other valuable insights included win-win situations such as verifying that water saving strategies also reduced energy consumption - something which was previously an intuition.

The second challenge was to improve current practice within the analysis team. This included the greater analysis resolution HierSynth's hierarchical analysis provided. Similarly analysis over time provided more accurate results and the capacity to model trends over time which was previously not possible. Finally section 4.9 (p157) describes the process and challenges of integrating three previously separate models together into one more powerful model. Though integration and the model adaptation and verification was often a substantial challenge this integrated model was more accurate than previous models and formed the basis of the insight generated in the remainder of this chapter and enabled the investigation of the design space which was the third research question.

The exploration of different scenarios was perhaps the most successful part of this collaboration and although at times challenged by model robustness or large requirement's for data the investigations detailed in the last half of this chapter have shown 
the exploratory and explanatory power of a computational framework automating and integrating analyses with scenario composition and hierarchical analysis.

\subsubsection{Top Five Capabilities}

To summarise the contributions of the HierSynth platform to the case study project we identify the five most important capabilities enabled by the HierSynth framework:

- Integrating three previously separate models into one using HierSynth and improving model robustness for each in the process.

- Enabling the comparison of multiple designs under multiple scenarios under many KPIs.

- Enabling more detailed analysis than previously possible in a hierarchical manner from block to district to development scale.

- Enabling accurate modelling of trends in assumptions over time.

- The identification and resolution of multiple model bugs mostly relating to robust input handling resulting in a more robust and accurate model.

\subsubsection{Top Five Findings}

These capabilities enabled a number of insights into the project which would have been difficult or infeasible to achieve without the computational support provided.

- Quantifying the effects of increasing density and varying carbon mitigation strategies on the masterplan.

- Investigation of the impact of electrical cars on energy demand and carbon emissions showing the increasingly strong case for this as the development is made more dense and more sustainable.

- That contrary to expectations the trend for percapita transport carbon emissions is not $\cup$ shape but $\sim$ shaped and exploring the reasons for this.

- That a $30 \%$ residential, $70 \%$ commercial development is the key ratio for district energy system performance in the CommunityB development. That this is also the key point for reducing transport carbon emissions and that the same effects are possible by adding $35 \%$ residents to the existing design under discussion.

- Enabling 3d visualisation of analysis results in a fly through environment which enabled improved communication with stakeholders. 


\subsection{Framework Evaluation}

In this section we consider the effectiveness of the techniques and data structures employed in this collaboration. From the outset a major research question of this collaboration was to identify the practical utility of a number of techniques as implemented in the HierSynth framework:

Specifically:

- Whether simple model automation can reduce the gap between the analysis and design cycles.

- To explore the potential of scenario composition to generate design insight.

- To consider the effectiveness of model integration and the difficulty involved.

- To identify whether the HierSynth execution chain model and the HierSynth trees it contains can be effectively mapped onto the design and analysis challenges within the project. Particularly the applicability and utility of the design decomposition it enables.

- To show the potential of 3d visualisation and integrated analysis within the urban masterplanning teams.

- To identify how effectively performance trees can be used to visualise performance across a design and its decomposition by showing multiple KPI's from many disciplines.

- To explore the effectiveness of sensitivity analyses upon the models used within the project.

We will now discuss these questions together with other aspects of the collaboration and HierSynth framework. During the project a number of unexpected problems arose and necessitated further development of the HierSynth framework (e.g. execution chains) alternately some of the techniques expected to be the most useful proved not to be so (for example performance trees). Finally we encountered a number of opportunities for the exploration of other techniques not currently integrated into the HierSynth platform and we will conclude this chapter by discussing these.

\subsubsection{What Surprised us}

By working closely with a live project team a number of the techniques employed by the HierSynth framework turned out to be more useful than expected at the outset. 
- Effectiveness of Automation - Having automated the models used in this collaboration it was surprising to see the amount of value generated to the project team. This took several forms from simply automating production of complex graphs and so removing the need for a time consuming task to reducing the lead time for such graphics (e.g. Fig. 4.6 (p155)) to be generated enabling quicker analysis and feedback upon new design iterations. Similarly more analysis iterations were enabled allowing greater investigation of the design space.

- Detailed Analysis - There was a surprising amount of insight to be gained from providing a more detailed analysis to the design team for example Fig. 4.7 (p156) breaking performance down by district and construction phases identified a range of model bugs and issues to be resolved in the design.

- Need for Model Comprehension - Initially I had expected that each model integrated into HierSynth would and could essentially be treated as a "black box" with a simple list of inputs to enter and list of outputs to read. However it was soon found that in order to gain coherent insight into results it was necessary to have a clear understanding of how each model performed internally, particularly with the need for identification and fixing of model bugs which are common to all large complex models. This challenges has two halves, first in the initial automation of an analysis model which requires deep comprehension of the model. Once a framework like HierSynth is in use a second challenge will arise; this surrounds ensuring the correct use of the model, understanding the implicit assumptions within the model and the calculation methods it employs. Both of these challenges were encountered within this case study and must be further addressed in future work.

- Model Complexity - This was an order of magnitude more than expected. Although I had worked with previous version of the IRM model, the latest version of the model in use on this project presented a substantially more complex analysis model particularly with the energy scenarios integrated throughout the model. More discussion of this complexity can be found in Chapter 5 (p209).

- Multidisciplinary Nature of Investigations - Over the course of the collaboration I have by osmosis and necessity gained a shallow insight into many different disciplines involved in the assessment of urban masterplans. This need for a wide a general knowledge of many disciplines was not something I had expected to need at the outset of the collaboration. As an example section 4.12.3 (p177) shows an investigation in the effects of residential and commercial mix on the masterplan. To investigate this knowledge of and input from many disciplines was required, from masterplan design, through energy demand modelling, district 
energy system design and costing and transportation modelling. Perhaps partly this steps from nature and strengths of the IRM model and the many disciplines and resources it integrates, however I suspect it is a feature of the AEC industry more generally.

- Complexity of Integration - Given the above it is not surprising that the complexity of the integration described in section 4.9 (p157) was more than expected. The main challenges were not technical rather they surrounded understanding and harmonising assumptions between the three models and resolving modelling differences between them which required a deep understanding of model function for example to avoid double counting of energy demands.

- Effectiveness of Sensitivity Analysis - Whilst I expected a PB analysis to prove useful in identifying the top few factors with scope to affect carbon emissions (see section 4.13 .1 (p187)) I was surprised by the volume of data and insight produced by the far simpler one at a time analysis of 60 energy strategies (see section 4.13 .2 (p190)). This gives great emphasis to the view that the best use of computation is not in optimisation but rather in design space exploration enabling the creativeness of designers and engineers. We see that sensitivity analysis provides a map to the design space and so provides stimulation not simulation.

- Model Debugging - Finally the biggest challenge was the requirement for model debugging. Almost every investigation into the design space turned into a debugging exercise into the models. This is not to invalidate the model's normal use, rather as computation-enabled exploration of the design space in new directions the model was exercised in ways it is not normally used. The discipline specific and cross-disiplinary working required for this as well as comprehension of complex models presented a substantial challenge and consumed much time. To address this a number of tools were developed to investigate the construction of Excel Models and can be found in Chapter 5 (p209).

\subsubsection{What Worked}

- Automation - HierSynth performed well with automation tasks, particularly after a move to 64bit architecture allows large memory addressing, over the the course of the collaboration an estimated 100,000+ model runs were completed.

- Integration - was very successful in producing more accurate results and a model with greater capacities. While not technically difficult some care had to be taken when introducing linked execution of models into the parallel execution. 
- Multiple Scenarios - It was surprisingly effective simply to run different scenarios for example the ten construction phases discussed in section 4.8 (p153).

- Scenario Generation - The capacity to compose scenarios both within a scenario generation tree was very effective and used throughout the collaboration. This feature was developed during the collaboration and is discussed in section $3.11(\mathrm{p} 120)$.

- Execution Chains - The execution chain concept was developed during this collaboration and was then used throughout. It enabled the construction of large design space exploration experiments along with sensitivity analysis runs. It also enabled the re-use of analysis trees such as the one in section 4.9 (p157) through out the project. Example execution chains used in this collaboration are shown in Figs 4.17 (p173) \& 4.30 (p192).

- Design Decomposition - Whilst design decomposition was only done via the districts and buildings of the development it did give rise to useful insight into design performance such as the percapita carbon maps shown in Fig. 4.11 (p163). With more design detail this method should give greater insight.

- Sensitivity Analysis - Both PB and a simple one at a time analysis were simple to set up using the HierSynth framework and yielded great insight especially composed with design space exploration such as in Fig. 4.31 (p195).

- Expression language - The NCalc expression language used throughout HierSynth provided very flexible customisation of scenarios, integration and analyses; enabling quick changes to analysis runs and execution chains, e.g. enabling the saving of particular model instances under certain design scenarios for debugging purposes.

- Performance - Whilst it could always be faster, analysis times provided by HierSynth were always workable and meant results could normally be returned to the analysis team within an afternoon.

\subsubsection{What Didn't}

Unfortunately some of the techniques were not as important or effective as originally expected.

- Performance Trees - Whilst conceptually showing great promise of giving simple and effective cross-disciplinary insight into design component, performance trees were not generally used. They were found to be too complex to set up particularly when complex aggregation of different units of performance was required. 
However the one case in which they did perform well was in $3 \mathrm{~d}$ visualisation environments where a performance tree was geolocated over each design element (e.g. Fig. 4.16 (p170) per city block) where they enabled simple visualisation of analysis results although again the composition of performance was challenging. It is possible that the automatic composition of performance trees might be possible using machine learning techniques, for example by learning which metrics a practitioner normally accesses then tracking the normal ranges of such KPIs and building performance trees to show anomalies. Another reasons for the poor impact of performance trees is the way analysis results were used. A normal usage pattern was for engineers to look in detail through the results exploring performance across 50-150 KPIs or looking for trends which is more easily done looking through a table than a performance tree. Creating performance trees containing this number of KPIs would have been a challenge, particularly given the difficulty of creating preference functions or aggregators.

- 3d Visualisation - Whilst the case study discussed in section 4.11 (p166) shows the potential of automated analysis with $3 \mathrm{~d}$ visualisation a lack of project specific geometry and data precluded meaningful analysis result to the CommunityB development. Particularly lacking were different geometry for design alternatives which would show the physical trade-offs the changes in design would entail. Additionally it is a necessity that the analyses displayed in a $3 \mathrm{~d}$ environment are actually geometric in nature. There is limited value in displaying non-geometric analysis results in $3 \mathrm{~d}$.

\subsubsection{Additional Computational Needs}

Having completed this collaboration a number of techniques and features can now be seen to necessary for successful projects in the future.

- Error handling - Given the challenges in integration, poor error handling in the HierSynth framework was not helpful and should have been given greater consideration at design time. Particularly required are techniques to highlight unusual, outlying and error-state results from analyses.

- Debugging tools in HierSynth - Whilst during the collaboration some code was added to save a particular model instance during execution, the ability to re-run and debug a particular model run would be highly useful as it would enable interactive debugging without needing to wait many iterations of long running analyses.

- Version Control - The number of model versions received over the course of the collaborations was substantially larger than the expected static model. Over 
nine months some 23 versions of the IRM model were received which reflected changes in energy strategy design and a number of iterations of bug fixes. Version control would have ensured that all team members would have the same up to date analysis models since this was a frequent source of problems when model transfer was done via multiple e-mails. Version control should also enabling of branching of models to enable customisation for particular investigations such as those discussed in section 4.12 (p171). Intelligent merging of models would enable incorporation of bug fixes across models and enable harmonising of assumptions between design space investigations. This would however require deep insight and change identification within the models employed which may present a difficult challenge. Similar problems also occur within the design data iterations and design scenarios; a proper version control system should reap similar benefits particularly if a distributed version control is employed across different teams and firms such as the design team where updates could be automatically pushed to analysis engineers. Finally consistent versioning of analysis results is also key to presenting correct results.

- Provenance Tracking - Enabled by proper version control provenance tracking enables identification of which results were generated from which set of designs, scenarios, models and HierSynth trees. This information is of course encoded within a HierSynth tree at run time, however persisting this information via proper versioning and a provenance store would reap substantial benefits not least in being able to check how results were processed into a final graphic or insight. This is particularly a challenge with multiple discipline's models and practitioners in an area where no one team member will understand the models and methods used across the whole team. Other benefits would include being able to redact only those results which depend on erroneous data or models should they be discovered. A final benefit of this type of tracking is that it enables identification of the most common uses of each model alone and in concert, this enables identification of good targets for investment in model improvement, integration and automation. This idea is developed further in [88].

- A Collaborative Environment - Given the number of practitioners and disciplines from several firms in many geographic locations (and continents) it is unfortunate that HierSynth is not able to be used by multiple users. A collaborative environment enabling each practitioner to modify their model, design or scenario separately, set up new analysis runs and analyse results within the same environment would be very valuable. Collaborative investigation of results and trends would also be value. This would of course require good version control and provenance tracking. 
- User Friendly Interface - Currently HierSynth is an expert tool and not amenable to use by all practitioners involved. Development of a simpler userinterface and possibly a simpler data model would enable each practitioner to generate automated results and to undertake investigations such as those discussed in this chapter. This would also enable greater investigation of analysis results and the identification of greater insight, something which was difficult with current tools. However challenges would be faced in user training and uptake.

- Data visualisation tools - Graph creation was only semi-automated within HierSynth. Much post-processing was done using Microsoft Excel. The integration of full reporting and data visualisation tools would provide interactive exploration of the data and enable other team members to explore and identify new trends and an insight. The primary difficulty in this is dealing with the large number of data dimensions, be it levels of the design decomposition, different designs, energy strategies or construction phases. Additionally the number of data types and the post-processing they require (e.g. to identify change to a base scenario) make creating a flexible system a difficult challenge, though one which should yield considerable benefit, not least in the identification of trends and anomalous results. However this should be balanced by the knowledge that most final graphics required tweaking by the analysis team and that more valuable than "perfect" final graphics being automated was access to the raw data. Hence we envisage data visualisation tools being used within HierSynth primarily to quickly explore large quantities of data or for automatically producing commonly required figures.

- Machine Learning - Given the wealth of data generated on each design and the number of explorations into the design space made in this chapter there is a strong case for the incorporation of machine learning algorithms into HierSynth to automatically identify anomalous results and trends so that they may be flagged up for practitioners to consider. This would ideal if integrated into the design decomposition trees. For example there are some 750, 000 data points generated in section 4.13 .2 (p190) out of which insights such as Fig. 4.31 (p195) need to identified. This could be enabled by comparison amongst design element peers across different designs and scenarios.

\subsubsection{Areas for Further Investigation}

In addition to these areas there are number of areas and techniques which we can see potential benefits from. A selection of these would be:

- Deeper Sensitivity Analysis - Given the success of the sensitivity analyses performed in sections 4.13 .1 (p187) and 4.13 .2 (p190) a deeper more insightful 
sensitivity analysis module should yield greater insight. Two features especially would be useful. Firstly an analysis which uses multiple levels for each input would help in the design process by allowing finer comparisons to be made. Secondly given the complex and interrelated nature of many of the analyses and the inputs to them an analysis which can consider more of the interactions between the effects of inputs would be highly useful. This would avoid aliasing effects and help identify where two factors in concert provide a greater benefit than either in isolation. These improvements would however come at a computational cost which would need to be addressed.

- Cloud Computation - Whilst the analysis times during this collaboration were normally within one hour it is easy to see how more detailed analyses, deeper investigations or more complex analysis modules would lead to computational demands which a single machine could not meet. To address this offloading some analyses to other machines in either a local private cloud or an external cloud compute provider such as Amazon's Elastic Compute Cloud [1] would result in faster analysis times. This of course depends upon parallelisation of work units - something which the HierSynth analysis trees have proved good at (see section 3.7 .2 (p105)). Issues that would have to be addressed to implement this include licensing of analysis engines and data privacy.

- Computational Optimisation - Additional compute power has often been used for computational optimisation of designs. This is a hazardous area for several reasons. Whilst computational optimisation is a very successful technique in constrained environments such as sizing a steel frame, the wider the design space and the number of constraints involved limit the effectiveness of computational optimisation. Thus using computational optimisation for general urban masterplan design would prove particularly difficult given the size of the design space and the number of often informal relations between the many design assumptions made across many different disciplines. Finally difficulty will be incurred in the geometrical nature of the design to be created. This said however the addition of a computational optimisation module into a HierSynth execution chain would have clear benefits if used to optimize particular limited aspects of the design such as the level of a particular energy strategy compared to its cost. These warnings given this is an interesting area of active research and of course even sketchy computational design could be seen as a way of traversing the design space more quickly than otherwise possible and perhaps discovering insight in the journey or at unexpected ends.

- Informal Ontology Development - The number of inputs, units and data types encountered in this project has been substantial. An ontology acts as a 
means to store formal meanings for each of the data points and their type. This allows formal checking that the correct units have been used throughout the project. Unfortunately the variety and quantity of data in projects such as this precludes creating an ontology formally before the project. However if such an ontology could develop informally from structured comments and variable naming together with autocompletion many of these benefits could be gained with little of the cost.

- Ontology based integration - The use of such ontologies as well as those already created as data [42] [55] [3] may provide a simpler way of automatically integrating models enabling simpler connection of data between models since the framework would know which data points are outputted from one model and can be inputted into the next. Similarly it could be ensured that correct units are used between models - something that caused some problems during the integration described in section 4.9 (p157). Some feature of the HierSynth framework, such as the automatic extraction of analysis module requirements and outputs via the internal expression language begin to provide the background techniques for such a system (see section 3.6.6 (p100)) and this should be developed further. 
This chapter has shown the benefits to a masterplanning project from the application of a computational framework such as HierSynth. We have identified a number of successful techniques and features of a data model that helped to achieve this insight. We have also seen several techniques which were not as successful as hoped and considered a number of areas where new techniques are much needed. The biggest of these is clearly for better tools for debugging and analysing the complex models involved in this case study. Tools such as these are developed and discussed via a case study in Chapter 5 (p209). 


\section{Extraction and Analysis Methodology}

Having identified that model robustness and debugging capacity is a major challenge in applying computational techniques to urban masterplanning this chapter demonstrates a methodology to help engineers and designers maximise the utility of complex multidisciplinary engineering models. As motivation we investigate the expanding use of Integrated Resource Management (IRM) models which enable urban masterplans to be assessed for sustainability metrics across many disciplines. IRM models reflect the inherent complexity of multidisciplinary sustainability analysis by integrating models from each discipline. This complexity makes their use time-consuming and reduces adoption and adaptation for projects. This was particularly found to be the case during the use and subsequent integration of the IRM model with other models as described in section 4.9 (p157).

To address this, a toolkit was created for analysing multidisciplinary engineering models implemented as spreadsheets. For a given output, a relevant slice of the model is extracted, visualised and analysed by computing model and interdisciplinary metrics. A sensitivity analysis of the extracted model supports engineers in their optimisation efforts. These methods help expose, manage and reduce model complexity whilst giving practitioners insight into multidisciplinary model composition. This aids assessment and design optimisation work between and across disciplines.

This chapter considers several generations of Arup's IRM model and details the insight generated, for example identifying which of 933 input parameters has most scope for reducing percapita carbon emissions and showing side effects. The methodology and toolkit are more generally applicable to other engineering models beyond IRM models and have been applied to a vertical transportation model. This methodology address many of the areas for further computational support identified in section 4.15 .1 (p199).

\subsection{Introduction}

To demonstrate the challenges of multidisciplinary engineering models we consider models with the urban masterplanning community. Increasing requirements for managing environmental impact have led to demand for interdisciplinary modelling of sustainability metrics, such as annual percapita carbon emissions, in order to benchmark and improve designs. These drivers have been unified by Integrated Resource Management 


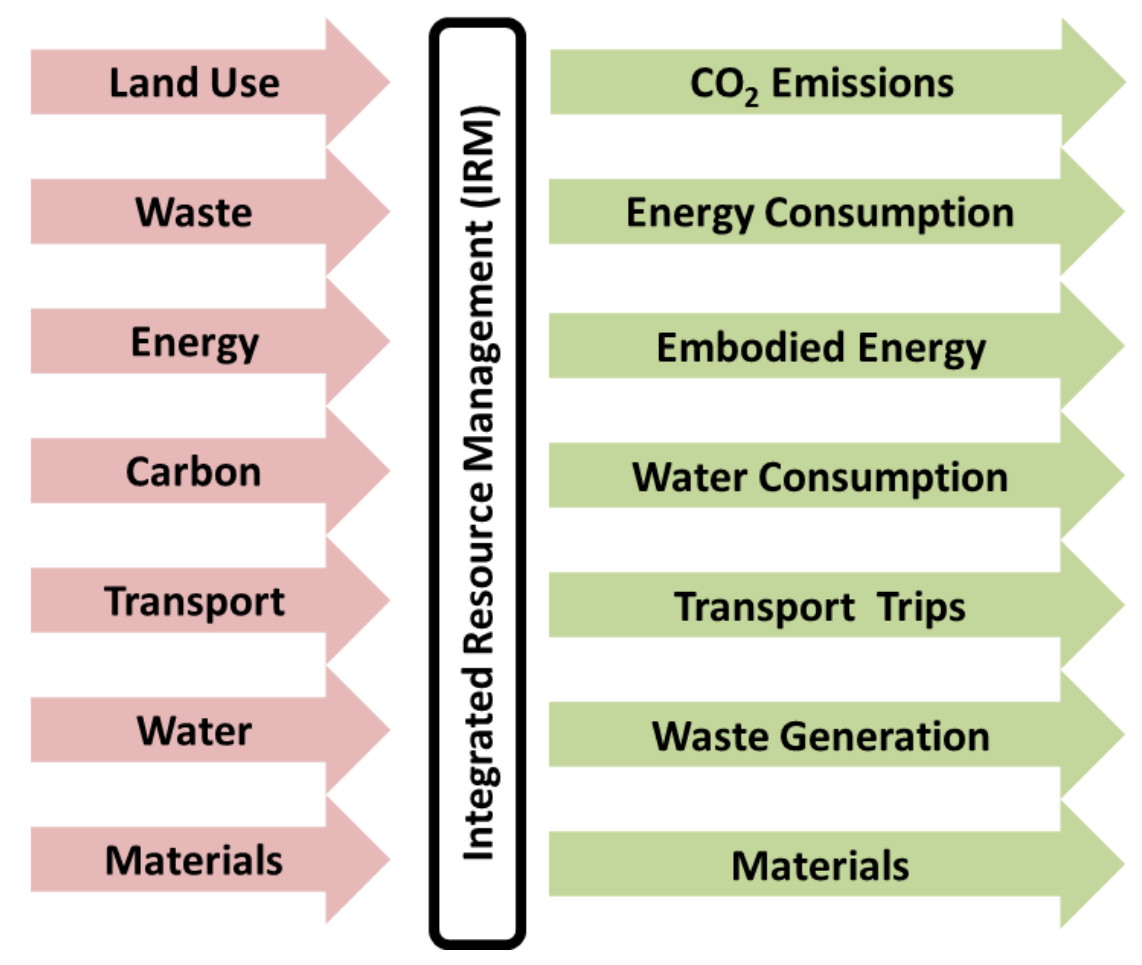

Figure 5.1: Conceptual model of an Integrated Resource Management (IRM) Model [12]. Sustainability models from many disciplines are integrated to form a coherent model for assessing urban masterplans [75].

(IRM) [12] [75] [54] models which integrate sustainability models from each discipline into a coherent assessment tool. Such models are commonly implemented in spreadsheet form, for ease of construction, modification and portability. While many benefits are realised by an integrated model there are some inherent difficulties common to many engineering models; these motivate this work, stemming from the complexity of the models and are discussed in the next section. In this chapter we demonstrate the value of computational model analysis tools in resolving these challenges by aiding practitioners comprehension, modification and use of their models. Particularly:

- We present a tool suite for systematic, automatic analysis of large spreadsheetbased models, and a methodology for using such tools to enhance the quality and value of spreadsheet-based modelling.

- We present static analysis tools, incorporated into this framework, that help identify problematic aspects of the model, and to help diagnose defects.

- We present a sensitivity analysis tool for large spreadsheet-based models, and illustrate its value in identifying the key parameters in Arup's IRM model with respect to selected performance indicators.

- We demonstrate the potential for these static analysis tools by exploring three 
versions of Arup's IRM model at different stages of development. Showing insights into these models such as identifying a potential bug in the transport carbon calculation, identifying several structural features of the model that might be improved and confirming whether or not several disciplines communicate with each other.

\subsection{Motivation}

In this chapter we consider Arup's IRM model [12] [75] as an example of a complex interdisciplinary engineering model. As such, we now consider some of the difficulties inherent to such models. More information on this particular model can be found in section 4.4.2 (p145).

As shown in Fig. 5.1 (p210), Arup's IRM model consists of several different discipline sub-models including energy demand, energy supply, passenger transport and land-use, each of which has a data input model and a calculation/output model giving a variety of sustainability metrics such as annual water supply figures. These input/output model pairs strongly rely not only upon each other, but also upon the other discipline's input and output models. For example the energy supply sub-model uses inputs from the land-use input sub-model and the outputs of the energy demand model.

This leads to a complex interrelated web of calculations which reflects the physical nature of sustainability concerns as shown in Fig. 5.2 (p212). This figure shows a conceptual model of the IRM model, the interrelation of the discipline specific submodels and their responsibility to provide results to each other. In addition to these interlinked discipline models, a project-specific sustainability dashboard is integrated which calculates summary metrics such as annual percapita $\mathrm{CO}_{2}$ e (equivalent) emissions which uses information from nearly all of the discipline input and output models. This complexity is a requirement of faithful modelling, however it leads to challenges for the engineers who use the model as follows:

- Model Complexity - As Fig. 5.2 (p212) demonstrates, an IRM model by its nature is complex due to the strong coupling between already intricate discipline models. This leads to difficulty in gaining an accurate overview of the whole model and also to understanding how a single discipline's model functions, especially if this model is from a discipline other than the practitioners' field of expertise. For example this was the case when the author came to automate the IRM model for use by HierSynth.

- Data Requirements - Due to the scope of urban masterplanning and the inherent complexity of sustainability analysis, an IRM model and indeed many engineering models require large amounts of data to function. Our analysis iden- 


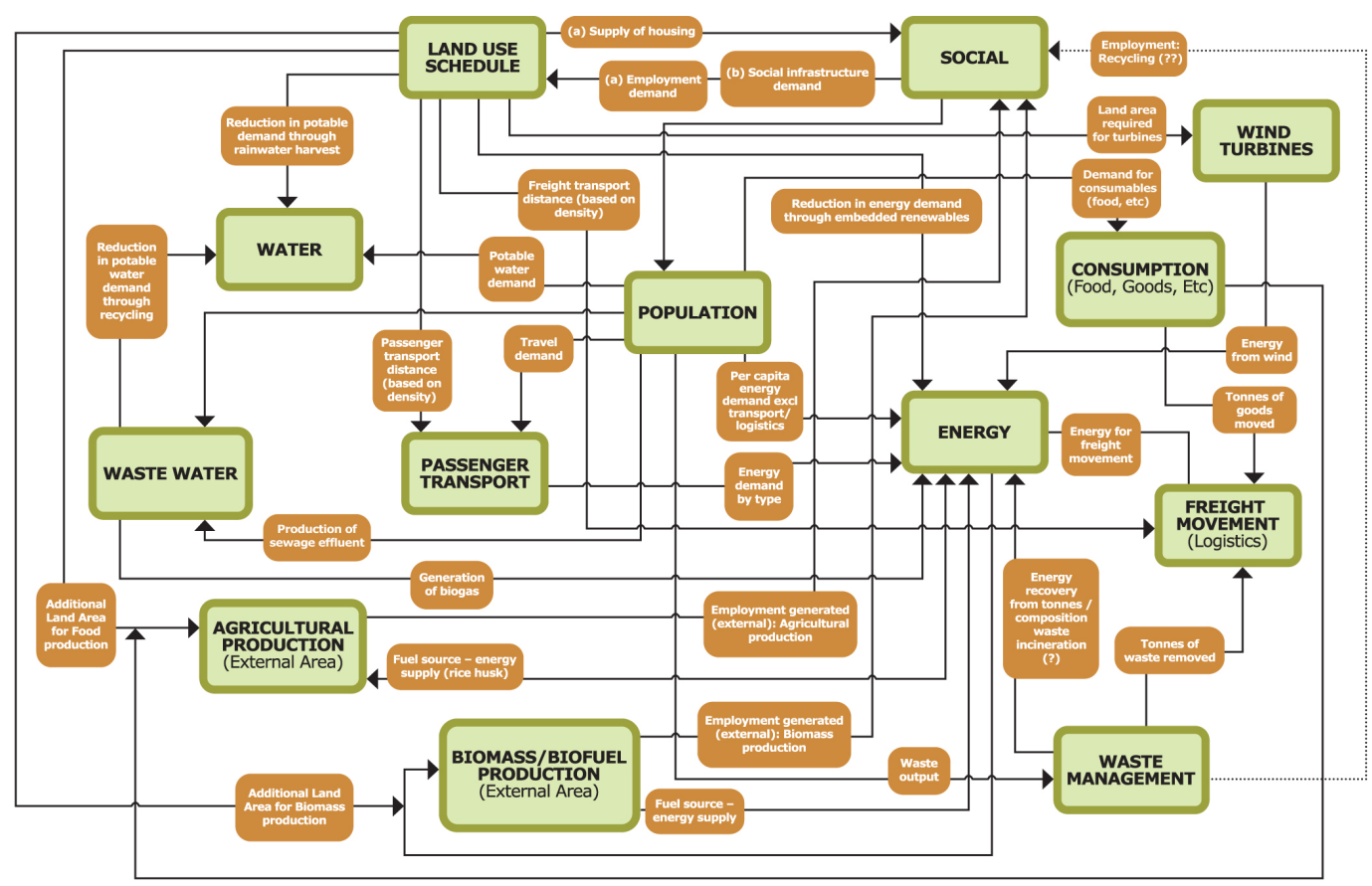

Figure 5.2: Dataflow through Arup's IRM model [75]. The complexity of multidisciplinary engineering models and difficulty of comprehension makes project adaptation and optimisation tasks challenging.

tified 933 separate design or analysis variables which must be entered within the carbon calculation of Arup's IRM model, ranging from the total floor area of residential buildings to the $\mathrm{CO}_{2}$ e emissions for disposing of a computer monitor. The time taken to gather, process and enter the required information is likely to be the largest time cost in applying an IRM model to a new case study. These challenges were also faced during the automation of the model as dicsussed in section 4.5 (p146).

- Implicit Knowledge - Much of the knowledge within many of the disciplines involved in an IRM model is difficult to formalise, being built up as an informal set of good practices over time. During the modelling process attempts are made to formalise some of this implicit knowledge by determining data ranges and variations between design iterations. This is a challenging process particularly if formalised knowledge is not clear within the model [105] such as is common in spreadsheets where formulas are not explained.

- Interdisciplinary Communication - Each discipline within an IRM model has its own nomenclature which must be made clear to the other disciplines involved. It is thus easy for two disciplines to require an estimate of a figure under two different names under different nomenclature and in two different units leading 


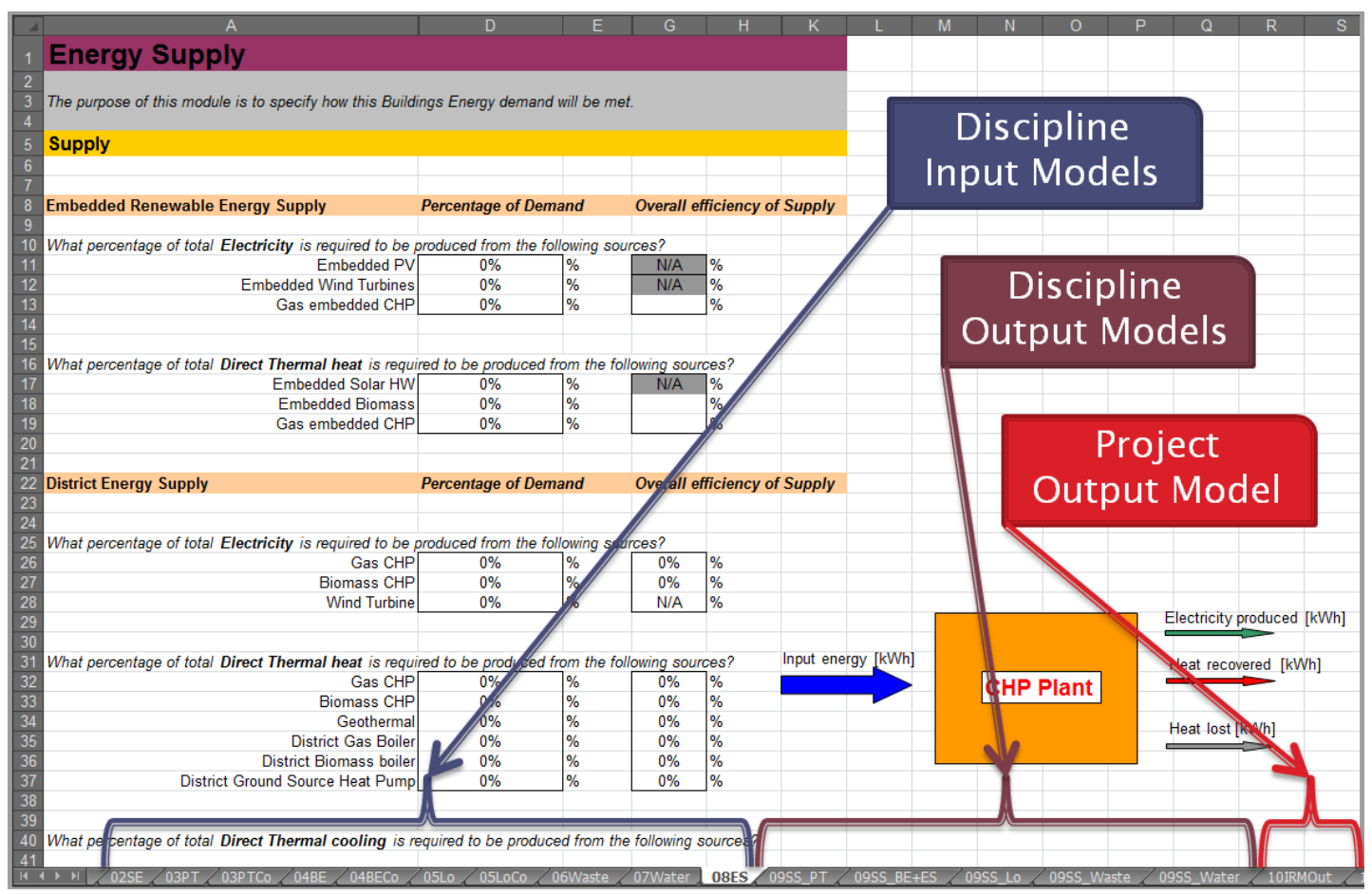

Figure 5.3: Arup's IRM model [75], is implemented as a Microsoft Excel spreadsheet. Each discipline has an input and an output model in its own worksheet. A single project metrics dashboard is provided. The IRM model contains over 20 submodels and some versions of the model contain over 50,000 calculation cells. 
to model inconsistencies.

- Project adaptation - Most engineering models, whilst trying to be as general as possible, often require some tweaking to fit the exact nature of the task at hand. Due to its scope an IRM model often requires adaptation to each project for the following reasons:

- Models too broad - A model's data requirements are large and can prove broader than the scope of the project especially during the early design stages. This leads to difficulty in fulfilling all the data requirements.

- Models too narrow - Counter-intuitively a common cause of model adaptation is to meet project specific concerns. For example the inclusion of irrigation and grey water recycling is critical in water stressed areas but is rarer in more temperate climates and so may need to be added to the model.

- Cause and Effect unclear - Project adaptation for these reasons is a difficult activity - the scale of the model can make identification of cause and effect between an input to be modified and the final sustainability metric difficult to determine, especially because of the interrelated nature of IRM models.

- This was particularly shown in section 4.9 (p157) during the integration of the IRM model with other models. The difficulty of establishing cause and effect made correctly identify the parts of the model to integrate and automate a challenge. During the integration much model adaptation was required both to remove functionality not used in subsequent models and alternatively to add functionality which was present but would be required by subsequent models in the chain.

- Difficulty of Optimisation - Once a design has been assessed a number of design improvement recommendations are normally made. This is a difficult process as it depends on understanding both the overview and the detail of the whole IRM model and its constituent sub-models, making best use of the high levels of implicit knowledge involved in setting the large data requirements as well as understanding the flow of cause and effect across the numerous discipline models to identify the handful of most advantageous steps that could be taken to improve the design.

- Implementation - As with many other engineering models, Arup's IRM model (shown in Fig. 5.3 (p213)) is implemented within a Microsoft Excel spreadsheet. This gives wide portability and simplicity of model change for a large user base. However it makes understanding of the interrelations and cause and effect within the model difficult. Indeed there is a growing body of evidence that spreadsheet 
models in common with other large software products are likely to contain errors at unacceptable rates. A good summary of the current evidence is available in [77].

In summary there are clear obstacles precluding the widespread usage of IRM models. These problems are not only common to IRM models but to complex multidisciplinary models in general. This chapter demonstrates that one solution is to apply computational methods to such engineering models in order to support practitioners in their information intensive tasks, helping tackle the problems identified in this section.

\subsection{Methodology}

As detailed in [61] with application to the design process we propose and demonstrate an Extraction and Analysis Methodology (EAM) consisting of a series of techniques to help expose, reduce and manage model complexity and give insight into multidisciplinary model composition and aid designers in focusing design effort in optimisation tasks.

The methodology has the following steps:

1. Obtain - Model and project objectives.

2. Define - Key Performance Indicators (KPI)'s of interest to the project.

3. Extract - Slice model to expose and reduce complexity to produce a smaller model computing only the KPI's of interest.

4. Analyse - Visualise - Visualise model to aid comprehension and show cause and effect.

5. Analyse - Metrics - Compute metrics on calculation model to give insight into model composition.

6. Optimise - Set variable ranges to formalise implicit knowledge enabling sensitivity analysis to give insight and focus optimisation effort.

The input to this process is the engineering model (possibly without project specific data) and an indication of which outputs of the model are of interest to the designer.

\subsection{Related Work}

This work follows a growing trend of applying software engineering techniques to climate and sustainability models [30]. These models are inherently large complex software structures. In this case we consider spreadsheet based models which are not frequently considered as complex software models. 
A number of studies have identified the presence [77] [26] and the frequency [18] of spreadsheet errors. From controlled studies we know that error are common amongst novice and expert modellers [51]. We also know that the majority of modellers do not have formal training in spreadsheet based modellers [77]. A body of literature has developed aiming to formalise a taxidermy of spreadsheet modelling bugs [78]. Some of these bugs are unique to the environment of spreadsheet modelling whilst many are in common with other programming environments. The risks of these errors are very commonly underestimated and few users of spreadsheets consider the risks of such errors [18]. Indeed very few practitioners consider that they need tools for debugging their models.

There have been a number of studies into auditing tools for spreadsheets (e.g. [18]) (for example for tax purposes) together with tools to identify areas of complexity within spreadsheets [20]. Historically there has been much interest in deriving visualisations based on the calculation graph of a spreadsheet [52] [90]. Several visualisation tools have been proposed to avoid costly errors. The relationship of each of these approaches to different areas of the methodology we propose will be discussed within the relevant sections of this chapter.

The novelty of our approach is that rather than treating a spreadsheet as simply a software artefact we consider the insight each step and tool in our methodology can generate for the model maintainer with a view to aiding them as they optimise a design. This is particularly a challenge for engineering models as appose to financial models which have previously been the focus of research. These engineering models through their constant evolution and adaptation to projects present many new challenges to the area of spreadsheet based modelling. In contrast to much previous work where techniques proposed are done so in isolation without an overarching methodology for their use. We also consider for the first time the challenges that a multidisciplinary model brings to the challenge of spreadsheet engineering. Together with new approaches for assessing multidisciplinary communication within models (e.g. section 5.8 (p221) and 5.8.1 (p222)). We also consider how sensitivity analysis may be performed in large spreadsheet based models, this is enabled through our extraction and analysis methodology and has the potential to generate substantial insight for practitioners as evidenced in section 5.11 (p229). Finally for the first time we consider the evolution of complex models as they are developed and applied to projects. As discussed in section 5.12 (p232) this is a substantial source of insight into the model.

\subsection{Model Extraction}

In order to give insight into the model we extract a slice of the model from Excel. Slicing the model or computer program is a well known technique [104] which allows the user 
to consider only the portion of the model involved in the calculation of the particular output metrics which are of direct interest, reducing the model size and complexity.

We recursively extract from the model by starting from the outputs of interest (such as annual percapita carbon emissions), reading their formula parsing them for references to other cells, reading these cell's formula and so on recursively until no more cells are referenced. In order to do this we used mathematical expression evaluation library NCalc [85] and modified the grammar to be compatible with Excel formulas and implemented a subset of the Excel functions. In contrast with many other approaches [83] [90] [52] this formula parsing approach enables us to gain insight within formulas, for example differentiating cells referenced from table lookups which reference hundreds of cells from arithmetic references. This enables simplification of the extracted model slice and resultant graph of cells. To ensure correct extraction we re-run the calculation in the expression library and check against the Excel calculated results. We also extract all values and custom cell names to aid comprehension of visualisations, metrics and sensitivity analysis runs.

\subsection{Visualisation}

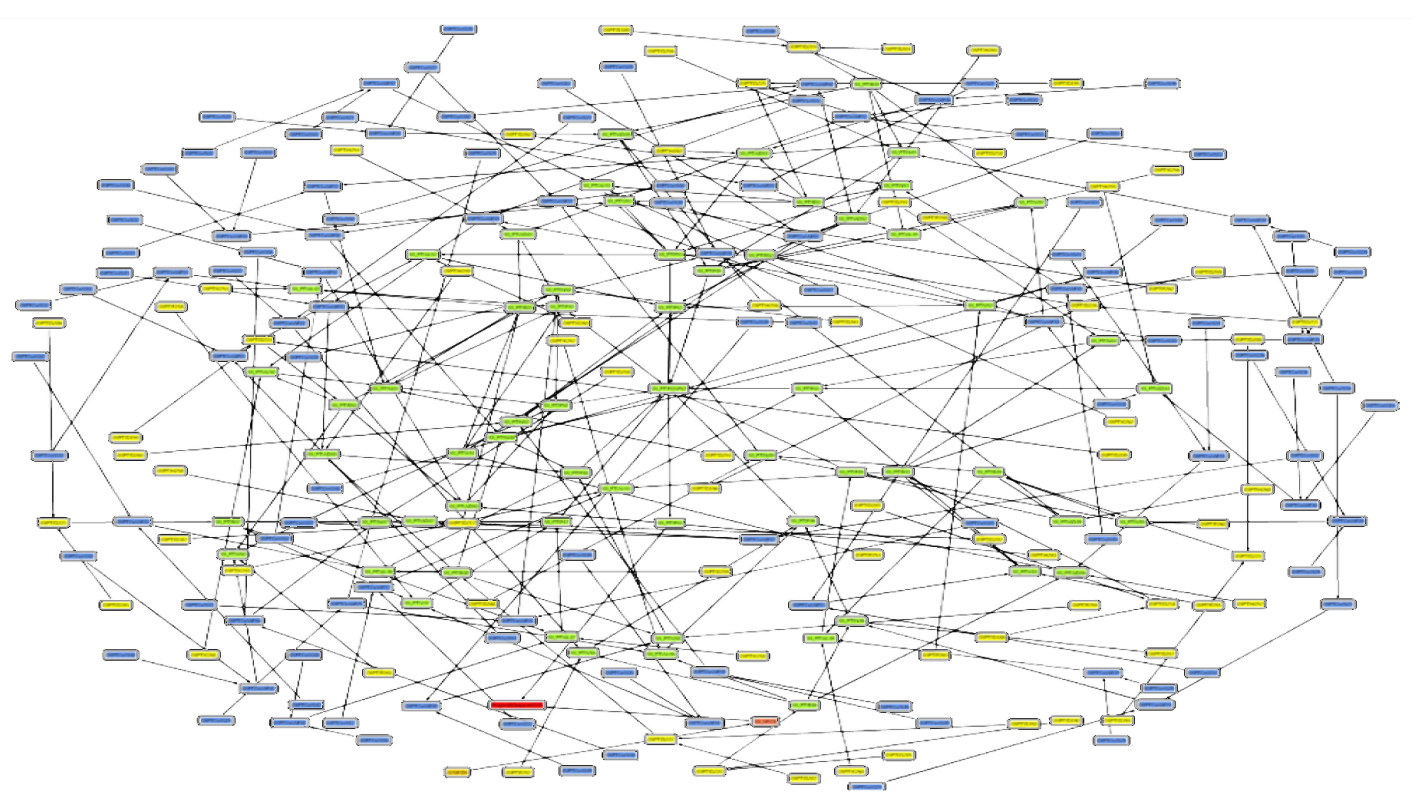

Figure 5.4: Visualisation demonstrating the complexity of the calculation graph of a small subset of Arup's IRM model. Nodes are calculations and edges show dataflow.

Having extracted a slice of interest from the calculation model we then display it interactively using a custom graphml [40] viewer. Each input, calculation step or output becomes a node in the graph with references between calculation steps and inputs 
forming the edges of the graph. Ranges (such as "A1:B3") which represent a group of cells are also shown as aggregated nodes to aid comprehension of formulas containing many ranges. Due to constraints in Excel this graph forms a directed acyclic graph. Inspired by visualisation approaches such as [90] [52] [43] we include an interactive visualisation of this graph. We are able to add extra information to the graphs by colouring each node of the graph based upon the discipline of the model it is from; showing immediately which disciplines are involved in a particular series of calculations. Additionally the graph viewer facilitates interactive exploration of the calculation graph under several layouts each of which highlights different aspects of the graph, enabling the best layout to be chosen for the calculation graph. An example graph can be seen in Fig. 5.4 (p217) highlighting the complexity of a subset of the annual percapita carbon calculation.

We now demonstrate the utility of the extraction and visualisation methods by a case study.

\subsubsection{Visualisation Case Study}

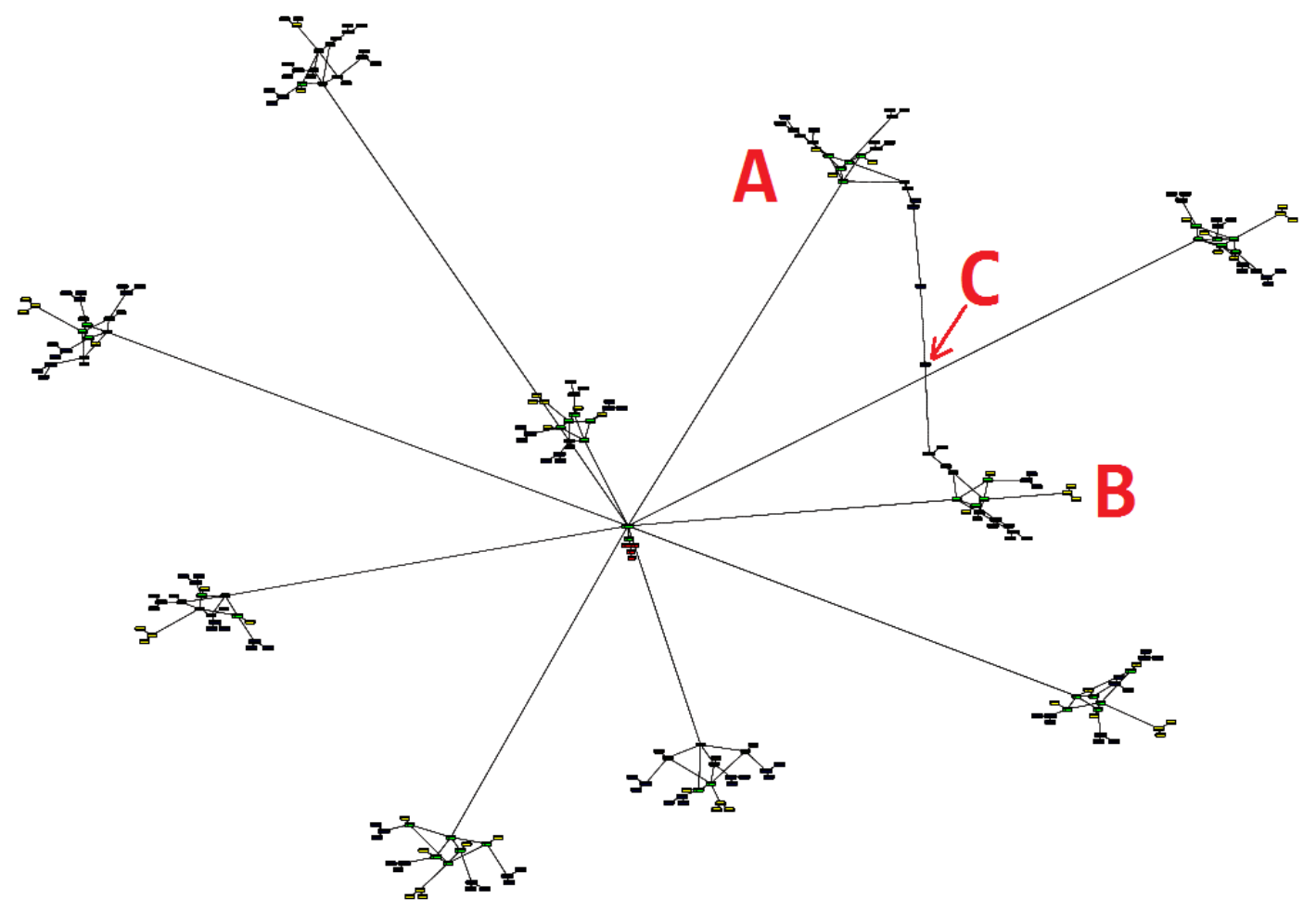

Figure 5.5: Graph showing a model slice of the calculation of the $\mathrm{CO}_{2} e$ emissions percapita per annum for external transport. Layout highlights the calculation for ten modes of transportation.

One of the sustainability metrics assessed by Arup's IRM model is $\mathrm{CO}_{2} e$ emissions 
percapita per annum for external transport. We extracted the slice of the model (255 cells) pertaining to the calculation of this metric and visualised it in our custom viewer. The graph can be seen in Fig. 5.5 (p218) laid out according to a linlog energy force model which highlights strongly connected sub-graphs. In this case we see that there are ten sub-graphs (calculations) feeding into the calculation of the metric (the central node in the graph). If this node is selected a list of the ten nodes that feed into the calculation is automatically shown and we see that each sub-graph corresponds to the calculation of carbon emissions for a different mode of transport.

An interesting and clearly visible feature of the graph is that two of the sub-calculations are connected (" $A$ " and " $B$ "). This is an anomaly since none of the other disciplines are connected and warrents further investigation. Upon selecting the node (cell " $C$ ") linking both calculation clusters (" $A$ " and " $B$ ") a list of all of the ways that this cell is used in the calculation of the metric is generated. In this case two calculation paths $(C \rightarrow A$ and $C \rightarrow B)$ are found from this connecting input cell to the final transport carbon output figure. Further investigation of these paths shows that the input value for $\mathrm{CO}_{2}$ e emissions for diesel buses per passenger kilometre is used in the calculation of both the bus and the coach mode of transport. This is unexpected since coaches are normally expected to have around a quarter of the $\mathrm{CO}_{2} e$ emissions of a bus. The effect of this will be that the carbon emissions for coaches could be overestimated in the model. This issue was reported to the IRM engineers who agreed the issue was unexpected and had been fixed in later versions of the model, but could have been an assumption carried over from a previous project where coaches and buses having similar $\mathrm{CO}_{2} e$ emissions on small islands.

This example demonstrates the utility of the slicing and visualisation tools to aid understanding and examination of a complex engineering model.

\subsection{Model Metrics}

Having extracted and visualised a slice of a multidisciplinary engineering model there are a number of graph metrics which can be automatically calculated to give insight into the calculation model. In contrast with previous approaches we consider the value of worksheet level metrics rather than formula level metrics [47] [44], particularly because of the relationship with the disciplines they represent.

Firstly we see the number of nodes in a graph and their average valency, that is the average number of cells each cell references and is referenced by. A higher average valency shows a more connected and so more complex calculation. For annual percapita carbon emissions we find the graph contains 2,357 nodes with average valency of 2.89. We can also break this down per discipline as shown in Fig. 5.6 (p220), highlighting the most complex disciplines, for example the Energy and Passenger Transport models both have 
more cells than average. We can also see models with high valency which demonstrates complexity; noticeably the project output model has average valency 14.83 . We note that a higher valency shows higher interconnectivity which makes adapting the model to meet project requirements more difficult since changes to one cell will affect many others which all must be checked for consistency if a cell is changed. However high interconnectedness may demonstrate more realistic modelling of a complex situation.

\begin{tabular}{|l|c|c|c|c|}
\hline \multicolumn{1}{|c|}{ Discipline Model } & Cell Counts & Inputs & \% Inputs & Average Valency \\
\hline Land Use (LU) & 38 & 24 & $63 \%$ & 3.24 \\
\hline Socio Economic (SE) & 38 & 23 & $61 \%$ & 1.87 \\
\hline Passenger Trans (PT) & 210 & 180 & $86 \%$ & 1.57 \\
\hline Pass Trans Coeff (PTCo) & 140 & 99 & $71 \%$ & 2.44 \\
\hline Energy Demands (ED) & 477 & 371 & $78 \%$ & 1.89 \\
\hline Logistics (Lo) & 133 & 111 & $83 \%$ & 1.33 \\
\hline Logistics Coeff (LoCo) & 16 & 16 & $100 \%$ & 2.75 \\
\hline Water (Wa) & 111 & 111 & $100 \%$ & 1.00 \\
\hline Energy Supply (ES) & 34 & 33 & $97 \%$ & 1.79 \\
\hline Energy Sup Coeff (ESCo) & 12 & 12 & $100 \%$ & 6.00 \\
\hline Convert Factors (CF) & 2 & 2 & $100 \%$ & 18.00 \\
\hline Out: Energy Dem (SSED) & 185 & 12 & $6 \%$ & 3.32 \\
\hline Out: Energy Sup (SSES) & 244 & 48 & $20 \%$ & 4.40 \\
\hline Out: Logistics (SSLo) & 67 & 0 & $0 \%$ & 3.99 \\
\hline Out: Pass Trans (SSPT) & 366 & 0 & $0 \%$ & 3.71 \\
\hline Out: Socio-Econ (SSSE) & 14 & 0 & $0 \%$ & 4.21 \\
\hline Out: Water (SSW) & 264 & 75 & $28 \%$ & 4.08 \\
\hline Project Outputs (Out) & 6 & 0 & $0 \%$ & 14.83 \\
\hline
\end{tabular}

Figure 5.6: Per discipline metrics calculated from a calculation graph extracted from a model slice for annual percapita carbon emissions. Each discipline has an input and an output model, the \% input column shows that over time some inputs have been entered in output models. Average valency is the average number of cells each cell references and is a measure of calculation complexity.

Secondly we consider the types of cells that compute each discipline's input or output model. Fig. 5.6 (p220) identifies the models with the highest number of cells. This highlights the complexity of each model and gives an indication of the data demands each discipline is likely to make. As an example we see that the Energy Demands input model contains 477 cells of which 371 are numeric inputs; thus a fair fraction of the cost of an IRM study is likely to be in fulfilling the energy demand data requirements. We note that these inputs contain both project or region specific data requirements as well as constants such as unit conversion factors. 
Finally we can see that although each discipline has both an input and an output model this demarcation is not strictly observed in all discipline's. For example we see in Fig. 5.6 (p220) that the water output model contains 75 inputs ( $28.4 \%$ of the total). On inspection this turns out to be due to summing over a range of hidden columns. Whilst not causing any errors this has the potential to cause problems should the model be adapted and those cells used for other purposes. The energy supply output model also has a large number of inputs in the output model. These are used to remove non-project specific energy sources from the model. Also we see that many input models have up to $40 \%$ non-input (i.e. calculation) cells. This is acceptable since it is summarising the input data as in land-use or converting units as in Energy Demands.

We can also consider the most referenced input data and sub-calculations. These metrics are also useful for knowing which cells should not be changed as they are used by many other discipline models. Unsurprisingly the most referenced cells are mostly conversion factors such as the $\mathrm{CO}_{2}$ e figure for NOx emissions.

Whilst these metrics give some indication of the complexity of each discipline model, it is interesting to look at the interrelationship between the disciplines.

\subsection{Discipline Coupling}

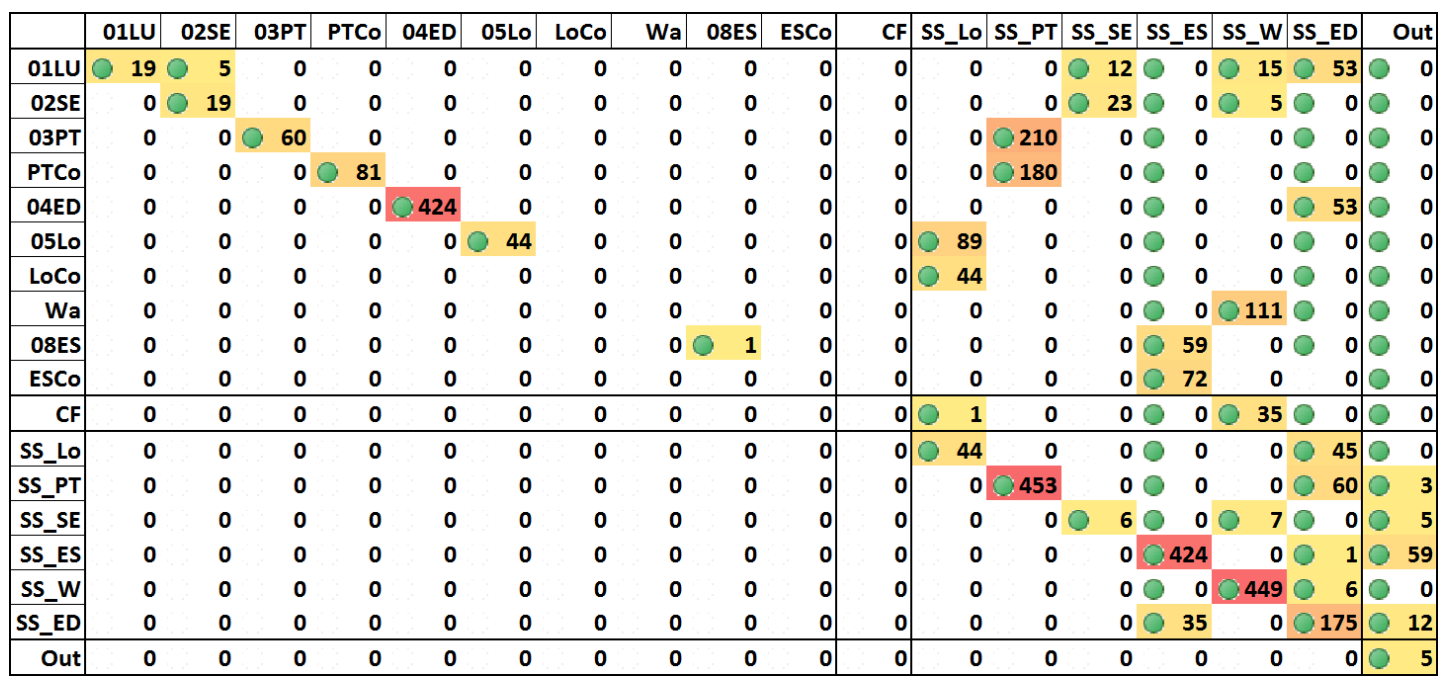

Figure 5.7: Discipline coupling matrix shows discipline communication in the IRM model. Matrix should be read " $x$ values in row model are used by column model". For example 53 values in the Land use model are used in the Energy Demand output model (SS_ED). Circular markers show indirect couplings (references made to a discipline which references another discipline).

Since the IRM model contains models from many different disciplines, it is interesting to consider the interconnections and communications between these disciplines as 
shown by data dependencies between models in different worksheets. This shows how disciplines communicate and how accurate parts of the model are by involving all concerned disciplines in, for example, the calculation of energy demand. As an example, one hypothesis proposed by the IRM engineers was that the transport model was not connected to the land-use model (since it uses software external to the spreadsheet). In order to test this, a discipline coupling matrix was created as shown in Fig. 5.7 (p221). This is calculated by considering all of the edges in the calculation graph (the references between cells) and entering them into the matrix according to which disciplines they are from/to. The first group of rows/columns are the discipline input models, followed by the conversion factors list then the various disciplines output models and the overall IRM model output model. In addition to direct references we calculate indirect references (references made via another Excel sheet) between discipline models by tracing all possible paths through the calculation graph (this is the transitive closure of the direct references graph).

From this matrix, some key features of the calculation model can be identified. As an example we see that 45 values in the output model for Logistics (SS_Lo) are used in the output model for Energy Demand (SS_ED). On the diagonal we see the number of internal references within a model which indicates the complexity of that model. We see direct communication between disciplines as indicating which disciplines are communicating by sharing results and thus where there is scope to increase collaboration and possibly model comprehensiveness. As Fig. 5.7 (p221) shows, the passenger transport and logistics models (PT, Lo and their Co-efficients) are indeed not directly connected to the land-use (LU) model, thus confirming the IRM engineers' hypothesis that there was no connection.

It is also interesting to note the number of other models (rows) that each model (column) refers to indirectly. For example, as one would expect, the overall IRM model output model indirectly references all other models, as shown in the final column of Fig 5.7 (p221). Similarly, we see that the energy demand and supply output models refer to most of the other models as is expected for a carbon calculation. This ability to detect communication between disciplines enables validation that the spreadsheet created matches the conceptual model of the IRM model as shown in Fig. 5.1 (p210).

\subsubsection{Software Metrics}

Given the number of discipline models which are composed together to form the IRM model there is much similarity between the IRM model and a large piece of software. If we consider each discipline's model as a separate code package we can apply standard software engineering code metrics such as [67] to the discipline coupling matrix.

We calculate measures of a model's responsibility to and independence from other models in terms of the data they must provide to other models and the data they 


\begin{tabular}{|l|c|c|c|}
\hline & $\begin{array}{c}\text { Afferent } \\
\text { Coupling } \\
\text { (Responsibility) }\end{array}$ & $\begin{array}{c}\text { Efferent } \\
\text { Coupling } \\
\text { (Independence) }\end{array}$ & Instability \\
\hline Land Use & $\mathbf{2}$ & 0 & $0 \%$ \\
\hline Socio Economic & $\mathbf{2}$ & $\mathbf{1}$ & $33 \%$ \\
\hline Passenger Trans & 1 & 0 & $0 \%$ \\
\hline Pass Trans Coeff & 1 & 0 & $0 \%$ \\
\hline Energy Demands & 1 & 0 & $0 \%$ \\
\hline Logistics & 1 & 0 & $0 \%$ \\
\hline Logistics Coeff & 1 & 0 & $0 \%$ \\
\hline Water & 1 & 0 & $0 \%$ \\
\hline Energy Supply & 1 & 0 & $0 \%$ \\
\hline Energy Sup Coeff & 1 & 0 & $0 \%$ \\
\hline Convert Factors & $\mathbf{2}$ & 0 & $0 \%$ \\
\hline Out: Energy Dem & $\mathbf{2}$ & 6 & $75 \%$ \\
\hline Out: Energy Sup & $\mathbf{2}$ & $\mathbf{3}$ & $60 \%$ \\
\hline Out: Logistics & 1 & $\mathbf{3}$ & $75 \%$ \\
\hline Out: Pass Trans & $\mathbf{2}$ & $\mathbf{2}$ & $50 \%$ \\
\hline Out: Socio-Econ & $\mathbf{2}$ & $\mathbf{2}$ & $50 \%$ \\
\hline Out: Water & $\mathbf{1}$ & $\mathbf{5}$ & $83 \%$ \\
\hline Project Outputs & $\mathbf{0}$ & $\mathbf{4}$ & $100 \%$ \\
\hline
\end{tabular}

Figure 5.8: Software engineering metrics normally applied to large software projects [67] are applied to multidisciplinary models to gain insight into model maintainability and stability to change. 
consume from other models. We use these to compute instability to model change so as to identify which models are most likely to cause difficulty for project adaptation.

Firstly we compute a model's afferent coupling [67] by counting the number of discipline models (worksheets) which reference cells in the given model (worksheet). This gives a measure of the responsibility of a model to other models. For example the land-use model has responsibilities to four other models (socio-economics input and output models, and output models for water and energy demand). Models with high afferent coupling are less easy to adapt to new projects as changes to the model must avoid breaking its dependants expectations.

Secondly we compute the efferent coupling [67] by counting the number of models (worksheets) which cells in a given model (worksheet) reference. This gives a measure of the independence of the model with lower scores considered more independent. For example the land-use is independent of all other models and so not affected by changes in any other models. On the other hand the Energy Demand output model is dependant on six other models and so is highly dependant. Models with poor independence are likely to be affected by changes in other models.

Finally we compute a measure of a model's instability to change [67] as follows, where $0 \%$ is stable and $100 \%$ is unstable.

$$
\frac{\text { efferent }}{\text { afferent }+ \text { efferent }}=\text { Instability }
$$

Fig. 5.8 (p223) shows the results for the IRM model. We see that most of the discipline input models are highly independent and so are not likely be to affected by changes to other models. On the other hand the output models have varying levels of dependence on other models and so have higher levels of instability. This indicates they are more likely to be affected by changes to other models.

These metrics allow engineers to discover how difficult it may be to make changes to a given model and how likely these changes are to affect other discipline's models. These results could also be taken into account when designing complex engineering models with many sub-models in order to make the model more modular and less costly to adapt.

\subsection{Formula Metrics}

Having looked at how the model works, we now look at how it is constructed in detail and how it might be improved. For this we consider the formulas which make up the model, identifying possible problems and areas for improvement. The results now discussed are from the model slice for calculating annual per-capita carbon emissions. 


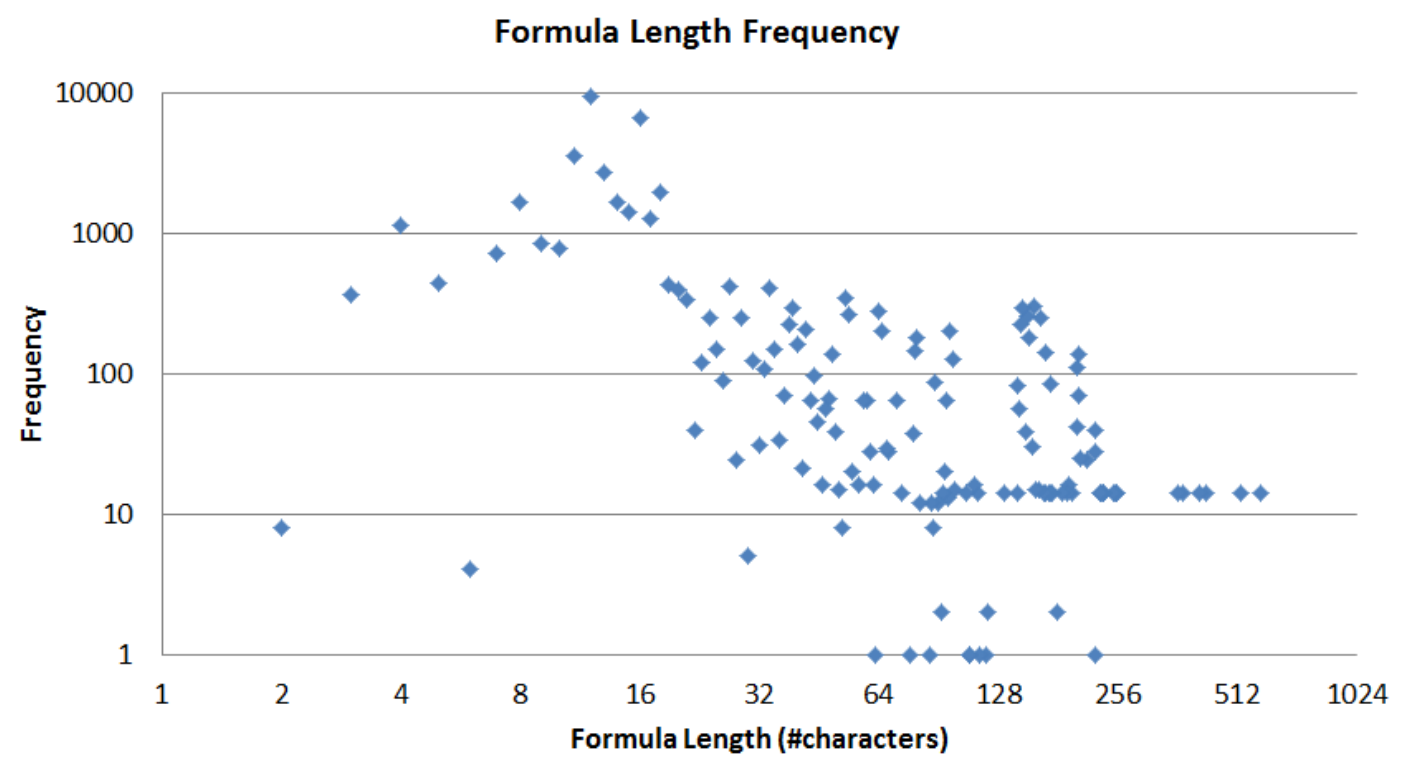

Figure 5.9: Frequency plot of the length of 50,000 formulas encountered in the IRM 2011 model. Note the large number of formulas over 100 characters which proved very difficult to maintain.

\subsubsection{Formula Length}

One very simple metric we can apply is to identify abnormally long formulas. These are difficult to understand and thus to maintain and adapt to new projects. We find the average length of formulas in the annual percapita carbon emissions model slice to be 23.4 characters. However we find that there are several exceedingly large formulas, the largest being between some 700 and 900 characters long, indeed the ninety-fifth percentile is 170 characters long. This makes formulas difficult to understand quickly. These formulas should be split into several calculations to allow easier modification and comprehension. In later models we see this has been done to some extent (Fig. 5.9 (p225)) however there still remain too many long formulas. Model checking tools such as these should be used during the development and maintenance of these models to avoid such problems.

\subsubsection{Hard Coded Constants}

One problem found when working with engineering models in Excel is that many formulas often contain undocumented numbers. In many cases their meaning can be worked out from the context for example in if (population $=0$, something, somethingelse) the 0 clearly means 0 people. However there are also many occasions where numbers or pieces of text are found in formulas and their meaning is not immediately clear. One concrete example is that of conversion factors, some of which can be quite obscure. 
Our analysis package identified that $6.5 \%$ of cells in the annual percapita carbon emissions model slice contained such hard coded constants with a total of 288 constants found. Cells with constants had an average of 1.9 constants. The constants 0,1 and "'" (empty string) account for 168 occurances, the remaining 120 constants are mainly numbers which are explainable only after detailed examination of their context. Often they turn out to be unit conversion factors which perhaps should have been entered in the conversion factors list of the model. One common constant of interest was "HEAT and/or COLD" which occured 8 times and relates to how a given energy generation technology is sized.

In contrast to the approach of [18] which searched formula strings for numbers preceded by operators, the parsing engine we employ enables the reliable identification of constants nested within functions without preceding operators and can also identify non-numeric constants such as strings.

The opacity of some of these hard coded constants prevents understanding and easy model modification since constants cannot be changed without modifying each occurrence in each formula. The solution to this problem is to extract many of the constants into new model variables (cells), giving them a clearly defined name would make their use transparent and allow easier project adaptation especially when the constant is found in multiple formulas. This commonality is also something we can investigate.

\subsubsection{Common Sub-expressions}

Repetition within an engineering model is a common source of error when modifying a model, since it is easy to omit to change one formula, especially when it is difficult to identify duplication [77]. To help resolve this problem we implemented a simple common sub-expression analysis for Excel formulas and applied it to the annual percapita carbon emissions model slice. The analysis does not take into syntactic equality and does not account for mathematical equality where $5 * x$ and $x * 5$ should be found to be identical, however 66 common sub-expressions were identified as occurring 3 or more times. An example would be "SS.ED.TransportLogisticsInternal.ElectricityDemand + SS.ED.TransportLogisticsExternal.ElectricityDemand" to obtain the total logistics electricity demand. This expression occurred in four different formulas. This example demonstrates the method of reducing duplication and formula length and aiding maintainability. Specifically a new variable called

"SS.ED.TransportLogistics.Total.ElectricityDemand" should be created and referenced such that if another source of energy was to be modelled it could be included in the total by changing one cell and not four.

In addition to these formula based metrics, it is possible to compute other model quality and complexity metrics to give an indication of the maintainability and improvements that can be made to the model. Additional metrics are discussed in literature, 
for example [20].

In conclusion we see that model slicing and model analysis give helpful insight to those maintaining and evolving complex engineering models. The metrics listed above are just a few of the possible ways of gaining insight into the model.

\begin{tabular}{|c|c|c|c|c|}
\hline \multicolumn{5}{|c|}{ Formulas Used in IRM models } \\
\hline IRM 2008 & \multicolumn{2}{|c|}{ IRM 2009} & \multicolumn{2}{|c|}{ IRM 2011} \\
\hline $\begin{array}{c}1,234 \text { Cells } \\
2,360 \text { References }\end{array}$ & \multicolumn{2}{|c|}{$\begin{array}{c}\text { 2,357 Cells } \\
\text { 3,404 References }\end{array}$} & \multicolumn{2}{|c|}{$\begin{array}{c}\text { 37,926 Cells } \\
\text { 253,222 References }\end{array}$} \\
\hline \multirow[t]{11}{*}{ SUM } & SUM & 176 & IF & 5250 \\
\hline & IF & 99 & MATCH & 2714 \\
\hline & TYPE & 81 & HLOOKUP & 2714 \\
\hline & & & ROUNDUP & 1717 \\
\hline & & & ISERROR & 1357 \\
\hline & & & SUM & 1223 \\
\hline & & & VLOOKUP & 198 \\
\hline & & & SUMIF & 78 \\
\hline & & & AND & 57 \\
\hline & & & ISNUMBER & 28 \\
\hline & & & Misc & 7 \\
\hline
\end{tabular}

Figure 5.10: We applied the EAM [61] toolkit to three different IRM models ranging from a concept model to a fully developed model to a globally used geographically localised tool. The increase in complexity is down to increased use of data tables localising the model to a region.

\subsection{Utility of Metrics}

The metrics presented thus far can be used for three main purposes. Firstly to aid model comprehension by practitioners as they learn and work with new models. Secondly to identity errors and poor quality areas of the spreadsheet based model. Finally the metrics can be used to guide model development over time. Considering each of the metrics thus presented we can identify their benefits.

\subsubsection{Aiding Comprehension}

Firstly to aid model comprehension metrics such as those presented in section 5.7 (p219) can be used to gain an overview of model complexity and its distribution amongst submodels. This is particularly apparent from the number of cells and references both in total and their distribution over sub-models as shown in Fig. 5.12 (p233). This enables identification of the most complex parts of the model in terms of the number 
of cells (e.g. Energy Demand sub-model) or in terms of connectedness as measured by the number of cell references (e.g. Project Outputs with nearly 15 references made per cell). The practitioner is then able to focus their documentation or learning efforts on the areas of greatest complexity.

Another tool aiding comprehension of the model is the discipline coupling matrix (for example see Fig 5.12 (p233)) which identifies which sub models are connected within the model. This can also be visualised as a graph for ease of comprehension. This helps practitioners to identify data flow within the model helping them to identify which models are affected by changes in others and vice-versa. This also identifies which sub-models in the spreadsheet are input models and which are the output models. This is because the slice extracted will always have a basic tree structure with many inputs feeding through calculation nodes to create a final root output of interested. This should be reflected in the spreadsheet coupling matrix. Such metrics can also be used for improving model quality.

\subsubsection{Improving Quality}

The metrics presented can be used to improve the quality of the model on three levels. Firstly improving model itself as a representation of real life. Secondly by improving the technical implementation of the model as a spreadsheet. And finally by identifying and resolving bugs in the model.

At a modelling level the discipline coupling matrix can be used to identify whether disciplines that should be connected actually are in the model. For example a connection between energy and transport is required to accurately model electric cars.

At the technical level the metrics presented can help identify quality issues in the spreadsheet. For example the percentage of inputs in some of the output models as shown in Fig. 5.6 (p220) is a concern and should be addressed. Finally the metrics presented can be used to identify bugs in the model, perhaps by identifying unexpectedly highly referenced cells or common sub-expressions which have not been updated by the maintainer. One particular source of model bugs is the identification of hard coded constants within formulas. As discussed in section 5.9 .2 (p225) these should be refactored to place common constants and assumptions in separate input cells. They present a particular issue to model quality when some but not all hard coded constants are updated leaving some formulas inconsistent.

Closely linked to improving model quality is guiding the development of the model.

\subsubsection{Guiding Development}

As will be discussed in section 5.12 (p232) repeated application of the EAM methodology and calculation of the metrics can identify changes the model over time. These 
changes in metrics can identify concerning trends in the model which should be addressed as the model is developed. For example one metric of particular concern for the ongoing development of the model is the length of formulas and the number of unmaintainably long formulas (see Fig. 5.9 (p225)).

A more complex metric for guiding the development of the model is the coupling metrics presented in section 5.8.1 (p222). Particularly an effort should be made to reduce the instability of the sub-models in the IRM model. This will reduce the likelihood that changes in one model will require changes in models which depend upon that model. This however may conflict with the requirement of faithful modelling which will tend to increase the dependencies between models (as shown in the evolution of the discipline coupling model). Finding the correct balance between these goals is a difficult task which should be approached from a software engineering perspective through the separation of concerns within models; perhaps by introducing smaller sub-discipline calculation models with responsibility for particular aspect of the model (e.g. electricity demand). These would reduce the tendency for every model to reference every other and make the flow of data and the reasons for connections more transparent.

\subsubsection{Conclusions}

In conclusion we see the varied ways these metrics can be used to benefit practitioners as their comprehend, use and maintain their complex engineering models. It is also interesting how repeated analysis of models as they develop can gain substantial insight into their evolution. This will be discussed in section 5.12 (p232). First however we consider the final part of the EAM methodology to support practitioners.

\subsection{Sensitivity Analysis}

One common engineering task is to optimise a design for a given KPI, in the case of an IRM model this might be the annual percapita carbon emissions figure. In a standard Excel engineering model this is difficult since the designer must first find all of the input cells which affect the KPI, consider their ranges and then attempt to find combinations of values which optimise the KPI whilst considering the side-effects of doing so on other KPI's and stakeholders such as the effect of introducing electric cars on the number of parking spaces in a development. Despite including a "Goal Seek" tool, Excel does not aid the user in this, since the tool still requires the user to enter a list of cells to vary. This is a difficult proposition within large engineering models since, an example, the annual percapita carbon calculation in Arup's IRM model contains over 2, 000 cells spread over many worksheets with over 900 of them being inputs that effect the output.

To aid the engineer in this process we have created tools to apply a sensitivity analysis to a slice of the model corresponding to all of the cells which are involved in 
the calculation of a specific KPI of interest. The result of a sensitivity analysis is to identify those input factors which the KPI is most sensitive to changes in. This enables the designer to focus upon only a subset of the inputs which have the most effect on the KPI, increasing their productivity.

A sensitivity analysis takes a set of factors (inputs), which affect the output of interest, along with the maximum and minimum value each factor can take. It constructs a series of model runs with varying combinations of factors set at their maximum or minimum levels with a view to analysing the results to identify the sensitivity of the output to changes in each input factor. We use a Plackett-Burman (PB) sensitivity analysis [81] [106] due to its linear relationship between the number of factors under consideration and the number of model runs required. This is key since most other sensitivity analyses are factorial in the number of runs to the number of parameters. As we have 933 separate input variables in the IRM model such factorial complexity would render the analysis intractable. However the cost of this limited number of runs is that a PB analysis cannot identify sensitivity to interactions of factors. For example the area of a rectangle is sensitive to both its width and its length but is more sensitive to the interaction of these factors namely width $*$ length. Indeed these interactions may be aliased with the main factor's effects. However a technique called folding [71] can be used to reduce this at the cost of doubling the number of runs.

\subsubsection{Case Study}

\begin{tabular}{|c|c|c|c|}
\hline \multirow[b]{2}{*}{ Variable } & \multicolumn{3}{|c|}{ Normalised Sensitivity for $\mathrm{CO}_{2}$ e Emissions Per Capita } \\
\hline & Total & $\begin{array}{c}\text { Non-Domestic } \\
\text { Buildings }\end{array}$ & External Transport \\
\hline FuelType Petrol City Car & 100 & 0 & 100 \\
\hline $\mathrm{CO} 2$ emissions from gas combustion & 91 & 100 & 0 \\
\hline FuelType Electric Heavy Rail & 78 & 0 & 78 \\
\hline District Heat Demand - Gas Boiler & 71 & 73 & 0 \\
\hline District Heat Efficiency - Gas Boiler & 71 & 73 & 0 \\
\hline Gas Network Efficiency & 68 & 83 & 0 \\
\hline Gas Network Demand & 62 & 78 & 0 \\
\hline Electricity Demand from CHP & 57 & 68 & 0 \\
\hline $\mathrm{CH} 4$ emissions from biomass & 47 & 52 & 0 \\
\hline Efficiency of Heat from biomass & 46 & 55 & 0 \\
\hline
\end{tabular}

Figure 5.11: A sensitivity analysis identifies the variable with most scope to impact a KPI. We normalise to the most impactful variable. We show impact upon total percapita carbon and side effects on some constituent parts of the total percapita carbon figure. We analysed over 900 input variables.

As an example we take the annual percapita carbon emissions KPI, extract the corresponding model slice, from which we can extract a list of all of the numeric inputs to the calculation graph. For each input the maximum and minimum range of the 
variable is established from discussions with engineers from the appropriate discipline. At the same time a meaningful name is assigned to the cell or is auto-extracted from the cell's name in Excel. Note that not all numeric inputs are actually variable, for example conversion factors should not be included and must be manually removed from the list of parameters involved in the sensitivity analysis. Once done we find 933 parameters to apply a sensitivity analysis to. This requires 2563 simulation runs, the Excel-Sensitivity application runs one run per 0.72 seconds on a Quad Core (Intel i7 720QM) machine, running four experiments concurrently. The results of the analysis are showing in Fig. 5.11 (p230). We note that we can test the sensitivity of more than one KPI to the same set of factors at very little extra cost, thus we choose other KPIs of interest such as the KPIs that show the breakdown of the total percapita $\mathrm{CO}_{2} \mathrm{e}$ emissions into its constituent parts. This shows the side implications of changes in the factors and gives us insight into the relative importance of each sub metric to the total and what scope there is for effecting each.

Fig. 5.11 (p230) shows a part of the normalised (so that the factor with most impact is 100) results of a sensitivity analysis upon the annual percapita carbon metric, with the sub-metrics for emissions from buildings and transport included in the outputs of the analysis. This inclusion of several output metrics allows us to see the side effects of changing one input to the model. For example the three fuel type metrics affect the total $\mathrm{CO}_{2}$ e emissions and the transport KPIs but do not affect the non-domestic buildings sub-metric. We can see from the results that the inputs to the energy supply (ES) discipline metrics are perhaps unsurprisingly the most important for the carbon emissions metric. Interestingly we can see that district heating has a surprisingly high effect on the carbon efficiency of the masterplan, as do Combined Heat and Power (CHP) systems should they be included in the masterplan. The full set of results includes over 900 variables though the KPI under study is shown to be highly sensitive to only the first 85 (sensitivity $10 \%$ of the maximum). These results can be broken down by discipline for more detailed insight. We see that the landuse parameters do not appear in these top 85, likely because the range of the variable being set to $\pm 5 \%$ of current design scenario does not give sufficient scope for impact. This shows that the best strategy for significantly reducing carbon emissions is either to significantly re-work the landuse schedule or if this isn't possible, to change the non-landuse design parameters identified in this sensitivity analysis. Sensitivity analysis results coupled with the implicit knowledge of discipline experts yeilds further insight into how the carbon figure may be optimised.

In conclusion we identify the following benefits of running a sensitivity analysis on an engineering model:

1. Design Insight - The designer gains knowledge of the design space and the interactions between the design parameters and the output KPIs of interest, allowing 
firstly a focusing of effort upon only those parameters KPIs are most sensitive to and secondly gaining insight into side effects of changes on other KPIs of interest should they be included in the sensitivity analysis.

2. Design Space Exploration - Whilst running the analysis we automatically create and evaluate several thousand designs. Exploration of some of these designs allows a designer to quickly understand some of the potential configurations and directions for design improvements.

3. Identification of effects of assumptions - Within most engineering models there are a large number of assumptions surrounding various factors. In the case of an IRM model we may find assumptions about the carbon emissions of buses or the watts $/ \mathrm{m}^{2}$ required for heating an office. If such assumptions are included within a sensitivity analysis (the high/low values determining the confidence interval of the assumption) the engineer gains understanding of the relative importance of the assumptions and the effects of error margins in each assumption. This suggests that time should be spent confirming and refining the most important assumptions.

\subsection{IRM Evolution}

In this section we discuss the application of the EAM toolkit to three IRM models developed over a number of years from an initial concept case study model to a fully developed globally used tool. We also demonstrate the transferability and scalability of the methodology and tools to differing models and model sizes.

Fig 5.10 (p227) shows the 3 IRM models to which we have applied the methodology. These models show the development of the IRM model over time from concept to a globally used model. Reflecting this, the size of the model has increased dramatically as more detail and accuracy have been added to the model. Partly this is since the most recent IRM model contains many data tables localised to geographical regions. The increase in size is also reflected in an increase in complexity as noted by the number of Excel functions called within the model. All figures in the table refer to the slice of the model corresponding to annual percapita carbon emissions. This increase in complexity compounds the problems discussed in section 5.2 (p211), highlighting the need for computational support.

The IRM model discussed thus far is the IRM 2009 model. We decided to look at an earlier IRM concept model to identify how the model had evolved. We were able to apply the EAM methodology to the older IRM model without difficulty and some the insights generated into the model are shown in Figs 5.12 (p233) and 5.13 (p234). It is also possible to see in detail the changes that occurred in the model between these two 


\begin{tabular}{|l|r|r|r|r|}
\hline & Cell Counts & Inputs & \% Inputs & Average Valencies \\
\hline Land Use & 17 & 17 & $100 \%$ & 2.47 \\
\hline Socio Economic & 16 & 16 & $100 \%$ & 1.06 \\
\hline Passenger Trans & 48 & 40 & $83 \%$ & 1.92 \\
\hline Pass Trans Coeff & 56 & 56 & $100 \%$ & 1.88 \\
\hline Building Energy & 48 & 48 & $100 \%$ & 1.67 \\
\hline Logistics & 85 & 77 & $91 \%$ & 1.27 \\
\hline Logistics Coeff & 72 & 72 & $100 \%$ & 1.88 \\
\hline Water & 107 & 107 & $100 \%$ & 1.04 \\
\hline Energy Supply & 9 & 9 & $100 \%$ & 8.89 \\
\hline Energy Sup Coeff & 9 & 9 & $100 \%$ & 9.67 \\
\hline Convert Factors & 2 & 2 & $100 \%$ & 15 \\
\hline Out: Logistics & 18 & 2 & $11 \%$ & 4.44 \\
\hline Out: Building En & 31 & 1 & $3 \%$ & 7.26 \\
\hline Out: Water & 164 & 0 & $0 \%$ & 5.41 \\
\hline Out: CO2 & 552 & 3 & $1 \%$ & 4.78 \\
\hline
\end{tabular}

Figure 5.12: Per discipline metrics calculated from a calculation graph extracted from a model slice for annual percapita carbon emissions from the earlier IRM concept model. Compare with Fig 5.6 (p220) to see model development.

versions spanning several years of development.

One interesting trend shown in Fig 5.10 (p227) is that of connectedness. Moving from the IRM concept to the IRM 2009 model analysed earlier we see an increase in cell count (both inputs and calculation steps). However we do not see a corresponding increase in references between cells, suggesting that many of the interdependencies had already been modelled and the model was extended to allow more detailed inputs within a discipline model. The IRM 2011 model however is dramatically more connected than the previous models, this is down to a re-implementation and more lookup data tables.

Fig 5.12 (p233) shows per discipline metrics calculated from a graph of the annual percapita carbon emissions calculation from the IRM concept model and can be compared to Fig 5.6 (p220) for the IRM 2009 model. We see that the earlier model had fewer inputs (459 vs 933). However these inputs are more tightly grouped in the input models rather than mixed in with the output models as occurs on the later model, suggesting some creep as the model evolved. We can also see that there has been a shift in the complexity of the model, from focusing on water metrics to focusing energy demand. This is a novel way of considering changes in priority in sustainability projects from project to project and overtime. This allows project mangers to ensure that the analysis team is tracking client interests by for example ensuring that the transport and energy demand disciplines are communicating so as to accurately model the uptake of electric cars. 
Fig 5.13 (p234) shows the coupling of disciplines for the concept IRM model and can be compared to Fig 5.7 (p221). We see how the disciplines and their interactions have evolved. Firstly we must note the increase in size of the model, partly due to additional output models for disciplines, and due to increased detail within disciplines. We can see an increase in computation within the input models of disciplines, along with a more even spread of computation in the output models rather than most occurring in the output $\mathrm{CO}_{2}$ e model.

As the model evolved from Fig 5.13 (p234) to Fig 5.7 (p221) we see that there has been an increase in coupling between the discipline models, perhaps to more accurately model physical realities. For example we see energy demand (ED) model is coupled directly or indirectly to nearly every other model in Fig 5.7 (p221) rather than just three models in Fig 5.13 (p234). In this way the model becomes more accurate by modelling more concepts more accurately, though the cost to this is more complexity which makes it harder to maintain and adapt to projects.

\begin{tabular}{|c|c|c|c|c|c|c|c|c|c|c|c|c|c|c|c|c|c|c|}
\hline & $01 \mathrm{LU}$ & 02SE & 03PT & 03PTCo & O4ED & 05LO & 05LoCo & 07Water & O8ES & 08ESCo & C & & SSEDES & SS_Lo & SS_I & & SS_C & \\
\hline $01 \mathrm{LU}$ & & 0 & 0 & 0 & 0 & 0 & 0 & 0 & 0 & 0 & 0 & & 0 & 24 & 00 & & 0 & 0 \\
\hline 02SE & & 0 & 0 & 0 & 0 & 0 & 0 & 0 & 0 & 0 & 0 & 0 & & 0 & 00 & & 0 & 1 \\
\hline 03PT & & 0 & 00 & 16 & 0 & 0 & 0 & 0 & 0 & 0 & 0 & 0 & & 0 & 0 & & 0 & 60 \\
\hline 03PTCo & & 0 & 0 & 0 & 0 & 0 & 0 & 0 & 0 & 0 & 0 & 0 & & 0 & 0 & & 0 & 105 \\
\hline 04ED & & 0 & 0 & 0 & 0 & 0 & 0 & 0 & 0 & 0 & 0 & & 0 & 80 & 0 & & 0 & 0 \\
\hline 05LO & & 0 & 0 & 0 & 0 & 00 & 16 & 0 & 0 & 0 & 0 & 0 & & 00 & 31 & & 0 & 45 \\
\hline 05 LoCo & & 0 & 0 & 0 & 0 & 0 & 0 & 0 & 0 & 0 & 0 & 0 & & 0 & 0 & & 0 & 135 \\
\hline 07Water & & 0 & 0 & 0 & 0 & 0 & 0 & 0 & 0 & 0 & 0 & 0 & & 0 & 00 & 111 & 0 & 0 \\
\hline O8ES & & 0 & 0 & 0 & 0 & 0 & 0 & 0 & 0 & 0 & 0 & & 0 & 80 & 0 & & 0 & 0 \\
\hline 08ESCO & & 0 & 0 & 0 & 0 & 0 & 0 & 0 & 0 & 0 & 0 & 0 & & 0 & 0 & & 0 & 87 \\
\hline CF & & 0 & 0 & 0 & 0 & 0 & 0 & 0 & 0 & 0 & 0 & 0 & & 00 & 10 & & 0 & 0 \\
\hline SS_ED_ES & & 0 & 0 & 0 & 0 & 0 & 0 & 0 & 0 & 0 & 0 & & 0 & 10 & 0 & & 0 & 21 \\
\hline SS_Lo & & 0 & 0 & 0 & 0 & 0 & 0 & 0 & 0 & 0 & 0 & 0 & & 0 & 0 & & 0 & 48 \\
\hline Ss_Water & & 0 & 0 & 0 & 0 & 0 & 0 & 0 & 0 & 0 & 0 & 0 & & 0 & 00 & 353 & 0 & \\
\hline SS_Carbon & & 0 & 0 & 0 & 0 & 0 & 0 & 0 & 0 & 0 & 0 & 0 & & 0 & 0 & 0 & & 1066 \\
\hline
\end{tabular}

Figure 5.13: Discipline coupling matrix shows discipline communication in the IRM Concept model. Matrix should be read " $x$ values in row model are used by column model". For example 24 values in the Land use model are used in the Energy Demand Supply output model (SSEDES). Circular markers show indirect couplings (references made to a discipline which references another discipline. Fig 5.7 (p221) shows how discipline couplings changed as the IRM model was evolved.

As discussed the EAM and its tools enable tracking of the changing form of engineering models giving insight to their maintainers and managers. In applying the toolkit across a range of models we have shown the transferability of the process and tools to general engineering models which are implemented as spreadsheets. Indeed we have been able to apply the process and tools to a Arup vertical transportation model from start to end within one working day reporting valuable insight into the model which was accepted by its expert maintainer. 


\subsection{Further Work}

Using the wealth of information codified in engineering models we see several extensions to this work:

Firstly we see that one preclusion to IRM adoption is the high data demands. We believe it is possible for automatic or semi-automatic methods to be applied to the calculation graph to attempt to produce an abstracted version of the model with fewer data requirements. Methods such as sensitivity analysis could be used to identify parts of the model which have limited impact upon the accuracy of the overall model results.

Secondly whilst a PB sensitivity analysis gives good insight into the model, other forms of sensitivity analysis might be applied (dependent upon their tractability). One of the more interesting would be to apply automatic differentiation tools to the overall model formula allowing accurate insight into the multi-variate sensitivities of the model.

Finally automatic differentiation would lead naturally into optimisation efforts. Given the formalisation of discipline specific implicit knowledge that has taken place to produce the variable ranges for a sensitivity analysis, it would be interesting to use these as the constraints to an optimisation engine performing constraint based optimisation upon the model. Of course such optimisation would never replace an engineer's insight into which combinations of variable values are practicable, but it may serve as an interesting decision support tool within IRM models and other engineering models.

\subsection{Conclusions}

The case study presented demonstrates the need and the value of computational tools to gain understanding of complex multidisciplinary models. The techniques explored aid practitioners in model comprehension, optimisation and evolution tasks.

Exploring the usage and construction of Arup's IRM models as a representative multidisciplinary engineering model has shown the necessity of computational tools to aid engineers, many of whom have no formal programming experience, with model comprehension, optimisation and evolution tasks. Model slicing allows reduction of model complexity to show only the salient points. Interactive exploration of the model as a calculation graph is valuable, enabling users build a mental model of how the calculation works. Model metrics are an interesting and valuable way of gaining detailed insight into the model and its composition. Metrics pertaining to the multidisciplinary nature of the model give high level insight into interdisciplinary communication. Metrics computed on model formulas aid experienced users as they seek to evolve and adapt the model to new projects. Finally sensitivity analysis is a valuable technique for understanding the relative importance of hundreds of input variables when seeking to optimise for a given KPI or to check model assumptions. Clearly there are further 
techniques which would be valuable for gaining insight into general multidisciplinary engineering models however the techniques discussed can transfer to other engineering models.

We believe the contributions of this work are to have:

1. Demonstrated the link between complex engineering models and the complex spreadsheets they are frequently implemented in.

2. Presented a tool suite for systematic, automatic analysis of large spreadsheetbased models, and a methodology for using such tools to enhance the quality and value of spreadsheet-based modelling

3. Presented static analysis tools that help identify problematic aspects of the model and diagnose defects. Demonstrated the potential for these tools by exploring three versions of Arup's IRM at different stages of development.

4. Presented a sensitivity analysis tool for large spreadsheet-based models and illustrated its value in identifying the key parameters in Arup's IRM with respect to selected performance indicators.

5. Given insight into IRM models, their evolution and construction to aid their adoption, adaptation and use in design assessment and improvement.

Having demonstrated the challenges inherent in urban masterplanning, a framework for evaluating the expedience of a variety of techniques and testing these on a live case study we have now explored a toolkit for mitigating one of the biggest challenges encountered - the debugging of complex interconnected models. We now move to draw conclusions upon our investigations. 


\section{Conclusions}

\subsection{Executive Summary}

This thesis set out to investigate the area of urban masterplanning from a computational perspective with a goal of answering the following questions:

1. What computational challenges face the urban masterplanning design and analysis cycles and particularly their intersection?

2. Which computational frameworks and techniques are necessary and effective in creating design insight in the context of urban masterplan design and analysis?

Chapter 2 (p37) reports an exploration of the computational challenges facing urban masterplanning and recent approaches to address them.

The primary research areas identified in section $2.3(\mathrm{p} 47)$ surround bridging the gap between the design and analysis cycles. This gap is not only one of different disciplines, of artistic designers and quantitative engineers and of geometry vs graphs but also a more technical and workflow related one. Recent surveys have shown [35] the average project will have only a couple of quantitative analysis cycles which last upwards of 6-8 weeks whilst there will be many more design cycles which iterate more rapidly. This mismatch precludes quantitative design improvement since design insight cannot be returned rapidly enough to affect the next design iteration. Causes for this stem from the nature and organisation of the industry but concretely have computational causes and hopefully solutions. Practically these include a lack of automation, difficulty of data management and interoperability, problems scaling models and exploring the design space amongst others.

Current literature has responded to this by focusing upon four main research areas (see chapter $2(\mathrm{p} 37))$. Firstly computational design optimisation which, whilst successful in some projects, often reduces designer freedom and has limited application (e.g. difficulty of application at concept stage). Within this field limited focus is spent on the computational platforms necessary to build such design support systems, which is a major focus of this work. Secondly, there have been attempts to apply computational frameworks used in product design to architecture with some success [36]. This 
provides some inspiration for this work. Thirdly, there has been work on complex integrated, often proprietary design analysis tools which use closely integrated analyses to assess a building's design performance [49] [10]. Whilst effective these are rarely sufficiently open or scalable enough for use on a masterplanning project. Finally, there are numerous projects aiming to bridge the interoperability gap between design and analysis software through shared industry formats and more recently Building Information Management (BIM) models. However these research fronts still leave open the question of which computational techniques are most appropriate and effective in aiding the design/analysis cycle. This is the gap this thesis investigates.

Based upon these findings a set of computational hypotheses for data models and techniques thought to be effective in producing design insight on the urban masterplanning scale were set out and these were then adapted and unifying into a computational framework developed in Chapter 3 (p85).

The Hierarchical Synthesis or HierSynth framework was developed with the goal of bridging the gap between the design and analysis cycles. HierSynth aims to query design files creating a hierarchical decomposition of the design, then to use industry standard analysis models to automatically analyse the design at various scales under numerous analysis scenarios (e.g. flooding, drought...). HierSynth's goal is then to be a test bed for using various computational techniques to synthesis insight into the design from analysis data. This is done via a suite of techniques ranging from scenario generation and composition, sensitivity analysis through to an integrated $3 \mathrm{~d}$ visualisation environment for design and analysis data.

The key contribution of this thesis is the reconceptualization of the workflow graph by composing it with tree based design decompositions more commonly found in industry interoperability file formats. This is achieved through a hierarchy of design queries, templates and analysis which, when combined through a novel dataflow algorithm form, when executed, a design hierarchy annotated with analyses to be performed. This enables simple multi-scale analysis at all levels of detail, a key feature for use in the realm of urban masterplanning. This data structure is then reconceptualised to provide scenario generation capabilities and used sequentially allow the specification of design space exploration experiments to answer the "what if" questions frequently asked by engineering teams. This framework combined with techniques including sensitivity analysis, performance trees at every level and integrated 3d visualisation, are applied to the discipline of urban masterplanning to bridge the growing computational gulf between architectural design and analysis teams.

This framework was then used as the basis for exploring the second research question via a case study application of the framework and its techniques to a live masterplanning project with Arup North America. This is discussed in depth in Chapter 4 (p139) which explores the way the computational techniques and data models were applied to the 
project's challenges and were used to create design insight.

We found three stages of application providing three levels of value to the project. Firstly simple workflow automation provided substantial time savings together with faster and more frequent quantitative design insight. Secondly, we used model integration and scenario composition to move beyond current best practice by creating a highly complex integrated model ensemble (see section 4.9 (p157)) which provided detailed scenario and design comparison under many Key Performance Indicators (KPI's). Finally, we used the computational platform as the basis for undertaking designer lead investigations into the design space for the masterplan and its sustainability strategies. In this phase we found the unification of computational techniques into a common platform to be critical, particularly the analysis of a hierarchically decomposed design using the automation of an integrated model ensemble to generate insight under numerous generated and composed scenarios. This enabled us to explore the design space using techniques such as sensitivity analysis for generating insight.

A critical discussion of the performance of these techniques is provided in section 4.15 (p199), together with an exploration of the potential of further computational techniques. One of the major unexpected requirements identified during work with Arup is the need for tools to comprehend and quality check complex engineering assessment models based in spreadsheets which very frequent and surprisingly complex.

In Chapter 5 (p209) we explore a suite of tools for systematic, automatic analysis of large spreadsheet-based models, and a methodology for using such tools to enhance the quality and value of spreadsheet-based modelling. We applied this framework and methodology to Arup's Integrated Resource Management models [12] [75] and explored the insight we generated in this way. Particularly of interest was assessing the model's evolution from a "simple" 1,000 cell model through to a world wide 50,000 cell model and identifying its change in focus from water sustainability to energy demand and passenger transport.

Together I believe these threads of work weave together to contend for the thesis underlying this work, namely that:

The key to generating quantitative insight for practitioners within urban masterplanning is the unification of computational techniques such as analysis automation, model integration, scenario composition and sensitivity analysis in a single framework to provide multi-scale insight by enabling design space exploration and detailed design performance metrics.

Particularly, chapter 2 (p37) shows the need for such solutions within urban masterplanning, chapter 3 (p85) describes such a framework providing this unification of techniques which were then successfully applied to a live masterplanning project in 
chapter 4 (p139) with varying levels of effectiveness (section 4.15 (p199)) to provide improvements to designer/engineer workflow and many practical insights into the design cycle.

We will now discuss the strengths and weakness of the evidence presented for this thesis in more depth together with avenues for further work.

\subsection{Critical Analysis}

This section evaluates a number of aspects and perspectives on the evidence presented within this document to support the thesis set out above. We will explore the transferability of the lessons learned through our experimental approach. We consider the performance of the HierSynth framework, particularly as applied to the case study presented in Chapter 4 (p139). We explore this work from the perspective of designers, upon the building scale and as compared to the literature on computational design optimisation.

\subsubsection{Approach}

This thesis has explored the computational challenges within urban masterplanning and presented a computational framework to act as a test-bed for exploring the efficacy of techniques to address these challenges. Whilst there is a breadth of literature covering a number of fronts there is limited work exploring the efficacy of computational techniques to bridge the design / analysis gap which reduces the comparability of this approach. The development of the HierSynth framework enables the testing of several computational techniques, however it only allows the exploration of one data model (as described in section 3.6 (p96)). This data model is central to the unification of the techniques employed however it is likely alternative data models may perform well also, although this is not something we are able to test. The case study application to a project whilst creating much insight for the project team shows the potential value and effectiveness of the unification of computational techniques into a computational framework. These lessons should be transferable to similar projects since they will have comparable workflow and analysis requirements; although with different models and data. The breadth of analysis driven investigations undertaken gives some strength to the thesis that such a computational platform is key to deriving quantitative insight into the design space. Such investigations would not have been possible without the unification of the computational techniques found within HierSynth. 


\subsubsection{HierSynth}

A critical discussion of the performance of the computational techniques HierSynth employs is provided in section 4.15 (p199). Here we discuss the usability and performance of the HierSynth framework.

\section{Utility}

In terms of usability HierSynth remains an expert tool and was only used by the author. This is a limitation due to the complexity of interface for setting up the model integrations. Making a computational framework simple enough for use by practitioners is a challenge but one that should be addressed to gain wider use. Inspiration might be gleaned from the rise of complex parametric modelling systems in a graph based environment [41]. More widespread use of such a framework should give greater evidence for this thesis as a computational platform is more deeply unified into the design and analysis process and their workflow.

The HierSynth framework was found to be stable, had good performance characteristics (once caching and parallelism were implemented) and was generally flexible enough for day to day use without code modifications, particularly during the investigations in section 4.12 (p171). That said the framework and its data model did develop and change through the case study, most notably with the introduction of execution chains (section 3.10 (p114)) which enabled the re-use and composition of multiple scenario generation and analysis trees.

The HierSynth platform was well able to manage the execution of over 6,000 analyses linked together and generated from a pair of coupled scenario generation trees. The parallel analysis framework enabled upto 5 spreadsheet based analyses to be run concurrently and the caching framework was heavily used to avoid constantly querying static lists of configuration data. Most investigations run by HierSynth were run within 15 minutes with the longest taking around 6 hours (all with no manual input).

One limitation with HierSynth was a lack of data interrogation tools and often post processing had to be done in spreadsheet form. This was mostly due to the number of dimensions of the data (building, district, design, construction phase, scenario and so forth), however tools should be included to deal with these natively.

Two features which are sorely needed in a framework such as HierSynth is better version and change management together with a collaborative interface to enable practitioners communicate and work together to undertake a design space investigation using correctly configured and integrated analysis models. 


\section{Validity of Results}

A common question on HierSynth surrounds the validity of the analysis results. The models integrated into the HierSynth framework are generally mature widely used and industry standard models. Hence we are left with two questions.

1. Have the models been validated to ensure correct real-life results?

2. Is HierSynth using the models correctly to ensure validity of results?

The answer to the first model is normally "yes" and several of the models employed have grown from research programs (e.g. Radiance [103]). The models employed are sufficiently "best practice" and widely used in industry to be the best in class. In either case validation concerns are with the models integrated and not the computational platform. The second question is more challenging. Once another level of abstraction (HierSynth) is added to a complex engineering model it is difficult to know the correct ways in which a model be used and its results interpreted. (This is apart from ensuring that HierSynth does not mangle the results of the analysis, which is addressed through software testing). This second challenge presented a number of issues during the case study presented (see section 4.9.2 (p161)) since Arup's models were being used though automation in more situations than typical. Particularly it became easy to make assumptions about the model without validating the model support use in this way. How to avoid such model mis-use is an area for further research.

\subsubsection{Data Model}

The hierarchical data model discussed in section 3.6 (p96) worked well throughout the project. It adapted to project demands allowing analysis by development, district and building levels as required. The query-analyse-evaluate execution pattern (see section 3.7 (p100)) was effective. However the data layers and performance trees were not as useful as expected, this was mainly due to difficulty in knowing ahead of time which KPI's would be of interest and then meaningfully creating functions to aggregate disparate performance scores. That said they were used effectively in the block level $3 \mathrm{~d}$ visualisation environment to effectively display quantitative insight (see Fig. 4.16 (p170)).

The generation and composition of scenarios using HierSynth trees with different execution semantics was critical to enabling the investigations discussed in section 4.12 (p171) this together with execution chains enabling the re-use of analysis set-ups made exploring new parts of the design space quick to set up and proved a flexible and efficient data model. 


\subsubsection{Case Study}

The case study application of the HierSynth framework to a live Arup project provided valuable insight and experience enabling the testing of numerous computational techniques and identifying further requirements. The techniques the HierSynth platform unifies were able to create many insights for the analysis team. These were created in three phases: from automating current practice, to improving it and then using the platform as a basis for design space exploration.

This final phase of the case study provides perhaps the best evidence for the thesis presented. We found that a platform enabling exploration of the design space in response to questions posed by the team provided many insights of value to the team (see top ten findings 4.14 .2 (p198)). Key to this was the generation and composition of scenarios (for example different uptakes of electric vehicles with different carbon scenarios and designs) following by automated analysis using an integrated model suite. Sensitivity analysis was also a key technique in providing value (see section 4.13 (p187)). This unification was the critical enabler in this work.

To back up the thesis, the computational techniques unified must serve to bring together the design and analysis communities producing insight for both. We will discuss the impact upon both communities during this case study.

\subsubsection{Engineering Assessment}

Much of the case study in chapter 4 (p139) is primarily targeted at the engineering analysis carried out on the masterplan. Indeed, the majority of the insight generated whilst of use to the design team was generated for the analysis team. Section 4.15 (p199) discusses which techniques were most successful in generating quantitative design insight and design space exploration. The HierSynth platform was able to substantially reduce the time taken for an analysis iteration by automating model runs and was able to provide over 200 times as much data as previous available identifying new insights. For example, comparison of design performance over different construction phases and carbon scenarios similarly gave more detailed insight and allowed the exploration of trends and possible futures. These included identifying model bugs which affected only one district under one construction phase - a bug which would have been completely hidden previously. The integration of models together gave more accurate analysis and new capabilities which were previously not available to the engineering practitioners. Finally, sensitivity analysis and design space exploration techniques were used to interrogate the model and design performance allowing identification of optimal design points and the side effects of various strategies upon the development. 


\subsubsection{Designer Interaction}

This investigation has focused primarily on aiding the engineering analysis side of the AEC industry, perhaps at the expense of the design side. Practically this has been driven by working with an engineering consultancy and an analysis team on a masterplanning project. By this we mean that, whilst the results and investigations undertaking during the case study are directly useful to the design team, the case study does not consider direct impact upon geometrical form, building layout or architectural style. Similarly whilst this work has shown the efficacy of a number of computational techniques in providing insight to engineering practitioners it has only had limited success in demonstrating the value of these techniques to designers; in so much as they are not applied to the geometrical forms or analysis which will directly affect the architects shaping of design form. The solutions to these challenges are closer integration with designers, their design tools (CAD programs) and geometrical analyses. This lack of integration is a limitation of this work. However during the case study all results were fed back to the design team and were well received. Particularly the visualisation system proved a key technique for sharing analysis results, showing design performance and generating insight for practitioners. Particularly it has the scope to improve communication between the design and analysis cycles. More direct communication could be achieved by addressing:

- Closer integration with designers and their CAD software.

- Inclusion of more geometric analyses which will have concrete effects on the form of a building or development.

- Working more closely with the designers to provide a quicker design - analyse visualise loop.

- Enabling the computational exploration, and possibly optimisation of designs encoded as parametric or procedural models.

The visualisation completed in section 4.11 (p166) shows the potential for quantitative analysis of design form. This should be developed further by analysing multiple geometry and enabling the designer to explore the design space in terms of design options and performance under different analysis scenarios with different KPI's enabling the exploration of trade offs and spurring innovation.

\subsubsection{Computational Design Optimisation}

Computational Design Optimisation (CDO) involves linking a parametric or procedural design tool to an analysis model via a genetic (or similar) optimisation algorithm with 
a goal of finding the best form under one or more analysis criteria. This is a widely used technique in the literature [102] and increasingly in practice [89]. One limitation of the approach presented in this thesis is that it does not undertake morphing of the form of the design in contrast to other research. However this research has shown the potential of other computational techniques such as sensitivity analysis to provide value to the urban masterplanning community, some of whom are cautious to adopt CDO principles. Hence I believe that whilst CDO is a useful technique it is just one technique among many for providing insight to designers. It is popular because it involves direct morphing of the form of a building. As we have discussed this is something not currently possible in the HierSynth framework and should be addressed in future work.

\subsection{Thesis Conclusions}

The thesis that underpins this work was set out to be:

This thesis contends that the key to generating quantitative insight for practitioners within urban masterplanning is the unification of computational techniques such as analysis automation, model integration, scenario composition and sensitivity analysis in a single framework to provide multi-scale insight by enabling design space exploration and detailed design performance metrics.

Based upon the work set forth in this document we believe the following conclusions are valid:

- There is substantial potential for a computational platform to improve the integration and speed of the design and analysis cycles. (see section 4.7 (p151)).

- Model integration is challenging, particularly with spreadsheet based models (see section 4.9.2 (p161)), there is substantial need and potential for computational tools for investigating and resolving model challenges (see Chapter 5 (p209)).

- Model automation, integration and scenario generation provides new capabilities and more detail that provides practitioners with new insights allowing design and model improvement. (see section 4.10 (p162)).

- Far from removing the need for engineers, the application of a computational platform required as much if not more of their time and insight. By automating unnecessary manual work and providing substantially more data greater a need was created for expert interpretation and insight. 
- Hierarchical decomposition and analysis of a design provides greater insight which is essential to understanding how a design may be improved. (see Fig. 4.7 (p156) and section 4.8 (p153)).

- Scenario generation and composition together provide an effective means of design space exploration lead by designer intuition. (see section 4.12 (p171)).

- Automatic quantitative $3 \mathrm{~d}$ visualisation is an effective means of communication and design (space) exploration between the design and analysis communities. (see section $4.11(\mathrm{p} 166))$.

- The unification of computational techniques provides a firm base for design space exploration (see section 4.12 (p171)) enabling many insights into design options (see section $4.14 .2(\mathrm{p} 198))$.

- Sensitivity analysis both of models and of designs has the potential to greatly aid the design process for urban masterplanning and analysis. (see section 4.13 (p187)).

- To fully benefit from the unification of computational techniques close integration with the design team and their design tools is required. 


\subsection{Contributions}

In conclusion we claim the following contributions resulting from this investigation:

1. We present a reconceptualization of a workflow graph by composing it with design decompositions more commonly found within architectural design formats. This is achieved through a tree based specification of design queries, templates and analysis nodes combined through a novel dataflow algorithm. This enables detailed insight and multi-scale analysis at every level. Such trees are then reconceptualised to generate scenarios for design space exploration. These features combine to address many of the computational needs of the urban masterplanning community and are implemented in the HierSynth framework.

2. We present a novel computational framework (HierSynth) that supports the composition of design queries, analysis models and so-called 'performance trees' together into a hierarchical model that supports design decomposition, scenario generation and composition, workflow automation, model integration, sensitivity analysis and user-driven design space exploration. The framework provides a foundation for exploring the efficacy of computational techniques in unifying urban masterplanning and producing design insight.

3. We present a detailed evaluation of the HierSynth framework and its data model on a major commercial urban masterplanning project, in conjunction with Arup North America. This enabled exploration of the benefits of computationally unifying urban masterplanning and evaluation of the efficacy of the computational technique employed. This proved the framework can enable an order of magnitude more analysis cycles than normally feasible. The framework enabled investigations such as identifying the optimal mix of residential and commercial development for district energy system performance and reduced transportation carbon emissions. The case study revealed requirements for adopting version control and provenance tracking for design files and analysis models and, in particular, aids for investigating and debugging complex spreadsheets which form the basis of many analysis models.

4. We present a general tool suite that supports the systematic, automatic analysis of large spreadsheet-based models, and a methodology for using such tools to enhance the model's quality and value. Large spreadsheets are used in many disciplines, but are particularly important here as they are often the tool of choice in the analysis of urban masterplanning projects. The tool suite is evaluated by exploring three generations of Arup's Integrated Resource Management (IRM) Models at different stages of development, but the potential applications of the tools and associated methodology are much more general. 


\subsection{Further Work}

From this investigation and to provide further evidence for this thesis a number of additional computational techniques should be explored and applied to aid the architectural design and analysis communities. In addition to these there are a number of areas which may also benefit from a similar application and adaptation of computational techniques into a unified framework to aid practitioners.

\subsubsection{Development of Techniques}

The application of the HierSynth framework demonstrated the efficacy of a number of computational techniques on a live masterplanning project. There are a number of additional techniques which should be developed and adapted for use in this setting. Many of these are finding from the case study application of HierSynth and are discussed in length in section 4.15 (p199).

- Integration with other Disciplines - The more plugins provided on a system such as HierSynth the more valuable it will be, in part due to the ability to integrate models together. Candidates include wind flow and pedestrian modelling.

- Integration with CAD and Parametric Models - To enable improved discussion with architects it is necessary to explore the impact of changes in form. This is best done by integrating a computational platform tightly with the design tools currently in use.

- Machine Learning - Given the volume of data produced from analysing each design element (building, street, city) new techniques are required to identify trends.

- Optimisation Algorithms - These would be useful for exploring specific design challenges which can be formulated specifically enough for exploration.

- Improved Debugging and Error handling - Given the complexity of the modelling and analysis undertaken it is important that good means of investigating unexpected results are in place.

- Analysis unit testing - Given the fluid nature of some spreadsheet models employed we found that the automation process acted as a regression test for the model which some model changes broke. This highlighted the potential and need for a unit testing framework for such models, particularly given their complexity.

- Version Control - As designs and models evolve it is critical to ensure this change is managed correctly and all practitioners share a common up to date version of documents. 
- Collaborative Environment - Would enable real time collaboration on the set up of analysis and design space exploration experiments. Similarly collaborative investigation of results would be useful.

- Cloud Computing - Enabling substantial increases in analysis detail and design space exploration.

- Immediacy of feedback - Allowing results to be fed back whilst interactively sculpting the design.

- Sensitivity Analysis - Should be developed further to investigate more detailed interactions of groups of parameters together.

- Report Generation - Much of the analyst's time is spent preparing figures for reports. Automating this production would be valuable.

- Data Provenance - Tracking of which analysis results were calculated with which models from which data under which scenarios and who was involved is critical information and recording a provenance trail would be highly useful.

- Industry Data Formats - Integration with common industry data formats such as Collada, GBXML, IFC and CityGML would prove valuable. Some of these have a hierarchical layout and would map well to the HierSynth platform.

- Analysis Library - A set of standard configurations for analysis models which enable the simple adoption of an analysis for a project.

Together with these specific improvements, a number of new research directions could be addressed in future work.

\subsubsection{New Directions}

Here we discuss a number of possible future directions and new areas which could benefit from similar techniques and approaches to those discussed in this thesis. With the aim of aiding the process of generating insight into the architectural design and analysis community.

\section{Interoperability Formats}

As discussed in section 2.8 (p71) there are a growing number of industry standard interoperability formats such as IFC [22], CityGML [59], GBXML [37] and Collada [55]. Creating a platform which can read from many of these formats would improve the utility of a computational platform and help further bridge the gap between the design and analysis disciplines. There have been a number of attempts to create query 
languages for BIM models [70] [19] these could be integrated into HierSynth directly as query nodes. Perhaps in conjunction with recent work on generating model views [19]. into HierSynth directly as query nodes, particularly with recent work on generating model views [46]. Several of these formats are hierarchical in nature and should prove a good fit with HierSynth and should benefit from multi-scale analysis enable by the HierSynth data model.

\section{Building Scale \& BIM models}

This thesis has focused upon the urban masterplanning scale of design, drawing inspiration from the successful application of computation to product design. Between these scales lies the field of building design. From discussions with practitioners similar challenges to those facing urban masterplanning design and analysis also apply upon the building scale. Hence there is a fascinating area of research to be undertaken in applying similar computational unification to the building scale. Whilst there is nothing within the HierSynth framework that is specific to urban masterplanning much work would be required to create the correct set of plugins to the framework to enable reading of complex building designs from Building Information Management (BIM) models [31] and to run the correct analysis models. There are however several research projects looking to create query languages for BIM models [19] [70] enabling the extraction of model "views" [46] that support a particular practitioner in a task (e.g. showing only the ventilation system of the building). These research areas should map easily into HierSynth's hierarchical query framework since most BIM models are based in hierarchical XML [22]. This is an exciting area of future research, foreseeable challenges include an increased level of detail and interrelation between disciplines, greater interoperability challenges which are already an area of research and a difficulty of model validation which is already a known challenge in the industry [64].

\section{Collaboration \& Provenance}

Giver the interdisciplinary nature of the design and analysis communities which bridges not only disciplines but different firms. It is necessary to provide a collaborative environment for analysis work to be undertaken. This may be as simple as a firm providing an analysis service to as detailed as a team of analysts setting up a complex integrated model ensemble. Key research questions in this area remain enabling collaboration whilst maintaining confidentiality, version control, tracking of chargeable work and the provenance [72] of all design model changes and analysis results. Some BIM environments are beginning to move toward this direction [4] however a wider more open framework providing automation and design space exploration has potential. 


\section{Parametric Design \& Computational Design Optimisation}

Parametric modelling creates a design which can reparameterised to create variations on form. This capability is increasingly compounding the gap between the design and analysis communities by creating more design options in a shorter time frame. This is a driver for the adoption of analysis platforms such as HierSynth and work should be undertaken to integrate with such design tools. These tools are now being used together with optimisation algorithms and whilst it is the author's opinion that computational optimisation will never get to a point where it can replace an architect this technique is generating substantial insight and value in specific cases. Optimisation should be integrated into the HierSynth platform to enable exploration of the design space using the analyses integrated into the platform. This should enable exploration of the design space using geometrical aspects of the design which enables better communication with architects and stakeholders.

\section{Participatory design}

Participatory design is a movement to involve the client and other stakeholders more directly in the design process [95]. This involves real time exploration of design ideas and analysis of their consequences. This approach has been successfully in a decision theatre environment [76] for city planning at a high level. A room with five projects is backed by a compute cluster enabling rapid investigation of design options and scenarios. A similar approach would be effective on the urban masterplanning and building scale if geometry generation and analysis is sufficiently rapid. A stepping stone to this is precomputing a large number of design options under many scenarios and packaging the results as an explorable $3 \mathrm{~d}$ environment, similar to the prototype in section 4.11 (p166). Challenges in this area include improving analysis speed and enabling rapid changes in geometry and scenarios. Defaulting of analyses is likely to be a critical enabler, enabling detailed analyses on only high level design data by making reasonable assumptions about the internals of a building or the materials in use.

\section{Sensor Data, Smart Cities \& Operational Management}

The computational insight we have generated during the design process may be extendable to the operational phase of a building's life. Integration with city infrastructure, sensors and building management systems together with existing facilities management data stores should provide a rich seam of information which when combined with data analytics and expertise in modelling should provide valuable insight.

Research in this area is needed to manage vast quantities of data, to mine data streams correctly. Critical to success in this area is, I believe, integration with advanced models. For example integrating control of a buildings heating system with a thermal 
model of the building should enable substantial optimisation of power consumption and identify areas in need of retrofitting. This may also bring challenges with the validation of the models in current use by the industry [64]. Integration in real time with resource usage patterns may also provide novel business models particularly when combined with social media.

\section{Possible Futures}

From discussions with practitioners and from our experiences of exploring possible future scenarios using the HierSynth platform (see section 4.8 (p153)) there is a clear need for tools to enable practitioners and leaders to explore future scenarios. A key driver for this is modelling adaptation and mitigation strategies for avoiding climate change. For example the adoption of one technology now may preclude the adoption of a more efficient future technology or conversely without taking action now future sustainability strategies may become impossible. A simple example might be the planting of trees in a urban area which if done now will in the future reduce air pollution, flooding and some of the urban heat island effect. Without this strategy substantial energy demand will be created by air conditioning plants.

Adaptation of the HierSynth data model should be investigated to enable cities to explore potential strategies and external events, measure their effects and side effects and explore interactions in possible future scenarios. This would require scenario generation and composition, model data mining (e.g. sensitivity analysis), deep integration of models across disciplines. There is also the potential to add optimisation and design space exploration algorithms to identify key decisions and impacts which enable further strategies or avoid unwanted consequences.

\section{Future Research Challenges}

From these research areas a number of research challenges arise include:

- Increasing computation within existing business practices and workflow - How can computation aid the workflow between increasingly complex computational design tools and similarly complex engineering analyses? Collaborative working environments, version control and change management are key challenges. How does the structure of the industry require changes in computational platforms and market places?

- Data Analytics - Given the scope of computational support to generate more detailed data across a wider portion of the design space it will become increasingly critical to have good data processing and analytic capabilities, how recent advances apply to the AEC industry is an interesting proposition. 
- Interoperability - this is and will remain a key research challenges and a wide body of literature surrounding this area is growing. More studies should be undertaken on how this research could be practically implemented in design and engineering consultancies.

- Model Integration - There is much scope for insight arising from the integration of models, however this is a non-trivial challenge and should be investigated further.

- BIM model interaction with computational analysis - How can a computational analysis platform such as HierSynth interact closely with BIM models, BIM query frameworks and analyses?

- Sensitivity analysis in geometrical form - Sensitivity analysis has proved a successful techniques for architectural analysis and is also used for some aspects of product design. How can sensitivity analysis be applied to modification of the geometric form of a building or urban masterplan? Which forms of sensitivity analysis will be robust enough to give meaningful insight? Which architectural analyses will enable assessment of performance?

Computation continues to facilitate great advances in the AEC industry, the future will be a more open, widely used, connected and unified approach to computation across disciplines and companies. This is a future I look forward to exploring. 



\section{Bibliography}

[1] AmazonEC2. Amazon elastic compute cloud. Website, September 2012. http: / / aws.amazon.com/ec2.

[2] Arup. Comparing IFC and designlink. Website, May 2010. http:// arupforge.arup.com/wiki/index.php?title=DesignLink_and_IFC.

[3] Arup. Design link sdk wiki on arup forge. Website, May 2010. http: //arupforge.arup.com/wiki/index.php?title=DesignLink_SDK.

[4] ASITE. Adoddle - document management system. Website, November 2012. http://www.asite.com/adoddle/aec/ aec-building-information-modelling/.

[5] Autodesk. Autodesk autocad. Website, November 2012. http://www . autodesk.co.uk/adsk/servlet/pc/index?siteID=452932\&id= 14529689 .

[6] Autodesk. Autodesk ecotect analysis. Website, November 2012. http://usa . autodesk.com/ecotect-analysis/.

[7] Autodesk. Autodesk ecotect analysis product documentation. Website, November 2012. http://images.autodesk.com/adsk/files/ autodesk_ecotect_analysis_2011_brochure.pdf.

[8] Autodesk. Autodesk green building studio. Website, November 2012. http: //usa.autodesk.com/green-building-studio/.

[9] Autodesk. Autodesk green building studio product documentation. Website, November 2012. http://images.autodesk.com/adsk/files/GBS_FAQ_ 6_7_11.pdf.

[10] Autodesk. Autodesk lab's project vasari. Website, November 2012. http: //labs.autodesk.com/utilities/vasari/.

[11] Autodesk. Autodesk revit. Website, November 2012. http://www . autodesk . co.uk/adsk/servlet/pc/index?siteID=452932\&id=14645193. 
[12] E. Ayaz and J. Levitas. Spatially linked integrated resource management (IRM): A tool to inform eco-city planning. Proceedings of the 8th International Eco-city Conference, 2008.

[13] BiMserver. Bimserver - open source building information modelling. Website, November 2012. http://bimserver.org/.

[14] D. Birch. Hiersynth - automating city-wide architectural analysis for design insight. Arup Doctoral College Conference, 2011.

[15] D. Birch. Computational investigations in masterplan design. Arup Doctoral College Conference, 2012.

[16] D. Birch, P. Kelly, A. Field, and A. Simondetti. Computationally unifying urban masterplanning. ACM Computing Frontiers 2013, Ischia, Italy, 2013.

[17] M. S. Bittermann. Intelligent Design Objects (IDO) - A cognitive approach for performance-based design. Delft University of Technology, 2009.

[18] P. J. Blayney. An investigation of the incidence and effect of spreadsheet errors caused by the hard coding of input data values into formulas. Proc. European Spreadsheet Risks Int. Grp, 2006.

[19] A. Borrmann and E. Rank. Specification and implementation of directional operators in a 3d spatial query language for building information models. Advanced Engineering Informatics, 23(1):32-44, 2009.

[20] A. Bregar. Complexity metrics for spreadsheet models. Proc. European Spreadsheet Risks Int. Grp, pages pp.85-93, 2004.

[21] buildingSMART Alliance. The building smart alliance. Website, October 2012. http://www.buildingsmartalliance.org/.

[22] buildingSMART Alliance. Industry foundation classes (IFC). Website, October 2012. http://buildingsmart.com/standards/ifc.

[23] buildingSMART Alliance. International framework for dictionaries (IFD). Website, October 2012. http://buildingsmart.com/standards/ifd.

[24] buildingSMART Alliance. Process - information delivery manual (IDM). Website, October 2012. http://www. buildingsmart.com/standards/idm.

[25] Ciftcioglu, Sariyildiz, and Bittermann. Building performance analysis supported by ga. IEEE Congress on Evolutionary Computation, pages 859- 866, 2007. 
[26] Markus Clermont. Heuristics for the automatic identification of irregularities in spreadsheets. SIGSOFT Softw. Eng. Notes, 30(4):1-6, May 2005.

[27] Comsol. Comsol multiphysics. Website, September 2012. http://www . comsol.com/products/multiphysics/.

[28] Angela Czerwinski1, Thomas H. Kolbe, Lutz Plmer, and Elke Stcker-Meier. Spatial data infrastructure techniques for flexible noise mapping strategies. International Conference on Environmental Informatics - Managing Environmental Knowledge, pages pp.99-106, 2006.

[29] Dassault System. Isight \& the simulia execution engine. Website, September 2012. http://www.3ds.com/products/simulia/portfolio/ isight-simulia-execution-engine/overview/.

[30] S.M. Easterbrook. Climate change: a grand software challenge. In Proceedings of the FSE/SDP workshop on Future of software engineering research, pages 99-104. ACM, 2010.

[31] C. Eastman, P. Teicholz, R. Sacks, and K. Liston. BIM Handbook: a guide to building information modelling for owners, managers, designers, engineers and contractors. John Wiley and Sons, 2011.

[32] Chuck Eastman. The evolution of aec interoperability. EG-ICE, 2012.

[33] ESRI. Arcgis. Website, November 2012. http://www.esri.com/software/ arcgis.

[34] ESTECO. modefrontier. Website, November 2012. http://www.esteco. com/home/mode_frontier/mode_frontier.html.

[35] F. Flager and J. Haymaker. A comparison of multidisciplinary design, analysis and optimization processes in the building construction and aerospace industries. 24th International Conference on Information Technology in Construction. I. Smith. Maribor, Slovenia, pages 625 - 630, 2007.

[36] Forest Flager, Benjamin Welle, Prasun Bansal, Grant Soremekun, and John Haymaker. Multidisciplinary process integration and design optimization of a classroom building. Journal of Information Technology in Construction, 2009.

[37] gbXML Board of Directors. Open green building xml schema. Website, November 2012. http://gbxml.org/.

[38] P. Geyer. Component-oriented decomposition for multidisciplinary design optimization in building design. Advanced Engineering Informatics, 23(1):12 - 31, 2009 . 
[39] Philipp Geyer. Multidisciplinary grammars supporting design optimization of buildings. Research in Engineering Design, 18:197-216, 2008.

[40] GraphML Team. The GraphML file format. Website, June 2007. http:// graphml.graphdrawing.org/.

[41] Grasshopper Team. Grasshopper - generative modeling for rhino. Website, August 2011. http://www.grasshopper3d.com.

[42] Gerhard Grger, Thomas H. Kolbe, Claus Nagel, and Karl-Heinz Hfele. OGC City Geography Markup Language (CityGML) Encoding Standard. Open Geospatial Consortium, April 2012. http://www.opengis.net/spec/citygml/2.0.

[43] F. Hermans, M. Pinzger, and A. Deursen. Supporting professional spreadsheet users by generating leveled dataflow diagrams. 33rd International Conference on Software Engineering (ICSE), pages 451-460, 2011.

[44] F. Hermans, M. Pinzger, and A. Deursen. Detecting and visualizing interworksheet smells in spreadsheets. 34th International Conference on Software Engineering (ICSE), pages 441-451, 2012.

[45] Robina Hetherington, Robin Laney, Stephen Peake, and David Oldham. Integrated building design, information and simulation modelling: the need for a new hierarchy. Building Simulation 2011 Sydney, Australia, 2011.

[46] J. Hietanen and S. Final. IFC model view definition format. International Alliance for Interoperability, 2006.

[47] K. Hodnigg and R. Mittermeir. Metrics-based spreadsheet visualization support for focused maintenance. Proceedings of European Spreadsheet Risks Interest Group (EuSpRIG), pages 79-94, 2008.

[48] Holistic City Software. Pixelactive3d. Website, July 2010. http:// pixelactive3d.com/.

[49] Holistic City Software. Citycad - technology for liveable cities. Website, September 2012. http://www.holisticcity.co.uk/.

[50] Infralution. Infralution virtual treeview C\# component. Website, May 2009. http://infralution.com/virtualtree.html.

[51] Maria Jean and Johnstone Hall. A risk and control-oriented study of the practices of spreadsheet application developers. In In Proceedings of the 29th Hawaii International Conference on System Sciences, pages 364-373, 1996. 
[52] B. Kankuzi and Y. Ayalew. An end-user oriented graph-based visualization for spreadsheets. Proceedings of the 4 th international workshop on End-user software engineering, 2008.

[53] James Keirstead, Nouri Samsatli, and Nilay Shah. Syncity: An integrated tool kit for urban energy systems modelling. Proceedings of the 5th Urban Research Symposium, Marseille, 2009.

[54] H Kepran. Heidelberg. creating a framework for integrated resource management. Technical report, ICLEI, 2002.

[55] Khronos Group Inc. Collaborative design activity (collada) format. Website, July 2010. https://collada.org/mediawiki/index.php/COLLADA_-_ Digital_Asset_and_FX_Exchange_Schema.

[56] Khronos Group Inc. Collada - digital asset and fx exchange schem. Website, July 2010. http://www.khronos.org/.

[57] Khronos Group Inc. Collada version 1.5 release notes. Website, July 2010. http: //www.khronos.org/files/collada_1_5_release_notes.pdf.

[58] Gavin Killip. Transforming the UKs existing housing stock. Technical report, Environmental Change Institute, University of Oxford and Federation of Master Builders, 2008.

[59] T. H. Kolbe and G. Grger. Towards unified 3d city models. ISPRS Comm. IV Workshop "Challenges in Geospatial Analysis, Integration and Visualization II" Stuttgart, Germany, 2003.

[60] Thomas H. Kolbe. CityGML - exchange and storage of virtual 3d city models. Website, November 2012. http://www.citygml.org/.

[61] H. Liang and D. Birch. Extraction and analysis methodology for supporting complex sustainable design. 18th International Conference on Engineering Design (ICED11), 2011.

[62] H. Liang and D. Birch. Supporting complex and sustainable ecocity design using extraction and analysis methodology (EAM). Ecocity World Summit, Montreal, 2011.

[63] P J Littlefair. Site layout planning for daylight and sunlight: a guide to good practice. Building Research Establishment, 1991.

[64] K.J. Lomas, H. Eppel, C.J. Martin, and D.P. Bloomfield. Empirical validation of building energy simulation programs. Energy and Buildings, 26(3):253 - 275, 1997. 
[65] Chris Luebkeman and Alvise Simondetti. Practice 2006: Toolkit 2020. In IanF.C. Smith, editor, Intelligent Computing in Engineering and Architecture, volume 4200 of Lecture Notes in Computer Science, pages 437-454. Springer Berlin Heidelberg, 2006. Video online at http://vimeo.com/1565212.

[66] Martin Manning. Did we design better buildings before computers? Website, December 2012. http://thoughts.arup.com/Post/Details/258.

[67] R. Martin and M. Martin. Agile Principles, Patterns, and Practices in C\#. Prentice Hall, 2006.

[68] MathWorks. Matlab. Website, August 2012. http://www . mathworks.co. uk/products/matlab/.

[69] MathWorks. Simulink - simulation and model-based design. Website, November 2012. http://www. mathworks.co.uk/products/simulink/.

[70] W. Mazairac and J. Beetz. Towards a framework for a domain specific open query language for building information models. EG-ICE, 2012.

[71] D.C. Montgomery. Design and Analysis of Experiments. John Wiley \& Sons, 2004.

[72] L. Moreau. The foundations for provenance on the web. Foundations and Trends in Web Science, 2(2-3):99-241, 2010.

[73] S. Muggleton, D. Lin, and A. Tamaddoni-Nezhad. Mc-toplog: complete multiclause learning guided by a top theory. Inductive Logic Programming, pages $238-254,2012$.

[74] OSGeo Project. What is postgis? Website, November 2012. http://postgis . refractions.net/.

[75] J. Page, N. Grange, and N. Kirkpatrick. The integrated resource management (IRM) model - guidance tool for sustainable urban design. 25th Conference on Passive and Low Energy Architecture, 2008. PLEA08.

[76] Robert Pahle. Decision theatre. Website, November 2009. http://dt.asu. edu.

[77] R. R. Panko. What we know about spreadsheet errors. revised edition may 2008. Journal of End User Computing, pages pp.15-21, 2008.

[78] Raymond R. Panko and Salvatore Aurigemma. Revising the panko-halverson taxonomy of spreadsheet errors. Decis. Support Syst., 49(2):235-244, May 2010. 
[79] Phoenix Integration. Phx centerlink. Website, July 2012. http://www . phoenix-int.com/software/phx-centerlink.php.

[80] Phoenix Integration. Phx modelcenter. Website, July 2012. http://www . phoenix-int.com/software/phx-modelcenter.php.

[81] R.L. Plackett and J.P. Burman. The design of optimum multifactorial experiments. Biometrika, pages pp.305-325, 1946.

[82] Quest3d. Quest3d visualisation engine. Website, September 2012. www . Quest3d.com.

[83] J. Reichwein, G. Rothermel, and M. M. Burnett. Slicing spreadsheets: an integrated methodology for spreadsheet testing and debugging. Proceedings of the 2nd conference on Domain-specific languages 1999, 1999.

[84] Rhinoceros Team. Rhinoceros - 3d modelling software. Website, August 2011. http://www.rhino3d.com/.

[85] Sebastien Ros. Ncalc mathematical expressions evaluator for .NET. Website, June 2011. http://ncalc.codeplex.com/.

[86] T.L. Saaty. How to make a decision: the analytic hierarchy process. European journal of operational research, 48(1):9-26, 1990.

[87] Safe Software. Fme desktop and server. Website, November 2012. www.safe. com.

[88] R.R. Senescu. Design Process Communication Methodology (Thesis). Stanford University, 2011.

[89] Kristina Shea, Andrew Sedgwick, and Giulio Antonuntto. Multicriteria optimization of paneled building envelopes using ant colony optimization. EG-ICE Workshop, 13:627-636, 2006.

[90] H. Shiozawa, K. Okada, and Y. Matsushita. 3d interactive visualization for intercell dependencies of spreadsheets. IEEE Symposium on Information Visualization 1999, 1999.

[91] A. Simondetti, S. Roberts, and D. Birch. BEM for collaborative design inception: Harnessing the power of clients' design intuition. The 2012 International Conference on Modeling, Simulation \& Visualization Methods., 2012.

[92] A. Simondetti, S. Roberts, and D. Birch. A practical perspective on computer tools for sustainable building design. Proceedings of the 2012 International EGICE Workshop on Intelligent Computing, Herrsching, Germany, 2012. 
[93] Alvise Simondetti. BEM for collaborative design inception - Harnessing the power of clients design intuition. Arup Foresight and Innovation, October 2011. http://www.driversofchange.com/wp-content/uploads/ 2012/10/Vision_2_FINAL_oct2012_web.pdf.

[94] Alvise Simondetti. BEM for stakeholder engagement - Driving the adoption of radical change and design innovation. Arup Foresight and Innovation, May 2011. http://www.driversofchange.com/wp-content/uploads/ 2011/06/Future-Tools1.pdf.

[95] Alvise Simondetti. Digital environments for experiential design - Enhancing designers perception. Arup Foresight and Innovation, September 2012. http://www.driversofchange.com/wp-content/uploads/ 2012/10/23-Ver_Vison-3_web.pdf.

[96] Jeffrey Smith, Jessica K. Hodgins, Irving Oppenheim, and Andrew P. Witkin. Creating models of truss structures with optimization. ACM Transactions on Graphics - TOG, 21/3:295-301, 2002.

[97] M. Torgersen. Querying in C\#: how language integrated query(linq) works. In Conference on Object Oriented Programming Systems Languages and Applications: Companion to the 22 nd ACM SIGPLAN conference on Object oriented programming systems and applications companion, volume 21, pages 852-853, 2007.

[98] Andrew Troelsen. Pro C\# 2010 and the .NET 4 Platform (5th Edition). Apress, 2010.

[99] UK Cabinet office. Bim task group website. Website, May 2011. http: / /www . bimtaskgroup.org/.

[100] Unity3d. Unity3d games engine. Website, August 2012. http://www . unity3d.com.

[101] U.S. Department of Energy. Energyplus energy simulation software. Website, September 2012. http://apps1.eere.energy.gov/buildings/ energyplus/.

[102] G. Vanderplaats and F Moses. Automated optimal geometry design of structures. Journal of the Structural Division of the American Society of Civil Engineers, 1977.

[103] Gregory J. Ward. The radiance lighting simulation and rendering system. Computer Graphics (Proceedings of '94 SIGGRAPH conference), 1994. 
[104] M. Weiser. Program slicing. Proceedings of the 5th international conference on Software engineering 1981, 1981.

[105] JH Woo, M. J. Clayton, R. E. Johnson, B. E. Flores, and C. Ellis. Dynamic knowledge map: reusing experts' tacit knowledge in the aec industry. Automation in Construction, 13(2):203 - 207, 2004.

[106] J.J. Yi, H. Vandierendonck, L. Eeckhout, and D.J. Lilja. The exigency of benchmark and compiler drift: designing tomorrow's processors with yesterday's tools. Proceedings of the 20th Annual International Conference on Supercomputing, pages pp.75-86, 2006. 